\title{
The entanglement of thrombus formation: systems biology as a novel key
}

Citation for published version (APA):

de Witt, S. M. (2014). The entanglement of thrombus formation: systems biology as a novel key. [Doctoral Thesis, Maastricht University]. Uitgeverij BOXPress. https://doi.org/10.26481/dis.20141127sw

Document status and date:

Published: 01/01/2014

DOI:

10.26481/dis.20141127sw

Document Version:

Publisher's PDF, also known as Version of record

\section{Please check the document version of this publication:}

- A submitted manuscript is the version of the article upon submission and before peer-review. There can be important differences between the submitted version and the official published version of record.

People interested in the research are advised to contact the author for the final version of the publication, or visit the DOI to the publisher's website.

- The final author version and the galley proof are versions of the publication after peer review.

- The final published version features the final layout of the paper including the volume, issue and page numbers.

Link to publication

\footnotetext{
General rights rights.

- You may freely distribute the URL identifying the publication in the public portal. please follow below link for the End User Agreement:

www.umlib.nl/taverne-license

Take down policy

If you believe that this document breaches copyright please contact us at:

repository@maastrichtuniversity.nl

providing details and we will investigate your claim.
}

Copyright and moral rights for the publications made accessible in the public portal are retained by the authors and/or other copyright owners and it is a condition of accessing publications that users recognise and abide by the legal requirements associated with these

- Users may download and print one copy of any publication from the public portal for the purpose of private study or research.

- You may not further distribute the material or use it for any profit-making activity or commercial gain

If the publication is distributed under the terms of Article $25 \mathrm{fa}$ of the Dutch Copyright Act, indicated by the "Taverne" license above, 


\section{THE ENTANGLEMENT OF THROMBUS FORMATION: \\ SYSTEMS BIOLOGY AS \\ A NOVEL KEY}




\section{THE ENTANGLEMENT OF THROMBUS FORMATION:}

\section{SYSTEMS BIOLOGY AS A NOVEL KEY}

Thesis Universiteit Maastricht

ISBN 978-90-8891-973-2

(C) Susanne Maria de Witt, Maastricht 2014

Printed by Proefschriftmaken.nl || Uitgeverij BOXPress, 's-Hertogenbosch Layout and cover by Southern Graphics design 


\title{
THE ENTANGLEMENT OF THROMBUS FORMATION: \\ SYSTEMS BIOLOGY AS \\ A NOVEL KEY
}

\author{
PROEFSCHRIFT \\ ter verkrijging van de graad van doctor aan de Universiteit van Maastricht, \\ op gezag van de Rector Magnificus, Prof. Dr. L.L.G. Soete, \\ volgens het besluit van het College van Decanen, \\ in het openbaar te verdedigen op \\ donderdag 27 november 2014 om 12.00 uur
}

door

\section{Susanne Maria de Witt}

Geboren op 17 juli 1986 te Brunssum 


\section{Promotor}

Prof. Dr. J.W.M. Heemskerk

\section{Copromotor}

Dr. J.M.E.M Cosemans

\section{Beoordelingscommissie}

Prof. Dr. H. ten Cate (voorzitter)

Prof. Dr. A. Bast

Dr. Ir. Y. Henskens

Dr. B. de Laat

Prof. Dr. S. Watson (Birmingham University, Birmingham, UK)

Financial support by the Dutch Heart Foundation for the publication of this thesis is gratefully acknowledged.

Additional financial support for publication of this thesis by Stichting Hartsvrienden RESCAR is gratefully acknowledged.

Additional support for publication of this thesis by Octapharma, Stichting Fonds voor het Hart, Federatie van Nederlandse Thrombosediensten, Willy van Heumenfonds. 


\section{CONTENTS}

$\begin{array}{lll}\text { Chapter } 1 & \text { General introduction } & 7\end{array}$

Chapter 2 Monitoring in vitro thrombus formation with novel microfluidic devices 19

Chapter 3 Multi-parameter assessment of thrombus formation on microspotted 33 arrays of thrombogenic surfaces

Chapter 4 Identification of platelet function defects by multi-parameter 43 assessment of thrombus formation

Chapter 5 Distinct role of von Willebrand factor triplet bands in glycoprotein Ib- 65 dependent platelet adhesion and thrombus formation under flow

Chapter 6 Supporting roles of platelet thrombospondin-1 and CD36 in thrombus 79 formation on collagen

Chapter $7 \quad$ Insights into platelet-based control of coagulation 91

Chapter 8 Genes in arterial thrombus formation and bleeding: translation of 109 meta-data from mouse studies

$\begin{array}{lll}\text { Chapter } 9 & \text { General discussion } & 129\end{array}$

Summary

Samenvatting

Dankwoord

Publications 



\section{CHAPTER 1}

GENERAL INTRODUCTION 


\section{Platelets in the classical model of thrombus formation}

Platelets play an essential role in the cessation of bleeding at sites of vascular injury (hemostasis) by aggregating into a thrombus to seal off the damaged area $^{1}$. The adhered platelets release a plethora of bioactive substances from their dense and a-granules, which stimulate the healing of the thrombus-containing vessel wall. Platelets furthermore contribute to pathological thrombosis particular in the arterial part of the circulation, where the wall shear rate is high. This occurs via a similar process of thrombus formation, triggered for instance by rupture of damage of an atherosclerotic plaque in the artery ${ }^{2,3}$. This rupture leads to cardiovascular disease, as in myocardial infarction or stroke, which are still major causes of death in the industrialized world ${ }^{4}$.

Based on the assumption that platelet interaction with the extracellular matrix protein collagen is a primary event in arterial thrombosis, several research groups have developed molecular models of collagen-dependent thrombus formation $2,3,5,6$. Support for such models comes from multiple in vivo studies, where arterial thrombosis is experimentally induced in mice, and from parallel in vitro studies where whole blood is flown at arterial wall shear rate over a collagen surface ${ }^{6-9}$.In the classical model of thrombus formation (Figure 1), fibrillar collagen together with von Willebrand factor (VWF), which binds to the collagen, are the primary triggers for platelet adhesion and subsequent activation and thrombus formation ${ }^{1,10}$. The bound VWF traps flowing platelets via the receptor glycoprotein Ib-V-IX and integrin $a_{\mathrm{IIb}} \beta_{3}$, while collagen establishes platelet adhesion via the receptors glycoprotein VI and integrin $a_{2} \beta_{1}$. Both in the mouse and human system, these platelet-adhesive receptors, in an orchestrated interplay, induce activation of nearby flowing platelets so that a thrombus gradually is built up ${ }^{11,12-14}$.

Activation of integrin $a_{\mathrm{IIb}} \beta_{3}$ is essential for platelet-platelet interactions via the bridging protein fibrinogen. Continued activation of the integrin is required for maintenance of these interactions and, hence, for thrombus stability ${ }^{15}{ }^{16}$. Another event in platelet activation is a rise in cytosolic free $\mathrm{Ca}^{2+}$ concentration, which leads to integrin activation, platelet shape change (pseudopod and lamellipod formation) and to secretion of platelet dense and a-granules ${ }^{1,17,18}$. The dense granules release nucleotides such as ADP and ATP, which support thrombus formation by potently stimulating other platelets ${ }^{19,20}$. The effects of released ADP are antagonized by purinergic receptor blockers that are in regular clinical use ${ }^{21}$. The a-granules release a large number of proteins with different roles. These include VWF and fibrinogen, which in an autocrine way can support thrombus formation. However, also other granular proteins may contribute to thrombus formation, but this is unclear. One of the consequences of a-granule release is exposure of the proinflammatory glycoprotein P-selectin (CD62P) at the platelet surface, which enforces platelet-leukocyte interactions ${ }^{2}$. Platelets furthermore produce the secondary mediator thromboxane $\mathrm{A}_{2}$, formed from its precursor arachidonic acid, a process that is irreversibly inhibited by the drug aspirin?. Together, all these autacoid effectors can contribute to the recruitment and capture of passing platelets into a growing thrombus.

Recently, interest has been raised in heterogeneity of platelet responses during thrombus formation ${ }^{22}$. The majority of collagen-adhered platelets assemble into aggregates via activated integrins. These platelets display moderate rises in cytosolic $\mathrm{Ca}^{2+}$, sufficient for shape change, integrin $\mathrm{a}_{\mathrm{IIb}} \beta_{3}$ activation, thromboxane formation and secretion of granule contents ${ }^{18}$. However, a subpopulation of these platelets displays a continued high cytosolic $\mathrm{Ca}^{2+}$ concentration, which triggers membrane blebbing and exposure of phosphatidylserine at their outer membrane via the scramblase protein anoctamin 6 (TMEM16F) ${ }^{23}$. Exposure of phosphatidylserine by collagen-adhered platelets is a known marker of glycoprotein VI activity ${ }^{11,24,25}$. This population of platelets is active in coagulation, in particular by supporting the formation of thrombin and fibrin at their surface ${ }^{26}$. The major mechanism for $\mathrm{Ca}^{2+}$ influx into platelets is that of store-operated $\mathrm{Ca}^{2+}$ entry via the cation channel Orai1 ${ }^{27}$. Mutations in this channel are found in patients with severe combined immunodeficiency syndrome ${ }^{28}$. The consequences of such mutations for thrombus formation have not yet been investigated in detail. 
Thrombin, which is generated at the surface of procoagulant platelets from prothrombin by the coagulation factors Xa and Va stimulates other platelets via the G-coupled protease-activated receptors, PAR1 and PAR429, 30 . Thrombin furthermore cleaves fibrinogen into fibrin monomers and activates several coagulation factors. Hence, thrombin plays a central role at the interface of platelet and coagulation activation ${ }^{31}$. Interestingly, another effect of thrombin secondary to fibrin formation is stimulation of platelet-dependent clot retraction via activated $a_{\mathrm{IIb}} \beta_{3}$. In flow assays a similar process is observed, namely contraction of a thrombus formed on collagen, a process that contributes to stabilization of the thrombus ${ }^{32}, 33$. The regulation of this process is not well understood.

In the present thesis, thrombus formation is defined as the overall result of platelet activation, aggregation and procoagulant activity during whole blood flow over a thrombogenic surface in vitro or in vivo, regardless of the presence or absence of fibrin.

\section{Limitations and extensions of the classical model}

The classical model of thrombus formation concentrates on the primary function of collagen/VWF to induce platelet activation under flow and, thereby, form a platelet aggregate. However, in recent years, it has become clear that this model may need to be extended or adapted, to better understand the roles of: unexplored granule-derived components from platelets, different molecular forms of VWF, and platelet-adhesive substrates other than collagen.

\section{Roles of components in a-granules and dense granules}

Lack of platelet a-granules is associated with a mild to moderate bleeding tendency in patients with congenital gray platelet syndrome. Recently, this disease has been linked to mutations in the NBEAL2 gene, leading to defects in platelet activation properties ${ }^{34-36}$. Another bleeding disease that is characterized by few granules and giant platelets, is the May-Hegglin anomaly, likely caused by mutations in the MYH9 gene ${ }^{37,38}$. Consequences of these secretion defects for the process of thrombus formation are not well investigated. Likewise, it is not fully clear how components stored in the granules are involved in this process. One of the most highly expressed storage proteins in terms of copy numbers is thrombospondin- ${ }^{39}$. Given that it is a key ligand of the platelet receptor CD36, also present at high copy numbers ${ }^{40}$, the question raises if an inability of thrombospondin-CD36 interactions may contribute to the gray platelet syndrome.

The platelet dense granules are packed with non-protein compounds like $\mathrm{Ca}^{2+}$, ADP, ATP, serotonin and polyphosphates ${ }^{7}$. A bleeding diathesis linked to deficiencies in platelet dense granules is presented by patients with Hermansky-Pudlak syndrome ${ }^{37,41}$. At least eight disease-rated genes have been identified (HPS1-HPS8), where mutations lead to a similar phenotype, for instance albinism ${ }^{37}$

\section{Different forms of VWF}

How different types of collagen support thrombus formation has been investigated in considerable detail $^{42,43}$. However, much less is clear of the contribution of different forms of VWF present in the blood plasma and deposited at the vessel wall. The importance of VWF as a bridging molecule, linking platelet adhesion and activation, may appear from the fact that quantitative or qualitative defects in this protein lead to the bleeding disorder, von Willebrand disease ${ }^{44,45}$. The large glycoprotein VWF is synthesized in endothelial cells and megakaryocytes, where it concentrates in granules that - in endothelial cells - are either subjected to continuous secretion or to regulated exocytosis ${ }^{46,47}$. VWF that is synthesized in megakaryocytes assembles in the platelet a-granules. In both endothelial cells and platelets, the VWF is stored as large multimers ranging in size from 500 to $20,000 \mathrm{kDa}$, but in the blood VWF also circulates as smaller multimers ${ }^{48}$.

The metalloproteinase ADAMTS-13, released from endothelial cells, is one of the predominant proteases cleaving large VWF multimers into smaller, less active multimers ${ }^{49}$. Hence, low le- 


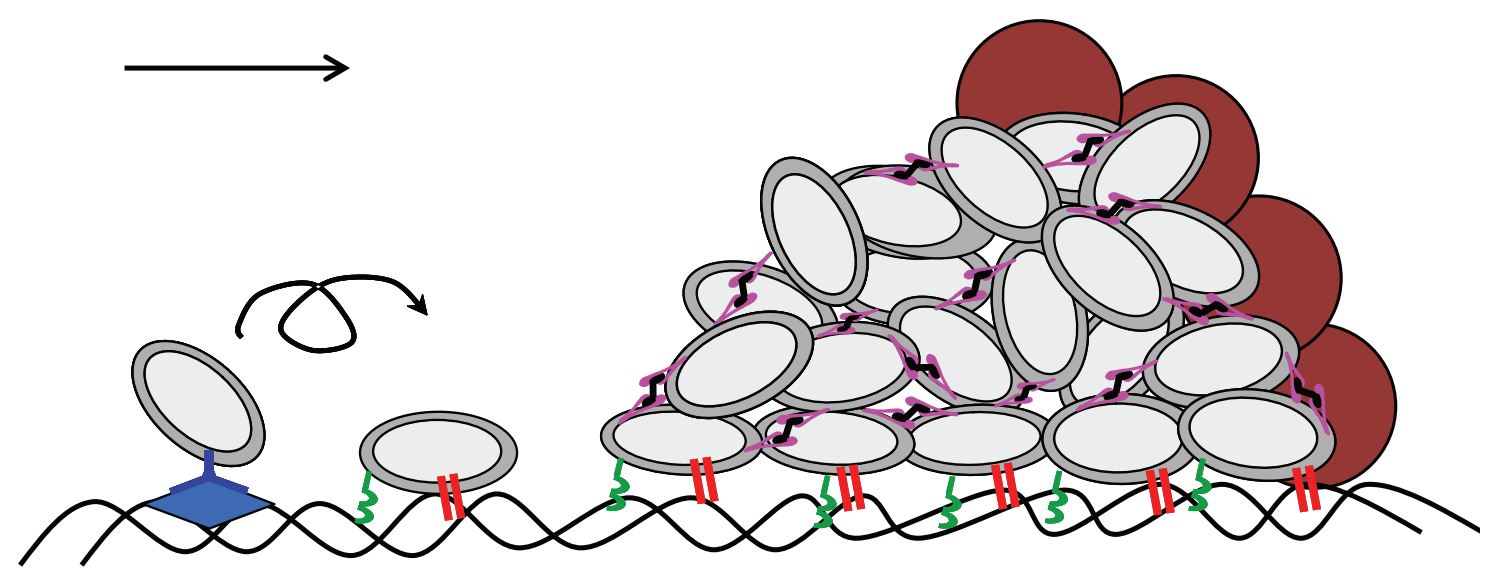

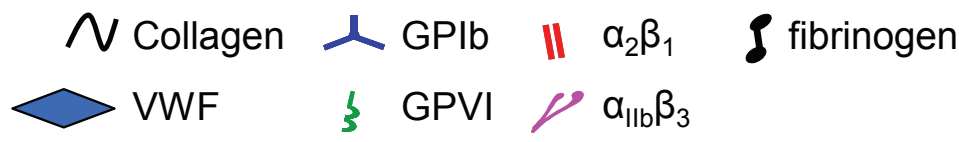

Figure 1. The classical model of collagen-dependent thrombus formation. In this model, collagen that is exposed after vessel wall damage is considered to be the main platelet-activating substance triggering the formation of an arterial thrombus. Exposed collagen fibers bind VWF, to which under high wall-shear conditions platelets (grey) bind via the glycoprotein Ib-V-IX complex (GPIb). Subsequent stable platelet-collagen interaction is achieved via the receptors GPVI and integrin $a_{2} \beta_{1}$, resulting in activation of platelets. Autocrine mediators such as ADP and thromboxane $A_{2}$ activate other, flowing platelets and trap these into the thrombus. GPVI activation also leads to the formation of blebbing, phosphatidylserine-exposing platelets (brown), which provide a surface for the generation of thrombin. Arrow indicates the direction of flow.

vels of ADAMTS-13 associate with increased VWF activity and, thereby, a higher thrombosis tendency ${ }^{50}$. ADAMTS-13 cleaves VWF in the A2 domain, a process that is accelerated by high shear stress, factor VIII, and the platelet glycoprotein Ib-V-IX complex ${ }^{51,52}$. Asymmetric cleavage of the A2 domain by ADAMTS-13, results in multimers with slower, intermediate or faster migrating triplet bands. ${ }^{53,54}$. The smaller and larger triplet bands either lack or contain one $140 \mathrm{kDa} N$-terminal peptide sequence compared to the intermediate VWF triplet band, respectively ${ }^{55}$. Because the $\mathrm{N}$-terminal sequence contains binding sites for factor VIII and glycoprotein Ib, it can be expected that the smaller VWF forms with extra $\mathrm{N}$-terminal sequences are more active in platelet binding. However, this has not been investigated.

\section{Contributions of vascular components other than collagen}

Platelets express adhesive receptors not only for collagen, but also for several other vascular and plasma proteins ${ }^{56}$. Limited flow adhesion studies have been performed on surfaces coated with fibrinogen ${ }^{57}$, fibronectin ${ }^{58}$, vitronectin ${ }^{59}$, 60 , laminin ${ }^{61}$, or thrombospondin- ${ }^{62}$. In all cases thrombus formation could be observed. However, the adhesiveness of these surfaces has not been compared with that of collagens. A relevant receptor on platelets is the C-type lectin-like receptor CLEC-2, which lacks a physiological ligand in the vasculature, but is known to contribute to arterial thrombus formation in vivo as strongly as the collagen receptor, glycoprotein $\mathrm{VI}^{63,64}$. In vitro, CLEC-2 can be stimulated with the snake venom rhodocytin, but this ligand has not been examined in flow adhesion studies.

For the present thesis, we reasoned that direct comparison in flow adhesion experiments of all these platelet-adhesive proteins, alone or in combination, should give better insight into the relative roles of the different platelet adhesion receptors in thrombosis. Other authors have demonstrated that precise measurement of the platelet $\mathrm{Ca}^{2+}$ response can predict the extent of thrombus formation on collagen ${ }^{65}$. We aimed to extend this work by comparing a large variety of adhesive surfaces and platelet activation parameters upon thrombus formation. The hypothesis is that a 
direct comparison of multiple adhesive surfaces and of multiple activation parameters: (i) will give detailed insight into the multi-dynamics of thrombus formation and the most relevant variables of this process; and (ii) will lead to a high-throughput flow chamber assay to assess platelet function, for instance in a clinical setting.

\section{Clinical testing of platelet function}

Platelet activation tests are widely used in the clinic not only to identify platelet-related bleeding disorders, but also to determine the efficacy of antiplatelet medication. As reviewed elsewhere, a whole range of techniques is available for such testing ${ }^{66}$. Here only assays that are relevant for this thesis are mentioned.

Several recently developed assays use whole blood samples or platelet-rich plasma to simultaneously detect platelet and coagulant activity. These include rotational thromboelastography (ROTEM) or thromboelastometry (TEM) and methods of calibrated automated thrombin generation (CAT). The ROTEM or TEM tests determine the kinetics of fibrin clot formation and platelet-dependent clot retraction ${ }^{67-69}$. These tests are sensitive to the presence of platelets and fibrinogen under conditions of coagulation ${ }^{70}$. The CAT assay in its most popular form measures tissue factor-triggered thrombin generation, in a way where the coagulant surface is provided by either activated platelets or phospholipids from other sources ${ }^{71}, 72$. Although these tests can use whole blood samples, their main focus is on coagulation activity rather than on platelet function.

Light transmission aggregometry is regarded as the gold standard of platelet function testing, and is as such frequently employed for the identification of platelet dysfunctions ${ }^{73}$. In cuvettes, samples of platelet-rich plasma or washed platelets are triggered with an agonist such as collagen or ADP, and the changes in light transmission are recorded ${ }^{74}$. Measured are light transmission changes caused by platelet shape change and integrin $a_{\mathrm{IIb}} \beta_{3}$-dependent platelet aggregation $^{75}$. Secretion of ATP from dense granules can be measured by luminescence aggregometry ${ }^{76,77}$. Because it requires centrifugation steps to obtain a platelet preparation, these aggregometry tests are rather time-consuming.

Specific functional assays, using whole blood or platelets in plasma, are often employed to check for the efficacy of antiplatelet medication ${ }^{73,75}$. Regularly employed are the PlateletWorks, VerifyNow and VASP tests, which will not be discussed further. The PFA-100 system is the only validated test to assess platelet activation under high shear flow conditions. It measures capillary occlusion due to platelet aggregation onto a grid coated with collagen. However, its use is mostly limited to the detection of acquired or congenital von Willebrand disease (VWD) or $\mathrm{a}_{\mathrm{IIb}} \beta_{3}$ deficiencies $^{73}$.

Several laboratories have been working on the development of platelet function tests for high-throughput screening purposes. Recently, the optical multichannel (Optimul) assay has been proposed as an easy-to-handle and cheap alternative for light transmission aggregometry ${ }^{78,79}$. It uses a 96-well plate, containing lyophilized platelet agonists, and detects the changes in light absorbance upon aggregation of platelets in plasma. Other methods, not requiring centrifugation steps, use 96-well plates in combination with (diluted) whole blood samples. Platelet activation in response to the agonists is tested here with fluorescent-labeled antibodies against surface activation markers (activated integrins, P-selectin) and automated flow cytometry after fixation ${ }^{80,81 .}$

A drawback of several of the tests mentioned above is that they are applicable for a limited number of disease states ${ }^{66}$. Thereby, they are not always critically validated in terms of checking for variation due to technical and pre-analytical factors, and for intra- and inter-subject factors ${ }^{82}$.

For more than a decade, it has been argued that parallel-plate flow chambers can help in the clinically testing of platelet function, as they mimic the in vivo situation of blood flow ${ }^{83}$. Flow chamber based assays of thrombus formation measure platelet activation and aggregation as well as platelet procoagulant activity at the same time, and can use different platelet-adhesive surfaces such as collagen, endothelial cells or atherosclerotic plaque material${ }^{42,84,85}$. However, the flow 
chamber technology lacks standardization in chamber construction, operating procedures and data analysis, which limitations unfortunately are not overcome by the use of small-size microfluidic flow devices ${ }^{86}$. With all the knowledge and expertise present at the Heemskerk/Cosemans lab, one of my intentions was to further develop and standardize this technology to a rapid multi-parameter and high-throughput test that can make its way to the hospital.

\section{Systems biology and thrombus formation}

Adequate handling of "big data" becomes more and more important in medical biology. High-throughput technologies, for instance in genomics and proteomics, generate enormous amounts of data that require novel scientific approaches for capturing, handling, curating, systematic analysis and visualization ${ }^{87}$. Challenges are provided by not only the large size, but also the high complexity of new data arrays. Systems biology (systeomics) is a novel interdisciplinary field to study complex interactions in biological systems, using a holistic approach ${ }^{87,88,89}$. Originally, the system of omics studies was a given cell or organism. Systems biology techniques aimed to increase the ability: (i) to understand all components in the cell or organism up to the molecular level, (ii) to predict the future status of the cell or organism under normal conditions, (iii) to forecast responses to a given input stimulus, and (iv) to estimate the changes in system behaviour upon perturbation of one or more of the components ${ }^{89}$. However, in recent days, systems biology approaches are increasingly used to understand the component and molecular interactions in other types of biological systems.

In platelet research, systems biology tools have been used only limitedly so far, for example by the Diamond group. ${ }^{65}$ Analysis of large-scale data of platelet proteomics has given insight into the quantitative molecular composition of over 4000 platelet proteins ${ }^{39}$, platelet mRNA species ${ }^{36}$ and protein fragments secreted from platelets ${ }^{90}$. Such data appear to be crucial for increased understanding of the role of platelets in health and disease, as well as for identifying potentially new biomarkers or drug targets ${ }^{91}$. In the interpretation of these omics data, peer reviewed and curated functional protein databases such as the PlateletWeb ${ }^{92}$ and the Reactome ${ }^{93}$ have appeared to be highly valuable. While the PlateletWeb contains information from human platelet studies only, the Reactome is cross-referenced with other bioinformatics databases. In this context, the Gene Ontology Consortium database is mentioned, aiming to integrate data on the functions of gene products across a range of organisms ${ }^{94}$. Most of these databases are accessible via open online sources, which provide support in network visualization, interpretation, analysis and clustering of complex pathways.

In small size scientific studies, the curating and analysis of data is usually performed in a supervised way. However, in case of high-throughput studies, analysis can be less straightforward so that methods have been developed to handle the more complex datasets. A usual first step is scaling, for instance by normalizing multivariate data on a fixed scoring scale. This is followed by the construction of algorithms aimed to understand the normalized data, for instance by dimension reduction, classification or regression analysis ${ }^{89}$. Cluster analysis of data can be performed in a supervised or an unsupervised (hypothesis-free) hierarchical way ${ }^{95}$.

Network analysis of genomic or proteomic data can be done with programs like Cytoscape. This tool allows visualization of the interactions between the proteins or genes of interest, which can be obtained from the IntAct database. In the network, the proteins or genes are represented in

so-called nodes and the interactions are symbolized by edges ${ }^{96}$. Furthermore, new datasets can be combined with already available datasets. These systems biology techniques are emerging in the field of thrombosis and hemostasis, and may be of great value in the high-throughput approaches described in this thesis.

\section{Thrombus formation and translational research}

In the fight against cardiovascular disease, there is a continued search for new targets for antiplatelet and anticoagulant drugs that reduce thrombosis but not increase the bleeding risk. In 
order to understand the contribution of platelet and coagulation proteins in thrombosis, genetically modified mice deficient in specific genes have been generated, and used for in vivo determination of arterial thrombosis and tail bleeding time. Current understanding is that such experimental thrombosis studies are relevant for the human situation, although it should be realized that there are significant differences at the molecular and structural level with varying degrees of homology between mouse and human proteins. So far, more than thousand mouse studies with more than hundred knockouts have been carried out, providing insight into key molecular processes of murine thrombosis and hemostasis. However, reviews attempting to relate these mouse studies are mostly limited to qualitative descriptions of the effects of genetic modification, which makes quantitative comparison a difficult task ${ }^{97-99}$. One important reason for this is the great variation in methods and procedures employed for the in vivo measurements, thus pointing for a need of future standardization of these methods ${ }^{100}$. On the other hand, in vitro flow chamber studies performed in the same mice often give similar results as in vivo studies of arterial thrombus formation ${ }^{83}$. We hypothesized that, in spite of the methodological variation, systems biology approaches can be helpful in performing a systematic meta-analysis combining the results of the majority of in vivo and in vitro mouse studies.

\section{Aims and outline of this thesis}

In this thesis, the process of thrombus formation is studied in a translational and preclinical perspective by means of a systems biology approach. Following chapter $\mathbf{1}$ as a general introduction, chapter 2 provides an overview of the currently available microfluidic devices for applications in the field of thrombosis and hemostasis. Sketched are possibilities for use of such microfluidic devices in the preclinical testing of high-throughput analysis of platelet function in the environment of flowing whole-blood on a variety of platelet-adhesive substrates.

Chapter 3 describes standard protocols for multi-parameter measurement of the roles of platelets in thrombus formation, as assessed with various fluorescence microscopic systems. Detailed information is provided on the multispot technique, the blood drawing, the flow perfusion protocol, the microscopy, and the methods of image analysis. The aim of this chapter is to provide guidance through all steps in the preparation and performance of a successful whole-blood perfusion experiment. In the subsequent experimental chapter 4, a large number of platelet-adhesive surfaces is compared for their efficacy to support platelet adhesion and thrombus formation at various wall shear rates. This work is aimed at assessing the relative roles in these processes of the major adhesive receptors, glycoproteins Ib, and VI, CLEC-2, CD36, and the integrins $a_{\mathrm{IIb}} \beta_{3}, a_{v} \beta_{3}$, $a_{2} \beta_{1}, a_{5} \beta_{1}$ and $a_{6} \beta_{1}$. Also compared are the various platelet responses during thrombus formation, i.e. integrin activation, granule secretion and phosphatidylserine exposure. Another aim of this systematic analysis is to determine which surfaces/receptors and activation parameters are most suited for a screening assay to determine platelet function deficiencies in patients with bleeding disorders.

In chapter 5, the role of VWF multimers in supporting thrombus formation is re-examined in greater detail. Therefore, plasma-derived VWF was separated into fractions with similar multimeric composition, but different triplet band patterns. Studied was the ability of these fractions to bind to glycoprotein Ib, and to support glycoprotein Ib-dependent platelet adhesion. Chapter $\mathbf{6}$ focusses on the role of thrombospondin-1, as a major component released from the platelet a-granules, in the support of thrombus formation on a collagen surface. Since earlier studies had identified the glycoprotein CD36 as a major platelet receptor for thrombospondin-1, the role of CD36 in thrombospondin-1-dependent platelet adhesion and thrombus formation was examined as well. Mice deficient in either thrombospondin-1 or CD36 were used for these studies, carried out in vivo and in vitro.

During the process of thrombus formation, platelets interact with the coagulation system in several ways, i.e. by exposure of procoagulant phosphatidylserine, by supporting thrombin and 
fibrin generation, and by regulating the contraction of a fibrin clot. In chapter 7, an inventory based on the published literature is made, in order to categorize which genetic deficiencies (in mouse) and which pharmacological interventions (in man) result in alterations in these different procoagulant roles of platelets. Particular attention was paid to the modulation - by genetic modification or pharmaca - of these roles via changes in platelet $\mathrm{Ca}^{2+}$ signaling and homotypic platelet-platelet interactions. Chapter 8 presents a meta-analysis, also based on the literature, in which a quantitative scoring scale was developed to systematically examine the effects of genetic deficiency in mouse on experimental arterial thrombosis in vivo as well as on thrombus formation with flow chambers in vitro. In addition, a comparison was made with the effect of genetic deficiency on tail bleeding time. Online available systems biology tools were used to translate this 'scoring of mouse genes' to the human situation, for instance by integrating the mouse data into networks of human genes implicated in platelet function. This systematic approach may lead to new possible targets for antithrombotic therapies. Finally, in chapter $\mathbf{9}$ the the principle findings of this thesis are discussed in a broader perspective.

\section{References}

1. Versteeg HH, Heemskerk JW, Levi M, Reitsma PH. New fundamentals in hemostasis. Physiol Rev 93, 327-358 (2013).

2. Ruggeri ZM. Platelets in atherothrombosis. Nat Med 8, 1227-1234 (2002).

3. Jackson SP. Arterial thrombosis: insidious, unpredictable and deadly. Nat Med 17, 1423-1436 (2011).

4. World Health Organization. Factsheet Cardiovascular diseases Factsheet No: 317, http://www.who.int/ mediacentre/factsheets/fs317/en/ (2013).

5. Gibbins JM. Platelet adhesion signalling and the regulation of thrombus formation. J Cell Sci 117, 3415-3425 (2004).

6. Nieswandt B, Watson SP. Platelet-collagen interaction: is GPVI the central receptor? Blood 102, 449461 (2003).

7. Swieringa F, Kuijpers MJ, Heemskerk JW, van der Meijden PE. Targeting platelet receptor function in thrombus formation: the risk of bleeding. Blood Rev 28, 9-21 (2014).

8. Watson SP, Herbert JMJ, Pollitt AY. GPVI and CLEC-2 in hemostasis and vascular integrity. J Thromb Haemost 8, 1457-1467 (2010).

9. Stegner D, Nieswandt B. Platelet receptor signaling in thrombus formation. J Mol Med 8, 109-121 (2011).

10. Cosemans JM, Angelillo-Scherrer A, Mattheij NJ, Heemskerk JW. The effects of arterial flow on platelet activation, thrombus growth, and stabilization. Cardiovasc Res 99, 342-352 (2013).

11. Siljander PR, Munnix IC, Smethurst PA, Deckmyn H, Lindhout T, et al. Platelet receptor interplay regulates collagen-induced thrombus formation in flowing human blood. Blood 103, 1333-1341 (2004).

12. Lecut C, Feijge MA, Cosemans JM, Jandrot-Perrus M, Heemskerk JW. Fibrillar type I collagens enhance platelet-dependent thrombin generation via glycoprotein VI with direct support of $a_{2} \beta_{1}$ but not $a_{\mathrm{IIb}} \beta_{3}$ integrin. Thromb Haemost 94, 107-114 (2005).

13. Nieswandt B, Brakebusch C, Bergmeier W, Schulte V, Bouvard D, Mohtari-Nejad R, Lindhout T, Heemskerk JW, Zirngibl $H$, Fässler R. Glycoprotein VI but not $a_{2} \beta_{1}$ integrin is essential for platelet interaction with collagen. EMBO J 20, 2120-2130 (2001).

14. Auger JM, Kuijpers MJ, Senis YA, Watson SP, Heemskerk JW. Adhesion of human and mouse platelets to collagen under shear: a unifying model. FASEB J 19, 825-827 (2005).

15. Cosemans JM, Munnix IC, Wetzker R, Heller R, Jackson SP, Heemskerk JW. Continuous signaling via PI3K isoforms $\beta$ and $y$ is required for platelet ADP receptor function in dynamic thrombus stabilization. Blood 108, 3045-3052 (2006).

16. Cosemans JM, Iserbyt BF, Deckmyn $\mathrm{H}$, Heemskerk JW. Multiple pathways to switch platelet integrins on and off. J Thromb Haemost 6, 1253-1261 (2008).

17. Heemskerk JW, Sage SO. Calcium signalling in platelets and other cells. Platelets 5, 295-316 (1994).

18. Heemskerk JW, Bevers EM, Lindhout T. Platelet activation and blood coagulation. Thromb Haemost 88, 186-193 (2002).

19. Gachet C. P2Y ${ }_{12}$ receptors in platelets and other hematopoietic and non-hematopoietic cells. Purin Sign 8, 609-619 (2012).

20. Jones S, Evans RJ, Mahaut-Smith MP. Calcium influx through P2X $X_{1}$ receptors amplifies P2Y receptor-evoked $\mathrm{Ca}^{2+}$ signalling and ADP-evoked platelet aggregation. Mol Pharmacol, in press (2014). 
21. Cattaneo $\mathrm{M}$. The platelet $\mathrm{P} 2 \mathrm{Y}_{12}$ receptor for adenosine diphosphate: congenital and drug-induced defects. Blood 117, 2102-2112 (2011).

22. Munnix IC, Kuijpers MJ, Auger J, Thomassen CM, Panizzi P, van Zandvoort MA, Rosing J, Bock PE, Watson SP, Heemskerk JW. Segregation of platelet aggregatory and procoagulant microdomains in thrombus formation: regulation by transient integrin activation. Arterioscler Thromb Vasc Biol 27, 2484-2490 (2007).

23. Van Kruchten R, Mattheij NJ, Saunders C, Feijge MA, Swieringa F, Wolfs JL, Collins PW, Heemskerk JW, Bevers EM. Both TMEM16F-dependent and TMEM16F-independent pathways contribute to phosphatidylserine exposure in platelet apoptosis and platelet activation. Blood 121, 1850-1857 (2013).

24. Munnix IC, Gilio K, Siljander PR, Raynal N, Feijge MA, Hackeng TM, Deckmyn H, Smethurst PA, Farndale RW, Heemskerk JW. Collagen-mimetic peptides mediate flow-dependent thrombus formation by high- or low-affinity binding of integrin $a_{2} \beta_{1}$ and glycoprotein VI. J Thromb Haemost 6, 2132-2142 (2008).

25. Kuijpers MJ,Schulte V, Bergmeier W, Lindhout T, Brakebusch C, Offermanns S, Fässler R, Heemskerk $J W$, Nieswandt B. Complementary roles of glycoprotein VI and $a_{2} \beta_{1}$ integrin in collagen-induced thrombus formation in flowing whole blood ex vivo. FASEB J 17, 685-687 (2003).

26. Heemskerk JW, Mattheij N, Cosemans JM. Platelet-based coagulation: different populations, different functions. J Thromb Haemost 11, 2-11 (2013).

27. Varga-Szabo D, Braun A, Nieswandt B. STIM and Orai in platelet function. Cell Calcium 50, 270-278 (2011).

28. Feske S, Gwack Y, Prakriya M, Srikanth S, Puppel S, Tanasa B, Hogan PG, Lewis RS, Daly M, Rao A. A mutation in Orai1 causes immune deficiency by abrogating CRAC channel function. Nature 441, 179185 (2006).

29. Heemskerk JW, Feijge MA, Henneman L, Rosing J, Hemker HC. The Ca ${ }^{2+}$-mobilizing potency of a-thrombin and thrombin-receptor-activating peptide on human platelets. Eur J Biochem 249, 547555 (1997).

30. Kahn ML, Nakanishi-Matsui M, Shapiro MJ, Ishihara H, Coughlin SR. Protease-activated receptors 1 and 4 mediate activation of human platelets by thrombin. J Clin Invest 103, 879-887 (1999).

31. Spronk HM, Borissoff JI, ten Cate H. New insights into modulation of thrombin formation. Curr Atheroscler Rep 15, 363 (2013).

32. Auger JM, Watson SP. Dynamic tyrosine kinase-regulated signaling and actin polymerisation mediate aggregate stability under shear. Arterioscler Thromb Vasc Biol 28, 1499-1504 (2008).

33. Ono A, Westein E, Hsiao S, Nesbitt WS, Hamilton JR, Schoenwaelder SM, Jackson SP. Identification of a fibrin-independent platelet contractile mechanism regulating primary hemostasis and thrombus growth. Blood 112, 90-99 (2008).

34. Albers CA, Cvejic A, Favier R, Bouwmans EE, Alessi MC, Bertone P, Jordan G, Kettleborough RN, Kiddle G, Kostadima M, Read RJ, Sipos B, Sivapalaratnam S, Smethurst PA, Stephens J, Voss K, Nurden A, Rendon A, Nurden P, Ouwehand WH. Exome sequencing identifies NBEAL2 as the causative gene for gray platelet syndrome. Nat Gen 43, 735-737 (2011).

35. Gunay-Aygun M, Falik-Zaccai TC, Vilboux T, Zivony-Elboum Y, Gumruk F, Cetin M, Khayat M, Boerkoel CF, Kfir N, Huang Y, Maynard D, Dorward H, Berger K, Kleta, R, Anikster Y, Arat M, Freiberg AS, Kehrel BE, Jurk K, Cruz P, Mullikin JC, White JG, Huizing M, Gahl WA. NBEAL2 is mutated in gray platelet syndrome and is required for biogenesis of platelet a-granules. Nat Gen 43, $732-734$ (2011).

36. Kahr WH, Hinckley J, Li L, Schwertz H, Christensen H, Rowley JW, Pluthero FG, Urban D, Fabbro S, Nixon B, Gadzinski R, Storck M, Wang K, Ryu GY, Jobe SM, Schutte BC, Moseley J, Loughran NB, Parkinson J, Weyrich AS, Di Paola J. Mutations in NBEAL2, encoding a BEACH protein, cause gray platelet syndrome. Nat Gen 43, 738-740 (2011).

37. Nurden AT, Freson K, Seligsohn U. Inherited platelet disorders. Haemophilia 18 Suppl 4, 154-160 (2012).

38. Balduini CL, Savoia A. Genetics of familial forms of thrombocytopenia. Hum Gen 131, 1821-1832 (2012).

39. Burkhart JM, Vaudel M, Gambaryan S, Radau S, Walter U, et al. The first comprehensive and quantitative analysis of human platelet protein composition allows the comparative analysis of structural and functional pathways. Blood 120, e73-82 (2012).

40. Nergiz-Unal R, Rademakers T, Cosemans JM, Heemskerk JW. CD36 as a multiple-ligand signaling receptor in atherothrombosis. Cardiovasc Hematol Agents Med Chem 9, 42-55 (2011).

41. Gunay-Aygun M, Zivony-Elboum, Gumruk F, Geiger D, Cetin M, Khayat M, Kleta R, Kfir N, Anikster Y, Chezar J, Arcos-Burgos M, Shalata A, Stanescu H, Manaster J, Arat M, Edwards H, Freiberg AS, Hart 
PS, Riney LC, Patzel K, Tanpaiboon P, Markello T, Huizing M, Maric I, Horne M, Kehrel BE, Jurk K, Hansen NF, Hansen NF, Cherukuri PF, Jones M, Cruz P, Mullikin JC, Nurden A, White JG, Gahl WA, Falik-Zaccai T. Gray platelet syndrome: natural history of a large patient cohort and locus assignment to chromosome 3p. Blood 116, 4990-5001 (2010).

42. Heemskerk JW, Sakariassen KS, Zwaginga JJ, Brass LF, Jackson SP, Farndale RW. Collagen surfaces to measure thrombus formation under flow: possibilities for standardization. J Thromb Haemost 9, 856858 (2011).

43. Jung SM, Moroi M, Soeijma K, Nakagaki T, Miura Y, Berndt MC, Gardiner EE, Howes JM, Pugh N, Bihan D, Watson SP, Farndale RW. Constitutive dimerization of glycoprotein VI (GPVI) in resting platelets is essential for binding to collagen and activation in flowing blood. J Biol Chem 287, 30000-30013 (2012).

44. Lillicrap D. Von Willebrand disease: phenotype versus genotype, deficiency versus disease. Thromb Res 120, S11-S16 (2007).

45. Sadler JE, Rodeghiero F, ISTH SSC Subcommittee VWF. Provisional criteria for the diagnosis of VWD type 1. J Thromb Haemost 3, 775-777 (2005).

46. Wang JW, Groeneveld DJ, Cosemans G, Dirven RJ, Valentijn KM, Voorberg J, Reitsma PH, Eikenboom J. Biogenesis of Weibel-Palade bodies in von Willebrand's disease variants with impaired von Willebrand factor intrachain or interchain disulfide bond formation. Haematol 97, 859-866 (2012).

47. Springer TA. Von Willebrand factor, Jedi knight of the bloodstream. Blood, in press (2014).

48. Nowak AA, McKinnon TAJ, Hughes JM, Chion ACK, Laffan MA. The O-linked glycans of human von Willebrand factor modulate its interaction with ADAMTS-13. J Thromb Haemost 12, 54-61 (2014).

49. Sonneveld MA, de Maat MP, Leebeek FW. Von Willebrand factor and ADAMTS-13 in arterial thrombosis: a systematic review and meta-analysis. Blood Rev 28, 167-178 (2014).

50. Stockschlaeder M, Schneppenheim R, Budde U. Update on von Willebrand factor multimers: focus on high-molecular-weight multimers and their role in hemostasis. Blood Coagul Fibrinolysis 25, 206-216 (2014).

51. Shim K, Anderson PJ, Tuley EA, Wiswall E, Sadler JE. Platelet-VWF complexes are preferred substrates of ADAMTS-13 under fluid shear stress. Blood 111, 651-657 (2008).

52. Zhang X, Halvorsen K, Zhang CZ, Wong WP, Springer TA. Mechanoenzymatic cleavage of the ultralarge vascular protein von Willebrand factor. Science 324, 1330-1334 (2009).

53. Dent JA, Galbusera M, Ruggeri ZM. Heterogeneity of plasma von Willebrand factor multimers resulting from proteolysis of the constituent subunit. J Clin Invest 88, 774-782 (1991).

54. Furlan M, Robles R, Affolter D, Meyer D, Baillod P, Lammle B. Triplet structure of von Willebrand factor reflects proteolytic degradation of high molecular weight multimers. Proc Natl Acad Sci 90, 75037507 (1993).

55. Fischer BE, Thomas KB, Schlokat U, Dorner F. Triplet structure of human von Willebrand factor. Biochem J 331, 483-488 (1998).

56. Ruggeri ZM, Mendolicchio GL. Adhesion mechanisms in platelet function. Circ Res 100, 1673-1685 (2007)

57. Endenburg SC, Lindeboom-Blokzijl L, Zwaginga JJ, Sixma JJ, de Groot PG. Plasma fibrinogen inhibits platelets adhesion in flowing blood to immobilized fibrinogen. Arterioscler Thromb Vasc Biol 16, 633638 (1996).

58. Chada D, Mather T, Nollert MU. The synergy site of fibronectin is required for strong interaction with the platelet integrin $\mathrm{a}_{\mathrm{IID}} \beta_{3}$. Ann Biomed Eng 34, 1542-1552 (2006).

59. Asch E, Podack E. Vitronectin binds to activated human platelets and plays a role in platelet aggregation. J Clin Invest 85, 1372-1378 (1990).

60. Wu YP, Hasan MA, Chen JY. Fibrin-incorporated vitronectin is involved in platelet adhesion and throm bus formation through homotypic interactions with platelet-associated vitronectin. Blood 104, 10341041 (2004).

61. Inoue O, Suzuki-Inoue K, McCarty OJ, Moroi M, Ruggeri ZM, Kunicki TJ, Ozaki Y, Watson SP. Laminin stimulates spreading of platelets through integrin $a_{6} \beta_{1}$-dependent activation of GPVI. Blood 107, 1405-1412 (2006).

62. Jurk K, Clemetson KJ, de Groot PG, Brodde M, Steiner M, Savion N, Varon D, Sixma JJ, Van Aken H, Kehrel BE. Thrombospondin-1 mediates platelet adhesion at high shear via glycoprotein Ib (GPIb): an alternative/backup mechanism to von Willebrand factor. FASEB J 17, 1490-1492 (2003).

63. May F, Hagedorn I, Pleines I, Bender M, Vogtle T, Eble J, Elvers M, Nieswandt B. CLEC-2 is an essential platelet-activating receptor in hemostasis and thrombosis. Blood 114, 3464-3472 (2009).

64. Suzuki-Inoue K. CLEC-2, the novel platelet activation receptor and its internal ligand, podoplanin. 
Japan J Clin Hematol 50, 389-398 (2009).

65. Flamm MH, Colace TV, Chatterjee MS, Jing H, Zhou S, Jaeger D, Brass LF, Sinno T, Diamond SL. Multiscale prediction of patient-specific platelet function under flow. Blood 120, 190-198 (2012).

66. Michelson AD. Platelet function testing in cardiovascular diseases. Circulation 110, e489-493 (2004).

67. Schols SE, Heemskerk JW, van Pampus EC. Correction of coagulation in dilutional coagulopathy: use of kinetic and capacitative coagulation assays to improve hemostasis. Transfus Med Rev 24, 44-52 (2010).

68. Whiting D, DiNardo JA. TEG and ROTEM: technology and clinical applications. Am J Hematol 89, 228232 (2014).

69. Olde Engberink RH, Kuiper GJ, Wetzels RJ, Nelemans, Lance PJ, Beckers EA, Henskens YM. Rapid and correct prediction of thrombocytopenia and hypofibrinogenemia with rotational thromboelastometry in cardiac surgery. J Cardiothor Vasc Anesth 28, 210-216 (2014).

70. Ninivaggi M, Feijge MAH, Kuiper GJA, Baaten C, Marcus MAE, ten Cate H, Lance MD, Heemskerk JW, Van der Meijden PE. Principal roles of platelets and fibrinogen in whole-blood fibrin clot formation in dilutional coagulopathy determined by thromboelastometry. Thromb Haemost 111, 447-457 (2014).

71. Hemker HC, Giesen P, Al Diera R, Regnault V, Smedt E, Wagenvoord R, Lecompte T, Béguin S. Calibrated automated thrombin generation measurement in clotting plasma. Pathophysiol Haemost Thromb 33, 4-15 (2003).

72. Ninivaggi M, Apitz-Castro R, Dargaud Y, de Laat B, Hemker HC, Lindhout T. Whole-blood thrombin generation monitored with a calibrated automated thrombogram-based assay. Clin Chem 58, 12521259 (2012).

73. Harrison P, Mackie I, Mumford A, Briggs C, Liesner R, Winter M, Machin S. Guidelines for the laboratory investigation of heritable disorders of platelet function. Br J Haematol 155, 30-44 (2011).

74. Cattaneo M, Cerletti C, Harrison P, Hayward CP, Kenny D, Nugent D, Nurden P, Rao AK, Schmaier AH, Watson SP, Lussana F, Pugliano MT, Michelson AD. Recommendations for the standardization of light transmission aggregometry: a consensus of the working party from the Platelet Physiology Subcommittee of SSC/ISTH. J Thromb Haemost (2013).

75. Michelson AD. Methods for the measurement of platelet function. Am J Cardiol 103, 20A-26A (2009).

76. Pai M, Wang G, Moffat KA, Liu Y, Seecharan J, Webert K, Heddle N, Hayward C. Diagnostic usefulness of a lumi-aggregometer adenosine triphosphate release assay for the assessment of platelet function disorders. Am J Clin Pathol 136, 350-358 (2011).

77. Feinman RD, Lubowsky J, Charo I, Zabinski MP. The lumi-aggregometer: a new instrument for simultaneous measurement of secretion and aggregation by platelets. J Lab Clin Med 90, 125-129 (1977).

78. Chan MV, Warner TD. Standardised optical multichannel (optimul) platelet aggregometry using highspeed shaking and fixed time point readings. Platelets 23, 404-408 (2012).

79. Lordkipanidze $\mathrm{M}$, et al. Characterization of multiple platelet activation pathways in patients with bleeding as a high-throughput screening option: use of 96-well Optimul assay. Blood 123, e11-22 (2014).

80. Middelburg RA, Roest M, Ham J, Coccoris M, Zwaginga JJ, van der Meer PF. Flow cytometric assessment of agonist-induced P-selectin expression as a measure of platelet quality in stored platelet oncentrates. Transfusion 53, 1780-1787 (2013).

81. Dovlatova N, Lordkipanidzé M, Lowe GC, Dawood B, May J, Heptinstall S, Watson SP, Fox SC. Evaluation of a whole blood remote platelet function test for the diagnosis of mild bleeding disorders. J Thromb Haemost 12, 660-665 (2014).

82. De Lange NM, Lancé MD, de Groot R, Beckers EA, Henskens YM, Scheepers HC. Obstetric hemorrhage and coagulation: an update. Thromboelastography, thromboelastometry, and conventional coagulation tests in the diagnosis and prediction of postpartum hemorrhage. Obst Gynecol Surv 67, 426-435 (2012).

83. Zwaginga JJ, Sakariassen KS, Nash G, King MR, Heemskerk JW, Frojmovic M, Hoylaerts MF. Flowbased assays for global assessment of hemostasis. Part 2: current methods and considerations for the future. J Thromb Haemost 4, 2716-2717 (2006).

84. Cosemans JM, Kuijpers MJ, Lecut C, Loubele ST, Heeneman S, Jandrot-Perrus M, Heemskerk JW. Contribution of platelet glycoprotein VI to the thrombogenic effect of collagens in fibrous atherosclerotic lesions. Atherosclerosis 181, 19-27 (2005).

85. Westein E, van der Meer AD, Kuijpers MJ, Frimat JP, van den Berg A, Heemskerk JW. Atherosclerotic geometries exacerbate pathological thrombus formation poststenosis in a von Willebrand factor-dependent manner. Proc Natl Acad Sci USA 110, 1357-1362 (2013).

86. Roest M, Reininger A, Zwaginga JJ, King MR, Heemskerk JW. Flow chamber-based assays to measure thrombus formation in vitro: requirements for standardization. J Thromb Haemost 9, 2322-2324(2011). 
87. Bell G, Hey T, Szalay A. Computer science: beyond the data deluge. Science 323, 1297-1298 (2009).

88. Callebaut W. Scientific perspectivism: a philosopher of science's response to the challenge of big data biology. Stud Hist Philos Biol Biomed Sci 43, 69-80 (2012).

89. Altaf-Ul-Amin M, Afendi FM, Kiboi SK, Kanaya S. Systems biology in the context of big data and networks. Biomed Res Internat/ 2014, 428570 (2014).

90. Fong KP, Barry C, Tran AN, Traxler EA, Wannemacher KM, Tang HY, Speichler KD, Blair IA, Specher DW, Grosser T, Brass LF. Deciphering the human platelet sheddome. Blood 117, e15-e26 (2011).

91. Burkhart JM, Gambaryan S, Watson SP, Jurk K, Walter U, Sickmann A, Heemskerk JW, Zahedi RP. What can proteomics tell us about platelets. Circ Res 114, 1204-1219 (2014).

92. Boyanova D, Nilla S, Birschmann I, Dandekar T, Dittrich M. PlateletWeb: a systems biologic analysis of signaling networks in human platelets. Blood 119, e22-34 (2012).

93. Croft D. Building models using Reactome pathways as templates. Meth Mol Biol 1021, 273-283 (2013).

94. Huntley RP, Harris MA, Alam-Faruque $\mathrm{Y}$, Blake JA, Carbon S, Dietze H, Dimmer EC, Foulger RE, Hill DP, Khodiyar VK, Lock A, Lomax J, Lovering RC, Mutowo-Meullenet P, Sawford T, Van Auken K, Wood V, Mungall $\mathrm{CJ}$. A method for increasing expressivity of Gene Ontology annotations using a compositional approach. BMC Bioinformatics 15, 155 (2014).

95. Liquet B, Le Cao KA, Hocini H, Thiebaut R. A novel approach for biomarker selection and the integration of repeated measures experiments from two assays. BMC Bioinformatics 13, 325 (2012).

96. Wu X, Hasan MA, Chen JY. Pathway and network analysis in proteomics. J Theor Biol, in press (2014).

97. Wei AH, Schoenwaelder SM, Andrews RK, Jackson SP. New insights into the haemostatic function of platelets. Br J Haematol 147, 415-430 (2009).

98. Westrick RJ, Winn ME, Eitzman DT. Murine models of vascular thrombosis. Arterioscler Thromb Vasc Biol 27, 2079-2093 (2007).

99. Borissoff JI, Spronk HMH, ten Cate H. The hemostatic system as a modulator of atherosclerosis. N Engl J Med 364, 1746-1760 (2011).

100. Denis CV, Dubois C, Brass LF, Heemskerk JW, Lenting PJ, Biorheology Subcommittee of the SCC of the ISTH. Towards standardization of in vivo thrombosis studies in mice. J Thromb Haemost 9, 1641-1644 (2011). 


\section{CHAPTER 2}

\section{MONITORING IN VITRO \\ THROMBUS FORMATION WITH \\ NOVEL MICROFLUIDIC DEVICES}

Westein E, de Witt SM, Lamers M, Cosemans JM, Heemskerk JW 


\begin{abstract}
Cardiovascular disease is a major cause of mortality globally and is subject to ongoing research to improve clinical treatment. It is established that activation of platelets and coagulation are central to thrombosis, yet at different extents in the arterial and venous system. In vitro perfusion chamber technology has contributed significant knowledge on the function of platelets in the thrombotic process under shear conditions. Recent efforts to downscale this technique with a variety of microfluidic devices has opened new possibilities to study this process under precisely controlled flow conditions. Such microfluidic devices possess the capability to execute platelet function tests more quickly than current assays, while using small blood samples. Gradually becoming available to the clinic now, they may provide a new means to manage the treatment of cardiovascular diseases, although accurate validation studies still are missing. This review highlights the progress that has been made in monitoring aspects of thrombus formation using microfluidic devices.
\end{abstract}

\title{
First generation fluidic devices to assess platelet function under flow
}

Given the established role of platelets in normal hemostasis and in pathological thrombosis, many assay systems have been developed to determine mechanisms underlying platelet (dys)function. The origin of platelet testing can be traced back to the early 1900s, when Duke established the in vivo bleeding time as a measure of platelet function ${ }^{1}$. Nowadays, less invasive platelet function tests are routinely used for the diagnosis and management of patients with platelet dysfunctions as well as for monitoring the efficacy of antiplatelet therapies, such as with aspirin and purinergic receptor antagonists. Of more recent interest is the use of platelet function testing as a tool to aid the prediction of bleeding complications during surgery and to guide postsurgical haemostatic therapies.

A general limitation of the most platelet function assays is that they do not well correlate with the clinical outcome in terms of bleeding or thrombosis. As described in recent overviews, the use of flow chamber technology has promising potential to overcome this limitation ${ }^{2,3}$. Conventional platelet function assays mostly exclude coagulation and only few assays introduce shear stress as an additional parameter (e.g. PFA-100). On the other hand, flow perfusion chambers allow the incorporation of platelet function, coagulation and shear stress at the same time. In addition, they allow the use of various platelet-adhesive substrates as a surface, most typically collagen type I/ III, von Willebrand factor (VWF), fibrinogen, tissue factor, fibronectin, extracellular matrix from endothelium, and human atherosclerotic plaque ${ }^{4,5,6}$. Baumgartner pioneered the first successful in vitro flow device, the annular perfusion chamber in which denuded and inverted arterial segments were used as an adhesive surface ${ }^{7}$. Later, Sakariassen et al. developed a parallel-plate perfusion chamber model in which a glass coverslip is coated with an adhesive substrate or endothelial cells and thrombus formation can be evaluated at shear rates of 50 to $10,500 \mathrm{~s}^{-18}$. This model has also been used in the study of interactions of leukocytes with endothelium. To date, the majority of perfusion chambers still use the same parallel-plate principle to study mechanisms of platelet adhesion and aggregation over a large range of shear rates ${ }^{9}$. These systems have uncovered a multitude of pathways leading to $\mathrm{Ca}^{2+}$ signaling, integrin activation and secretion which contribute to thrombus formation and are tightly regulated by the hemodynamic environment $10,11,12$.

Although flow chamber technology with parallel-plate flow chambers is a proven tool to test platelet function and anti-thrombotic therapies, it is yet to be introduced into mainstream clinical diagnostic practise, mainly due to a lack of standardization in chamber construction and the use of this method ${ }^{13}$. Further limitations are that these assays mostly rely on complex microscopic imaging analysis and require highly trained personnel. In addition, the volume of blood needed to perform multiple perfusions can be quite large; typically $5 \mathrm{~mL}$ or more, depending on the dimensions of the perfusion chamber and the required wall shear rate ${ }^{14}$. Nowadays, these limitations of in vitro perfusion chambers are being addressed with the use of microfluidic technology. 


\section{New types of microfluidic devices for blood flow perfusion}

As indicated below, the use of microfluidic technology provides new in depth knowledge of specific processes that regulate platelet activation under flow because of the unique and flexible design of such devices and their capability to high-throughput and small-dimension investigations into thrombus formation.

\section{Manufacturing of polydimethylsiloxane microfluidic devices}

Microfluidic technology comprises the design and manufacturing of devices for manipulation of fluids on a micrometer scale. Typically, this technique uses flow channel dimensions of 2-100 $\mu \mathrm{m}$ with fluid volumes inside these devices being in the lower microliter range. Although microfluidic devices can be fabricated with metal, glass or plastics, most devices that are currently used in cell biological research are made from the polymer, polydimethylsiloxane (PDMS). This silicone rubber is easy-to-use, inexpensive, biocompatible and transparent, which make PDMS chambers a cost effective and disposable device to investigate thrombus formation with high optical resolution. As a direct result of this, many different types of PDMS devices are reported, often with increased throughput over single channel devices. An overview of the current use of PMDS and other microfluidics is given in Table 1.

In general, for the construction of PDMS chambers, a photoresist layer, consisting of the epoxy-based, photo-cross-linkable polymer SU-8 is deposited on a silicon substrate and patterned as desired by using conventional lithography ${ }^{15}$. This process involves the placement of an optically dense mask with a pattern of channels, followed by exposure to ultraviolet radiation to harden the SU-8 polymer. After extensive washing, the silicon master plate with a negative of the patterned channels serves as a stiff, reusable mold. A mixture of PDMS oligomers is then poured on this mold, allowed to solidify by cross-linking and then peeled off from the mold. Holes are punctured in the PDMS block to reach the closed channel structure, and tubing is connected to the channels for fluid inlet. The PDMS cast is usually used in combination with a glass coverslip which can be coated with platelet adhesive ligands as desired. The PDMS cast is bonded to the glass either by hydrophobic interaction or by plasma bonding. The result is a flow device with single or multiple channels, as desired (Table 1).

\section{Precisely manipulating the flow conditions}

The ability to computationally predict and accurately control the fluid dynamics in microfluidic devices makes these ideal tools to mimic and study vascular processes that occur at predicted hemodynamic shear conditions ${ }^{9}$. It has been demonstrated that a channel ratio of width to height larger than 10 is desirable for uniform shear stress distribution across the microchannel, thereby yielding similar degrees of platelet adhesion to collagen type I compared to conventional parallel-plate devices ${ }^{16}$. Nevertheless, microfluidic chips are reported with a much larger range of channel dimension ratios. A key characteristic for channels with a low width-height ratio is the appearance of considerable hydrodynamic effects stemming from the sidewalls, which typically lead to increased platelet adhesion in areas near the sidewalls ${ }^{16}$. The impact of sidewall hemodynamics was exploited by Neeves and colleagues, who monitored platelet adhesion in various areas of the flow channel and found a proportional increase in platelet deposition near the sidewalls relative to the centre of the channel that was dependent on the applied input shear rate ${ }^{17}$.

An innovative microfluidic design was recently reported that completely eliminated effects of the channel sidewalls ${ }^{18}$. In this method, the blood sample is hydrodynamically focussed between two layers of buffer, such that it is transported as a cylindrically sheathed stream, which does not interact with the channel side walls (Figure 1A). Due to the presence of laminar flow conditions, dilutional effects are eliminated in this setup, thereby preventing the blood to mix with the adjacent buffer streams. The design is such that the sample volume could be reduced to less than 50 $\mu \mathrm{L}$. Proof of concept of this system was demonstrated in that it showed a $50 \%$ inhibitory effect on 


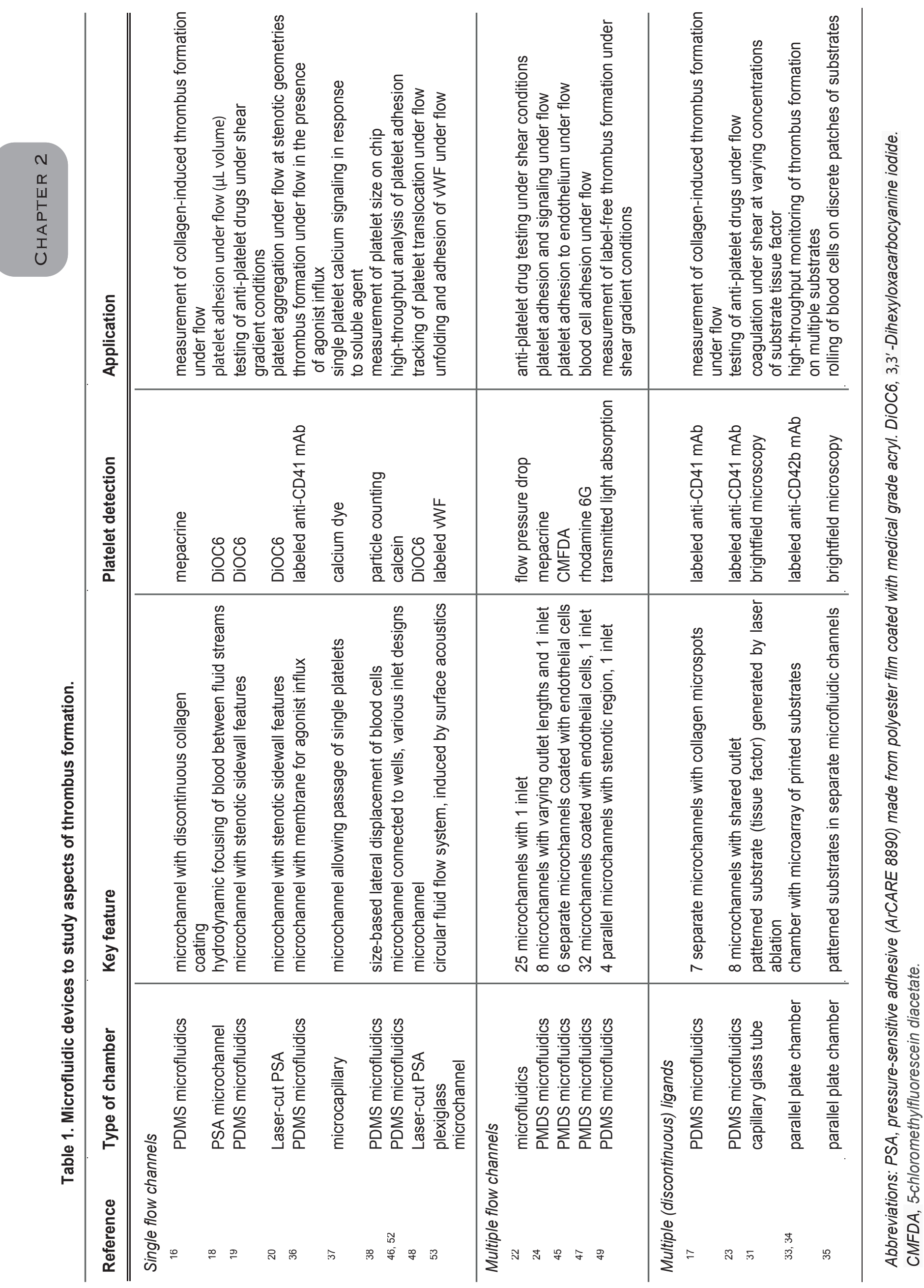


platelet surface adhesion to VWF with the integrin $\mathrm{a}_{\mathrm{IIb}} \beta_{3}$ inhibitor, abciximab.

Microfluidics have also been used to study effects of vessel stenosis on the thrombotic process. Nesbitt and Westein employed a microfluidics system with protruding sidewall features to induce platelet aggregation at the apex of the stenosis ${ }^{19}$. It was demonstrated that platelet aggregation under conditions of micro-shear gradients can occur independently of autocrine platelet stimulation by ADP or thromboxane $A_{2}$. The same group characterized the exact fluid dynamics patterns in microfluidic stenosis channels and showed that the magnitude of micro-shear gradients correlated with the platelet aggregation response ${ }^{20}$.

Microfluidic devices have furthermore been fabricated with circular cross-sections by introducing a coaxial stream of a gas and a solution of silicone oligomer in an organic solvent ${ }^{21}$. The oligomer polymerizes outside the gas stream and leaves a circular cross section after removal of the solvent. This method is designed to mimic vascular conditions, and uses confluently grown endothelial cells, thus replicating a round blood vessel with relevant circular blood flow hemodynamics. Blood perfusion studies using this device have not been reported yet, but will be of significant interest.

\section{Up-scaling the throughput of blood samples}

Perhaps the most promising aspect of microfluidic technology to investigate thrombus formation is the ease and flexibility with which special PDMS microfluidic channel configurations can be designed. This makes the microfluidics technology ideally suited to up-scaling of the throughput by incorporating multiple flow channels into a single perfusion round, while keeping the required blood volume to a minimum. This aspect is a clear advantage of microfluidics over standard parallel-plate flow devices. Hosokawa et al. studied collagen-dependent thrombus formation in 25 parallel capillary channels, which were fed from a single blood sample reservoir (Figure 1B). Using this approach, the efficacy of several antiplatelet drugs (aspirin, ADP receptor antagonists, abciximab) could be evaluated under physiological flow conditions by monitoring flow pressure and by microscopic imaging ${ }^{22}$. In this system dual platelet inhibition with aspirin and $\mathrm{P}_{2} \mathrm{Y}_{12}$ antagonist was more effective in thrombus inhibition than either drug alone. Although the method purposely averaged the flow pressure drop over all 25 channels, it must be noted that fluid flow in individual parallel channels fed from a common inlet reservoir is affected by the flow in other channels, when extensive thrombus formation occurs. This limitation is overcome by restraining the thrombus build-up. In this manner, Maloney et al. investigated the effects of $P_{2} Y_{1}$ and $P 2 Y_{12}$ antagonists on collagen-induced platelet aggregation in a device with 8 parallel channels (Figure $1 \mathrm{C}$ ) ${ }^{23}$. In a single perfusion with less than $100 \mathrm{~mL}$ of blood, the $\mathrm{IC}_{50}$ values of both types of antagonists were determined.

Upscaling of sample throughput has also been done with particular emphasis on the shear dependency of thrombus formation. PDMS devices were developed, allowing blood flow at different shear rates with one sample, reducing inter-assay variability. Figure 1D shows an example of a parallel 8-channel design with different shear rate conditions by varying the lengths, and hence the fluid resistance, of the channel outlets. With this device the shear-dependent contributions of intracellular and extracellular domains of platelet $\mathrm{a}_{\mathrm{IIb}} \beta_{3}$ were determined ${ }^{24}$.

\section{Down-scaling the size of platelet adhesive surfaces}

Another aspect of thrombus formation that can be studied using microfluidics is the effect of changing the adhesive surface, for instance by discontinuous coating procedures of small patches of adhesive ligands. The rationale for this is that endothelial damage in thrombosis is often focussed on in nature, a situation that can be mimicked by discontinuous ligand coating. A practical benefit of such coating is that excessive aggregate growth leading to channel occlusion can be prevented. Various techniques are reported to create local microspots of platelet-adhesive ligands. Photolithography technology is used to create arrays of adhesive microspots surrounded by non- 


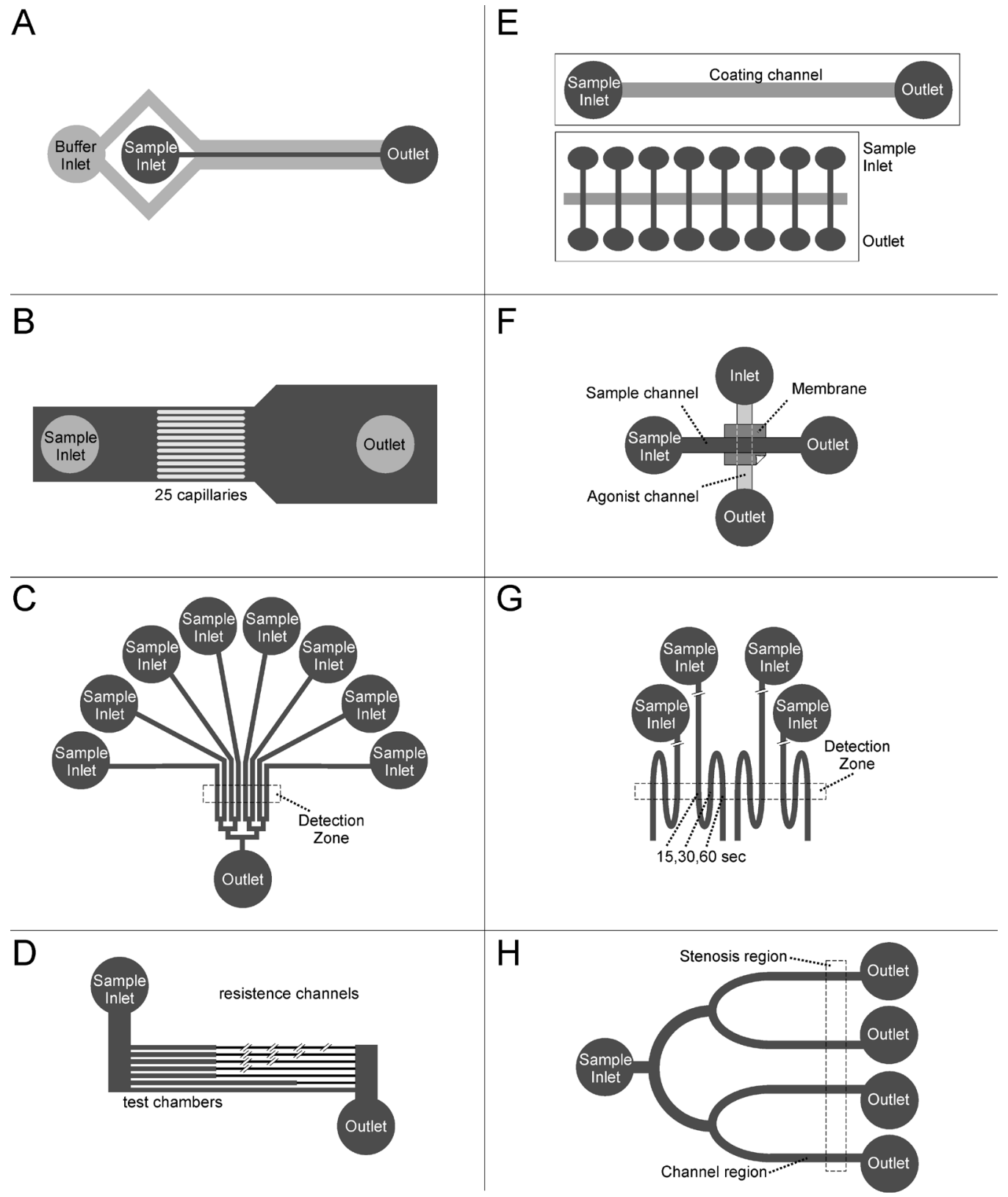

Figure 1. Microfluidic flow devices with special design. (A) Microfluidic device from polymethyl methacrylate (PMMA), consisting of a top plate, a channel of two laser-patterned PSA gaskets for buffer and sample and a coated coverslip. The buffer gasket allows two streams of buffer flowing parallel with the blood sample, resulting in three parallel streams in the main channel. Adapted from ${ }^{18}$. (B) Microchip device (width $40 \mu \mathrm{m}$, height $40 \mu \mathrm{m}$ ) containing 25 micro-capillaries, which is placed on a surface with immobilized collagen. Adapted from ${ }^{22}$. (C) Multi-inlet single outlet flow device with 8 parallel perfusion channels in combination with a common collagen coating. Adapted from ${ }^{23}$. (D) Microfluidic networks containing $24 \mu \mathrm{m}$ deep flow channels. Additional resistance channels vary in length to create different flow rates per flow channel. Channels are connected to a common sample inlet at controlled pressure. Adapted from ${ }^{24}$. (E) Device of parallel microfluidic flow channels (100 $\mu \mathrm{m}$ width) connected to a functionalized coverslip, coated with a perpendicular strip of collagen. Adapted from ${ }^{17}$. (F) Microfluidic device consisting of a top channel with flowing blood, a track-etched polycarbonate membrane and a bottom channel that contains agonists, mounted to a vacuum holder. The cross-section area is $100 \times 100 \mu \mathrm{m}$, where the flux of the agonist molecules is controlled by the membrane. Adapted from ${ }^{36}$. (G) Caliper Technologies FS-417 four-sipper cell chip designed and used to detect agonist-induced calcium flux. Samples are drawn from the four "cell wells" and streamed through the chip. Agonist mixing is performed by diffusion while sample is continuously flown through the chip past the fluorescence detection zone. Flow rate is dependent on the combination of applied vacuum and the viscosity of the sample. Adapted from ${ }^{37}$. (H) Microfluidic system designed to induce platelet aggregation at four distinct shear rates in whole blood within a high shear stenotic region (single inlet, multi-outlet). An optical system measures light transmission in the stenotic region. Adapted from ${ }^{23}$. 
adhesive proteins or a lipid bilayer $25,26,27$. The size and shape of the spots is controlled by photolithography etching after placing a photomask with desired features and by irradiation with deep ultraviolet light. This method has been used to create circular microspots of phospholipids containing tissue factor ${ }^{28}$. It was elegantly demonstrated that, in addition to the absolute protein density, the size and the spatial arrangement of the microspots are critical in the ability of tissue factor to support coagulation ${ }^{29}, 30$. Using a similar technique, a $200 \mathrm{~mm}$ patch of phospholipids containing tissue factor was immobilized in a circular glass capillary tube and characterized for coagulation under flow conditions ${ }^{31}$. Phospholipid containing TF has recently been used in combination with immobilized collagen type I to study the stabilizing effects of fibrinformation on platelet aggregates 32. By monitoring embolization events in the microfluidic channels it was found that fibrin increased clot strength by 12 - to 28 -fold.

PDMS-based devices can also be used for micro-contact printing, which presents another method to deliver discrete patches of adhesive ligands to a glass surface ${ }^{25}$. This method makes use of the high protein adsorption properties of PDMS. Ligands like collagen are incubated on a PDMS stamp with desired feature and are then transferred to glass by briefly stamping the PDMS block onto the glass surface. After blood perfusion, this results in thrombus formation precisely at the stamped areas (Figure 2A).

Alternatively, immobilizing discrete ligand patches can be achieved with microarray printing. Diamond and co-workers describe that microarray printing of collagen and VWF, using $176 \mu \mathrm{m}$ wide microspots in which multiple ligand combinations were studied in a single blood perfusion run 33. This same technology was also employed to print microarray spots of collagen containing va rious amounts of tissue factor to determine threshold concentrations of triggers for coagulation in whole blood ${ }^{34}$. Calculations learned that about 10 molecules tissue factor per $\mathrm{mm}^{2}$ are sufficient to initiate fibrin formation at $1000 \mathrm{~s}^{-1}$ shear rate. Although microarray printing has clear advantages, the relatively large diameter of the microspots commonly used $(100-1000 \mathrm{~mm})$ limits its application with small microfluidic systems. Hence, micro-spotting is usually performed in combination with conventional parallel plate flow devices.

Discrete ligand patches can also be immobilized in microfluidic devices by infusing the ligand solution through separate microfluidic "coating" channels with defined width and allow the ligand to adsorb to the glass surface. Following removal of these channels, a second set of microfluidic channels is placed perpendicular to the direction of the first channel (Figure 2B). After blood perfusion, this results in thrombus formation selectively at the discrete coating strips. Using a similar setup it was demonstrated that PAR4 agonists stimulate platelet aggregation at the collagen surface (Figure $1 \mathrm{E}$ ) ${ }^{17}$. This approach has also been used by others, who made immobilized strips of P-selectin, E-selectin and VWF, perpendicular or parallel to the direction of blood flow, to study the rolling velocity of neutrophils and platelets ${ }^{35}$.

\section{Novel applications of microfluidic devices for platelet and vascular research}

Novel microfluidic techniques have been used to elucidate platelet and endothelial cell functions under flow conditions.

\section{Platelet activation under flow}

Using sophisticated PDMS microfluidics, the spatio-temporal role of ADP activation during thrombus formation under flow was investigated ${ }^{36}$. Via two perpendicular channels, separated by a porous membrane, ADP was allowed to flux in a controlled way from the bottom channel into the upper perfusion channel (Figure 1F). It was demonstrated that the density of platelets within an aggregate increased with increasing ADP flux. In other work, a multi-channel system was used to simultaneously measure multiple concentrations of inhibitors of ADP-dependent platelet aggregation on a collagen surface, demonstrating that apyrase lacks inhibitory function under high shear conditions ${ }^{23}$. 
Microfluidics can also monitor the activation of single platelets in suspension, e.g. by detecting $\mathrm{Ca}^{2+}$ responses following on-chip mixing with agonists. By constructing a channel with a narrowing in the detection zone, individual responding platelets could be detected while passing through the channel (Figure 1G). This method demonstrated increases in intracellular $\mathrm{Ca}^{2+}$ levels following stimulation by the PAR-1 agonist SFLLRN, or ADP ${ }^{37}$. An innovative microfluidic method to measure label-free platelet activation is based on the principle of deterministic lateral displacement to separate platelets on-chip from other cell types based on their size ${ }^{38}$. This method exploits the fact that small particles have a stronger lateral displacement than larger particles in a fluid flow.

The degree of displacement was measured separating the fluid flow into multiple parallel streams and counting platelet density in each parallel channel. This method proved to be accurate enough to detect the increased platelet size associated with thrombin activation.

\section{Endothelial cell functions under flow}

The endothelium has well characterized inhibitory mechanisms to prevent platelet activation through the production of nitric oxide and prostacyclin. On the other hand, VWF and P-selectin, stored in and secreted from the endothelial Weibel-Palade bodies, are potent ligands to recruit platelets and leukocytes. This places endothelial cells in a central position to regulate platelet function

A
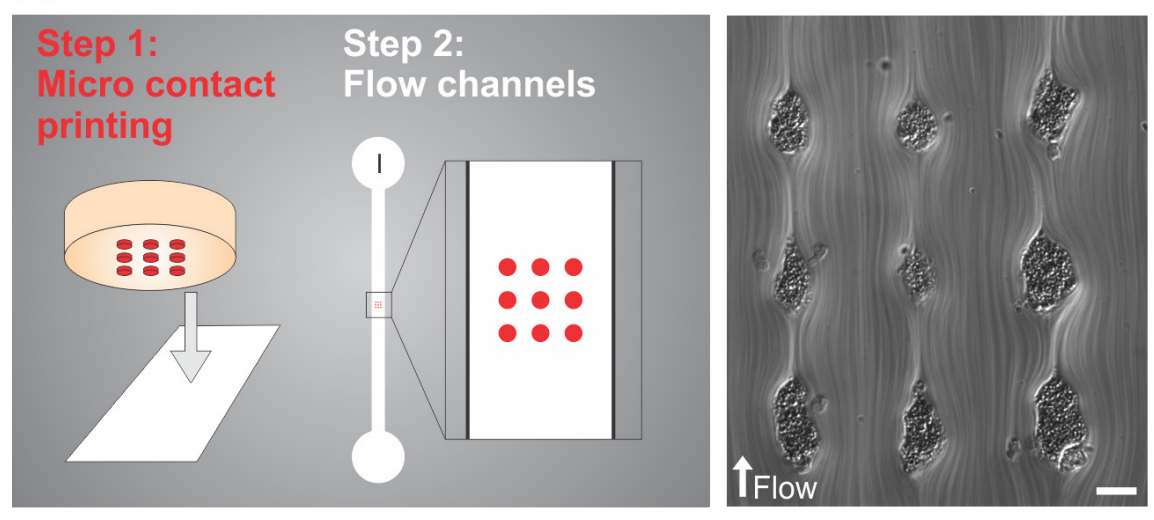

B
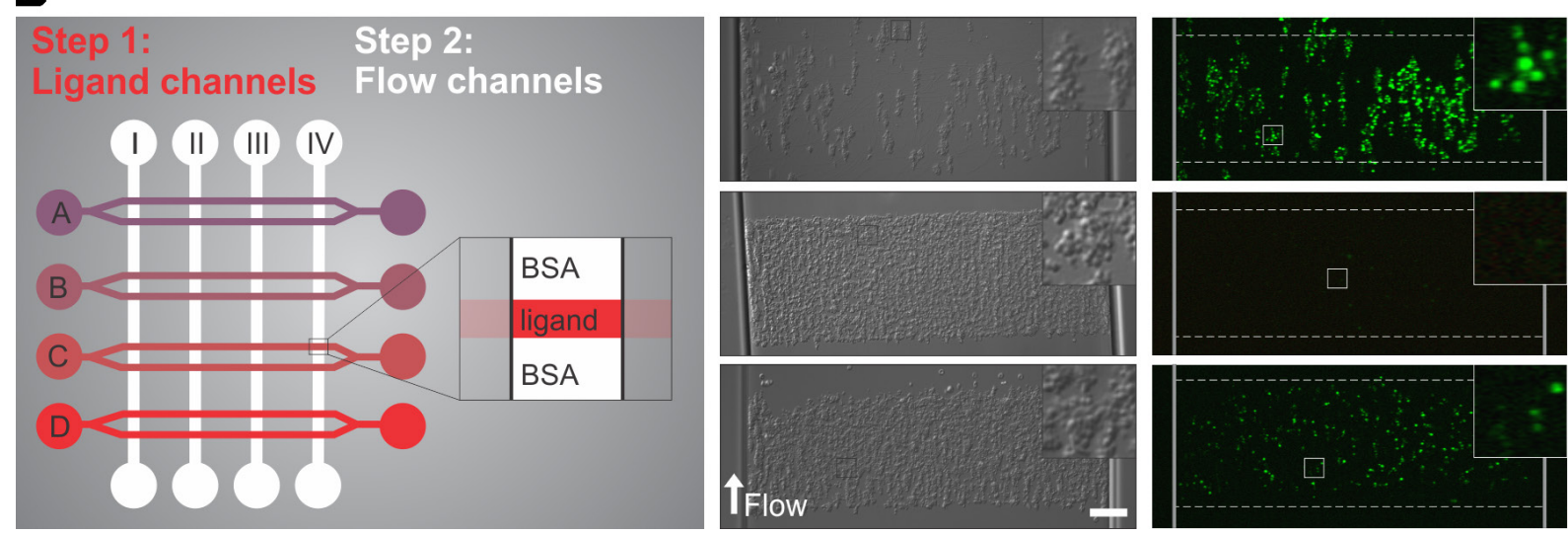

Figure 2. High-throughput flow microfluidics with discontinuously immobilized ligands. (A) Step 1: Micro-contact printing of collagen type III onto a glass coverslip, giving spot sizes of $20 \mathrm{~mm}$. Step 2: A PDMS flow channel is placed over the microspot pattern and whole blood perfusion is done at $1600 \mathrm{~s}^{-1}$. The result is platelet adhesion specifically on the collagen-coated microspots. Scalebar, $20 \mathrm{~mm}$. (B) Step 1: 4 PDMS coating channels with dual configuration (175 $\mu \mathrm{m}$ width) are used to immobilize up to 4 ligands on the glass resulting in $4 \times 2$ coated strips. Step 2: A second PDMS device with 4 independent flow channels (I to IV) is placed perpendicular over the coated strips, allowing thrombus formation in a multi-ligand perfusion. Right panels: Platelet adhesion to collagen type I (top), fibrinogen (middle) and collagen type III (bottom). Platelets and phosphatidyl serine exposure are visualized with brightfield and fluorescence microscopy respectively. Insets: zoomed area. Scale bar, $50 \mu \mathrm{m}$. 
and justifies their use in microfluidics to more closely mimic the physiological environment in which platelets can adhere.

Several flow assays focus on shear stress effects in relation to endothelial cell function, such as tests for cytoskeletal remodelling and protein secretion mechanisms $39,40,41$. Chau and colleagues demonstrated that shear stress triggers secretion of VWF from human umbilical vein endothelial cells cultured in a multichannel microfluidic device ${ }^{42}$. Similar systems have been used to study the endothelial migration response through a chemo-attractant gradient. Microfluidic devices have the exclusive possibility to create a fluid gradient due to their laminar flow characteristics, which prevent mixing of two adjacent fluid streams. This property has been exploited by some groups to study the migration effects of endothelial cells through an immobilized chemo-attractant gradient that was accomplished by introducing parallel streams of different chemotactic agents ${ }^{43,44}$.

The use of endothelial cells in microfluidic systems in combination with blood perfusion is only just emerging. A first paper, focussing on direct interactions of platelets with endothelial cells, demonstrated a role for ADP in platelet adhesion to endothelial cells, particularly after inhibition of endothelial nitric oxide production ${ }^{45}$. However, this study was performed under static conditions, and the translation to flow conditions has not been made. The feasibility of microfluidics with seeded endothelial cells to monitor results of blood flow was demonstrated by Conant et al. ${ }^{46}$, who showed stable platelet adhesion and thrombus formation after endothelial stimulation with TNF-a. PDMS microfluidics using multiple parallel microchannels, lined with endothelial cells, could also model the pathophysiological processes of sickle cell disease and haemolytic uremic syndrome under blood flow conditions ${ }^{47}$.

\section{New detection technologies in combination with microfluidic devices}

Detection methods in microfluidic devices are typically dedicated to monitor platelet adhesion and aggregation events, similarly to techniques used with parallel-plate flow chambers ${ }^{14}$. The most common applied technique is brightfield, contrast-enhanced microscopy, visualizing flow-adhered platelets in real time at high optical resolution. Microscopy readouts of microfluidic flow assays report on platelet density, ranging from individual platelet behaviour to large scale thrombus formation (Table 1). Alternative approaches are the detection of platelets by labeling with DiOC6 or fluorescently labeled antibodies. Tracking software has been used to monitor the translocation of platelets on a VWF surface ${ }^{48}$. However, robust automated image analysis procedures for real-time quantification of thrombus formation still need to be developed.

Alternative detection techniques are being explored to characterize platelet adhesion and aggregation by measuring light absorbance. Transmitted light can quantify platelet-rich thrombi due to the displacement of red blood cells leading to an increase in transmittance ${ }^{49}$. However, this detection method cannot inform on platelet activation levels or thrombus density. Another method to detect platelet deposition with high sensitivity uses a quartz crystal microbalance ${ }^{50}$. This method relies on the resonance frequency of a crystal in an alternating electric field, which is modulated by mass deposition at the detector surface. This detection modality could monitor effects of ADP stimulation and integrin $\mathrm{a}_{\mathrm{IIb}} \beta_{3}$ inhibition of platelets on a fibrinogen surface. Also this detection does not provide qualitative information on thrombus composition. Nevertheless, it is to be expected that microfluidic platelet function tests will benefit from current developments in sensitive detection methods.

\section{Microfluidic device development towards preclinical testing}

Microfluidic technology is regularly used in clinical routines, but this is limited to biochemical measurements of specific blood components. Platforms such as Spinit, Asklepios, Kumetrix, Fluidics -on-Flex and Piccolo Xpress have been developed for point-of-care diagnostics detecting a large range of proteins, lipids and electrolytes in the nano- to micromolar range. However, the use of microfluidic devices for monitoring platelet aggregation and thrombus formation is still limited to 
the academic setting, in the majority of cases for proof-of-principle experiments. An attempt to use PDMS microfluidics devices for whole-blood testing of thrombus formation in a point-of-care setting has been presented by Li et al. ${ }^{49}$. These authors used a chip with 4 parallel microchannels with stenotic region (shear rates $500-13,000 \mathrm{~s}^{-1}$ ) to measure label-free thrombus formation by light absorption (Figure $1 \mathrm{H}$ ). This method consumes blood without labeling, is fast and is relatively easy to perform. However, the assay is not yet validated for clinical applications, and its sensitivity to detect platelet hypo- or hyper-function is unknown. Limitations of this flow test are the relatively large blood sample volumes $(2-8 \mathrm{~mL})$, the inability to check for platelet activation markers and the use of only one adhesive substrate. The challenge will be to correctly integrate aspects from this and other microfluidics protocols to a test that is robust and sensitive enough to detect aberrant platelet function in a clinical setting.

An important reason for further development of microfluidic devices for pre-clinical research and patient diagnosis is that they can provide another approach in comparison to the current assays that target platelet activation in a point-of-care setting (Multiplate aggregometry, VerifyNow, Impact, PlateletWorks, PFA-100, Cone-and-Plate Analyzer). As reviewed elsewhere, the diagnostic and prognostic value of these assays is only moderate (Review Harisson; this issue of Platelets). This is in part due to the multiplicity of platelet activation and regulatory pathways, which are difficult to capture in a single function test. For example, light transmittance aggregometry, VerifyNow, PlateletWorks, and PFA provided only limited prognostic information to identify patients at higher risk of bleeding following stent implantation ${ }^{51}$. The clear advantage of PDMS microfluidic devices is that, using a small amount of blood, they can provide a means for high-throughput analysis of platelet function in the natural environment of flowing blood, at a range of shear rates and a variety of platelet-adhesive substrates. Especially in combination with multiple ligand micro-spotting, microfluidics have the potential to be developed as a clinical diagnostic tool for platelet function that may compete with other point-of-care tests.

\section{Conclusions}

Platelet activation in the in vivo environment is regulated by differentially exposed extracellular matrix components, complex rheological flow conditions and release or generation of soluble platelet agonists. Microfluidics have made it possible to investigate thrombus formation in a multi-variable manner with accurate control of wall shear rate conditions with small blood samples, while using multichannel perfusion and microspot ligand coating. Another important possibility is the use of multiple ligands in a single perfusion, allowing increased throughput and determination of ligand interactions. In comparison to current point-of-care function tests, microfluidic assays may take the complex hemodynamic and biochemical environments present in vivo better into account. Integration of all these parameters into a user friendly device will aid in the management of cardiovascular diseases by improving current platelet function tests.

\section{Acknowledgements}

EW holds an Australian NHMRC Overseas Training Fellowship, I.D. 606742. This work was supported by the Centre for Translational Molecular Medicine (CTMM) INCOAG and the Netherlands Heart Foundation (2011T006).

\section{References}

1. Duke WW. The relation of blood platelets to hemorrhagic disease. Description of a method for determining the bleeding time and the coagulation time and report of three cases of hemorrhagic disease relieved by blood transfusion. JAMA 55, 1185-1192 (1910).

2. Zwaginga JJ, Nash G, Heemskerk JW, Frojmovic M, Hoylaerts MF, Sakariassen KS. Flow-based assays for global assessment of hemostasis. Part 1: Biorheologic considerations. J Thromb Haemost 4, 24862487 (2006). 
3. Zwaginga JJ, Sakariassen KS, Nash G, King MR, Heemskerk JW, Frojimovic M, Hoylaerts MF. Flowbased assays for global assessment of hemostasis. Part 2: current methods and considerations for the future. J Thromb Haemost 4, 2716-2717 (2006).

4. $\quad$ Ruggeri ZM. Platelets in atherothrombosis. Nat Med 8, 1227-1234 (2002).

5. Heemskerk JW, Sakariassen KS, Zwaginga JJ, Brass LF, Jackson SP, Farndale RW. Collagen surfaces \ to measure thrombus formation under flow: possibilities for standardization. J Thromb Haemost 9, 856-858 (2011).

6. Cosemans JM, Kuijpers MJ, Lecut C, Loubele ST, Heeneman S, Jandrot-Perrus M, Heemskerk JW. Contribution of platelet glycoprotein VI to the thrombogenic effect of collagens in fibrous atherosclerotic lesions. Atherosclerosis 181, 19-27 (2005).

7. Sakariassen KS, Hanson SR, Cadroy Y. Methods and models to evaluate shear-dependent and surface reactivity-dependent antithrombotic efficacy. Thromb Res 104, 149-174 (2001).

8. Sakariassen KS, Muggli R, Baumgartner HR. Measurements of Platelet Interaction with Components of the Vessel Wall in Flowing Blood. Methods in Enzymology 169, 37-70 (1989).

9. Jackson SP, Nesbitt WS, Westein E. Dynamics of platelet thrombus formation. J Thromb Haemost 7 Suppl 1, 17-20 (2009).

10. Munnix IC, Kuijpers MJ, Auger J, Thomassen CM, Panizzi P, van Zandvoort MA, Rosing J, Bock PE, Watson SP, Heemskerk JW. Segregation of platelet aggregatory and procoagulant microdomains in thrombus formation: regulation by transient integrin activation. Arterioscler Thromb Vasc Biol 27, 2484-2490 (2007).

11. Cosemans JM, Iserbyt BF, Deckmyn $\mathrm{H}$, Heemskerk JW. Multiple ways to switch platelet integrins on and off. J Thromb Haemost, 6: 1253-61 (2008).

12. Jackson SP. The growing complexity of platelet aggregation. Blood 109, 5087-5095 (2007).

13. Roest M, Reininger A, Zwaginga JJ, King MR, Heemskerk JWM. The Biorheology Subcommittee of the SSCotI. Flow chamber-based assays to measure thrombus formation in vitro: requirements for standardization. J Thromb. Haemost 9, 2322-2324 (2011).

14. Van Kruchten R, Cosemans JM, Heemskerk JW. Measurement of whole blood thrombus formation using parallel-plate flow chambers - a practical guide. Platelets 23, 229-242 (2012).

15. Duffy DC, McDonald JC, Schueller OJ, Whitesides GM. Rapid Prototyping of Microfluidic Systems in Poly (dimethylsiloxane). Anal Chem 70, 4974-4984 (1998).

16. Sarvepalli DP, Schmidtke DW, Nollert MU. Design considerations for a microfluidic device to quantify the platelet adhesion to collagen at physiological shear rates. Ann Biomed Eng 37, 1331-1341 (2009).

17. Neeves KB, Maloney SF, Fong KP, Schmaier AA, Kahn ML, Brass LF, Diamond SL. Microfluidic focal thrombosis model for measuring murine platelet deposition and stability: PAR4 signaling enhances shear-resistance of platelet aggregates. J Thromb Haemost 6: 2193-01 (2008).

18. Kent NJ, O'Brien S, Basabe-Desmonts L, Maeda GR, MacCraith BD, Corcoran BG, Kenny D, Ricco AJ. Shear-mediated platelet adhesion analysis in less than 100 mul of blood: toward a POC platelet diagnostic. IEEE Trans Biomed Eng 58, 826-830 (2011).

19. Nesbitt WS, Westein E, Tovar-Lopez FJ, Tolouei E, Mitchell A, Fu J, Carberry J, Fouras A, Jackson SP. A shear gradient-dependent platelet aggregation mechanism drives thrombus formation. Nat Med 15, 665-673 (2009).

20. Tovar-Lopez FJ, Rosengarten G, Westein E, Khoshmanesh K, Jackson SP, Mitchell A, Nesbitt W. A microfluidics device to monitor platelet aggregation dynamics in response to strain rate microgradients in flowing blood. Lab Chip 10, 291-02 (2010).

21. Fiddes LK, Raz N, Sgrigunapalan S, Tumarkan E, Simmons CA, Wheeler AR, Kumacheva E. A circular cross-section PDMS microfluidics system for replication of cardiovascular flow conditions. Biomaterials 31, 3459-3464 (2010).

22. Hosokawa K, Ohnishi T, Fukasawa M, Kondo T, Sameshima H, Koide T, Tanaka KA, Maruyama I. A microchip flow-chamber system for quantitative assessment of the platelet thrombus formation process. Microvasc Res 83, 154-61 (2011).

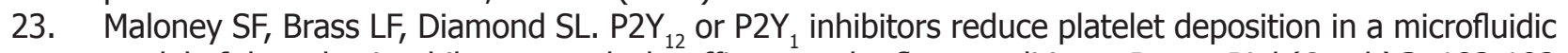
model of thrombosis while apyrase lacks efficacy under flow conditions. Integr Biol (Camb) 2, 183-192 (2010).

24. Gutierrez E, Petrich BG, Shattil SJ, Ginsberg MH, Groisman A, Kasirer-Friede A. Microfluidic devices for studies of shear-dependent platelet adhesion. Lab Chip 8, 1486-1495 (2008).

25. Thery M, Piel M. Adhesive micropatterns for cells: a microcontact printing protocol. Cold Spring Harb Protoc, prot5255 (2009).

26. Azioune A, Carpi N, Tseng Q, Thery M, Piel M. Protein micropatterns: A direct printing protocol using 
deep UVs. Methods Cell Biol 97, 133-146 (2010).

27. Azioune A, Storch M, Bornens M, Thery M, Piel M. Simple and rapid process for single cell micropatterning. Lab Chip 9, 1640-1642 (2009).

28. Kastrup CJ, Runyon MK, Shen F, Ismagilov RF. Modular chemical mechanism predicts spatiotemporal dynamics of initiation in the complex network of hemostasis. Proc Natl Acad Sci USA 103, 1574715752 (2006).

29. Kastrup CJ, Shen F, Runyon MK, Ismagilov RF. Characterization of the threshold response of initiation of blood clotting to stimulus patch size. Biophys J 93, 2969-2977 (2007).

30. Shen $\mathrm{F}$, Kastrup $\mathrm{CJ}$, Ismagilov RF. Using microfluidics to understand the effect of spatial distribution of tissue factor on blood coagulation. Thromb Res 122 Suppl 1, S27-30 (2008).

31. Shen F, Kastrup $\mathrm{CJ}$, Liu Y, Ismagilov RF. Threshold response of initiation of blood coagulation by tissue factor in patterned microfluidic capillaries is controlled by shear rate. Arterioscler Thromb Vasc Biol 28, 2035-2041 (2008).

32. Colace T, Muthard R, Diamond SL. Thrombus Growth and Embolism on Tissue Factor-Bearing Collagen Surfaces Under Flow: Role of Thrombin With and Without Fibrin. Arterioscler Thromb Vasc Biol 32, 1466-76 (2012).

33. Okorie UM, Diamond SL. Matrix protein microarrays for spatially and compositionally controlled microspot thrombosis under laminar flow. Biophys J 91, 3474-3481 (2006).

34. Okorie UM, Denney WS, Chatterjee MS, Neeves KB, Diamond SL. Determination of surface tissue factor thresholds that trigger coagulation at venous and arterial shear rates: amplification of $100 \mathrm{fM}$ circulating tissue factor requires flow. Blood 111, 3507-3513 (2008).

35. Nalayanda DD, Kalukanimuttam M, Schmidtke DW. Micropatterned surfaces for controlling cell adhesion and rolling under flow. Biomed Microdevices 9, 207-214 (2007).

36. Neeves KB, Diamond SL. A membrane-based microfluidic device for controlling the flux of platelet agonists into flowing blood. Lab Chip 8, 701-709 (2008).

37. Tran L, Farinas J, Ruslim-Litrus L, Conley PB, Muir C, Munnelly K, Sedlock DM, Cherbavac DB. Agonistinduced calcium response in single human platelets assayed in a microfluidic device. Anal Biochem 341, 361-368 (2005).

38. Inglis DW, Morton KJ, Davis JA, Zieziulewicz TJ, Lawrence DA, Austin RH, Sturm JC. Microfluidic device for label-free measurement of platelet activation. Lab Chip 8, 925-931 (2008).

39. Schaff UY, Xing MM, Lin KK, Pan N, Jeon NL, Simon SI. Vascular mimetics based on microfluidics for imaging the leukocyte--endothelial inflammatory response. Lab Chip 7, 448-456 (2007).

40. Song JW, Gu W, Futai N, Warner KA, Nor JE, Takayama S. Computer-controlled microcirculatory support system for endothelial cell culture and shearing. Anal Chem 77, 3993-3999 (2005).

41. Shao J, Wu L, Wu J, Zheng Y, Zhao H, Jin Q, Zhao J. Integrated microfluidic chip for endothelial cells culture and analysis exposed to a pulsatile and oscillatory shear stress. Lab Chip 9, 3118-3125 (2009).

42. Chau L, Doran M, Cooper-White J. A novel multishear microdevice for studying cell mechanics. LabChip 9, 1897-1902 (2009).

43. Barkefors I, Le Jan S, Jakobsson L, Heijl E, Carlson G, Johansson H, Jarvius J, Park JW, Li Jeon N, Kreuger J. Endothelial cell migration in stable gradients of vascular endothelial growth factor $A$ and fibroblast growth factor 2: effects on chemotaxis and chemokinesis. J Biol Chem 283, 13905-13912 (2008).

44. Shamloo A, Ma N, Poo MM, Sohn LL, Heilshorn SC. Endothelial cell polarization and chemotaxis in a microfluidic device. Lab Chip 8, 1292-1299 (2008).

45. Ku CJ, D'Amico Oblak T, Spence DM. Interactions between multiple cell types in parallel microfluidic channels: monitoring platelet adhesion to an endothelium in the presence of an anti-adhesion drug. Anal Chem 80, 7543-7548 (2008).

46. Conant CG, Schwartz MA, Beecher JE, Rudoff RC, Ionescu-Zanetti C, Nevill JT. Well plate microfluidic system for investigation of dynamic platelet behavior under variable shear loads. Biotechnol Bioeng 108, 2978-2987 (2011).

47. Tsai M, Kita A, Leach J, Rounsevell R, Huang JN, Moake J, Ware RE, Fletcher DA, Lam WA. In vitro modeling of the microvascular occlusion and thrombosis that occur in hematologic diseases using microfluidic technology. J Clin Invest 122, 408-418 (2012).

48. Lincoln B, Ricco AJ, Kent NJ, Basabe-Desmonts L, Lee LP, MacCraith BD, Kenny D, Meade G. Integrated system investigating shear-mediated platelet interactions with von Willebrand factor using microliters of whole blood. Anal Biochem 405, 174-183 (2010).

49. Li M, Ku DN, Forest CR. Microfluidic system for simultaneous optical measurement of platelet aggregation at multiple shear rates in whole blood. Lab Chip 12, 1355-1362 (2012). 
50. Sinn S, Muller L, Drechsel H, Wandel M, Northoff H, Ziemer G, Wendel HP Gehring FK. Platelet aggregation monitoring with a newly developed quartz crystal microbalance system as an alternative to optical platelet aggregometry. Analyst 135, 2930-2938 (2010).

51. Breet NJ, van Werkum JW, Bouman HJ, Kelder JC, Ruven HJ, Bal ET, Deneer VH, Harmsze AM, van der Heyden JA, Rensing BJ, Suttorp MJ, Hackeng CM, ten Berg JM. Comparison of platelet function tests in predicting clinical outcome in patients undergoing coronary stent implantation. JAMA 303, 754-762 (2010).

52. Conant CG, Nevill JT, Zhou Z, Dong JF, Schwartz MA, Ionescu-Zanetti C. Using well-plate microfluidic devices to conduct shear-based thrombosis assays. J Lab Autom 16, 148-152 (2011).

53. Schneider SW, Nuschele S, Wixforth A, Gorzelanny C, Alexander-Katz A, et al. Shear-induced unfolding triggers adhesion of von Willebrand factor fibers. Proc Natl Acad Sci USA 104, 7899-7903 (2007). 



\section{CHAPTER 3}

\section{MULTI-PARAMETER ASSESSMENT OF THROMBUS FORMATION ON MICROSPOTTED ARRAYS OF THROMBOGENIC SURFACES}

De Witt SM, Swieringa F, Heemskerk JW, Cosemans JM 


\begin{abstract}
Thrombus formation by adhering and aggregating blood platelets is fundamental to hemostasis and is a prerequisite for vascular occlusion in pathological thrombosis. The parallel-plate flow chamber technique has been extensively used to measure platelet adhesion and activation in vitro at arterial or venous flow conditions. Here, we describe the use of brightfield and confocal fluorescence microscopy to record the various platelet activation processes contributing to thrombus formation on microspotted arrays of thrombogenic surfaces; and we give procedures to analyze the acquired microscopic images. Furthermore, we describe technical problems that can be expected using the microspot technique. Content: (A) Flow chamber preparation and whole blood perfusion. (B) Brightfield and fluorescence microscopic imaging of thrombi. (C) Analysis of brightfield and fluorescence images.
\end{abstract}

\title{
Introduction
}

Thrombus formation by adhering and aggregating blood platelets is fundamental to hemostasis and is a prerequisite for vascular occlusion in pathological thrombosis ${ }^{1}$.The parallel-plate flow chamber technique has been extensively used to measure platelet adhesion and activation in vitro at arterial or venous flow conditions ${ }^{2-4}$. However, current tests use collagen as the only platelet-adhesive surface, thereby disregarding the contribution of other platelet-adhesive components in the vascular matrix. This is a relevant issue, since multiple platelet adhesive receptors need to interact and signal to form a stable platelet thrombus ${ }^{5-6}$. On type I collagen fibers, the two collagen receptors, glycoprotein VI (GPVI) and integrin $a_{2} \beta_{1}$ (GPIa/IIa) interact with the receptors for von Willebrand factor (VWF), a plasma protein that avidly binds to collagen, i.e. GPIb-V-IX and integrin $a_{\mathrm{IIb}} \beta_{3}$ (GPIIb/IIIa) $)^{6-8}$. It is hence relevant to compare the roles of these receptors with other ones, such as CLEC-2, CD36 (GPIV), and the integrins $a_{5} \beta_{1}, a_{6} \beta_{1}$ and $a_{v} \beta_{3}$. In the accompanying paper, we have described a microspot technology, in which various platelet-adhesive compounds can be coated simultaneously in a flow chamber, and directly compared for their potential to support thrombus formation using small blood samples ${ }^{9}$.

When forming a thrombus, platelets show different types of responses, all of which may contribute to effective hemostasis and pathological thrombosis. These include shape change (pseudopod and lamellipod formation), integrin activation, secretion of the contents of dense granules and a-granules (P-selectin exposure), and actin-dependent contraction of the formed thrombus. ${ }^{1,5}$ In addition, a subpopulation of the platelets - with high cytosolic $\mathrm{Ca}^{2+}-$ assumes a balloon-type of morphology and exposes the procoagulant phospholipid phosphatidylserine (PS) at their outer surface, at which coagulation factors bind and thrombin can be formed ${ }^{10}$.

Since it is unclear how these different platelet responses relate during thrombus formation, we developed procedures to measure these in a systematic way in combination with the microspot technology. ${ }^{9}$ In this protocol paper, we describe the use of brightfield and confocal fluorescence microscopy to record the various platelet activation processes contributing to thrombus formation; and we give procedures to analyze the acquired microscopic images. We note that all assays are performed in the absence of coagulation. Furthermore, we stress that: due to space restrictions not all details could be given; specific procedures may need adaptation in different laboratories; and that expert knowledge will be required for successful completion of the flow assays. We welcome comments on errors and suggestions for improvement. Content:

A. Flow chamber preparation and whole blood perfusion.

B. Brightfield and fluorescence microscopic imaging of thrombi.

C. Analysis of brightfield and fluorescence images. 


\section{Procedures A. Flow chamber preparation and whole blood perfusion}

\section{Reagents}

- Annexin A5 labeled with Alexa Fluor (AF)647 (Molecular Probes, A23204)

- Bovine serum albumin (BSA) (Sigma Aldrich, A6003)

- $\mathrm{CaCl}_{2}$ (Sigma Aldrich, C1016)

- 3,3' Dihexyloxacarbocyanine iodide ( DiOC $_{6}$ ) (Anaspec, 8984715)

- Ethanol (VWR)

- $\quad$ FITC-labeled anti-CD62P (P-selectin) mAb (Immunotech, A07790)

- $\quad$ Fragmin (Pfizer, 5T1532)

- $\quad D$-Glucose (ACS Reagent)

- $\mathrm{HCl}$ (Sigma Aldrich, $\mathrm{H} 1758)$

- 4-(2-Hydroxyethyl)piperazine-1-ethanesulfonic acid (HEPES) (Sigma Aldrich, H3375)

- $\mathrm{KCl}$ (Sigma Aldrich, P9541)

- $\mathrm{MgCl}_{2}$ (Sigma Aldrich, M8266)

- $\quad D$-Phenylalanyl-L-prolyl-L-arginine chloromethylketone (PPACK) (BioConnect, A58SC201291A)

- $\mathrm{NaCl}$ (Sigma Aldrich)

- Unfractionated heparin (Sigma Aldrich, H3393-1)

- FITC-labeled anti-fibrinogen mAb (WAK Chemie Medical, 64162)

\section{Solutions}

- Blocking buffer: $136 \mathrm{mM} \mathrm{NaCl}, 10 \mathrm{mM}$ HEPES, $2.7 \mathrm{mM} \mathrm{KCl}, 2 \mathrm{mM} \mathrm{MgCl}, 1 \%$ BSA in milliQ water (pH 7.45).

- Coverslip cleaning solution: $2 \mathrm{M} \mathrm{HCl}$ in $50 \%$ ethanol.

- $\mathrm{DiOC}_{6}$ in $1 \%$ DMSO in buffer

- Flow buffer: $136 \mathrm{mM} \mathrm{NaCl}, 10 \mathrm{mM}$ HEPES, $2.7 \mathrm{mM} \mathrm{KCl}, 2 \mathrm{mM} \mathrm{MgCl}, 2 \mathrm{mM} \mathrm{CaCl}, 0.1 \%$ glucose, $0.1 \% \mathrm{BSA}, 1 \mathrm{U} / \mathrm{mL}$ heparin in milliQ water (pH 7.45 with $\mathrm{NaOH})$.

- Platelet-adhesive substances for coating are described elsewhere. ${ }^{9}$ For coating collagen peptides, also see previous papers. ${ }^{11,12}$ Note that collagens and collagen solutions require storage in acid milieu. ${ }^{8,12}$

- $\quad$ PPACK in $10 \mathrm{mM} \mathrm{HCl}$

- Saline: $0.9 \% \mathrm{NaCl}$ in milliQ water (sterilized).

\section{Materials \& equipment}

- Glass coverslips ( 24 x $60 \mathrm{~mm}$, thickness $0.18 \mathrm{~mm}$ ) (Menzel BB024060A1)

- Precision mall with template for one or two rows of three microspots (3 mm centre-tocentre distance) for placement on glass coverslip. Note: apply either $1 \times 3$ or $2 \times 3$ microspots, depending on the possibility to observe these by the microscope.

- Humid chamber for storing coated coverslips.

- Open parallel-plate flow chamber: transparent polycarbonate block with engraved flow channel ( $50 \mathrm{~mm}$ depth, $3 \mathrm{~mm}$ width, $30 \mathrm{~cm}$ length, inlet/outlet tubes at an angle of $11^{\circ}$ ). The Maastricht chamber has been described before. It needs to be fixed in an aluminium holder with screws. ${ }^{4}$

- Silicon tubing (0.28 mm ID, $0.61 \mathrm{~mm}$ OD; Rubber BV Hilversum).

- Blunt syringe needles (18 gauge) to connect with tubing.

- Surgical tweezers to clamp tubing.

- Pulse-free syringe perfusion pump Type 100 (Harvard Instruments).

- Needle or system for blood drawing (e.g., 23 gauge).

- Plastic syringes $1 \mathrm{~mL}$ (Becton-Dickinson).

- $5 \mathrm{~mL}$ polystyrene tube (Greiner Bio-One). 


\section{A1. Preparation of coverslips}

CRITICAL: Wash cleaned coverslips thoroughly with water.

Degrease new coverslips (use tweezers) with $2 \mathrm{M} \mathrm{HCl}$ in $50 \%$ ethanol.

1. Wash coverslips twice with milliQ water to remove residual $\mathrm{HCl}$.

2. Leave coverslips to dry on drying rack.

\section{A2. Coating of coverslips}

CRITICAL: Prevent drying of biological material on coverslip by storing in humid environment.

3. Prepare coating material (collagen, collagen peptide, decorin, fibrinogen, fibronectin, laminin, osteopontin, rhodocytin, thrombospondin, vitronectin, VWF), at 50-250 $\mathrm{mg} / \mathrm{mL}$, as described. ${ }^{9}$

4. Mount coverslip onto precision mall for coating.

5. Apply $0.5 \mu \mathrm{L}$ of coating solution(s) in the assigned place(s), and remove from the mall.

6. Store coverslip in humid environment to prevent drying out of microspots. Allow coating material to bind for 60 minutes at room temperature.

7. Block uncoated glass with blocking buffer, and leave in humid environment for 30 minutes.

8. Wash blocked coverslip with saline. If not immediately used, leave coverslip in humid environment to prevent drying.

\section{A3. Assembly of coverslip and flow chamber}

CRITICAL: Check for leakage of the mounted flow chamber. Also check rigorously for absence of air bubbles before starting the experiment. Note that temperature changes can lead to appearance of air bubbles. Keep inlet tubing as short as possible.

9. Connect tubing to inlet and outlet of the flow chamber.

10. Rinse chamber and tubing with flow buffer, check for absence of air bubbles, and mount coated coverslip on top of chamber.

11. Place chamber with coverslip in aluminium holder and tighten screws.

12. Check that flow chamber system is leak-tight by perfusion with flow buffer, flush out any bubbles in chamber.

\section{A4. Drawing of human blood by venipuncture}

CRITICAL: Before drawing human blood, obtain permission from your Medical Ethical Committee, according to the local and national regulations, and get full informed consent from donor. Coagulation (traces of thrombin) need to be rigorously prevented by drawing without constraints, mixing well with anticoagulant, and incubation at $37^{\circ} \mathrm{C}$. Note that PPACK is only shortly active as an anticoagulant at neutral $\mathrm{pH}$. Other possible errors are described elsewhere. ${ }^{4}$

13. Add $0.5 \mathrm{~mL}$ saline into a $5 \mathrm{~mL}$ polystyrene blood collection tube. Add $40 \mathrm{U} / \mathrm{mL}$ (f.c.) fragmin, and add $40 \mu \mathrm{M}$ PPACK (f.c.) just before venipuncture.

14. Draw blood according to local protocols, and let smoothly flow into collection tube. Directly mix blood with anticoagulant solution. We prefer an open system using a 23 gauge needle, to ensure undisturbed flow. Discard first $1 \mathrm{~mL}$ of blood before filling blood collection tube.

15. Incubate the collected blood at $37^{\circ} \mathrm{C}$ for $10-15$ minutes to allow platelets to resensitize.

16. Preferably, determine platelet and red cell counts. A decrease in platelet count points to aggregation of platelets, e.g. by traces of thrombin.

17. Add additional $20 \mu \mathrm{M}$ PPACK (f.c.) once per hour. 


\section{A5. Blood perfusion through flow chamber}

CRITICAL: Check for correct pump settings to obtain the requested shear rate. Check for absence of air bubbles and fibrin clots during the whole experiment. Preferably use an inverted microscope. Several possible errors are described elsewhere. ${ }^{4}$

18. Only for experiments to determine stable platelet adhesion or thrombus volume, add 0.5 $\mu \mathrm{g} / \mathrm{mL} \mathrm{DiOC}{ }_{6}$ (f.c.) to $0.5 \mathrm{~mL}$ blood sample. Allow staining of the blood cells for 5 minutes.

19. Draw $0.5 \mathrm{~mL}$ blood sample into $1 \mathrm{~mL}$ syringe equipped with blunt needle. Make sure that no air is present in top of needle.

20. Connect syringe with blood sample to inlet tubing of prepared flow chamber. Carefully avoid air bubbles (fluid-fluid contact).

21. Prepare $1 \mathrm{~mL}$ syringe with flow buffer with required fluorescent labels. The following labels are suitable for post-staining: FITC-labeled anti-CD62P mAb $(1.25 \mu \mathrm{g} / \mathrm{mL})$ or FITC-labeled anti-fibrinogen mAb (1:100) and/or AF647-labeled annexin A5 (0.25 $\mu \mathrm{g} /$ $\mathrm{mL}$ ) (all f.c.).

22. Mount flow chamber and holder on stage of the microscope, and identify position of microspots with camera. Focus on optical plane of one microspot.

23. Place syringe filled with whole blood on perfusion pump (push mode). Ensure proper pump settings (arterial wall shear rate: $1600 \mathrm{~s}^{-1}$ for 3.5 minutes or $1000 \mathrm{~s}^{-1}$ for 4 minutes; venous wall shear rate $150 \mathrm{~s}^{-1}$ for 6 minutes). Note that the wall shear rate depends on the flow rate and the dimensions of the flow chamber. For calculation, see elsewhere. ${ }^{4}$

24. Switch pump on. The experiment starts when the blood has reached the site of the mi crospots.

\section{A6. Recording of stable adhesion and thrombus volume using DiOC $_{6}$-labeled platelets} CRITICAL: Rinse shortly to prevent disaggregation of thrombi.

25. During the first 2 minutes of whole blood perfusion, record $\mathrm{DiOC}_{6}$ fluorescence microscopic images at 2-seconds intervals (real-time recording of stable platelet adhesion).

26. After 3.5, 4 or 6 minutes of perfusion (depending on shear rate), change syringe with blood by syringe with flow buffer; set pump rate at $1000 \mathrm{~s}^{-1}$.

27. For 2 minutes, rinse chamber with flow buffer.

28. Take confocal $z$-stacks from thrombi during stasis; 2 to 3 stacks per microspot (recording of thrombus volume).

\section{A7. Recording of brightfield images and post-staining with fluorescent labels}

CRITICAL: Rinse shortly to prevent disaggregation of thrombi. Prevent air bubbles in flow chamber during syringe replacements.

29. Starting from point 25.

30. At the end of the whole-blood perfusion, change syringe with blood by syringe with fluorescent labels; set pump rate at $1000 \mathrm{~s}^{-1}$.

31. For 2 minutes, perfuse buffer with labels through flow chamber; leave 1 minute for staining.

32. During the perfusion, take brightfield microscopic images (5 per microspot) under flow.

33. Change syringe with labels by syringe with flow buffer; and perfuse for 2 minutes to remove unbound label.

34. Take fluorescence images during stasis (5 images per microspot). 


\section{Procedures B. Brightfield and fluorescence microscopic imaging of thrombi}

\section{B1. Use of LSM 7 LIVE line-scanning confocal fluorescence microscope}

SPECIFICATION: Recording of stable platelet adhesion and thrombus volume (DiOC -labeled platelets). Use in confocal mode for rapid real-time scanning of platelet adhesion, and of z-stacks to determine thrombus volume (see also Ref. ${ }^{12}$ ). Collect only sharp, high-quality images! Three color staining is possible (excitations 485, 530, $640 \mathrm{~nm}$ ).

1. Microscope: inverted confocal fluorescence microscope: Axio Observer Z1 (Carl Zeiss) with differential interference contrast (DIC) optics. Camera: AxioCam HRm (Zeiss). Scanning stage with insert for flow chamber holder.

2. Laser head: LSM 7 Live (Zeiss). Lasers: DOPP 488 nm (100 mW), DPSS 532 nm (75 $\mathrm{mW})$, Laser $635 \mathrm{~nm}(30 \mathrm{~mW})$.

3. Objective: $63 x$ oil immersion (Zeiss, PlanApo, NA 1.40; DIC M27, WD 0.19 mm).

4. Settings: [configuration 488 laser line]

a. Excitation $488 \mathrm{~nm}$, emission filter $495-555 \mathrm{~nm}$, pinhole $1 \mathrm{AU}$.

b. For time series: 1 cycle of 2 minutes with 2-seconds interval, laser power $5 \%$, gain 5 , zoom $0.5 x$, scan speed 3-4 Lps.

c. For z-stack: $0.5 \mu \mathrm{m}$ between optical planes (70 slices), laser power $5 \%$, gain 5 , zoom $1 \mathrm{x}$, scan speed 1 Lps.

5. Controlling software: ZEN 2010 (Zeiss).

6. Output images: LSM file ( $512 \times 512$ pixels, $107 \times 107 \mu \mathrm{m}$ or $213 \times 213 \mu \mathrm{m}$ (depending on zoom), 8-bit).

\section{B2. Use of BioRad/Zeiss Radiance $\mathbf{2 1 0 0}$ laser scanning confocal microscope}

SPECIFICATION: Imaging of thrombi post-labeled with FITC (OG488) and AF647 probes. Flow chamber with labeled thrombi is placed on stage up-side down. Scan with large pin holes to collect fluorescence from all optical planes. Collect only sharp, high-quality images! Three color staining is possible (excitations 485, 530, $640 \mathrm{~nm}$ ).

7. Microscope: right-up fluorescence microscope E600FN (Nikon, Japan). Scanning stage with insert for flow chamber holder.

8. Laser head: BioRad/Zeiss scan head. Lasers: Argon 488 nm (40 mW), Green He/Ne 543 $\mathrm{nm}(1.5 \mathrm{~mW})$, Red diode $638 \mathrm{~nm}(5 \mathrm{~mW})$.

9. Objective: $60 x$ oil immersion (Nikon, PlanApo SFluor, NA 1.30, WD $0.22 \mathrm{~mm}$ ).

10. Settings: Two-color fluorescence:

a. PMT1: excitation $488 \mathrm{~nm}$, laser power $20 \%$, iris 1.5 , emission filter $508-523 \mathrm{~nm}$

b. PMT2: excitation $637 \mathrm{~nm}$, laser power $50 \%$, iris 3.5, emission filter $>660 \mathrm{~nm}$

c. zoom 1, Kalman averaging 2, scan speed 160 Lps.

11. Recording software: LaserSharp 2000 software (Carl Zeiss).

12. Output images: PIC file (512 x 512 pixels, $200 \times 200 \mu \mathrm{m}, 8$-bit).

\section{B3. Use of camera-based non-confocal fluorescence microscope system}

SPECIFICATION: Imaging of thrombi post-labeled with FITC (OG488) and AF647 probes. Furthermore, recording of brightfield phase-contrast images to determine platelet deposition. Collect only sharp, high-quality images!

13. Microscope: inverted fluorescence microscope Diaphot 200 (Nikon) with phasecontrast. Two cameras connected with beam splitter, post-magnification and removable infrared filter. Vista brightfield CCD camera; Hamamatsu EM-CCD C9100-12 fluorescence camera. Scanning stage with insert for flow chamber holder.

14. Fluorescence: Xenon lamp (100 W). Filter cube: FITC (OG488): exciter $485 \pm 11 \mathrm{~nm}$, dichroic $400 \mathrm{~nm}$, emitter $530 \pm 15 \mathrm{~nm}$. Brightfield trans-illumination (white light). 
15. Objective: 40x oil-immersion (Nikon, Fluor/100, NA 1.30. Ph4DL, WD 0.20 mm).

16. Settings:

a. Brightfield phase-contrast (empty filter cube). Post-magnification: $1 x$

b. Fluorescence: excitation $485 \mathrm{~nm}$, emission $530 \mathrm{~nm}$. Post-magnification: 1.5x.

17. Recording software: Axiovision 4.8 (Zeiss).

18. Output images: TIFF file ( 512 × 512 pixels, $200 \times 200 \mu \mathrm{m}, 8-12$ bit).

\section{B4. Use of EVOS table fluorescence microscope}

SPECIFICATION: Imaging of thrombi post-labeled with FITC (OG488) and AF647 probes. Furthermore, recording of brightfield images to determine platelet deposition (overlays can be made). Collect only sharp, high-quality images! Three color staining is possible (excitations 485, 530, 640 $n m)$.

19. Microscope: EVOS-FL, inverted microscope, infinity-corrected fluorescence optical system.

20. LED diodes: DAPI $357 \mathrm{~nm}$ (emission $447 \mathrm{~nm}$ ), GFP $470 \mathrm{~nm}$ (emission $510 \mathrm{~nm}$ ), RFP 531 nm (emission 593 nm), Cy5 626 nm (emission 692 nm). Brightfield trans-illumination (white light).

21. Objective: $60 x$ oil immersion (Olympus, UPlanSApo, NA 1.35, WD $0.15 \mathrm{~mm}$ ).

22. Settings: adjustable intensity of LEDs:

a. Brightfield: transmitted light at intensity of $50 \%$.

b. GFP cube: excitation $470 \mathrm{~nm}$, emission $510 \mathrm{~nm}$, intensity $40 \%$.

c. Cy5 cube: excitation $626 \mathrm{~nm}$, emission $692 \mathrm{~nm}$, intensity $20 \%$.

23. Recording software: integrated in EVOS system. Make sure to save images of individual colors.

24. Output images: TIFF file (1360 x 1024 pixels, $142 \times 107 \mu \mathrm{m}, 8$-bit).

\section{Procedures C. Analysis of brightfield and fluorescence images}

\section{C1. Image analysis for morphological score}

CRITICAL: Analysis of images blinded for the experimental condition.

1. Determine morphological score of thrombi on coverslip based on recorded brightfield phase-contrast or DIC images.

2. Score at a 5-point scale (see Figure 1)

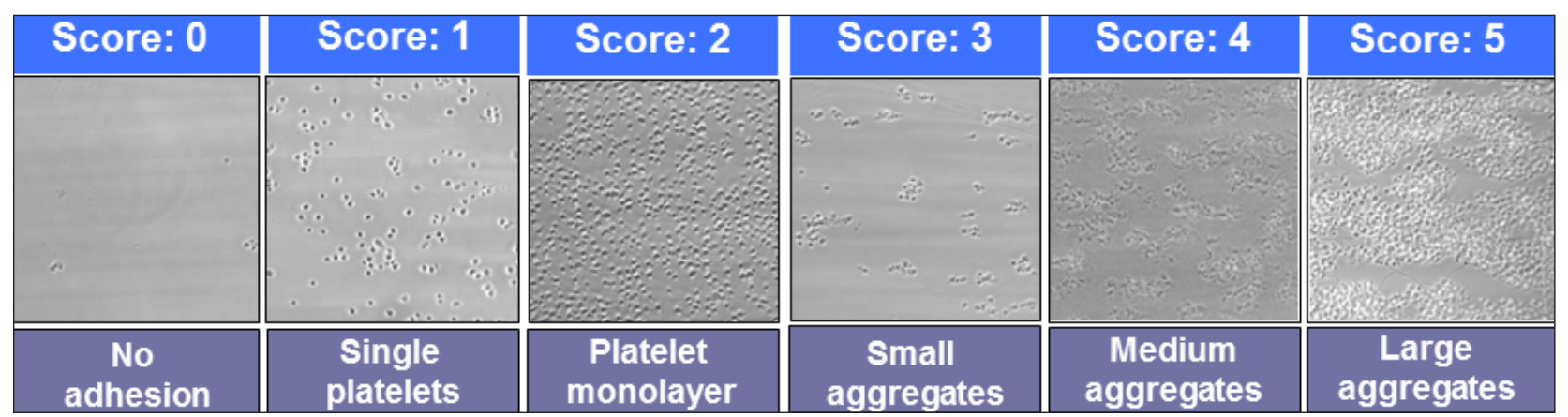

Figure 1. Assessment of morphological score of thrombi on 0-5 point scale. Representative images are given with description of scores. 


\section{C2. Image analysis with package Metamorph (Molecular Devices)}

CRITICAL: Measurement of surface area coverage of brightfield and fluorescence images.

The following procedures apply to 8-bit TIFF and PIC images. Conversion to 8-bit images can be done using ImageJ software (Open access). Image analysis can also be performed with ImageJ. Output data are given as numbers of pixels per region. To determine surface area coverage, use total pixel number of images. Note that the outcome of the analyses depends on the quality of the recorded images.

\section{Protocol for stable platelet adhesion (see Figure 2)}

a. Threshold every image within one time series with the same threshold $>$ binary image.

b. In "process" and "arithmic", choose the first binary image as "source image 1" and the second binary image as "source image 2".

c. Click "subtract" with constant values at " 0 ".

d. Choose apply.

e. Repeat steps $a-d$ for the next images.

f. Choose "measure" > "integrated morphometry analysis". Measure all binary im ages and subtracted images.

g. Export all values to Excel spreadsheet, and calculate \% of change between con secutive images.

\section{Protocol for surface area coverage of aggregated platelets (see Figure 3A)}

a. For each image, use edge detection in both horizontal (150) and vertical (150) direction.

b. Use the horizontally filtered image for threshold setting $>$ binary image.

c. Use morphological close filter (diamond, width $=12$ ) and open filter (circle, diameter $=5$ ).

d. Transfer regions to brightfield/fluorescence image, and check if region detection is right.

e. Export data to Excel file, and convert pixel numbers to \% surface-area-coverage.
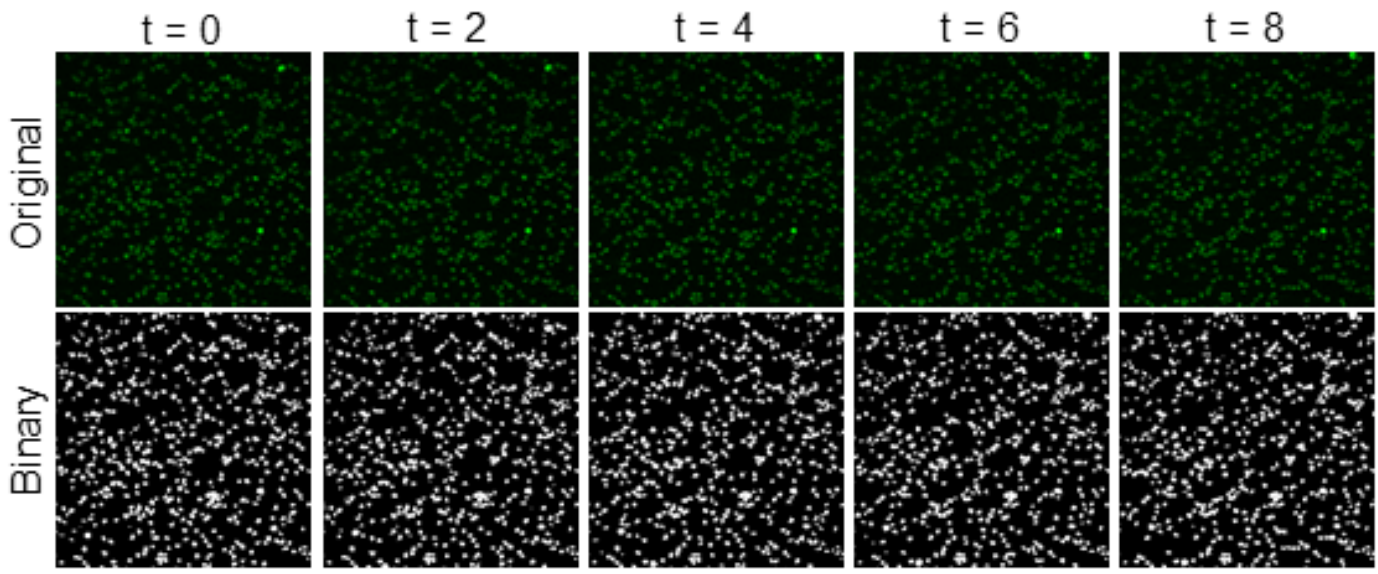

0
$\frac{1}{0}$
$\frac{0}{60}$
$\frac{6}{5}$
$\frac{0}{7}$
0
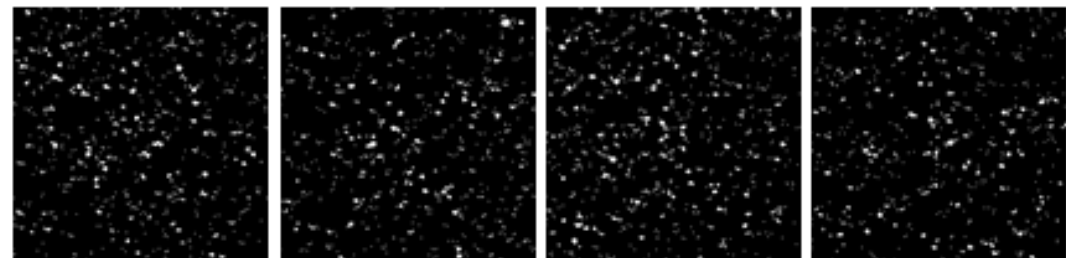

Figure 2. Intermediate processed images for determination of stable platelet adhesion, as described in the protocol. Time is in seconds. 


\section{Protocol for surface area coverage of platelet monolayers (see Figure 3B)}

a. Filter images using morphological bottom hat filter (diamond, width $=15$ ), then close filter (diamond, width $=4$ ).

b. Threshold closed image $>$ binary image.

c. Apply morphological dilate filter (square, width $=2$ ).

d. Transfer regions to original image, and check if region detection is right.

e. Export data to Excel file, and convert pixel numbers to \% surface area coverage.

\section{Protocol for integrated feature size}

a. The integrated feature size (IFS) is a value taking into account the proportional contribution of large and small thrombi on microspots. It represents the cumulative contribution of squared features, ranked from small to large individual features, with $(f)$ from small to large are numbered $1-N$. The IFS is calculated as:

$$
\sum_{i=1}^{N}\left(f_{i}^{2}\right) / \sum_{i=1}^{N} f_{i}
$$

b. First, from an analyzed image, rank the individual features (pixels per region) from small to large (one image $=$ one column with features) in an Excel file.

c. Determine the pixel size of one single platelet. Exclude all features smaller than this size ( $\approx 100$ pixels).

d. Integrate the values of the features (accumulated sum of pixels).

e. Convert pixel size into $\mu \mathrm{m}^{2}$.

f. Divide the accumulated feature size by the accumulated sum of all feature sizes, and express as percentage.

g. Calculate the area above the percentage curve in $\mu \mathrm{m}^{2}$.

h. Express results on a logarithmic scale.
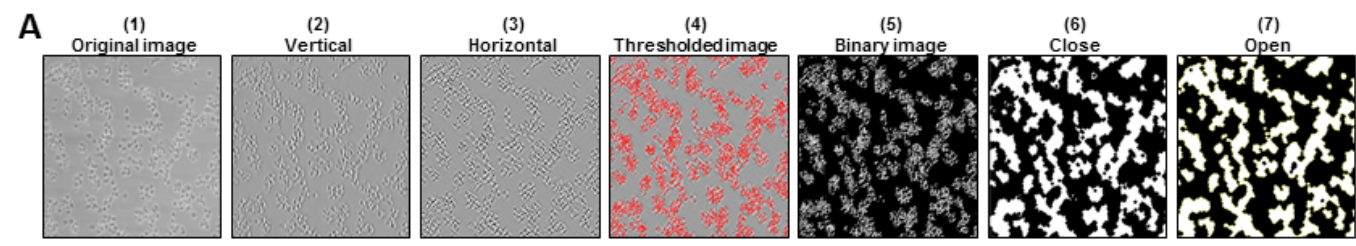

(8)
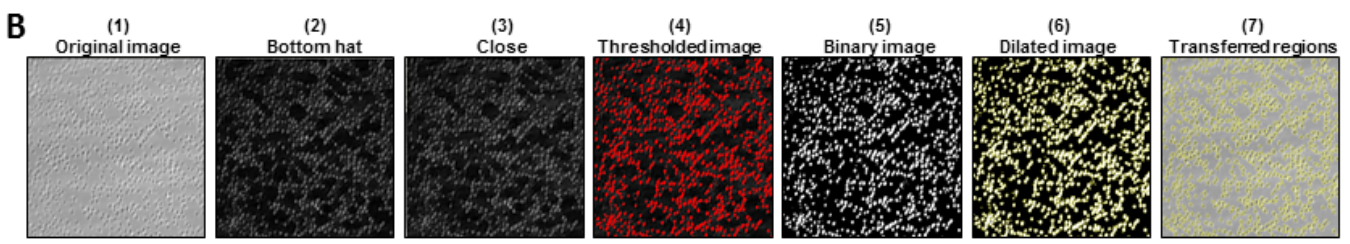

C

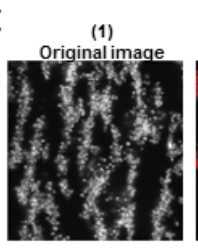

(2)
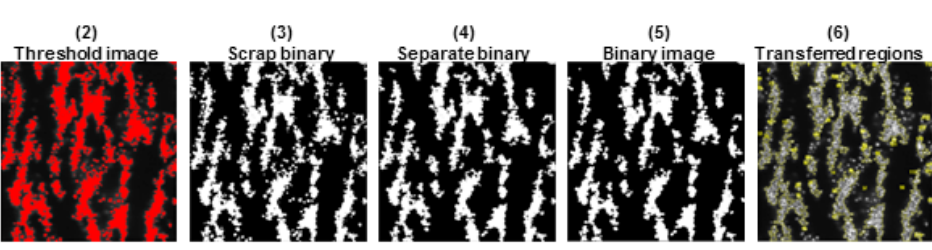

Figure 3. Results of automated image analysis, as described in the protocols. Sequences to determine surface area coverage of aggregated platelets (A) and of platelet monolayers (B), starting from phase-contrast images. Sequence to determine thrombus volume from stacks of fluorescence images (C). 


\section{C3. Image analysis with package Axiovision 4.8 (Zeiss) for thrombus volume}

CRITICAL: This program uses LSM files, and allows writing of scripts for automated image analysis. Common output is: ID region, volume unscaled (pixe/ $)^{\beta}$, surface $\left(\mu m^{2}\right)$ and volume $\left(\mu m^{3}\right)$ per region. Summative data can be calculated per region.

1. Use scrap filter with minArea: 1 and maxArea: 100 (see Figure 3C).

2. Use separation filter with count: 3 and in Morphology mode.

3. Transfer regions to original image, and check if region detection is right.

4. Export data to Excel file, and convert pixel numbers to $\mu \mathrm{m}^{3}$.

\section{References}

1. Swieringa F, Kuijpers MJ, Heemskerk JW and van der Meijden PE. Targeting platelet receptor function in thrombus formation: the risk of bleeding. Blood Rev 28, 9-21 (2014).

2. Roest M, Reininger A, Zwaginga JJ, King MR and Heemskerk JW. Flow chamber-based assays to measure thrombus formation in vitro: requirements for standardization. $J$ Thromb Haemost 9, 232223224 (2011).

3. Cosemans JM, Mattheij NJ, Angelillo-Scherrer A and Heemskerk JW. The effects of arterial flow on platelet activation, thrombus growth and stabilization. Cardiovasc. Res. 99,342-352 (2013).

4. Van Kruchten R, Cosemans JM and Heemskerk JW. Measurement of whole blood thrombus formation using parallel-plate flow chambers: a practical guide. Platelets 23, 229-242 (2012).

5. Versteeg HH, Heemskerk JW, Levi M and Reitsma PS. New fundamentals in hemostasis. Physiol Rev 93, 327-358 (2013).

6. Ruggeri ZM and Mendolicchio GL. Adhesion mechanisms in platelet function. Circ Res 100,1673-1685 (2007).

7. Siljander PR, Munnix IC, Smethurst PA, Deckmyn H, Lindhout T, Ouwehand WH, Farndale RW and Heemskerk JW. Platelet receptor interplay regulates collagen-induced thrombus formation in flowing human blood. Blood 103,1333-1341 (2004).

8. Heemskerk JW, Sakariassen KS, Zwaginga JJ, Brass LF, Jackson SP, Farndale Collagen surfaces to measure thrombus formation under flow: possibilities for standardization. $J$ Thromb Haemost 9,856858 (2011).

9. De Witt SM, Swieringa F, Cavill R, Lamers MM, van Kruchten R, Mastenbroek T, Baaten C, Coort S, Pugh N, Schulz A, Scharrer I, Jurk K, Zieger B, Clemetson KJ, Farndale RW, Heemskerk JW and Cosemans JM. Identification of platelet function defects by multi-parameter assessment of thrombus formation. Nat Commun 5, 4257 (2014)

10. Heemskerk JW, Mattheij $\mathrm{N}$ and Cosemans JM. Platelet-based coagulation: different populations, different functions. J Thromb Haemost 11, 2-11 (2013).

11. Munnix IC, Gilio K, Siljander PR, Raynal N, Hackeng T, Feijge MA, Deckmyn H, Farndale RW and Heemskerk JW. Affinity modulation of triple-helical peptides for binding to integrin $a_{2} \beta_{1}$ and glycoprotein VI affects thrombus formation under flow: a study with collagen-mimicking peptides. $J$ Thromb Haemost 7, 2132-2142 (2008).

12. Pugh N, Simpson AM, Smethurst PA, de Groot PG, Raynal N and Farndale RW. Synergism between platelet collagen receptors defined using receptor-specific collagen-mimetic peptide substrata in flowing blood. Blood 115, 5069-5079 (2010).

13. Pugh N, Bihan D, Perry DJ and Farndale RW. Dynamic analysis of platelet deposition to resolve platelet adhesion receptor activity in whole blood at arterial shear rate. Platelets, in press (2014). 


\section{CHAPTER 4}

IDENTIFICATION OF PLATELET

FUNCTION DEFECTS BY

MULTI-PARAMETER ASSESSMENT

OF THROMBUS FORMATION

De Witt SM, Swieringa F, Cavill R, Lamers MM, van Kruchten R, Mastenbroek T, Baaten C, Coort S, Pugh N, Schulz A, Scharrer I, Jurk K, Zieger B, Clemetson KJ, Farndale RW, Heemskerk JW, Cosemans JM 


\begin{abstract}
Assays measuring platelet aggregation (thrombus formation) at arterial shear rate mostly use collagen as only platelet-adhesive surface. Here we report about a multi-surface and multi-parameter flow assay to characterize thrombus formation in whole blood from healthy subjects and patients with platelet function deficiencies. A systematic comparison is made of 52 adhesive surfaces with components activating the main platelet adhesive receptors, and of 8 output parameters reflecting distinct stages of thrombus formation. Three types of thrombus formation can be identified with a predicted hierarchy of the following receptors: glycoprotein (GP)VI, C-type lectin-like receptor-2 $\left(\right.$ CLEC-2) $>$ GPIb $>a_{6} \beta_{1}, a_{\text {IIb }} \beta_{3}>a_{2} \beta_{1}>$ CD36, $a_{5} \beta_{1}, a_{v} \beta_{3}$. Application with patient blood reveals distinct abnormalities in thrombus formation in patients of severe combined immune deficiency, Glanzmann's thrombasthenia, Hermansky-Pudlak syndrome, May-Hegglin anomaly or gray platelet syndrome. We suggest this test may be useful for the diagnosis of patients with suspected bleeding disorders or a pro-thrombotic tendency.
\end{abstract}

\title{
Introduction
}

The fundamental role of platelets in hemostasis and thrombosis relies on their capability of adhesion to specific locations of the perturbed vessel wall upon injury, damage or inflammation. Continued adhesion of flowing platelets leads to buildup of a platelet plug or thrombus and is required to stop bleeding or, under pathological conditions, to induce thrombosis, for instance after rupture 0 an atherosclerotic plaque ${ }^{1}$. Many experimental studies with genetically modified mice or with blood from patients with hemostatic deficiencies, performed at either arterial (high shear rate) or venous (low shear rate) flow conditions, have emphasized that thrombus formation is a complex process encompassing multiple platelet receptors and signaling mechanisms ${ }^{2-4}$.

For over two decades, parallel-plate flow chambers have been used to measure platelet adhesion and activation under arterial or venous flow conditions, in particular using surfaces such as extracellular matrix or collagen ${ }^{5,6}$. Currently, this process of platelet adhesion and aggregation in flow devices is described as flow-dependent thrombus formation, regardless of the presence or absence of anticoagulants. ${ }^{4}$ Whole blood flow chamber tests with blood from many strains of genetically modified mice have revealed platelet function defects under flow in vitro that often associate with a reduced arterial thrombosis tendency in vivo ${ }^{1,7,8}$. These outcomes have boosted the use of commercial and home-made flow devices, in particular for the assessment of human platelet activity in preclinical settings, in spite of the fact that international recommendations stress the need for further standardization of devices, protocols and measurement parameters ${ }^{9}$. In recent years, various types of microfluidic devices have been developed requiring only small volumes of human blood ${ }^{10,11}$, including devices containing endothelium, for instance to study blood from patients with sickle cell disease ${ }^{12}$. Unfortunately however, the great variation in design and use of the microfluidic chips hinders the process of standardization ${ }^{13}$. On the other hand, relatively simple, one-parameter microfluidics tests using collagen surfaces have already been employed to determine inter-subject variability and the efficacy of antiplatelet therapy in cardiac patients ${ }^{14-17}$.

Clinically, the PFA-100 is the only device currently validated that assesses platelet function under high-shear flow conditions, by measuring the occlusion time due to platelet aggregation on a collagen matrix. The PFA-100 is frequently utilized to evaluate deficiencies in platelet function or von Willebrand factor (VWF) activity, but it only provides a single end-stage parameter. Current guidelines for laboratory investigations to check for heritable disorders of platelet function recommend the PFA-100 as an optional screening test, but also stipulate that this test is not diagnostic and is insensitive to mild platelet disorders ${ }^{18}$. Taken together, there are promising possibilities for clinical employment of flow assays to test platelet adhesion and aggregation, but current methods often are incompletely developed and insufficiently standardized.

The classic concept of flow-dependent thrombus formation is based on collagen-dependent models of platelet aggregation, both in vivo in damaged mouse vessels and in vitro using 
collagen-coated flow devices. Fibrillar collagen (collagen I or III) is considered as the primary platelet-activating substance in the damaged vessel wall controlling the thrombotic process $24,8,19,20$. The concept, in brief, is that at high arterial wall shear rate, initial platelet rolling is regulated by the interaction of platelet glycoprotein Ib-V-IX (GPIb) to VWF which is bound to collagen. Platelet adhesion and activation by VWF/collagen then is enforced by interplay of the collagen receptors, glycoprotein VI (GPVI) and integrin $a_{2} \beta_{1}$, and the fibrinogen receptor, integrin $a_{\mathrm{IIb}} \beta_{3}^{21,22}$. Platelets may first adhere via integrins and then become activated via GPVI, or first interact with GPVI ${ }^{23}$. Collagen-induced activation of platelets includes rises in cytosolic $\mathrm{Ca}^{2+}$, secretion of dense and a-granules (monitored as P-selectin expression) and release of autacoids such as ADP, ATP and thromboxane $A_{2}$, thus resulting in a plethora of paracrine substances able of recruiting and capture other passing platelets. Together with GPVI, these mediators induce affinity changes in integrins $a_{2} \beta_{1}$ and $a_{\text {IIb }} \beta_{3}$, which are required for thrombus stability ${ }^{24,25}$. Additionally, GPVI signaling stimulates platelet procoagulant activity and thrombin generation via phosphatidylserine exposure ${ }^{26}$.

However, in the perturbed vessel wall, platelets will be in contact with many other adhesive ligands than only collagen and VWF. Platelets indeed express adhesive receptors for a large number of vascular and plasma proteins ${ }^{2}$. So far, adhesion of platelets under flow conditions has been studied on surfaces coated with fibrinogen ${ }^{27}$, fibronectin ${ }^{28}$, vitronectin ${ }^{29,30}$, osteopontin, laminin ${ }^{31,32}$, and thrombospondin-133. Another relevant receptor is the C-type lectin-like receptor-2 (CLEC-2), which still lacks a clear physiological ligand, but supports in murine studies thrombus formation in a similar way to the collagen receptor, GPVI ${ }^{34-36}$. Precisely how these adhesive proteins and receptors support thrombus formation under flow conditions in comparison to collagen and GPVI remains poorly understood.

In this paper, we show a first systematic study to compare key physiological platelet-adhesive proteins for all major adhesive receptors, alone and in combination, to support whole blood thrombus formation at specified wall shear rates. Based on this inventory, we developed a multi-microspot test using nine different surfaces, which is validated using blood samples from patients with distinct platelet function deficiencies. Using systems biology approaches, we use the test outcomes for a model predicting the roles of various receptors in thrombus formation at high and low wall-shear rate, and for a template determining aberrations in this process in patients with platelet dysfunctions.

\section{Materials and Methods}

\section{Materials}

Sources of proteins for microspot coatings are indicated in Suppl. Table 5. VWF purified from human plasma was obtained from University Medical Center Utrecht ${ }^{45}$. Rhodocytin was purified by gel filtration and ion exchange chromatography from venom of the Malayan pit viper, Calloselasma rhodostoma ${ }^{46}$. The following triple-helical peptides were synthesized as C-terminal amides and were purified by reverse phase high performance liquid chromatography ${ }^{47}: \mathrm{H}-\mathrm{GPC}(\mathrm{GPO})_{3} \mathrm{GFO}-$ GERGPO) ${ }_{3}$ GPC-NH ${ }_{2}$ [GFOGER-(GPO) $)_{n} ; \mathrm{H}-\mathrm{GPC}(\mathrm{GPP})_{5}$ GFOGER(GPP) ${ }_{5}$ GPC-NH ${ }_{2}$ [GFOGER-(GPP) ${ }_{n}$ ] and cross-linked collagen-related peptide, (GPO) ${ }_{\mathrm{n}}$ (Ref. 48); H-GPC(GPP) $\left.{ }_{5} \mathrm{GPRGQOGVMGFO(GPP)}\right)_{5} \mathrm{G}-$ PC-NH ${ }_{2}$, collagen type-III derived VWF-binding peptide (VWF-BP), also called VWF-III (Ref. 44). BSA was obtained from Sigma; $D$-phenylalanyl-L-prolyl-L-arginine chloromethylketone (PPACK) from Calbiochem; 3,3'dihexyloxacarbocyanine iodide $\left(\mathrm{DiOC}_{6}\right)$ from AnaSpec; corn trypsin inhibitor from Haematologic Technology Inc.; recombinant human tissue factor from Dade-Behring; GlyPro-Arg-Pro (GPRP) from Stago.

Anti-fibrinogen antibody labelled with fluorescein isothiocyanate (FITC) was from WAK Chemie; FITC-labelled anti-CD62P (P-selectin) mAb from Immunotech; annexin A5 labelled with Alexa fluor (AF)647 from Molecular Probes. Blocking mAbs against specific receptors came from the following sources: mAb-1950 against integrin $a_{2}$, mAb-1976 against integrin $a_{v} \beta_{3}$ and mAb-1969 
against $a_{5} \beta_{1}$ from Merck-Millipore; Fab-152 against CD36 from Santa Cruz; chimeric mAb abciximab, directed against integrins $a_{\text {IIb }} \beta_{3}$ and $a_{v} \beta_{3}$ from Centocor. Sources of single-chain $A b 10 B 12$ against GPVI, ${ }^{48} \mathrm{mAb}$ 15D7 against integrin $\mathrm{a}_{2} \beta_{1}$ (ref. 48) and of $6 \mathrm{~B} 4 \mathrm{Fab}_{2}$ fragment against the VWF-binding site on GPIba ${ }^{49}$.

\section{Microspotting of proteins and peptides}

Glass coverslips ( $24 \times 60 \mathrm{~mm}$, Menzel) were degreased with $2 \mathrm{M} \mathrm{HCl}$ in $50 \%$ ethanol, and rinsed with water and saline. Using a precision mall, arrays of three consecutive microspots ( $3 \mathrm{~mm}$ center-to-center distance) were applied on coverslips as $0.5 \mu \mathrm{L}$ volumes of coating proteins or peptides. Coating concentrations were optimized to give maximal static platelet adhesion, previously established in the range of $50-250 \mu \mathrm{g} / \mathrm{mL}$ (Ref. 24,37,45). Osteopontin, vitronectin and VWF were applied at $50 \mu \mathrm{g} / \mathrm{mL}$; collagens, VWF-BP, laminin and thrombospondin-1 at $100 \mu \mathrm{g} / \mathrm{mL}$; and other proteins and collagen-related peptides at $250 \mu \mathrm{g} / \mathrm{mL}$. Coated podoplanin did not support platelet adhesion, and was not used for further experiments. Sources of coated proteins and peptides are given in Suppl. Table 5. Microspots with double or triple coatings were prepared by mixing the desired proteins or peptides at the concentrations above.

Microspot-coated coverslips were incubated for $1 \mathrm{~h}$ in a humid atmosphere, and washed twice with saline. Coatings were verified by staining with Coomassie brilliant blue $\mathrm{G}$, showing circular stained spots of $\sim 1000 \mu \mathrm{m}$ in diameter for most coated proteins; only laminin and collagen I gave smaller $(800 \mu \mathrm{m})$ and larger $(1200 \mu \mathrm{m})$ spots, respectively. Before flow perfusion, coverslips were blocked with HEPES buffer pH 7.45 (136 mM NaCl, 10 mM HEPES, $2.7 \mathrm{mM} \mathrm{KCl}, 2 \mathrm{mM} \mathrm{MgCl}{ }_{2}$ $0.1 \%$ glucose, $1 \mathrm{U} / \mathrm{mL}$ heparin) supplemented with $1 \%$ BSA.

\section{Healthy control subjects and patients}

Studies were approved by the local Medical Ethics Committee (Maastricht University Medical Centre, NL31480.068.10). Blood samples $(10 \mathrm{~mL})$ were taken from healthy control subjects and patients, who had not used antiplatelet or anti-inflammatory medication for two weeks. All donors gave informed consent in accordance to the Declaration of Helsinki. Healthy controls (both sexes, age 23-58 years) were not under medical care, had not experienced bleeding problems, and had normal platelet counts $\left(200-300 \times 10^{\%} / \mathrm{l}\right)$ and haematocrit values $(32-42 \%)$. For determination of intra-subject variability, blood samples from six healthy donors were taken on four different days, perfused and analysed.

Patients all had a well-defined platelet deficiency and mild bleeding tendency, as reported by the examining physicians: a patient with Glanzmann's thrombasthenia with confirmed deficiency in platelet $\mathrm{a}_{\mathrm{IIb}} \beta_{3}$ expression and normal blood cell counts ${ }^{50}$; a patient with severe immunodeficiency syndrome (SCID), ORAI1 mutation and abolished store-regulated influx in platelets ${ }^{51}$; a patient with May-Hegglin anomaly (MYH9 gene mutation) displaying macrothrombocytopenia $\left(9 \times 10^{9}\right.$ platelets/L) and a relatively high hematocrit level (46\%); a patient with confirmed Hermansky-Pudlak syndrome (HPS3 gene mutation), characteristically lacking platelet dense granules, as confirmed by flow cytometry $\left(280 \times 10^{9}\right.$ platelets/L , hematocrit $\left.31 \%\right)$; a patient with suspected gray platelet syndrome with major deficiency in platelet a-granules and reduced P-selectin expression, which was accompanied by macrothrombocytopenia $\left(37 \times 10^{9}\right.$ platelets/L, hematocrit $\left.33 \%\right)$. Blood samples from every patient were run in parallel to blood samples from healthy travel controls.

\section{Blood collection}

Blood collection was into 0.1 volume of saline containing PPACK $(40 \mu \mathrm{M})$ and fragmin $(40 \mathrm{U} / \mathrm{mL}$, final concentrations); alternatively, where indicated, collection was into 0.1 volume of sterile 129 $\mathrm{mM}$ trisodium citrate. Citrated blood samples were recalcified with $3.75 \mathrm{mM} \mathrm{MgCl}$ and $7.5 \mathrm{mM}$ $\mathrm{CaCl}_{2}$ (final concentrations) in the presence of PPACK $(40 \mu \mathrm{M})$ and fragmin $(40 \mathrm{U} / \mathrm{mL})$ prior to the flow experiment. Prior to perfusion, blood samples were checked for platelet counts and the ab- 
sence of visible clots.

\section{Flow chamber device and flow perfusion protocol}

Microspot-coated coverslips were mounted onto a transparent parallel-plate flow chamber (50 $\mu \mathrm{m}$ depth, $3 \mathrm{~mm}$ width and $20 \mathrm{~mm}$ length), and pre-rinsed with HEPES buffer $\mathrm{pH} 7.45$ containing $0.1 \%$ BSA. Anticoagulated whole blood samples (400-500 $\mu \mathrm{L}$ ) were perfused through the flow chamber for a time period sufficient for full thrombus formation on collagen I spots, i.e., 6 min at $150 \mathrm{~s}^{-1}$, $4 \mathrm{~min}$ at $1000 \mathrm{~s}^{-1}$, and $3.5 \mathrm{~min}$ at $1600 \mathrm{~s}^{-1}$. Where indicated, blood samples were pre-incubated for 5 min with DiOC $_{6}(0.5 \mu \mathrm{g} / \mathrm{mL})$, and fluorescence images were recorded from the microspots during blood perfusion. In other cases, thrombi formed after blood flow were poststained by 2-min perfusion $\left(1000 \mathrm{~s}^{-1}\right)$ with color-selected combinations of the following platelet activation markers: FITC-labelled anti-fibrinogen mAb (1:100), FITC-labelled anti-P-selectin mAb $(1.25 \mu \mathrm{g} / \mathrm{mL})$ and/or AF647-annexin A5 $(0.25 \mu \mathrm{g} / \mathrm{mL})$, all in HEPES buffer $\mathrm{pH} 7.45$ supplemented with $0.1 \%$ BSA. After 2 min of staining (stasis), unbound label was removed by a short perfusion with the same HEPES buffer. No fixative was used.

To assess the role of thrombin on thrombus formation under high shear flow conditions, tissue factor (500 pM) was immobilized together with a dual coating of VWF with fibronectin or collagen I. Corn trypsin inhibitor (CTI, $5 \mu \mathrm{g} / \mathrm{mL}$ ) was present in the blood collecting tube to inhibit the contact activation pathway of coagulation. Blood was drawn on $0.32 \%$ trisodium citrate and Gly-Pro-Arg-Pro ( $5 \mathrm{mg} / \mathrm{mL}$ ) was added to block fibrin polymerization and thus clot formation. During the flow perfusion the blood was recalcified with $6.3 \mathrm{mM} \mathrm{CaCl}$ and $3.2 \mathrm{mM} \mathrm{MgCl}$ to allow thrombin to be formed via co-coated tissue factor. Platelet activation markers were determined as described for the noncoagulating conditions.

\section{Standardized recording of microscopic images}

Phase-contrast bright-field images were recorded from all three microspots during buffer perfusion immediately after outflow of the red blood cells, using an inverted Nikon Diaphot microscope, equipped with a $40 \times$ oil-immersion objective (N.A. 1.3), a $1.8 \times$ relay lens, and a CCD camera (640 $\times 480$ pixels) ${ }^{38}$. For measuring platelet activation markers, the flow chamber was placed on the stage of a confocal BioRad/Zeiss MRC-600 microscope, equipped with a $60 \times$ oil immersion objective (N.A. 1.4). Dual-color confocal fluorescence 8-bit images $(512 \times 512$ pixels) were recorded at 488 and $633 \mathrm{~nm}$ excitation and settings, as described previously ${ }^{52}$. Thrombus volume was determined using a fast line-scanning Zeiss LSM7 microscope system, equipped with a $63 \times$ oil objective. Confocal $z$-stacks were recorded of $\mathrm{DiOC}_{6}$-labelled platelets in thrombi (8-bit images of $512 \times 512$ pixels; $106 \times 106 \mu \mathrm{m}$, stack distance $0.5 \mu \mathrm{m})$. The same label and system was used to measure stable platelet adhesion during blood flow (8-bit images, taken at 2-s intervals). For each flow run, 5 representative microscopic images were taken from each of the microspots. Flow assays per blood sample were performed in duplicate or in triplicate, if duplicates showed marked variation.

\section{Quantitative analysis of recorded images}

Phase-contrast and fluorescence images were analysed by standardized journals, using Metamorph software (Molecular Devices), or in the case of LSM7 images with Axiovision Rel.4.8 software (Carl Zeiss). End-stage phase-contrast images of adhered platelets were judged as follows. The morphological score was obtained by visual inspection of the platelet features per microspot: 0 , no or hardly any adhered platelets; 1 , multiple single adhered platelets; 2 , extensive coverage of single adhered platelets; 3, small platelet aggregates; 4, intermediate platelet aggregates; 5, full thrombi with large-size platelet aggregates. Platelet deposition was determined as surface area coverage by using supervised image analysis journals. In brief, auto-enhanced images were filtered vertically and horizontally and thresholds were set. The resulting binary images were subjected to a close and open filter, which resulted in identified regions of single or clustered adhered platelets. The 
integrated feature size was determined as a parameter, taking into account a proportional contribution of large and small thrombi on microspots. It was defined as the cumulative contribution of squared feature areas $\left(f^{2}\right)$, ranked from small to large $(1-N)$, to the total feature size $(S f)$ (Equation 1):

$$
\sum_{i=1}^{N}\left(f_{i}^{2}\right) / \sum_{i=1}^{N} f_{i}
$$

In cumulative plots, where larger size features appear at the right, the area above the curve represents a value of the integrated feature size.

Time series of images of $\mathrm{DiOC}_{6}$ fluorescence (1 min, 2-s intervals) were converted into subtracted, differential images using Metamorph software, and analyzed for changes in pixel intensity above background ${ }^{53}$, thus producing a value for stable platelet adhesion. Confocal fluorescence images were thresholded with predefined journals using Metamorph software, to obtain percentage values of SAC for each platelet activation label: FITC-anti-fibrinogen mAb ( $a_{\text {IIb }} \beta_{3}$ activation), FITC-anti-P-selectin mAb (a-granule secretion; correction for non-specific labeling), AF647-annexin A5 (phosphatidylserine exposure) ${ }^{38}$. Z-stacks of confocal images of DiOC 6 fluorescence were analyzed with Axiovision software to obtain a summed thrombus volume per surface area ${ }^{37}$. Protocols were checked by three different observers, who were blinded to the experimental variables.

\section{Bioinformatics and statistics}

For comparative analysis, mean values of all thrombus parameters from 52 surfaces were linearly normalized to a range from 0-10 (values of all parameters were normally distributed). Two-way unsupervised hierarchical clustering was performed using the $\mathrm{R}$ package version 2.3 (www.r-project.org). Euclidean distances were calculated, and clustering was by complete linkages. Robustness of the clusters was checked using the R program, Pvclust. The clusters were rebuilt based on 10,000 randomizations, and their significance was assessed using an approximately unbiased $P>90$. Pvclust was also used to evaluate the robustness of the clusters by leaving out particular surfaces or parameters, with the aim to select those surfaces and parameters giving non-redundant information. Partial least squares regression models with $\beta$-matrices were built in Matlab, and were employed to make 2-3 component prediction models for thrombus type and platelet receptor contribution. All models were checked by cross-validation predictions.

Parameters were correlated or compared by multiple regression analysis using the statistical package for social sciences (SPSS 19.0). Patient data were compared to normal ranges established for healthy control subjects, and statistically analyzed by probability analysis. Effects of antibodies and heatmap subtractions were compared using a two-tailed Student's t-test.

\section{Results}

\section{Arrays of adhesive surfaces to assess thrombus formation}

To compare the roles of established platelet-adhesive receptors in thrombus formation, we selected ligands of these receptors that are present in either the vessel wall or platelet aggregates. In addition, we used a number of chemically synthesized peptides binding to the same receptors (Suppl. Table 1). In preliminary experiments, we used different coating concentrations to assure that the selected proteins and peptides: (i) remained bound to degreased glass coverslips after repeated rinsing (Coomassie staining), and (ii) showed optimal platelet adhesion under static conditions (S.M.d.W., personal communication). Optimized surfaces for platelet adhesion consisted of the following proteins: collagens I and III, decorin, fibrinogen, fibronectin, laminin 511/521, osteopontin, thrombospondin-1, vitronectin and/or VWF (Figure 1). Also included were the snake venom rhodocytin, four collagen-mimetic triple-helical peptides, i.e. GFOGER-(GPP) ${ }_{n}$ GFOGER-(GPO) ${ }_{n^{\prime}}(\mathrm{GPO})_{n^{\prime}}$ and a VWF-binding peptide (VWF-BP). Immobilized bovine serum albumin (BSA) served 


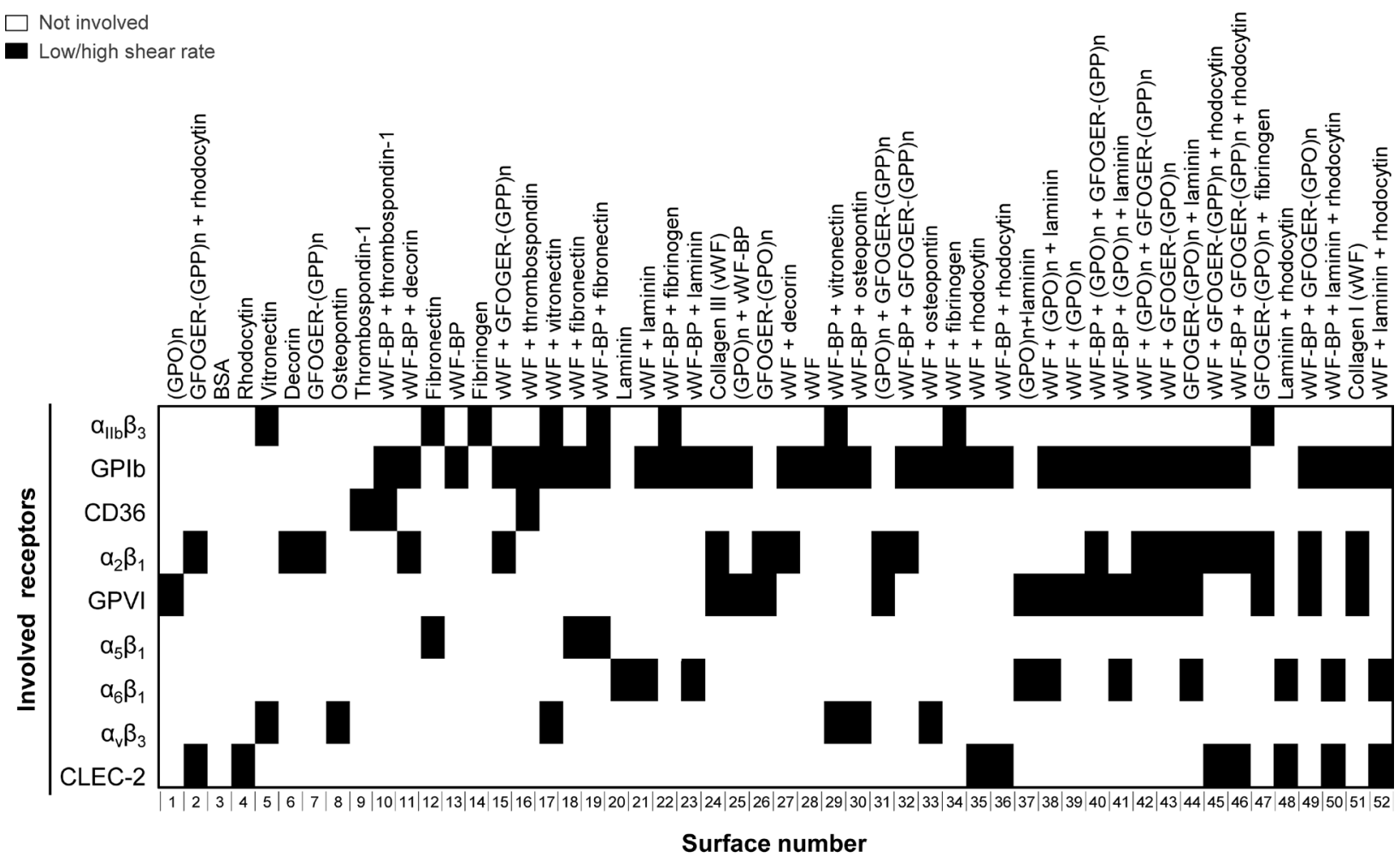

Figure 1. Protein surfaces used for flow studies and assignment map of platelet receptors interacting with indicated proteins. Assignment of interactions of platelet receptors to immobilized protein or peptide ligands was as represented in Suppl. Table 1. Surfaces were numbered 1-52, based on unsupervised hierarchical cluster analysis of thrombus parameters (see Figure 4). Color code: white, not involved; black, involved at low/high wall shear rate.

as negative control surface. Optimally effective coating concentrations of the collagen-mimetic peptides were established before ${ }^{24,37}$.

By coating these proteins/peptides alone or in combinations as a row of three microspots (diameter $1000 \mu \mathrm{m}$, separation $2000 \mu \mathrm{m}$ ), and mounting the coated coverslips in a standard parallel-plate perfusion chamber ${ }^{38}$, it was possible to determine the thrombus-forming activity of multiple surfaces at the same time (Figure 2A). Tile scans performed after perfusion of $\mathrm{DiOC}_{6}$-labelled whole blood indicated homogeneous adhesion of platelets to the microspots, either as single cells or in aggregates depending on the surface coating (Figure 2B). Control experiments further established that the order of coated proteins in microspots did not affect platelet deposition. For instance, microspotting of fibrinogen, fibronectin or collagen I, at upstream or downstream positions, resulted in the same amount of platelet adhesion and aggregation (Suppl. Figure 1). This pointed to the absence of significant paracrine cross-talk from activated platelets on adjacent microspots.

For a comparative analysis, whole blood from healthy subjects was perfused over microspots (three per run) containing the 52 combinations of substrate proteins and peptides at a high (arterial) wall shear rate of $1600 \mathrm{~s}^{-1}$. Thrombus formation on all surfaces was measured using standardized microscopic procedures (see Methods). Stable platelet adhesion of DiOC $_{6}$-labelled platelets was evaluated from sequential fluorescence images captured in real time during blood flow. Thrombus volume was assessed from z-stacks of confocal images of DiOC 6 fluorescence at end stage. End stage, phase-contrast images were captured to determine overall platelet deposition (surface area coverage) and platelet aggregate size. Activation of the platelets per surface was resolved from confocal fluorescence images after labeling with fluorescein isothiocyanate (FITC)-labelled anti-fibrinogen monoclonal antibody (mAb) (fibrinogen binding, integrin $a_{\mathrm{IIb}} \beta_{3}$ activation), FITC-anti-CD62P mAb (P-selectin expression), or AF647-annexin A5 (procoagulant activity). As 
A

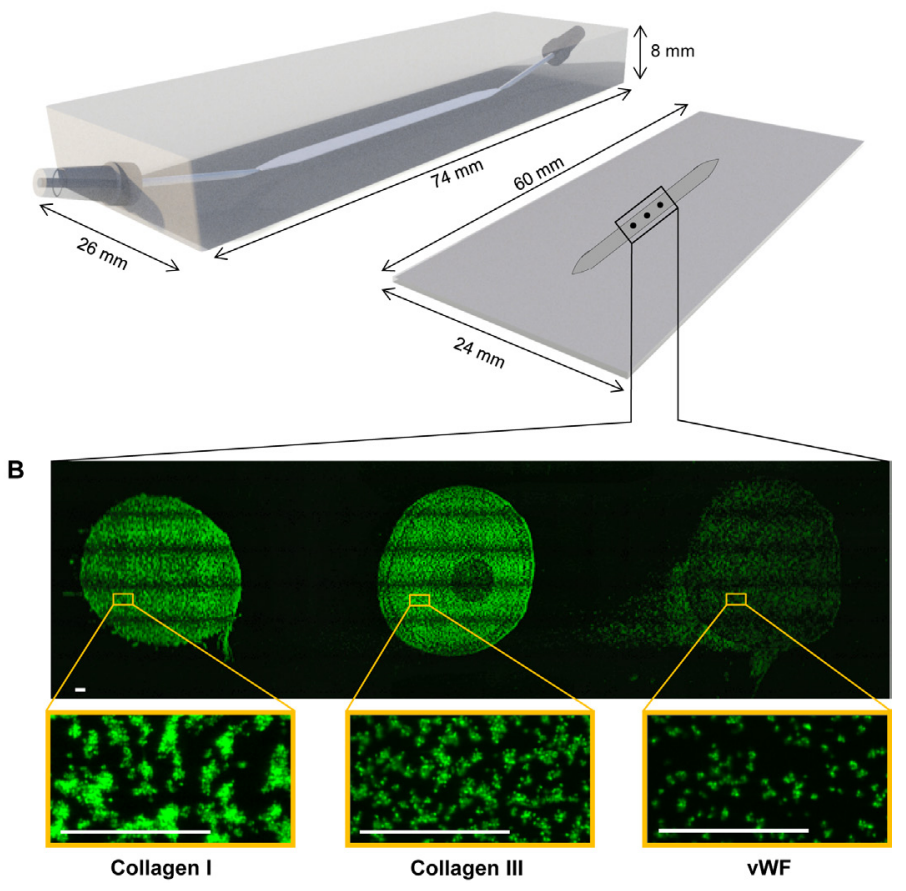

Figure 2. Measurement of thrombus formation on microspot arrays of platelet-adhesive surfaces. (A) Schematic drawing of the used parallel-plate flow chamber (3 mm width, 50 $\mu \mathrm{m}$ depth) and microspot-coated coverslip. Note the small-angular $\left(11^{\circ}\right)$ chamber inlet and outlet, preventing flow perturbations. (B) Distribution of DiOC $_{6}$-labelled platelets adhered to consecutive microspots of collagen type I, collagen type III and vWF, after 3.5 min flow of blood at wall shear rate of $1600 \mathrm{~s}^{-1}$. Given are tile scans of fluorescence images of the full microspots (bar $=100 \mu \mathrm{m}$ ). Black rims are image artifacts due to the tile scanning. Lower panels are enlarged images.

illustrated in Figure 3, platelet adhesion, aggregation and activation markedly differed between the various microspots. No platelets adhered to spots coated with BSA (negative control), while single platelets with low activation state adhered to coated fibrinogen. Small aggregates of platelets with activated integrin $a_{\mathrm{IIb}} \beta_{3}$ and surface expressed P-selectin formed on coated VWF or collagen III. Large platelet aggregates staining for all three activation markers formed on collagen I microspots, as expected ${ }^{39}$.

Control experiments were performed with blocking antibodies (all at $20 \mu \mathrm{g} / \mathrm{mL}$ ) to verify the involvement of specific receptors (Suppl. Table 1) to the flow-dependent deposition of platelets on microspots with single or double coatings (S.M.d.W., personal communication). These antibody experiments affirmed the previously established roles of: GPVI and $a_{2} \beta_{1}$ in thrombus formation on collagen I or III (10B12 Ab against GPVI ${ }^{22,24}$ and mAb 15D7 against integrin $a_{2} \beta_{1}$ (ref. 24)); and GPIb-V-IX for surfaces with VWF or VWF-BP (Fab 6B4 against GPIba ${ }^{24}$ ). The blocking mAb 15D7 had a moderate reducing effect on platelet deposition on surfaces with expected contribution of $a_{2} \beta_{1}$ [VWF/GFOGER-(GPP) ${ }_{n}$,VWF/GFOGER-(GPO) $)_{n}$, i.e., $-42 \%$ and $-28 \%$, respectively $(n=$ $3, P<0.05)$. Blocking experiments with the anti-CD36 antibody Fab-152 gave a reduced platelet response to thrombospondin-1 $(n=3, P<0.05)$, but unchanged platelet deposition to VWF/thrombospondin-1. Other blocking experiments were performed using mAb1976 against integrin $a_{v} \beta_{3}$ and mAb1969 against integrin $a_{5} \beta_{1}(20 \mu \mathrm{g} / \mathrm{mL})$. Single $a_{v} \beta_{3}$-binding (vitronectin, osteopontin) and $a_{5} \beta_{1}$-binding (fibronectin) surfaces did not show platelet adhesion at high shear rate, either in the presence or the absence of the corresponding blocking antibody. In the presence of VWF, the antibodies caused a small but insignificant inhibition of platelet adhesion $(n=3, P>0.06)$. Based on published analyses ${ }^{22,24,37}$, an assignment matrix was constructed of the contribution of individual adhesive receptors to platelet interaction with the different surfaces (Figure 1). Herein, the wellknown VWF-capturing ability of collagens I and III was taken into account.

\section{Identification of thrombus type by multi-parameter analysis}

Replicate measurements of blood perfusion experiments over all 52 coated microspots and using different fluorescent labels ( $n \geq 4$ donors per condition and label) resulted in detailed insight into the contribution of each surface to thrombus formation (see wall chart in Suppl. Figure 2). Standardized analysis of microscopic (fluorescence) images provided the following parameters of 


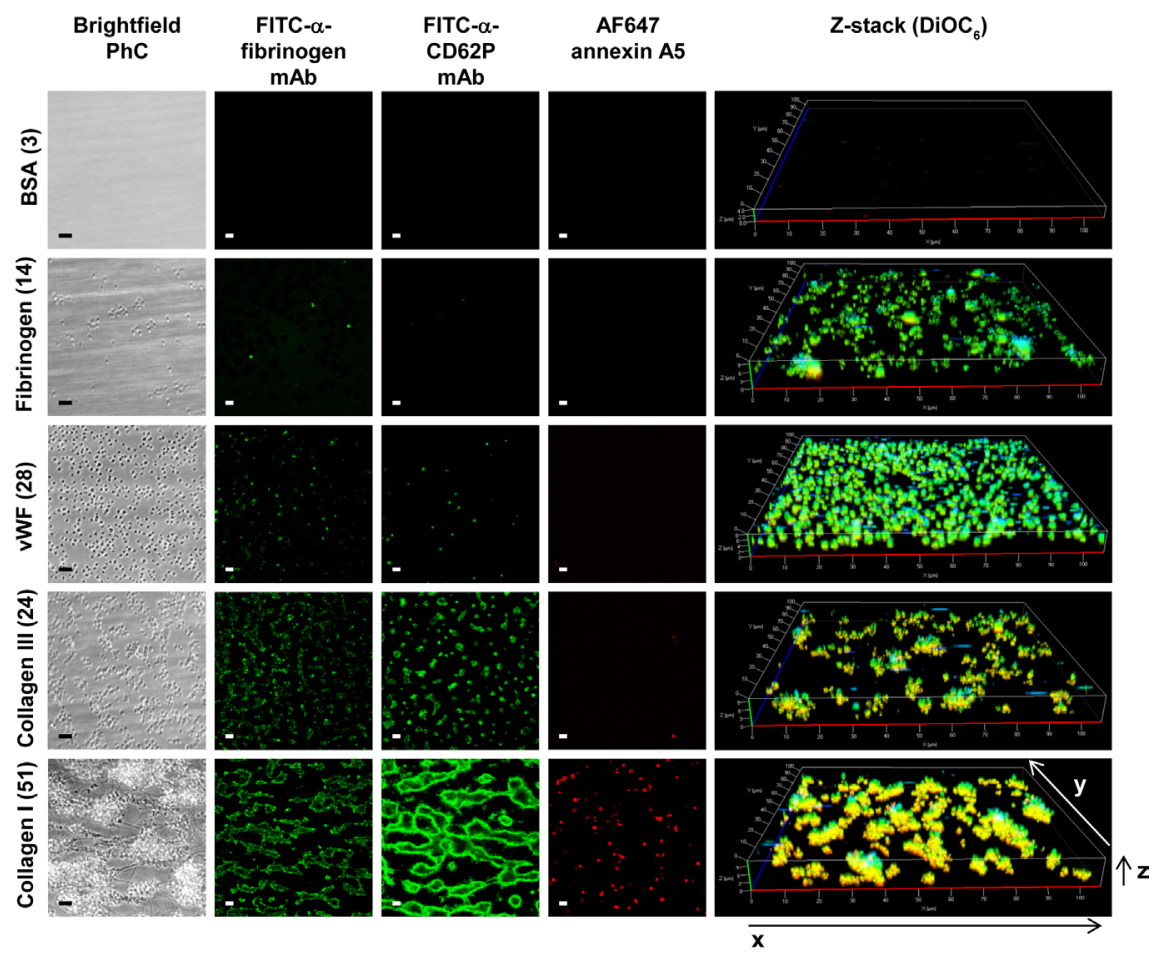

Figure 3. Different types of thrombi formed on various microspots. Blood from control subjects was perfused over microspots with indicated coating for $3.5 \mathrm{~min}$ at $1600 \mathrm{~s}^{-1}$. Shown are representative microscopic images ( $>5$ donors), from left to right: phase-contrast images of adhered platelets (i); fluorescence images of platelets binding FITC-labelled anti-fibrinogen mAb (ii), FITC-labelled anti-CD62P mAb (iii), or AF647-annexin A5 (iv); further, z-stacks from aggregates of DiOC 6 -labelled platelets $(v)$. Bars $=10 \mu \mathrm{m}$. Images from typical surfaces are given (for all 52 surfaces, see Suppl. Figure 1).

thrombus formation: morphological score, integrated feature size, stable platelet adhesion, fibrinogen binding, P-selectin expression, overall platelet deposition, thrombus volume and procoagulant activity. Unsupervised hierarchical cluster analysis of all data (52 microspots, 8 parameters) revealed separation into three patterns of thrombus formation, indicated as types I-III (Figure 4A). Surfaces producing type I thrombi consisted of single-protein coatings causing limited adhesion of few platelets. Type II thrombi mostly formed on surfaces co-coated with VWF or VWF-BP causing deposition of multiple platelets, single or in small aggregates, and showing limited activation (fibrinogen binding, P-selectin expression). Type III thrombi formed on several combined surfaces, giving rise to large aggregates of platelets, high in activation markers. With the exception of collagen I (which binds VWF from plasma), type III thrombi only appeared at double or triple coated surfaces containing VWF, VWF-BP and/or laminin combined with rhodocytin or (GPO) peptides. Robustness of the unsupervised cluster analysis was checked by data re-sampling and rebuilding the tree by 10,000 randomizations with an approximately unbiased $P$ value of 90 , indicative of a strong fit (Figure 4B).

The eight output measurements clustered into adhesion-related parameters (morphological score, integrated feature size, stable platelet adhesion) on the one hand, and activation-related parameters on the other hand. Cohesion of the parameters was confirmed by multiple regression analysis (Table 1), indicating that all eight parameters contributed significantly to the clustering into type I-III thrombi $(P<0.001)$. The strongest coefficients of determination for thrombus type were the linked parameters, morphological score and integrated feature size; the linked parameters, fibrinogen binding, P-selectin expression and platelet deposition; and procoagulant activity. Individually, two parameters, stable platelet adhesion and thrombus volume, were still significantly but less strongly determinative $\left(R^{2}=0.71-0.68\right)$. Complete linkage analysis after removal of the latter two parameters resulted in a cluster plot showing the same division into type I-III thrombi 


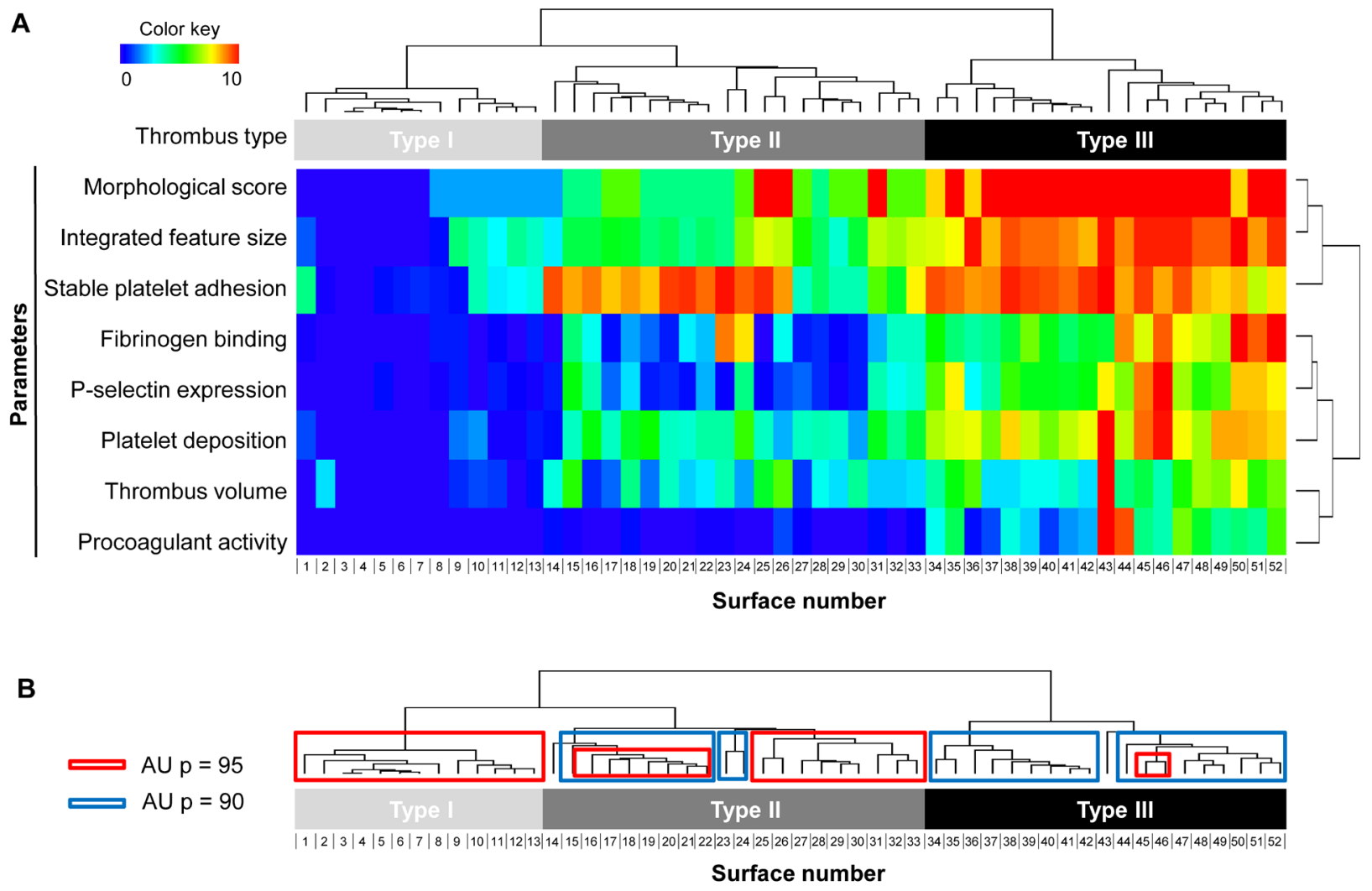

Figure 4. Clustering of thrombus formation at $\mathbf{5 2}$ microspots using $\mathbf{8}$ outcome parameters. Whole blood from control subjects was perfused over arrays of microspots in a parallel-plate flow chamber for 3.5 min at $1600 \mathrm{~s}^{-1}$. Numbering of coatings with different adhesive proteins or peptides as in Figure 1. Recorded phase-contrast images were analyzed for morphological score, integrated feature size and platelet deposition (surface area coverage). Following in situ $\mathrm{DiOC}_{6}$ labeling, fluorescence images were recorded to assess stable platelet adhesion (during blood flow) and thrombus volume (after blood flow). Thrombi were post-stained to determine fibrinogen binding (FITC-labelled anti-fibrinogen mAb), P-selectin expression (FITC-anti-CD62P mAb), and procoagulant activity (AF647-annexin A5). Mean values of the parameters ( $n=5-7$, thrombus size: $n=4-6$ ) were normalized from $0-10$, and arranged by unsupervised hierarchical cluster analysis. (A) Clustered heatmap for 52 different surfaces (columns) and 8 measurement parameters (rows). Clustering of surfaces revealed three different types of thrombus formation, (B) Robustness of data set, assessed by bootstrapping randomizations of all data with Pvclust. Shown are the pro forma clusters obtained, using Approximately Unbiased (AU) $P$ values of 90 and 95, indicative for a strong fit.

(Suppl. Figure 3A-B). Overall, this analysis indicates that the distinction into three types of thrombi is not dependent on the specific measurement parameters. However, a further division into subtypes would be parameter-dependent.

\section{Contributions of VWF and shear rate to thrombus type}

Given the established role of VWF/GPIb in platelet adhesion at high-shear blood flow ${ }^{2,21}$, we performed a sub-analysis of thrombi formed on microspots co-coated with indirectly or directly GPIb-binding substances, that is, VWF-BP or VWF, respectively. The resulting heatmaps of Figure $5 \mathrm{~A}$ show that, for the majority of microspots, the presence of VWF-BP or VWF increased thrombus formation, as assessed from the morphological score, integrated feature size, stable platelet adhesion, platelet deposition and thrombus volume. Values of these parameters significantly increased $(P<0.05$, Student's $t$-test) compared to the surfaces without VWF (-BP), with the exception of microspots containing $a_{6} \beta_{1}$ ligand, laminin (Figure $5 B$ ). Other parameters, such as fibrinogen binding and P-selectin expression, increased to a lesser extent, still significant with co-coated VWF but not VWF-BP. Procoagulant activity was not increased. Overall, the analysis identified most prominent roles of VWF (-BP) on thrombus formation co-coated with, in decreasing order, (assigned receptors 
Table 1. Multiple regression analysis of measurement parameters for the assessment of type I-III thrombi. Given are $R^{2}$ values (Pearson correlation coefficient) with statistical significance for the contribution of each measurement parameter to the type of thrombus formation at shear rate of $1600 \mathrm{~s}^{-1}$ for 52 surfaces ( $150 \mathrm{~s}^{-1}$ for 36 surfaces).

\begin{tabular}{|c|c|c|c|c|}
\hline Parameter & $R^{2}$ & $\boldsymbol{P}$ & $R^{2}$ & $\boldsymbol{P}$ \\
\hline Morphological score & $0.92(0.68)$ & $<0.001$ & & \\
\hline Integrated feature size & $0.91(0.73)$ & $<0.001$ & 0.94 & $<0.001$ \\
\hline Stable platelet adhesion & 0.71 & $<0.001$ & & \\
\hline Fibrinogen binding & $0.73(0.67)$ & $<0.001$ & & \\
\hline P-selectin expression & $0.95(0.68)$ & $<0.001$ & 0.72 & $<0.001$ \\
\hline Platelet deposition & $0.85(0.75)$ & $<0.001$ & & \\
\hline Thrombus volume & 0.68 & $<0.001$ & & \\
\hline Procoagulant activity & $0.79(0.42)$ & $<0.001$ & 0.69 & $<0.001$ \\
\hline
\end{tabular}

in brackets): rhodocytin (CLEC-2) $>(\text { GPO })_{n}\left(\right.$ GPVI), GFOGER-(GPP) $\left(a_{2} \beta_{1}\right)>$ decorin, osteopontin, fibrinogen, fibronectin, vitronectin (integrins $a_{2} \beta_{1}, a_{5} \beta_{1}, a_{I I b} \beta_{3}, a_{v} \beta_{3}$ ) > laminin, thrombospondin-1 $\left(a_{6} \beta_{1}, C D 36\right)$.

To substantiate this further, we also investigated the role of VWF by comparing thrombus formation at high $\left(1600 \mathrm{~s}^{-1}\right)$ and low $\left(150 \mathrm{~s}^{-1}\right)$ wall shear rates, using 36 surfaces. At low shear rate, all six parameters analyzed contributed to formation of type I-III thrombi with high coefficients of determination (Table 1). Heatmaps indicated that, in general, many surfaces that actively supported thrombus formation at wall high shear rate performed less well at low wall shear rate (Figure 6A, $B)$. On the other hand, several of the surfaces less active at high shear rate, particularly those not containing VWF, became more active at lower shear rate. These effects were even more apparent after subtraction analysis (Figure 6C), pointing to improved thrombus formation at high shear rate for all VWF-containing surfaces $(P<0.05)$ on the one hand. Interestingly on the other hand, combinations of laminin $\left(a_{6} \beta_{1}\right)$, rhodocytin (CLEC-2) and GFOGER-(GPO) $\left(\right.$ GPVI, $a_{2} \beta_{1}$ ) provoked high and often increased thrombus formation in the absence of VWF at lower shear rate. Confirmation of these shear-dependent effects was obtained by perfusion studies with 19 surfaces at three wall shear rates of 150,1000 and $1600 \mathrm{~s}^{-1}$. In general, platelet deposition at $1000 \mathrm{~s}^{-1}$ was somewhat lower than at $1600 \mathrm{~s}^{-1}$, the exception being laminin-containing surfaces where highest values were obtained at $1000 \mathrm{~s}^{-1}$ (Suppl. Figure 4).

\section{Receptor combinations in type of thrombus formation}

A partial least-squares regression model was built in Matlab using all output parameters to verify the division of thrombi into three types, determined by unsupervised cluster analysis (Figure 4). In the first model obtained, it was found that two components determined most of the variation $(82.9 \%$ and $7.9 \%)$ in the data set assessing type I, II and III thrombi; furthermore, the six key parameters contributed similarly to this division. This allowed us to calculate mean values of these parameters as a proxy for the type of thrombi formed per surface and wall shear rate (Suppl. Table 2).

A second model was then built, again using partial least-squares regression data analysis, by fitting in the data obtained at low shear rate (which were not used in the first model). This model made it also possible to predict the thrombus type under low shear conditions (Suppl. Table 2). From this analysis it appeared that 6 out of 11 surfaces, which each produced type I thrombi at high shear, formed type II thrombi at low shear. Furthermore, 2 out of eight VWF-containing surfaces producing type III thrombi at high shear formed type II thrombi under low-shear conditions. This class analysis thus confirmed the heatmap subtraction analysis of Figure 6C. 
A

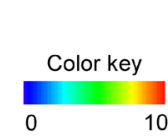

B

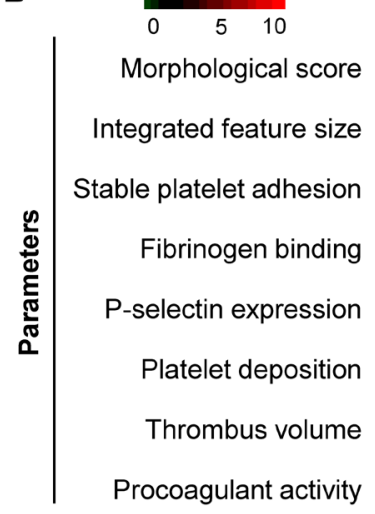

Thrombus type

Color key
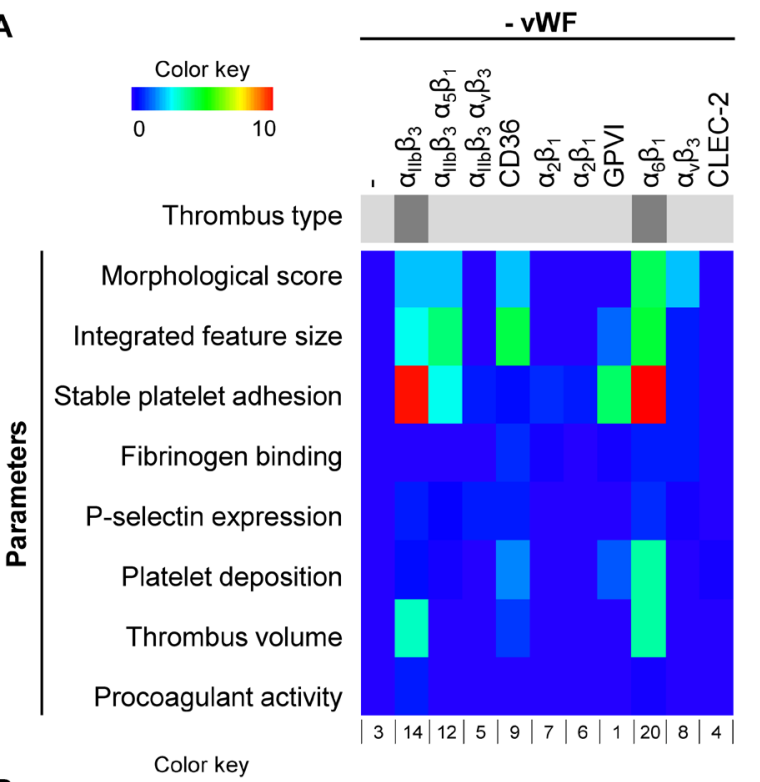

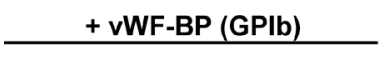

congens

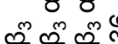

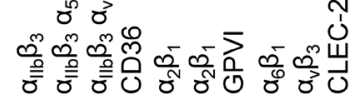
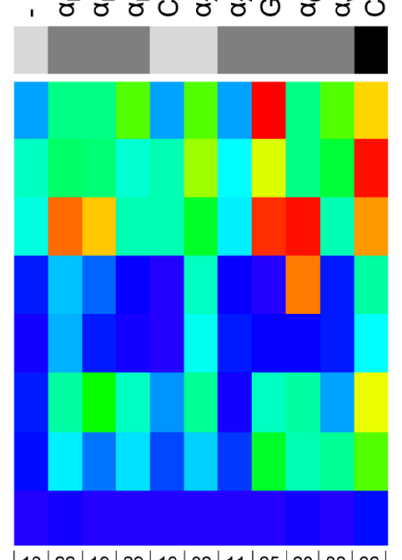

$13|22| 19|29| 10|32| 11|25| 23|30| 36 \mid$ Surface number
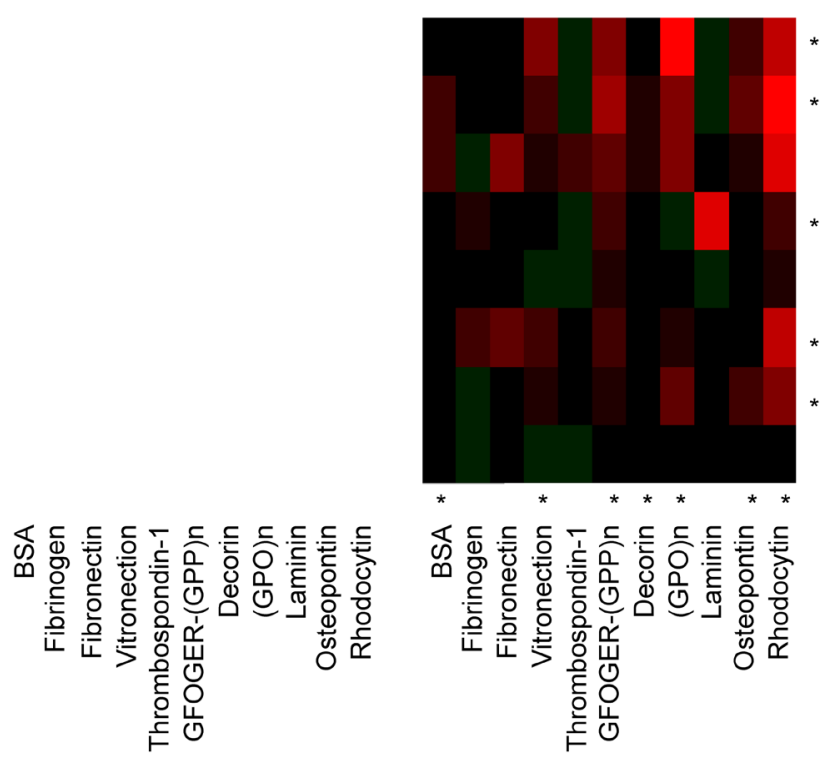

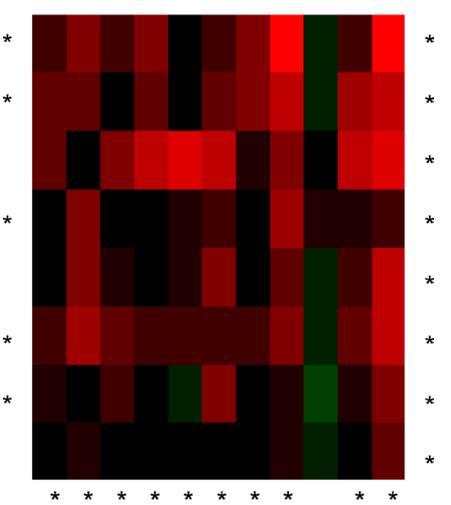

+ vWF (GPIb)

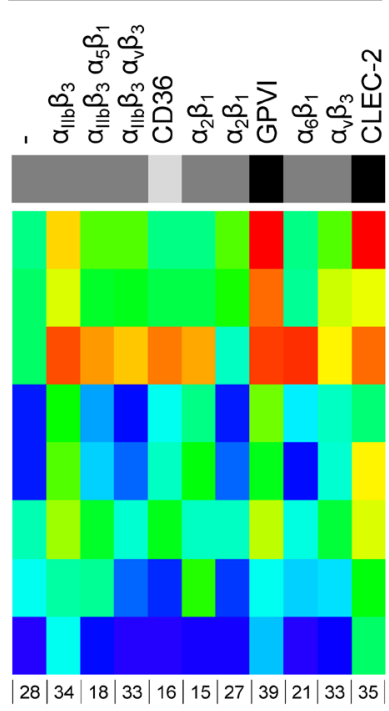

$|28| 34|18| 33|16| 15|27| 39|21| 33 \mid 35$

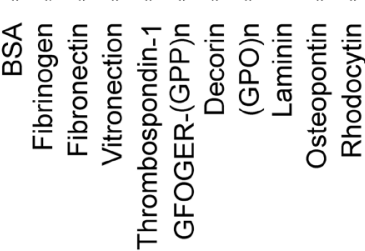

Subtraction

Figure 5. Stimulating effect of co-coating of microspots with VWF-BP or VWF. Whole blood from control subjects was perfused over arrays of microspots for $3.5 \mathrm{~min}$ at $1600 \mathrm{~s}^{-1}$, and analyzed for thrombus formation as in Figure 1. (A) Sub-heat maps of thrombus formation parameters of single coatings (left panel), co-coatings with VWF-BP (middle panel), or co-coatings with VWF (right panel). Formation of type I, II and III thrombi is represented by color bars from gray to black. (B) Subtraction heat maps, indicating the effects of co-coating with VWF-BP (middle) or with VWF (right). Color code is from -1 to $10 . * P<0.05$ (2-tailed Student's t-test) compared to single coating, per row or column.

By combining the analysis of thrombus types and the receptor assignment matrix per surface (Figure 1), we then evaluated the combinations of receptors contributing to formation of type III thrombi. First, computational analysis was performed for the high-shear condition of the surfaces binding GPIb (VWF, VWF-BP) in combination with the key integrins $a_{2} \beta_{1} / a_{6} \beta_{1}$ and the signaling receptors GPVI/CLEC-2. In combination these three receptor/ligand classes consistently produced type III thrombi (Figure 7). Integrins seemed to play a stimulatory but not essential role, with $a_{6} \beta_{1}$ being more active than $a_{2} \beta_{1}$. This was also clear from the mean scores of laminin-containing surfaces $\left(a_{6} \beta_{1}\right.$ binding), which performed better than GFOGER- or decorin-containing surfaces $\left(a_{2} \beta_{1}\right.$ binding) (Suppl. Table 2). 
A
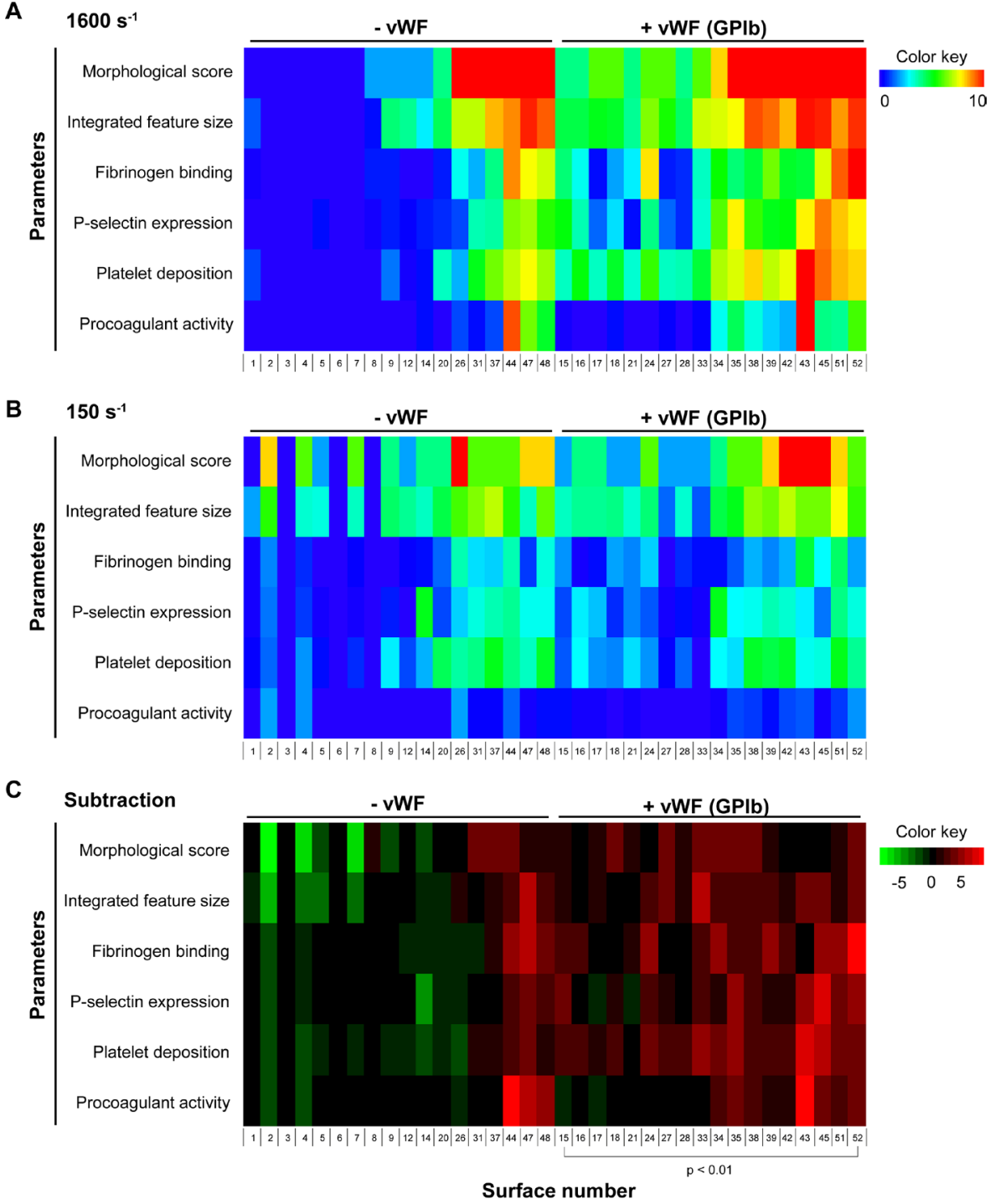

Figure 6. Effect of wall shear rate on thrombus formation on microspot surfaces. Whole blood was perfused over surfaces with or without VWF at indicated wall shear rates. (A) Measured parameters of thrombus formation at 1600 $\mathrm{s}^{-1}\left(2 \times 18\right.$ surfaces, clustering order as in Figure 4). (B) Measurement parameters of thrombus formation at $150 \mathrm{~s}^{-1}$. (C) Linear subtraction heatmapof outcome parameters at low shear rate compared to high shear rate. $* P<0.01$ ( 2 -tailed Student's t-test).
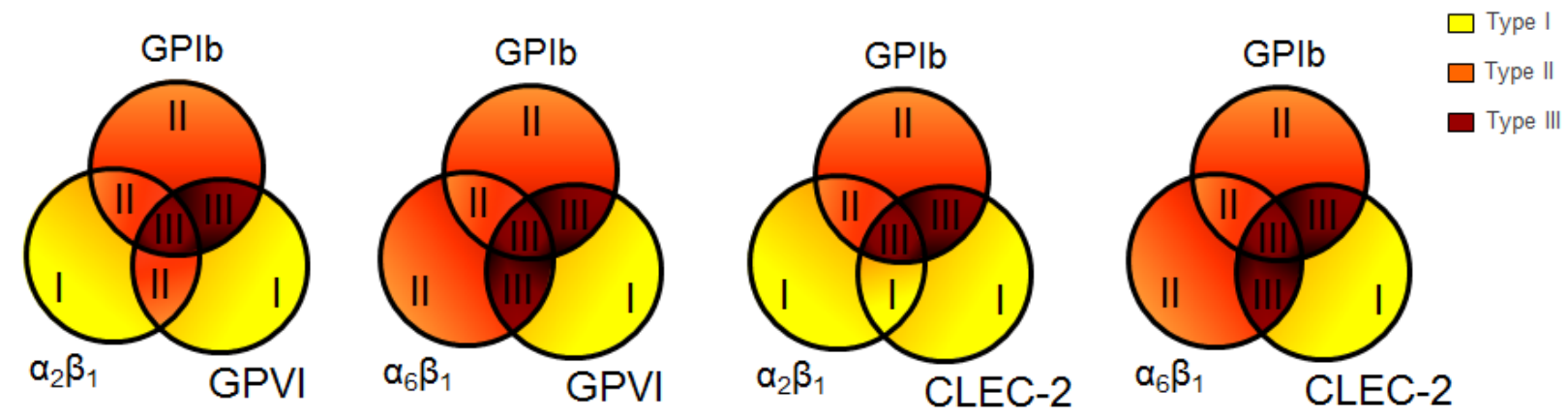

Figure 7. Contribution of platelet-adhesive receptors to formation of type I-III thrombi. Schematic representation of thrombus type formed at high shear rate on surfaces capturing platelets via adhesive receptors (GPIb, and integrins $a_{2} \beta_{1}, a_{6} \beta_{1}$ ) as well as signaling-linked receptors (GPVI, CLEC-2). 


\begin{tabular}{lcc}
\hline Receptor & \multicolumn{2}{c}{ Weight factor } \\
& $1600 \mathrm{~s}^{-1}$ & $150 \mathrm{~s}^{-1}$ \\
\hline$\beta$ constant & 0.947 & \\
GPIb-V-IX & 0.687 & 0.049 \\
GPVI & 0.858 & 0.903 \\
CLEC-2 & 0.763 & 0.964 \\
$\mathrm{a}_{6} \beta_{1}$ & 0.653 & 0.879 \\
$\mathrm{a}_{\mathrm{IIJ}} \beta_{3}$ & 0.527 & 0.877 \\
$\mathrm{a}_{2} \beta_{1}$ & 0.193 & 0.165 \\
CD36 & -0.262 & 0.898 \\
$\mathrm{a}_{5} \beta_{1}$ & -0.102 & -0.834 \\
$\mathrm{a}_{\mathrm{v}} \beta_{3}$ & -0.159 & -0.746 \\
\hline
\end{tabular}

Table 2. Predicted contribution of platelet receptors in formation of type III thrombi at high shear rate. Beta matrix values after principal component analysis of weight factors, predicting the contribution of individual adhesive receptors to formation of type III thrombi. The model built for shear rate of $1600 \mathrm{~s}^{-1}$ was based on 52 surfaces and 6 parameters ( $71 \%$ of variance). A separate scaled model was built for shear rate of $150 \mathrm{~s}^{-1}$, based on 36 surfaces and 6 parameters (58\% of variance). Both models used the assignment matrix of Figure 1. Note that negative values designate relative inactivity of the receptors to participate in type III thrombus formation.

A similar analysis was performed by partial least-squares regression analysis linking the various receptors to thrombus type (high shear rate). For the 52 surfaces of the main heatmap, a new model was built with 3 components (together $72 \%$ of the variance), which after rounding gave three predicted thrombus types. A confusion matrix indicated that only 10 of the 52 samples appeared in the wrong class, with miss-assignment predominantly ( 8 out of 10 ) in the lower classes of thrombi (type I-II), where severity was overestimated. This model was confirmed by cross validation. Using the receptor assignments per surface, a $\beta$-weight matrix was constructed to calculate the contribution of each receptor to the type of thrombus formation (Table 2). The matrix confirmed prominent differences in contribution of the various receptors, pointing to major roles for GPIb, the signaling receptors GPVI and CLEC-2, as well as for the integrins $a_{6} \beta_{1}$ and $a_{\text {IIb }} \beta_{3}$. While contribution of $a_{2} \beta_{1}$ is less pronounced, the other integrins $a_{5} \beta_{1}$ and $a_{v} \beta_{3}$ along with CD36 score lowest. Note that negative values in the matrix only indicate relative inability of the receptor to contribute to type III thrombus formation. Overall, these findings identify highly stimulatory roles of CLEC- 2 and $a_{6} \beta_{1}$ in type III thrombus formation at high shear rate, in addition to the established roles of GPIb, GPVI and $a_{2} \beta_{1}$.

Using the same receptor assignments and partial least-squares regression analysis, a new $\beta$ - matrix was built to predict the contributions of individual receptors at low shear rate, that is, by clustering the low-shear data into the matrix of high-shear data. Cross-validation predictions showed that only 7 of the 36 surfaces were wrongly predicted for thrombus type. The resulting $\beta$-weight factors ( 2 components, $58 \%$ of the variance) pointed to a minimized contribution of GPIb to thrombus type at low shear rate (Table 2). Further, this analysis indicated similarly high contributions of GPVI, CLEC-2, $a_{6} \beta_{1}$ and $a_{\mathrm{IL}} \beta_{3}$. At low shear rate, CD36 had a positive role, while $a_{v} \beta_{3}$ and $a_{5} \beta_{1}$ again did not contribute to formation of type III thrombi. Finally, the model was rebuilt by exclusion of a contribution of GPIb at low shear rate, giving essentially the same results (only 4 out of 36 surfaces wrongly predicted). This calculated absence of the GPIb-V-IX at low-shear flow conditions is in good agreement with the literature ${ }^{2}$.

\section{Platelet function disorders leading to impaired thrombus formation}

A panel of nine microspot surfaces (all major receptors and VWF co-coated) was selected to determine reference values of all parameters for $n \geq 6$ healthy subjects (Suppl. Table 3 ). To assess intra-individual variability, blood samples were analyzed from six healthy volunteers, taken each at four different days. For all surfaces together: intra-individual coefficient of variations (CVs) 


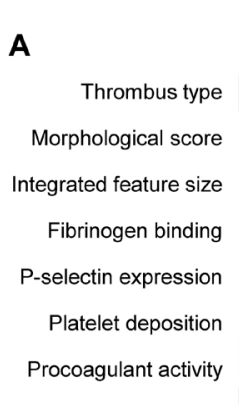

B Morphological score
Integrated feature size
Fibrinogen binding
P-selectin expression
Platelet deposition
Procoagulant activity

C Morphological score
Integrated feature size
Fibrinogen binding
P-selectin expression
Platelet deposition
Procoagulant activity (1)
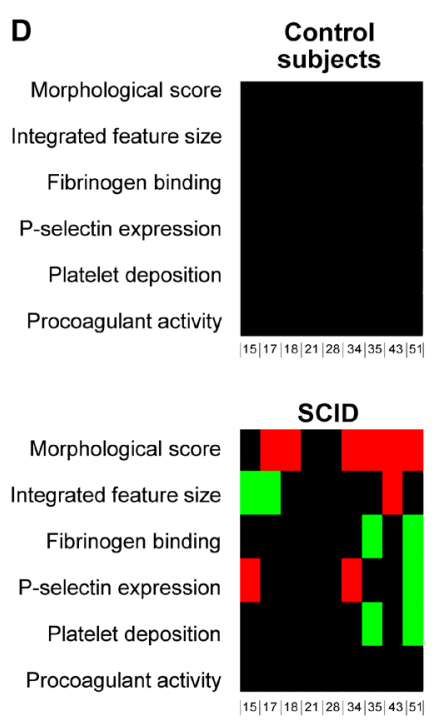
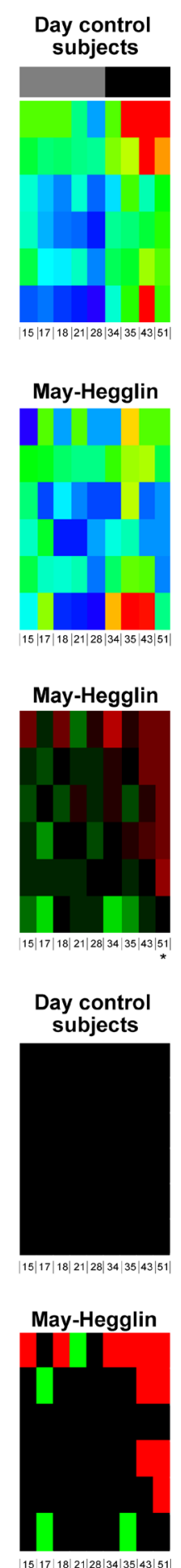
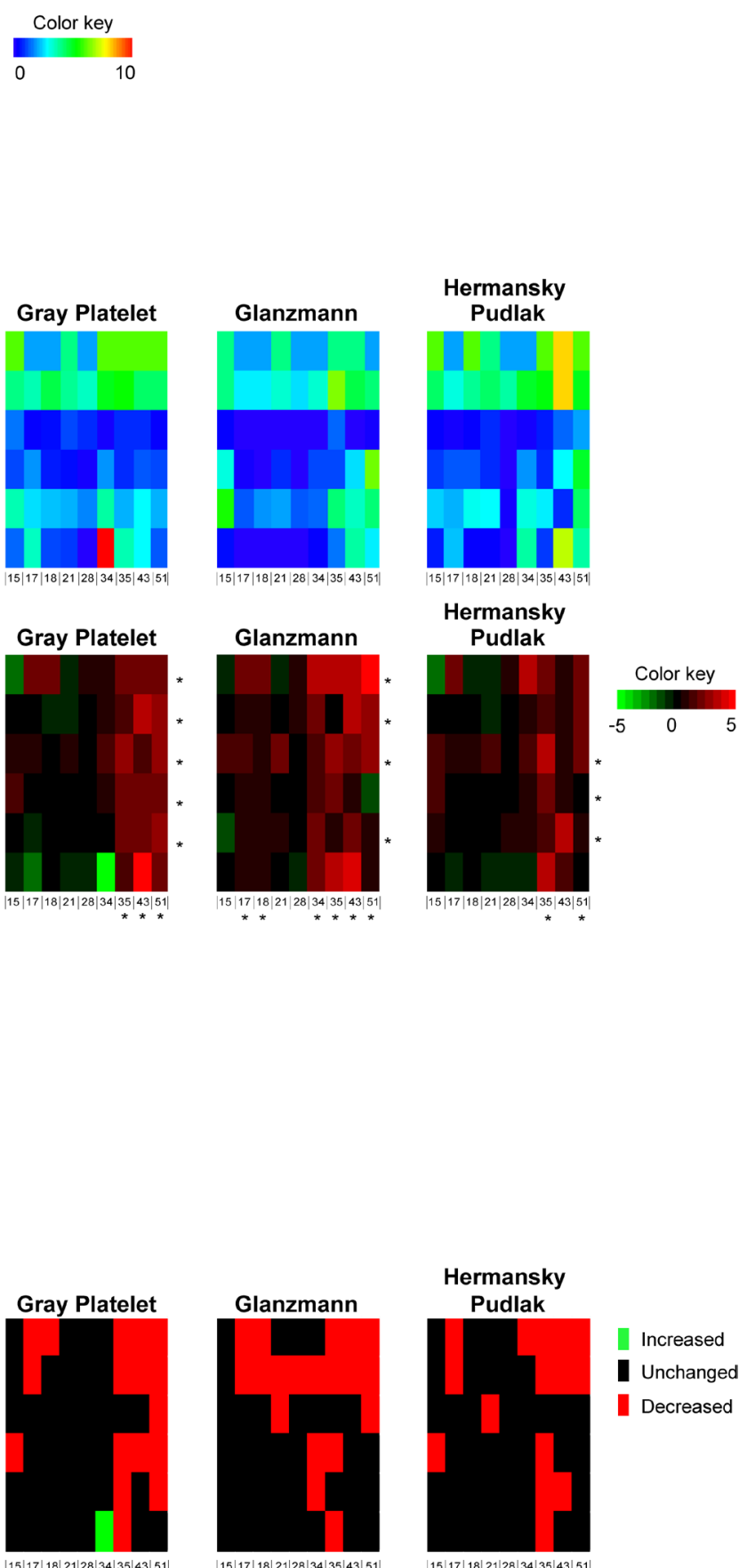

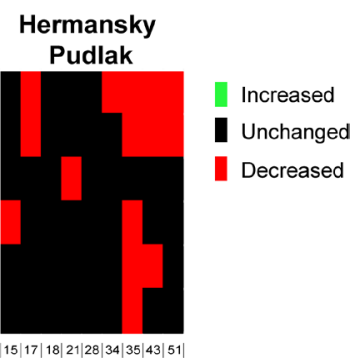

Figure 8. Abnormal thrombus formation in blood from patients with rare platelet function defects. Whole blood from controls subjects or indicated patients was perfused over microspots of nine different surfaces (three microspots per run, 2-3 runs per surface). Surface numbering as in Figure 1. (A) Heatmap of average parameters of thrombus formation for blood samples from control subjects (left), and blood samples from day control subjects (right). (B) Heatmaps and (C) subtraction heatmaps of parameters of thrombus formation for patients with indicated syndromes: SCID, May-Hegglin anomaly, grey platelet syndrome, Glanzmann's thrombasthenia or Hermansky-Pudlak syndrome. $* \mathrm{P}<0.05$ compared with control subjects (two-tailed Student's t-test). (D) Significance map of parameters from a representative control subject and a day control subject, as well as from all patients. Colour key: red=deviating $<$ mean -2 $\mathrm{SD}$; green=deviating $>$ mean $+2 \mathrm{SD}$, relative to normal values. Thrombus formation was also assessed in blood from rare patients with established platelet function disorders. Heatmap mean data were generated for the control subjects and the individual patients under investigation (Figure $8 \mathrm{~A}, \mathrm{~B}$ ). These data were further evaluated in subtraction heat maps and significance maps (Figure 8C, D). 
for the morphological score (5.7\%) were 2.0 times lower than inter-individual CVs (11.2\%); for platelet deposition, intra-individual CVs (10.8\%) were 3.1 times lower than inter-individual CVs (33.7\%). Similarly, for the platelet activation markers, that is, fibrinogen binding, P-selectin expression and phosphatidylserine (PS) exposure, intra-individual CVs were 3.4 times, 2.6 times and 3.5 times lower, respectively, than the corresponding inter-individual CVs.

Thrombus formation was also assessed in blood from rare patients with established platelet function disorders. Heatmap mean data were generated for the control subjects and the individual patients under investigation (Figure $8 \mathrm{~A}, \mathrm{~B}$ ). These data were further evaluated in subtraction heat maps and significance maps (Figure $8 \mathrm{C}, \mathrm{D}$ ).

Thrombus formation was assessed in blood from a patient with severe immune deficiency syndrome (SCID) ${ }^{40}$, associated with near-complete deficiency in store-induced calcium entry in hematopoietic cells including platelets. In spite of a reduced morphological score of thrombus formation, platelet activation parameters on surfaces producing type III thrombi, that is, VWF/ rhodocytin and collagen I, were increased in comparison to control subjects (Figure 8D, E). This may point to increased CLEC-2- and GPVI-dependent platelet activation under high shear flow, for example compensating for absence of one of the calcium entry pathways. Blood samples were also examined from a patient with May-Hegglin anomaly ${ }^{41}$, characterized by a myosin cytoskeletal defect and macrothrombocytopenia, in which disease the consequences for platelet function are not well understood. Thrombus formation parameters were mostly within the normal range, with the exception of reduced platelet aggregate formation (morphological score, integrated feature size, platelet deposition) on GPVI-binding surfaces (VWF, VWF/GFOGER-(GPO) ${ }_{n}$, collagen I). Most values for non-GPVI-binding surfaces were in the normal range. However, platelet procoagulant activity tended to be higher on two-component surfaces.

Also investigated was blood from a patient with gray platelet syndrome ${ }^{41}$, phenotyped with a partial deficiency in platelet a-granules. The thrombi formed were reduced in most parameters, with the exception of procoagulant activity, typically on type III-inducing surfaces, that is, VWF/ rhodocytin, VWF/GFOGER-(GPO) and collagen I (Figure 8D). P-selectin expression was markedly reduced on all surfaces, as expected. Overall, these results may point to impaired thrombus formation due to reduced a-granule release, in particular on surfaces activating via CLEC-2 or GPVI. Furthermore, testing the blood from a patient with Glanzmann's thrombasthenia (deficiency in $\mathrm{a}_{\mathrm{IIb}} \beta_{3}$ ) resulted in a significant reduction in platelet aggregation tendency (morphological score, integrated feature size and/or fibrinogen binding) on most surfaces, thus substantiating $\mathrm{a}_{\mathrm{IIb}} \beta_{3}$ as being invariably implicated in platelet-platelet interactions. Platelet activation parameters (P-selectin expression, platelet deposition) were most prominently reduced on VWF/fibrinogen surface. A final blood sample was used from a patient with Hermansky-Pudlak syndrome ${ }^{41}$, characterized by absence of dense granules. In this case, parameters of thrombus formation decreased with VWF/ vitronectin, VWF/rhodocytin, VWF/GFOGER-(GPO) $)_{n}$ and collagen I. Thrombus formation hence diminished on surfaces binding GPIb plus $a_{v} \beta_{3}$ CLEC-2 or GPVI. For all patients together, thrombus formation was mostly altered on VWF-containing surfaces with rhodocytin, GFOGER-(GPO) collagen I; the most affected parameters were morphological score, integrated feature size and fibrinogen binding.

\section{Additional role of thrombin}

To determine the role of thrombin, as a potent platelet agonist in thrombus formation, recalcified blood samples were flowed over microspots containing surfaces co-coated with tissue factor. Corn trypsin inhibitor and GPRP were added to prevent contact activation and fibrin polymerization, respectively. Representative results are given in Suppl. Table 4. On a VWF/fibronectin surface, all parameters of thrombus formation increased in the presence of thrombin generation. However, on coated collagen I, platelet deposition and fluorescence markers of platelet activation parameters appeared to decrease in the presence of thrombin. This paradoxical effect was due to contraction 
of the thrombi on collagen, resulting in lower surface area coverage of the (labelled) platelets in the presence of thrombin. Incidental presence of fibrin clots on the surfaces made this assay variant less suitable for standardization. Overall, these data pointed to a more complex way of thrombus phenotyping in the presence of thrombin, which requires further evaluation in the future.

\section{Discussion}

The present results aim to contribute to the recognized high need for full standardization and exploitation of flow assays for integrative whole blood platelet function testing ${ }^{9}$. By systematic assessment of thrombus formation under high shear flow on 52 microspot surfaces using eight outcome parameters, we have identified the most determinative parameters of this process. These reflect overall platelet adhesion and aggregation (morphological score, integrated feature size, platelet deposition), and determinants of platelet activation (fibrinogen binding, P-selectin expression, procoagulant activity). Leaving out two parameters - stable platelet adhesion and thrombus volume - did not change the initial clustering of surfaces with different types of thrombi. The remaining six parameters contributed equally to the thrombus-forming process, and were hence combined in least-squares regression analyses to build a model for the formation of thrombi with different phenotypes. The division into three thrombus types was almost identical to that achieved by unsupervised cluster analysis of all data ( 52 surfaces, 8 parameters).

The cluster analysis indicated a robust distinction of surfaces into three classes, supporting: adhesion of few single platelets (type I); extensive adhesion with small aggregates and minimal platelet activation (type II); or large aggregates with fully activated platelets (type III). Thrombi of type III were only present on combined microspots containing VWF or VWF-BP, an exception being collagen I which binds VWF from plasma; other necessary components were laminin (binding $a_{6} \beta_{1}$ ), peptides containing (GPO) (binding GPVI) or rhodocytin (binding CLEC-2). Other adhesive proteins tested appeared to be less active in combined surfaces (fibrinogen > osteopontin, collagen III > fibronectin, vitronectin, thrombospondin-1, decorin). Note that the CLEC-2 ligand podoplanin is not present in the arterial wall or in plasma ${ }^{35}$.

A particular role in high-shear thrombus formation is played by VWF, because it binds to two abundant platelet receptors, GPIb and $\mathrm{a}_{\mathrm{IIb}} \beta_{3}$ and to several matrix proteins ${ }^{2}$, that is, fibrillar collagens (via its $A 3$ domain) ${ }^{19}$ and, as recently demonstrated, to laminins ${ }^{42}$. The latter finding explains why we could not identify cooperative roles of VWF and laminin on microspots and high activity of laminin/rhodocytin co-coatings. For the majority of other surfaces, co-coating of VWF (or VWF-BP) increased the parameters of thrombus formation at high shear rate, but not at low shear rate. The platelet-activating effect of surface-immobilized laminin has been observed before under stasis, and was attributed to $a_{6} \beta_{1}$ and GPVI ${ }^{32,43}$. However, using GPVI inhibitors we did not find a role of GPVI in flow-dependent platelet adhesion and activation on laminin. Our data with microspotted peptides agree with the earlier conclusion that synthetic peptides designed to mimic platelet-collagen interactions via GPVI, VWF/GPIb and $a_{2} \beta_{1}$, namely (GPO), VWF-BP and GFOGER peptides, can completely replace native collagen fibers in supporting thrombus formation ${ }^{24,37,44}$.

Regression models built to predict the involvement of different platelet receptors to full thrombus formation yielded interesting results. In addition to the established contribution of GPIb and GPVI, we find major roles for CLEC-2 and the integrins $a_{6} \beta_{1}$ and $a_{\mathrm{IIb}} \beta_{3}$. At high shear rate, platelet adhesion via integrin $a_{2} \beta_{1}$ contributed less to type III thrombus formation, while other receptors $a_{v} \beta_{3}, a_{5} \beta_{1}$ or CD36 were even less effective. This conclusion is in full accordance with the major and additive roles of GPVI, CLEC-2 and integrin $a_{6} \beta_{1}$ in arterial thrombus formation in established mouse models of arterial thrombus formation in vivo ${ }^{35,42}$. Importantly, fitting the low shear data into these regression models pointed to an almost annulled contribution of GPIb, which is in accordance with the current concept of the GPIb-V-IX complex as key receptor for platelet adhesion at high-shear conditions ${ }^{2}$. Although these results indicate a strong role of this complex in the formation of type III thrombi at high shear, they do not prove that GPIb itself triggers signal- 
ing events in platelets. According to the built model, the other most remarkable change in weight factors at low shear rate is a positive contribution of CD36.

Normal values were determined for nine surfaces and six outcome parameters. In particular, the parameters fibrinogen binding, P-selectin expression, platelet deposition and platelet procoagulant activity showed high inter-sample variation. High inter-individual variability in platelet deposition on collagen surfaces has also been reported by others, and could be correlated to plasma levels of VWF, platelet count, hematocrit, sex and platelet receptor genotype ${ }^{14}$. Using different types of microfluidic devices, it was also possible to correlate outcome parameters of thrombus formation to platelet calcium responses (under stasis) ${ }^{15}$, and to aspirin and/or clopidogrel intake in patients with heart disease ${ }^{16}$. Taken together, the inter-individual variability in this kind of whole blood measurements seems to be linked, at least in part, to clinically relevant determinants of platelet function in cardiovascular disease.

Consistent results were obtained upon application of the multi-parameter microspot assay to blood from patients with established platelet deficiencies in platelet functions. With the exception of blood from the SCID patient, the overall effect of disease on all parameters was reduced thrombus formation (9-18 out of 54 parameters reduced) (Figure 8D). Spots of collagen I, VWF/ GFOGER-(GPO) $)_{n}$ and VWF/rhodocytin were most discriminative, all normally resulting in type III thrombi. Of the type II thrombus-inducing surfaces, only VWF/vitronectin showed two reduced parameters for the majority of patients. Together, the patient data indicate that this multi-parameter, multi-surface test detects the consequences of actin cytoskeleton alterations (May-Hegglin), deficient alpha or dense granule secretion (gray platelet, Hermansky-Pudlak), and impaired $\mathrm{a}_{\mathrm{II}} \beta_{3}$ activity (Glanzmann). However, in the case of impaired store-operated calcium entry (SCID patient), we find a similar number of parameters decreased and increased.

In conclusion, we developed a standardized procedure to systematically test thrombus formation upon whole blood perfusion over arrays of microspotted adhesive surface. Using systems biology approaches, we built a model predicting the roles of platelet receptors in shear-dependent thrombus formation, and generated templates to determine aberrations in this process in patients with platelet dysfunctions. This knowledge of surfaces and output parameters is pivotal in the planned design of microscope-independent flow devices. Applications of this advanced technique are numerous, not only in the profiling of patients with a (suspected) bleeding disorder or a pro-thrombotic tendency, but also in the monitoring of functional aberrations in platelet count and of antiplatelet therapy. Furthermore, in a modified way, it can be used to assess the platelet-adhesive properties under flow of blood-derived leukocytes, natural stem cells and malignant cells in cancer.

\section{Acknowledgments}

This work was supported by grants from the Center for Translational Molecular Medicine (INCOAG), the Dutch Heart Foundation (2011T6), the Landsteiner Foundation for Blood Transfusion Research (1006) and ZonMW (MKMD 114021004). We thank R. Verdoold and L. Baaten for help with figure preparations.

\section{References}

1. Jackson SP. Arterial thrombosis: insidious, unpredictable and deadly. Nat Med 17, 1423-1436 (2011).

2. Rugger, ZM, Mendolicchio GL. Adhesion mechanisms in platelet function. Circ Res 100, 1673-1685 (2007).

3. Jackson SP. The growing complexity of platelet aggregation. Blood 109, 5087-5095 (2007).

4. Versteeg HH, Heemskerk, JW, Levi M, Reitsma PS. New fundamentals in hemostasis. Physiol Rev 93, 327-358 (2013).

5. Sakariassen KS, Muggli R, Baumgartner HR. Measurements of platelet interaction with components of the vessel wall in flowing blood. Meth Enzymol 169, 37-70 (1989).

6. Savage B \& Ruggeri ZM. Selective recognition of adhesive sites in surface-bound fibrinogen by glyco- 
protein IIb-IIIa on nonactivated platelets. J Biol Chem 266, 11227-11233 (1991).

7. Konopatskaya O, Matthews SA, Harper MT, Gilio K, Cosemans JM, Williams CM, Navarro MN, Carter DA, Heemskerk JW, Leitges M, Cantrell D, Poole AW. Protein kinase C mediates platelet secretion and thrombus formation through protein kinase D2. Blood 118, 416-424 (2011).

8. Stegner D, Nieswandt B. Platelet receptor signaling in thrombus formation. J Mol Med 89, 109-121 (2011).

9. Roest M, Reininger A, Zwaginga JJ, King MR, Heemskerk JW. Flow chamber-based assays to measure thrombus formation in vitro: requirements for standardization. J Thromb Haemost 9, 2322-2324 (2011).

10. Okorie UM, Diamond SL. Matrix protein microarrays for spatially and compositionally controlled microspot thrombosis under laminar flow. Biophys J 91, 3474-3481 (2006).

11. Grabowski EF, Yam K, Gerace M. Evaluation of hemostasis in flowing blood. Am J Hematol 87, S51-55 (2012).

12. Tsai M, Kita A, Leach J, Rounsevell R, Huang JN, Moake J, Ware RE, Fletcher DA, Lam WA. In vitro modeling of the microvascular occlusion and thrombosis that occur in hematologic diseases using microfluidic technology. J Clin Invest 122, 408-418 (2011).

13. Westein E, de Witt S, Lamers M, Cosemans JM, Heemskerk JW. Monitoring in vitro thrombus formation with novel microfluidic devices. Platelets 23, 501-509 (2012).

14. Neeves KB, Onasoga AA, Hansen RR, Lily JJ, Venckunaite D, Sumber MB, Irish AT, Brodsky G, Manco-Johnson MJ, Di Paola JA. Sources of variability in platelet accumulation on type 1 fibrillar collagen in microfluidic flow assays. Plos One 7, e54680 (2013).

15. Flamm MH, Colace TV, Chatterjee MS, Jing H, Zhou S, Jaeger D, Brass LF, Sinno T, Diamond SL. Multiscale prediction of patient-specific platelet function under flow. Blood 120, 190-198 (2012).

16. Hosokawa K, Ohnishi T, Sameshima H, Miura N, Ito T, Koide T, Maruyama I. Analysing responses to aspirin and clopidogrel by measuring platelet thrombus formation under arterial flow conditions. Thromb Haemost 109, 102-111 (2013).

17. Mendolicchio GL, Zavalloni D, Bacci M, Corrada E, Marconi M, Lodigiani C, Presbitero P, Rota L, Ruggeri ZM. Variable effect of $\mathrm{P}_{2} \mathrm{Y}_{12}$ inhibition on platelet thrombus volume in flowing blood. J Thromb Haemost 9, 373-382 (2011).

18. Harrison P, Mackie I, Mumford A, Briggs C, Liesner R, Winter M, Machin S. Guidelines for the laboratory investigations of heritable disorders of platelet function. Br J Haematol 155, 30-44 (2011).

19. Farndale RW, Sixma JJ, Barnes MJ, de Groot PG. The role of collagen in thrombosis and hemostasis. J Thromb Haemost 2, 561-573 (2004).

20. Gibbins JM. Platelet adhesion signalling and the regulation of thrombus formation. J Cell Sci 117, 3415-3425 (2004).

21. Savage B, Saldivar E, Ruggeri ZM. Initiation of platelet adhesion by arrest onto fibrinogen or translocation on von Willebrand factor. Cell 84, 289-297 (1996).

22. Siljander P, Munnix IC, Smethurst PA, Deckmyn H, Lindhout T, Ouwehand WH, Farndale RW, Heemskerk JW. Platelet receptor interplay regulates collagen-induced thrombus formation in flowing human blood. Blood 103, 1333-1341 (2004).

23. Auger, J. M., Kuijpers, M. J., Senis, Y. A., Watson, S. P., Heemskerk, J. W. Adhesion of human and mouse platelets to collagen under shear: a unifying model. Faseb J 19, 825-827 (2005).

24. Munnix IC, Gilio K, Siljander PR, Raynal N, Feijge MA, Hackeng TM, Deckmyn H, Smethurst PA, Farndale RW, Heemskerk JW. Collagen-mimetic peptides mediate flow-dependent thrombus formation by high- or low-affinity binding of integrin $a_{2} \beta_{1}$ and glycoprotein VI. J Thromb Haemost 6, 2132-2142 (2008).

25. Van de Walle GR, Schoolmeester A, Iserbyt BF, Cosemans JM Heemskerk JW, Hoylaerts MF, Nurden A, Vanhoorelbeke $K$, Deckmyn $H$. Activation of $a_{\mathrm{IIb}} \beta_{3}$ is a sufficient but also an imperative prerequisite for activation of $a_{2} \beta_{1}$ on platelets. Blood 109, 595-602 (2007).

26. Heemskerk JW, Mattheij N, Cosemans JM. Platelet-based coagulation: different populations, different functions. J Thromb Haemost 11, 2-11 (2013).

27. Endenburg SC, Lindeboom-Blokzijl L, Zwaginga JJ, Sixma JJ, de Groot PG. Plasma fibrinogen inhibits platelet adhesion in flowing blood to immobilized fibrinogen. Arterioscler Thromb Vasc Biol 16, 633638 (1996).

28. Chada $\mathrm{D}$, Mather T, Nollert MU. The synergy site of fibronectin is required for strong interaction with the platelet integrin $\mathrm{a}_{\mathrm{IIb}} \beta_{3}$. Ann Biomed Engin 34, 1542-1552 (2006).

29. Asch E, Podack E. Vitronectin binds to activated human platelets and plays a role in platelet aggregation. J Clin Invest 85, 1372-1378 (1990). 
30. Wu YP, Bloemendal HJ, Voest EE, Logtenberg T, de Groot PG, Gebbink MF, de Boer HC. Fibrin-incorporated vitronectin is involved in platelet adhesion and thrombus formation through homotypic interactions with platelet-associated vitronectin. Blood 104, 1034-1041 (2004).

31. Inoue O, Suzuki-Inoue K, McCarty OJ, Moroi M, Ruggeri ZM, Kunicki TJ, Ozaki Y, Watson SP. Laminin stimulates spreading of platelets through integrin $a_{6} \beta_{1}$-dependent activation of GPVI. Blood 107, 1405-1412 (2006).

32. Inoue O, Suzuki-Inoue K, Ozaki Y. Redundant mechanism of platelet adhesion to laminin and collagen under flow: involvement of von Willebrand factor and glycoprotein Ib-IX-V. J Biol Chem 283, 1627916282 (2008).

33. Jurk K, Clemetsom KJ, de Groot PG, Brodde MF, Steiner M, Savion N, Varon D, Sixma JJ, van Aken H, Kehrel BE. Thrombospondin-1 mediates platelet adhesion at high shear via glycoprotein Ib (GPIb): an alternative/backup mechanism to von Willebrand factor. Faseb J 17, 1490-1492 (2003).

34. May F, Hagedorn I, Pleines I, Bender M, Vogtle T, Eble J, Elvers M, Nieswandt B. CLEC-2 is an essential platelet-activating receptor in hemostasis and thrombosis. Blood 114, 3464-3472 (2009).

35. Suzuki-Inoue K, Inoue O, Ding G, Nishimura S, Hokamura K, Eto K, Kashiwagi H, Tomiyama Y, Yatomi Y, Umemura K, Shin Y, Hisashima M, Ozaki Y. Essential in vivo roles of the C-type lectin receptor CLEC2: embryonic/neonatal lethality of CLEC-2-deficient mice by blood/lymphatic misconnections and impaired thrombus formation of CLEC-2-deficient platelets. J Biol Chem 285, 24494-24507 (2010).

36. Severin S, Pollit AY, Navarro-Nunez L, Nash CA, Mourao-Sa D, Elbe JA, Senis YA, Watson SP. Syk-dependent phosphorylation of CLEC-2: a novel mechanism of hem-immunoreceptor tyrosine-based activation motif signaling. J Biol Chem 286, 4107-4116 (2011).

37. Pugh N, Simpson AM, Smethurst PA, de Groot PG, Raynal N, Farndale RW. Synergism between platelet collagen receptors defined using receptor-specific collagen-mimetic peptide substrata in flowing blood. Blood 115, 5069-5079 (2010).

38. Van Kruchten R, Cosemans JM, Heemskerk JW. Measurement of whole blood thrombus formation using parallel-plate flow chambers: a practical guide. Platelets 23, 229-242 (2012).

39. Heemskerk JW, Sakariassen KS, Zwaginga JJ, Brass LF, Jackson SP, Farndale RW. Collagen surfaces to measure thrombus formation under flow: possibilities for standardization. J Thromb Haemost 9, 856-858 (2011).

40. Bergmeier W, Oh-Hora M, McCarl CA, Roden RC, Bray PF, Feske S. R93W mutation in Orai1 causes impaired calcium influx in platelets. Blood 113, 675-678 (2009).

41. Nurden A, Nurden P. Advances in our understanding of the molecular basis of disorders of platelet function. J Thromb Haemost 9 (Suppl. 1), 76-91 (2011).

42. Schaff M, Tang C, Maurer E, Bourdon C, Receveur N, Eckly A, Hechler B, Arnold C, de Arcangelis, Nieswandt B, Denis CV, Lefebre O, Georges-Labouesse E, Gachet C, Lanza F, Magin PH. Integrin $a_{6} \beta_{1}$ is the main receptor for vascular laminins and plays a role in platelet adhesion, activation and arterial thrombosis. Circulation 128, 541-552 (2013).

43. Ozaki Y, Suzuki-Inoue K, Inoue O. Novel interactions in platelet biology: CLEC-2/podoplanin and laminin/GPVI. J Thromb Haemost 7 Suppl 1, 191-194 (2009).

44. Lisman T, Raynal N, Groeneveld D, Maddox B, Peachey AR, Huizinga EG, de Groot PG, Farndale RW. A single high-affinity binding site for von Willebrand factor in collagen III, identified using synthetic triple-helical peptides. Blood 108, 3753-3756 (2006).

45. Westein E, van der Meer AD, Kuijpers MJ, Frimat JP, van den Berg A, Heemskerk JW. Atherosclerotic geometries spatially confine and exacerbate pathological thrombus formation poststenosis in a von Willebrand factor-dependent manner. Proc Natl Acad Sci USA 110, 1357-1362 (2013).

46. Hooley E, Papagrigoriou E, Navdaev A, Pandey AV, Clemetson JM, Clemetson KJ, Emsley J. The crystal structure of the platelet activator aggretin reveals a novel $(a \beta)_{2}$ dimeric structure. Biochemistry 47, 7831-7837 (2008).

47. Knight CG, Morton LF, Onley DJ, Peachey AR, Messent AJ, Smethurst PA, Tuckwell DS, Farndale RW, Barnes MJ. Identification in collagen type I of an integrin $a_{2} \beta_{1}$-binding site containing an essential GER sequence. J Biol Chem 273, 33287-33294 (1998).

48. Smethurst PA, Joutsi-Korhonen L, O'Conner MN, Wilson E, Jennings NS, Garner SF, Zhang Y, Knight CG, Dafforn TR, Buckle A, Ijseldijk MJ, de Groot PG, Watkins NA, Farndale RW, Ouwehand WH. Identification of the primary collagen-binding surface on human glycoprotein VI by site-directed mutagenesis and by a blocking phage antibody. Blood 103, 903-911 (2004).

49. Cauwenberghs N, Vanhoorelbeke K, Vauterin S, Westra DF, Romo G, Huizinga EG, Lopez JA, Berndt MC, Harsfalvi J, Deckmyn H. Epitope mapping of inhibitory antibodies against platelet glycoprotein Iba reveals interaction between the leucine-rich repeat $\mathrm{N}$-terminal and $\mathrm{C}$-terminal flanking domains of 
glycoprotein Iba. Blood 98, 652-660 (2001).

50. Rosado JA, Meijer EM, Hamulyak K, Novakova I, Heemskerk JW, Sage SO. Fibrinogen binding to the integrin $\mathrm{a}_{\mathrm{IIb}} \beta_{3}$ modulates store-mediated calcium entry in human platelets. Blood 97, 2648-2656 (2001).

51. Feske S, Gwack Y, Prakriya M, Srikanth S, Puppel SH, Tanasa B, Hogan PG, Lweis RS, Daly M, Rao A. A mutation in Orai1 causes immune deficiency by abrogating CRAC channel function. Nature 441, 179-185 (2006).

52. Munnix IC, Kuijpers MJ, Auger J, Thomassen CM, Panizzi P, van Zandvoort MA, Rosing J, Bock PE, Watson SP, Heemkerk JW. Segregation of platelet aggregatory and procoagulant microdomains in thrombus formation: regulation by transient integrin activation. Arterioscler Thromb Vasc Biol 27, 2484-2490 (2007).

53. Cosemans JM, Schols SE, Stefanini L, de Witt SM, Feijge MA, Hamulyak K, Deckmyn H, Bergmeier W, Heemskerk JW. Key role of glycoprotein Ib/V/IX and von Willebrand factor in platelet activation-dependent fibrin formation at low shear flow. Blood 117, 651-660 (2011). 



\section{CHAPTER 5}

DISTINCT ROLE OF VON WILLEBRAND FACTOR TRIPLET BANDS IN GLYCOPROTEIN IB-DEPENDENT PLATELET ADHESION AND THROMBUS FORMATION UNDER FLOW

Fuchs B, de Witt SM, Solecka BA, Kröning M, Obser T, Cosemans JM, Schneppenheim R, Heemskerk JW, Kannicht C 


\begin{abstract}
Multimeric glycoprotein von Willebrand factor (VWF) exhibits a unique triplet structure of individual oligomers, resulting from ADAMTS-13 (a disintegrin and metalloproteinase with thrombospondin type 1 motifs- $\underline{13}$ ) cleavage. The faster and slower migrating triplet bands of a given VWF multimer respectively have one shorter or longer $N$-terminal peptide sequence. Within this peptide sequence, the A1 domain regulates interaction of VWF with platelet glycoprotein (GP)Ib. Therefore, platelet-adhesive properties of two VWF preparations with similar multimeric distribution but different triplet composition were investigated for differential functional activities. Preparation A was enriched in intermediate triplet bands, while preparation B predominantly contained larger triplet bands. Binding studies revealed that preparation A displayed a reduced affinity for recombinant GPIb, but an unchanged affinity for collagen type III, when compared to preparation B. Under high-shear flow conditions, preparation A was less active in recruiting platelets to collagen type III. Furthermore, when added to blood from patients with von Willebrand disease (VWD), defective thrombus formation was less restored. Thus, VWF forms lacking larger size triplet bands appear to have a decreased potential to recruit platelets to collagen-bound VWF under arterial flow conditions. By implication, changes in triplet band distribution observed in patients with VWD may result in altered platelet adhesion at high-shear flow.
\end{abstract}

\title{
Introduction
}

Hemostasis requires the combined action of blood platelets and vascular and plasmatic factors, with von Willebrand factor (VWF) having a key role. In blood, VWF circulates as a large glycoprotein consisting of multimers of heterogeneous sizes. At high-shear flow conditions, VWF mediates platelet adhesion via the glycoprotein (GP)Ib-V-IX complex, in particular by depositing to collagen fibres, at which it avidly binds. Collagen-bound VWF thus plays a critical role in platelet tethering, translocation, and stable adhesion at arterial flow conditions above a critical shear rate of $500-1000 \mathrm{~s}^{-11,2}$. VWF binds to collagen types I and III via its A3 domain, whereas binding to type VI collagen has been attributed to VWF A1 domain ${ }^{3-5}$. In addition, VWF is implicated in platelet GPIb-dependent pro-coagulant activity and fibrin formation ${ }^{6}$, and it protects factor VIII from rapid proteolytic inactivation ?.

It is well established that large VWF multimers, which are secreted from endothelial cells, are cleaved into smaller forms by the metalloproteinase ADAMTS-13 (a disintegrin and metalloproteinase with thrombospondin type 1 motifs $\underline{13}$ ). This protease cleaves the $\mathrm{Y}^{1605}-\mathrm{M}^{1606}$ peptidyl bond within the $A 2$ domains of VWF multimers, thus generating smaller size multimers of VWF 8. Because of asymmetric cleavage in the VWF A2 domain, ADAMTS-13 degradation results in larger and smaller size monomeric forms of $176 \mathrm{kDa}$ ( $C$-terminal peptide sequence) and $140 \mathrm{kDa}$ ( $\mathrm{N}$-terminal peptide sequence), respectively (Figure $1 \mathrm{~A}$ ). On agarose gels these represent the two satellite bands, flanking the major band of all VWF multimers ${ }^{9,10}$ forming a so-called VWF triplet. The slower and faster migrating triplet bands thus either contain or lack one $140 \mathrm{kDa} N$-terminal peptide sequence compared to the intermediate VWF triplet band, respectively ${ }^{11}$. The variable part of individual VWF triplets resides in the $N$-terminal protein part comprising the A1 domain, which contains binding sites for heparin, coagulation factor VIII and GPIb and, hence, is highly relevant to VWF function.

Because the properties of individual VWF triplet bands have not been investigated so far, we aimed to separate normal plasma-derived VWF into sub-fractions with a similar multimeric composition, but different triplet band patterns. Therefore, we made use of the property that the longer-chain, slower migrating triplet bands of VWF multimers containing one additional $\mathrm{N}$-terminal $140 \mathrm{kDa}$ peptide sequence more avidly bind to heparin ${ }^{12}$. We used heparin affinity column separation, followed by size exclusion chromatography to obtain VWF preparations with a similar multimer profile, but distinct pattern of triplet bands, resulting in different affinities to GPIb and different capabilities to promote GPIb-dependent platelet adhesion under high-shear flow conditions. 


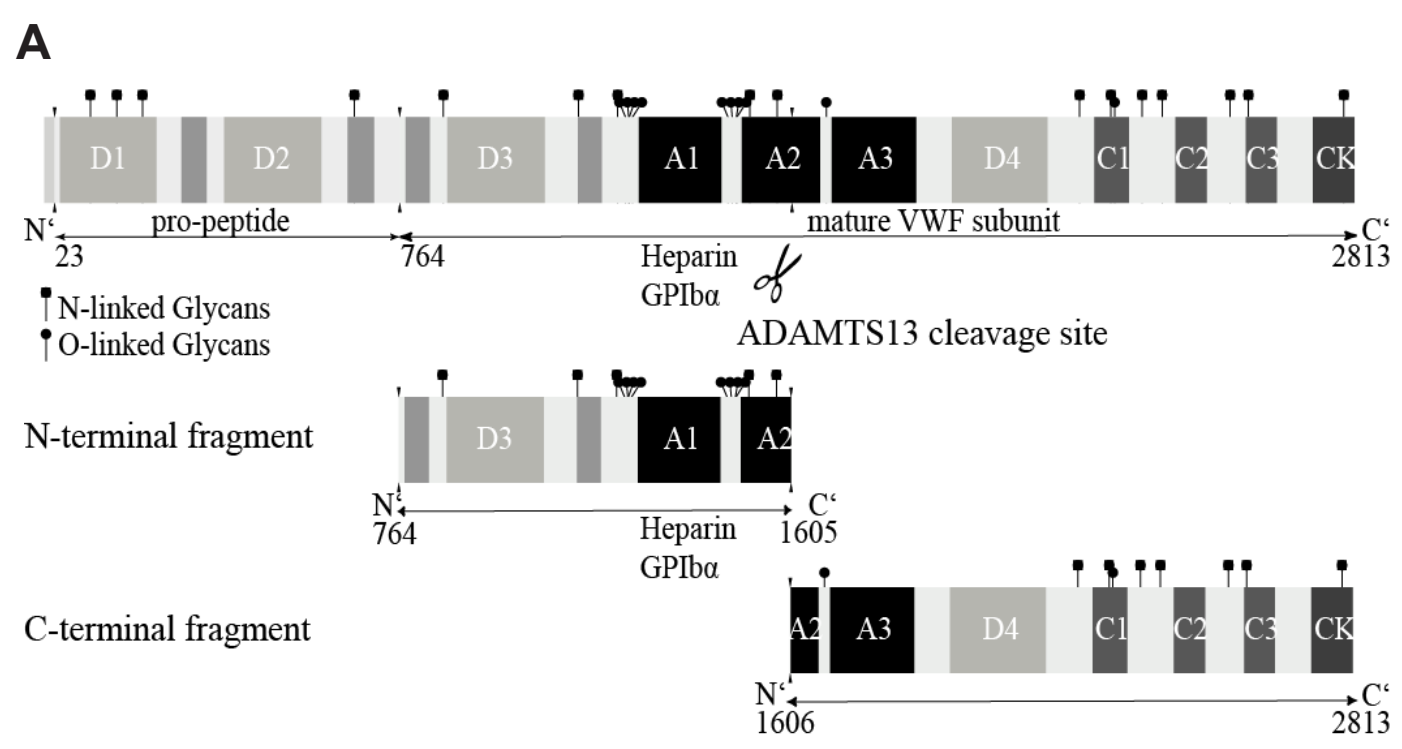

B

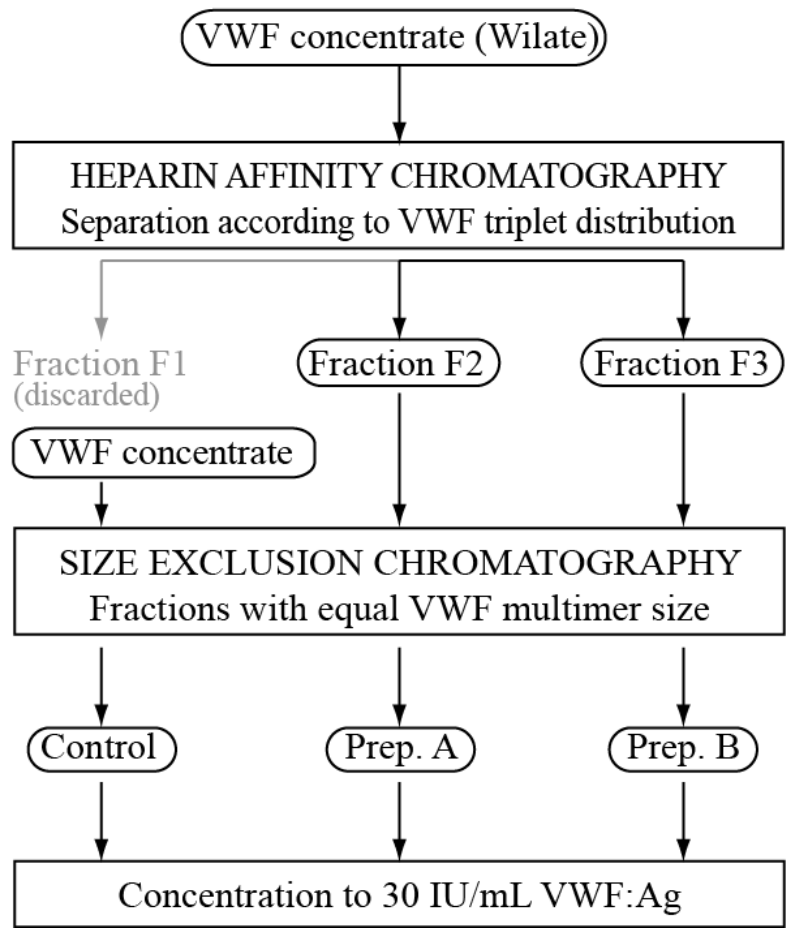

Figure 1. Domain structure of VWF and flow-chart to obtain preparations with different VWF triplet distribution. (A) VWF domains indicating binding sites for heparin and GPIb in the A1 domain. The pro-peptide of AA 23-763 after cleavage of the pre-pro-peptide is depicted below the domain structure as well as the mature VWF protein starting with AA 764-2813 (numbering according to the pre-pro-protein). Glycosylation is indicated as squares ( $\mathrm{N}$-linked glycans) and circles (O-linked glycans). ADAMTS-13 cleavage site in the A2 domain is indicated as well. This cleavage results in a $140 \mathrm{kDa} N$-terminal fragment comprising the D3-A1 domains and part of the A2 domain with binding sites for e.g. GPIb and heparin. Cleavage also results in a larger $176 \mathrm{kDa}$ C-terminal fragment, comprising part of the A2 domain as well as VWF domains A3D4C1C2C3CK. Information source: P04275, UniProtKB/Swiss-Prot database. (B) Flow-chart to separate plasma-derived VWF into preparations with different triplet composition, but similar multimeric composition. Application of desalted VWF concentrate (Wilate) on heparin affinity chromatography column results in three fractions, fraction F1 (discarded) and fractions F2 and F3 with distinct triplet composition. By using size exclusion chromatography, F2 and F3 are resolved in sub-fractions of similar molecular weight ranges and, hence, similar multimeric composition, giving preparations A and B, respectively. Desalted whole VWF concentrate is also subjected to size exclusion chromatography to obtain a control preparation with similar molecular weight ranges to preparations $A$ and $B$. 
A

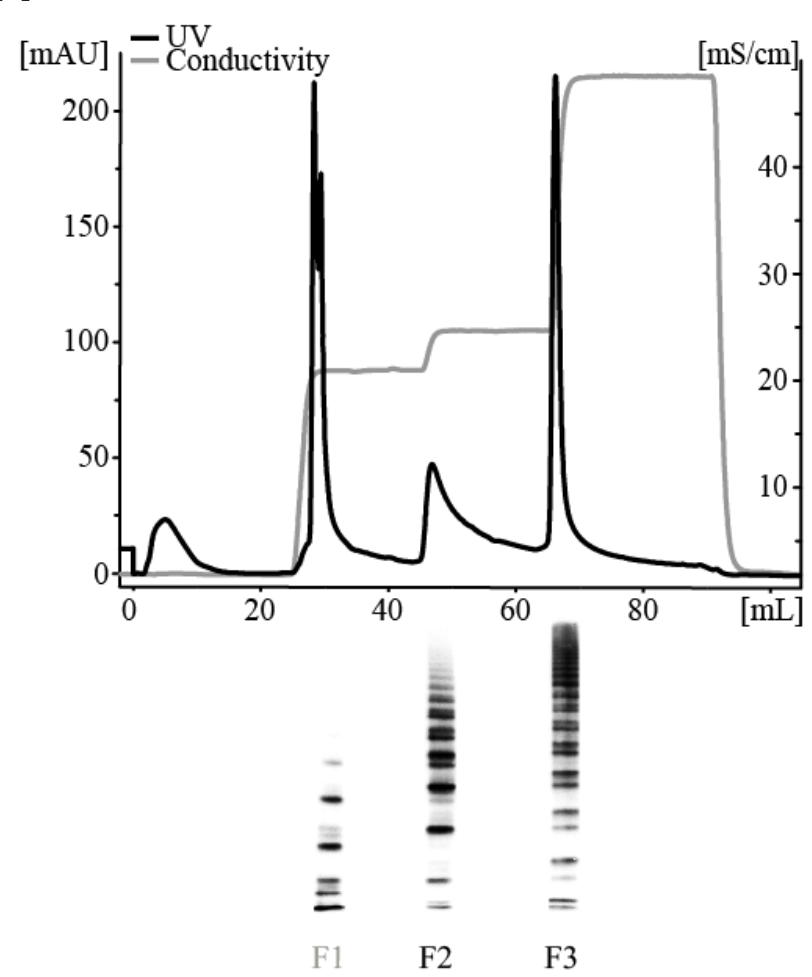

B

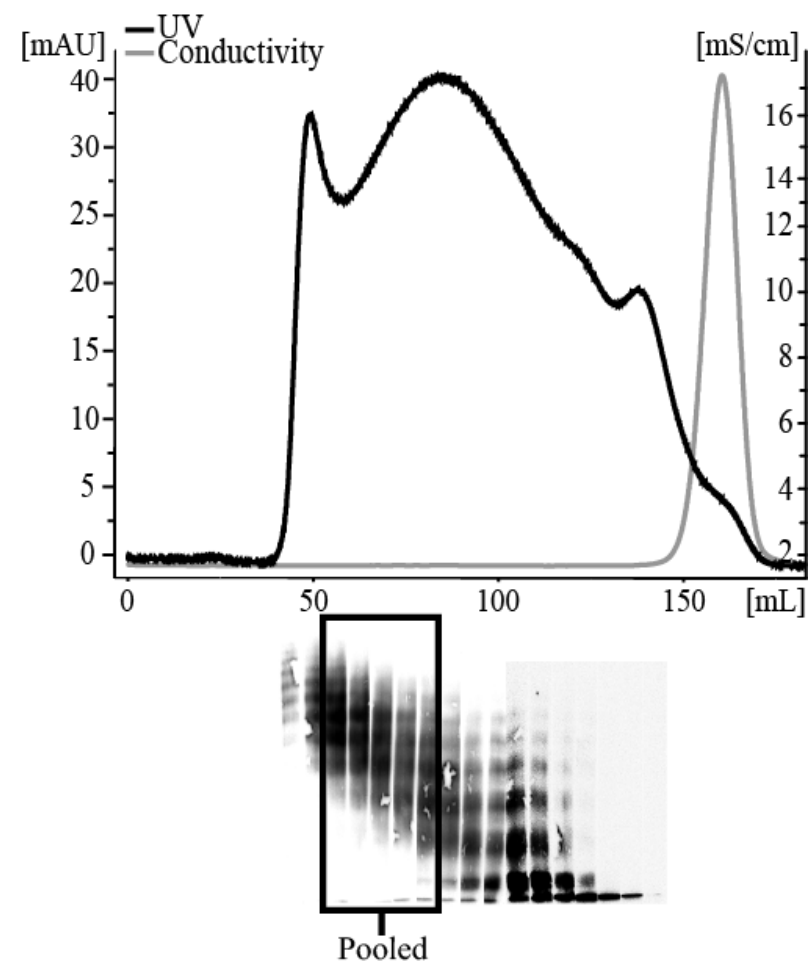

Figure 2: Obtaining VWF preparations with different triplet forms by heparin affinity and size exclusion chromatography. (A) Plasma-derived VWF concentrate was applied to a heparin affinity column, and eluted step-wise with 190, 230 and $500 \mathrm{mM} \mathrm{NaCl}$ (gray line of eluent conductivity). Protein peaks were collected as fractions 1-3 (F1-3), and subjected to Western blotting (1.6\% agarose gels). Note differences in multimeric and triplet band composition of the fractions. F1 containing mostly small VWF multimers with little biological activity was discarded. F2 predominantly contained the medium to large VWF multimers with only intermediate triplet bands, while F3 consisted of more of the larger VWF multimers enriched in slower migrating triplet bands. (B) Size exclusion chromatography of unseparated VWF concentrate, resulting in initial elution of larger VWF multimers. Western blots of $1.2 \%$ agarose gels illustrate the multimeric sizes of individual fractions, and pooling of fractions to obtain the control VWF preparation. Note that preparations $A$ and $B$ were obtained using the same size exclusion chromatography as shown for the control in Figure $2 \mathrm{~B}$, but starting from fractions F2 and F3, respectively (not shown in this figure)

\section{Materials and methods}

\section{Materials}

Plasma-derived VWF/factor VIII concentrate Wilate (lot no. A925A189) used for fractionation was from Octapharma (Vienna, Austria). First international standard VWF concentrate 00/514 (NIBSC, Hertfordshire, UK) was used as reference for quantification. VWF levels are given as antigen concentrations, unless indicated otherwise. Standard normal human plasma (SHP) was from Haemochrom Diagnostica (Essen, Germany). Collagen type III from human placental villi was obtained from Biozol Diagnostica (Eching, Germany). F(ab)2 fragments of monoclonal antibody (Ab) 6B4 against the VWF-binding epitope on GPIba was a kind gift of Dr. H. Deckmyn ${ }^{13}$. H-Phe-Pro-Arg chloromethyl ketone (PPACK) came from Calbiochem (La Jolla, CA, USA).

\section{Isolation of VWF preparations with different triplet composition}

Plasma-derived VWF concentrate (Wilate) was desalted using a PD-10 column and eluted with 20 mM Tris, pH 7.4 (GE Healthcare, Uppsala, Sweden). The VWF concentrate was then fractionated according to triplet structure by fast protein liquid chromatography (FPLC), employing a $5 \mathrm{~mL}$ HiTrap heparin HP affinity column (GE Healthcare) and stepwise gradient elution with 190, 230 and $500 \mathrm{mM} \mathrm{NaCl}$ in $20 \mathrm{mM}$ Tris, pH 7.4 (Figure 1B). Of the three fractions (F1-3), only F2 and 
F3, obtained at 230 and $500 \mathrm{mM} \mathrm{NaCl}$ elution, respectively, were used for further purification. The other fraction F1 was enriched in the faster migrating triplet band, but contained only small VWF multimers with low biologic activity (Figure 2A), and was hence discarded.

Each of the fractions F2 and F3 (Figure 2A), as well as the unfractionated Wilate (original sample $=$ control preparation) were differentially separated into sub-fractions by FPLC using a Sepharose CL2B size exclusion column. Elution was performed with Tris-buffer, pH 7.4 (20 mM Tris, $0.02 \% \mathrm{v} / \mathrm{v}$ Tween 20 ). Sub-fractions from this column for each starting sample (i.e., fractions $F 2$ and F3, and control preparation) were then separately pooled to yield different VWF preparations with a similar multimeric distribution. This was done for the control preparation (exemplary shown in Figure 2B), as well as for fractions F2 and F3 (not shown but similar process to that shown for control preparation in Fig 2B) to give preparations A and B, respectively (see Fig 1B). Each separate column fraction pool was then concentrated using an Amicon Ultra-4 centrifugal filter device (100K; Millipore, Billarica, MA, USA) at $2000 \mathrm{~g}$ to a VWF:Ag level of about $30 \mathrm{IU} / \mathrm{mL}$. Fraction analysis for VWF multimer distribution was by $1.2 \%$ agarose gel electrophoresis; and analysis for VWF triplet composition was by $1.6 \%$ agarose gel electrophoresis, followed by Western blotting ${ }^{15}$. Densitometric analysis of blots was performed with Multi Gauge V3.2 software (Fujifilm, Düsseldorf, Germany).

VWF preparations were assessed for collagen binding using a VWF:CB ELISA (TECHNOZYM ${ }^{\circledR}$ VWF: CBA ELISA Kit, Technoclone, Vienna, Austria). VWF ristocetin cofactor activity (VWF:RCo) was determined using a BCS XP system (Siemens Healthcare, Eschborn, Germany); all values were calibrated against standard human plasma.

\section{ELISA assays}

Antigen concentrations of VWF were determined with a sandwich ELISA with polyclonal anti-human VWF antibody (Ab) (A0082, Dako, Hamburg, Germany) for capturing, followed by staining with horseradish peroxidase (HRP)-conjugated anti-human VWF Ab (P0226, Dako). Sigma Fast OPD tablets were used as chromogenic substrate (order no. P9187; Sigma-Aldrich, Schwerte, Germany), colour development was terminated with $1 \mathrm{M} \mathrm{HCl}$, and absorbance was measured at $492 \mathrm{~nm}$.

Binding of VWF fractions to platelet-derived GPIb was determined with an immuno-assay employing recombinant, FLAG-tagged GPIba fragment with two gain-of-function mutations (G233V and M239V), enabling VWF binding in the absence of ristocetin ${ }^{16-18}$. The rGPIba fragment was immobilized via its FLAG tag in 96-well plates pre-coated with anti-FLAG Ab (F3165, Sigma-Aldrich). Bound VWF was measured using a polyclonal anti-human VWF Ab and a HRP-conjugated secondary Ab (P0226 and A0082, Dako) in combination with TMB substrate solution (T0565, SigmaAldrich). The colouring reaction was terminated with $0.5 \mathrm{M} \mathrm{H}_{2} \mathrm{SO}_{4}$, and absorbance was read at $450 \mathrm{~nm}$.

\section{Surface plasmon resonance}

Binding of VWF preparations to collagen type III was determined by surface plasmon resonance (SPR) using a Biacore 2000 (GE Healthcare), CM5 chips and amine coupling chemistry, basically as described ${ }^{19}$. In brief, collagen type III was immobilized on the chip surface at an amount of 800-1000 response units (RU). VWF samples were injected at increasing concentrations $(0.2,0.4$, 1.3 and $4 \mathrm{IU} / \mathrm{mL}$ ) in HBS-EP running buffer (3.4 mM EDTA, $10 \mathrm{mM}$ HEPES, $150 \mathrm{mM} \mathrm{NaCl}, 0.005 \%$ Tween 20; pH 7.4) at a flow rate of $20 \mu \mathrm{L} / \mathrm{min}$. A chip with immobilized polyclonal rabbit anti-human collagen I-V Ab (Kamiya Biomedical, Seattle, USA) served as reference for unspecific binding. Chips were regenerated after each run ${ }^{20}$.

For determination of rGPIb binding kinetics by SPR, purified gain-of-function rGPIba fragment carrying a His-tag was immobilized onto NTA-chips (GE Healthcare) at 1000 RU. Samples of VWF were injected at increasing concentrations of $0.3,0.9,2.8$ and $8.4 \mathrm{IU} / \mathrm{mL}$. Analysis of all SPR binding curves was done with BiaEvaluation software (GE Healthcare), assuming Langmuir 1:1 
binding kinetics and a molecular weight of the VWF monomer of $270 \mathrm{kDa}$.

\section{Platelet adhesion and thrombus formation under flow}

Leukocyte-depleted platelet concentrates collected by aphaeresis and red cell concentrates were obtained from Haema (Berlin, Germany). These concentrates were used to prepare washed platelets and red cells, respectively. Shear- and VWF-dependent platelet adhesion to collagen type III using reconstituted blood was performed, as described elsewhere ${ }^{21}$. Briefly, $2.5 \times 10^{8}$ platelets $/ \mathrm{mL}$ were labelled with 5-chloromethylfluorescein diacetate (CMFDA, Invitrogen, Karlsruhe, Germany), and reconstituted with washed red cells (hematocrit of $40 \%$ ) and VWF preparations (1 IU/mL). Viscosity of the reconstituted blood samples was measured with an AMVn falling sphere viscometer (Anton Paar, Ostfildern, Germany) to ensure a dynamic viscosity of $\sim 3 \mathrm{mPa} \cdot \mathrm{s}$. Samples were per-

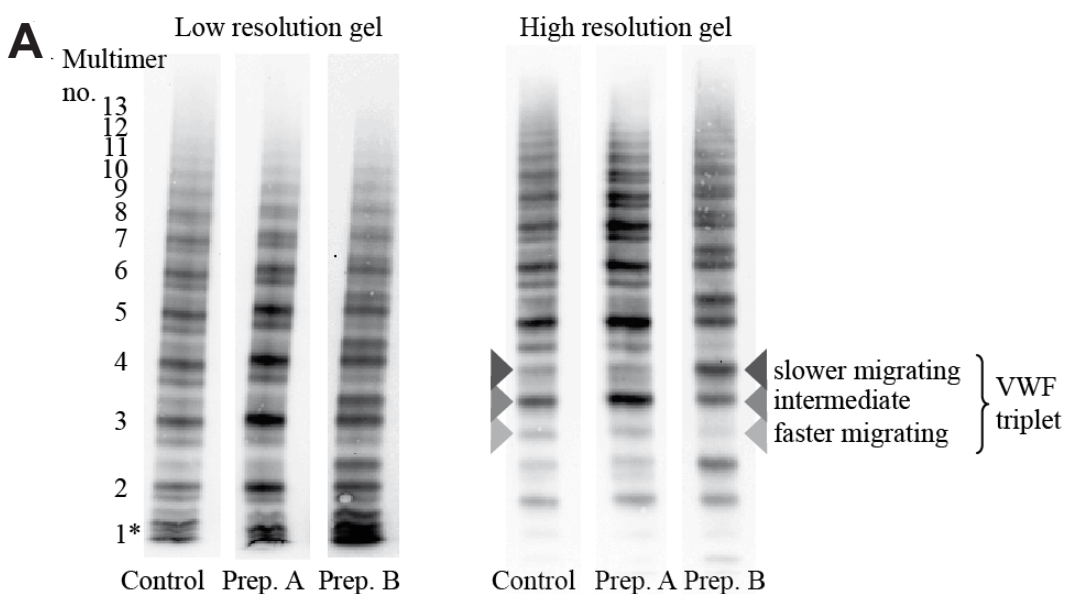

B

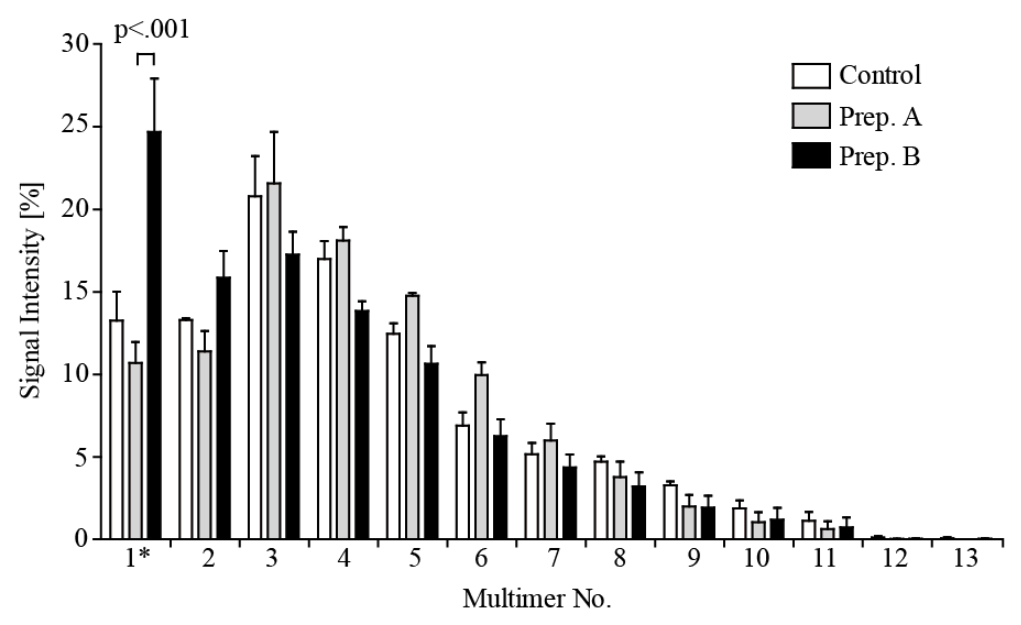

C

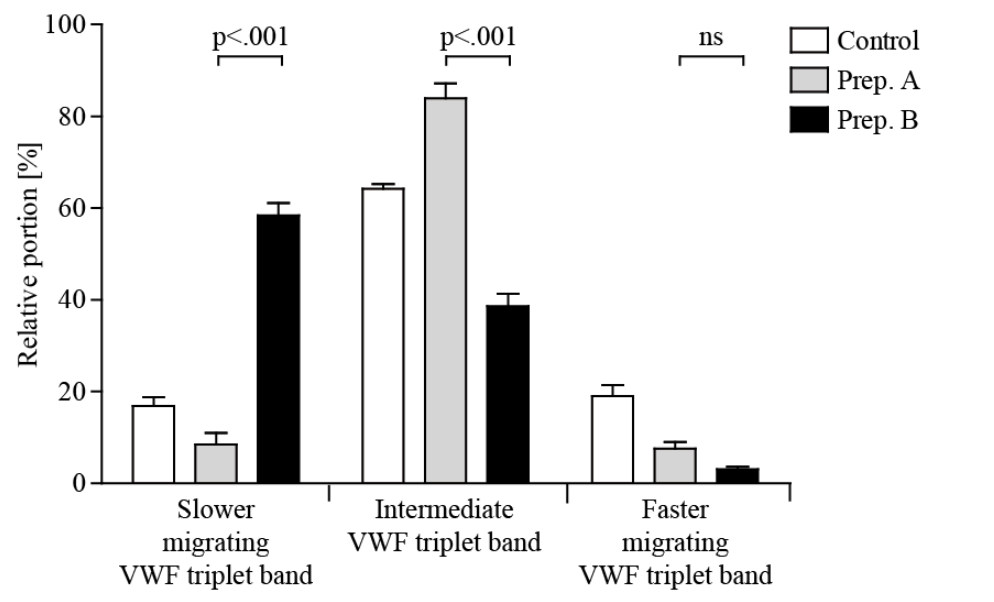

Figure 3. Triplet and multimer composition of fractionated VWF preparations. Comparison of control preparation with normal triplet structure (VWF concentrate subjected to size exclusion chromatography), and preparations $\mathrm{A}$ and $\mathrm{B}$ (separated by heparin affinity chromatography and then size exclusion chromatography) analysed by agarose gel electrophoresis and Western blotting. (A) Representative Western blots. Left: low-resolution $1.2 \%$ agarose gels, showing multimer sizes from $1 \mathrm{mer}$ to 13 mer (*1mer band plus dye front). Right: high-resolution $1.6 \%$ agarose gels, showing triplet composition of multimers with faster, intermediate and slower migrating triplet bands (arrowheads). Note that preparations A and B, and the control preparation were similar in multimer composition (2-13mer). (B) Relative distribution of VWF 1-13mer multimers of control preparation and preparations $A$ and B. (C) Proportions of each of three triplet bands in control preparation and preparations $A$ and $B$. Means \pm SEM $(n=4)$. Note here the difference in triplet bands: Preparation A was enriched in intermediate triplet bands, while preparation $\mathrm{B}$ predominantly contained larger triplet bands. 
fused at a shear rate of $1700 \mathrm{~s}^{-1}$ through a flow chamber coated with collagen type III ( $\mu$-slide VI, Ibidi, Munich, Germany). Platelet adhesion was monitored in real time for up to $8 \mathrm{~min}$. After perfusion and fixation, 24 randomly chosen images in the centre of the flow channel were recorded, and analysed using AxioVision 4.6 software (Carl Zeiss, Göttingen, Germany).

The VWF preparations were also tested in whole-blood perfusion assays, using a miniaturized parallel-plate flow chamber and equipment as described before 22,23 . Chamber surfaces were coated with collagen type III $(0.1 \mathrm{mg} / \mathrm{mL})$, and blocked with HEPES buffer, $\mathrm{pH} 7.45$ plus $1 \%$ BSA. Experiments were carried out with blood from healthy controls and from two patients with VWD. A patient with type 1 VWD had a VWF:RCo activity of $29 \%$. A patient with type 2 A VWD had a VWF: RCo activity of $<10 \%$, a VWF antigen level of $25-35 \%$, and a factor VIII level of $30 \%$; multimer analysis revealed that the highest multimers were lacking, which is compatible with type $2 \mathrm{~A}$.

Blood was drawn into PPACK anticoagulant, as described previously ${ }^{24}$. This direct thrombin active-site inhibitor achieves complete anticoagulation, while maintaining physiological concentrations of free $\mathrm{Mg}^{2+}$ and $\mathrm{Ca}^{2+}$ ions. Blood samples were spiked with vehicle or VWF preparations at final concentrations of $2 \mathrm{IU} / \mathrm{mL}$. The blood was then perfused through the flow chamber at a shear rate of $1700 \mathrm{~s}^{-1}$ for $4 \mathrm{~min}$. Four to five randomly chosen phase-contrast images were captured with a digital CCD camera at the end of perfusion. Images were analysed as described above. Blood donors had given full informed consent, and not taken medication interfering with platelet function for at least two weeks.

\section{Statistical analyses}

Significance of data was analysed with GraphPad Prism version 5.01 (GraphPad Software, San Diego, CA, USA). Normal distribution was confirmed using the Kolmogorov-Smirnov normality test. Differences between grouped data were compared with an unpaired Student's $t$-test. Data from gels with VWF multimers and SPR analyses were compared by 2 way ANOVA, using Bonferroni post-testing to compare replicates.

\section{Results}

Separation of plasma-derived VWF by heparin affinity chromatography (Figure 1) resulted in two fractions (F2 and F3) with markedly differences in triplet structure, but also to some extent in multimeric composition (Figure 2A). Each of the fractions, as well as the original VWF preparation (used as a control) was further separated by size exclusion chromatography, and sub-fractions were pooled to obtain preparations with equal multimeric distribution (process shown in Figure $2 B$ exemplary for the control).

The resulting fractionated VWF pools, preparation A and B, as well as the (unfractionated) control preparation, were characterized by electrophoresis on low- and high-resolution agarose gels and Western blotting (Figure 3A). Densitometric analysis of blots showed that, as aimed for, preparations $A$ and $B$ were similar in multimer composition (2-13mer), as was the control preparation (Figure $3 \mathrm{~B}, \mathrm{p}>0.05$ ). A significant difference in the preparations was only seen in the 1-mer composition, but this was not expected to influence subsequent findings. Importantly, the various preparations displayed marked and significant differences with regard to triplet band composition (Figure 3C). Preparation B was greatly enriched in the slower migrating triplet bands of essentially all multimers at the expense of intermediate triplet bands; in comparison, preparation $A$ was greatly reduced in the slower migrating triplet bands and contained larger proportions of intermediate triplet bands $(\mathrm{p}<0.001)$. Accordingly, the two-column separation had resulted in two VWF fractions with prominent differences in presence of larger size triplet bands, but with similar multimeric composition.

The original VWF concentrate as well as the various preparations were concentrated to obtain solutions with the same VWF antigen concentrations, and further characterized using conventional VWF assays, namely VWF:CB and VWF:RCo. VWF:CB to VWF:Ag ratios were: $1.21 \pm 0.10$ (original 
A
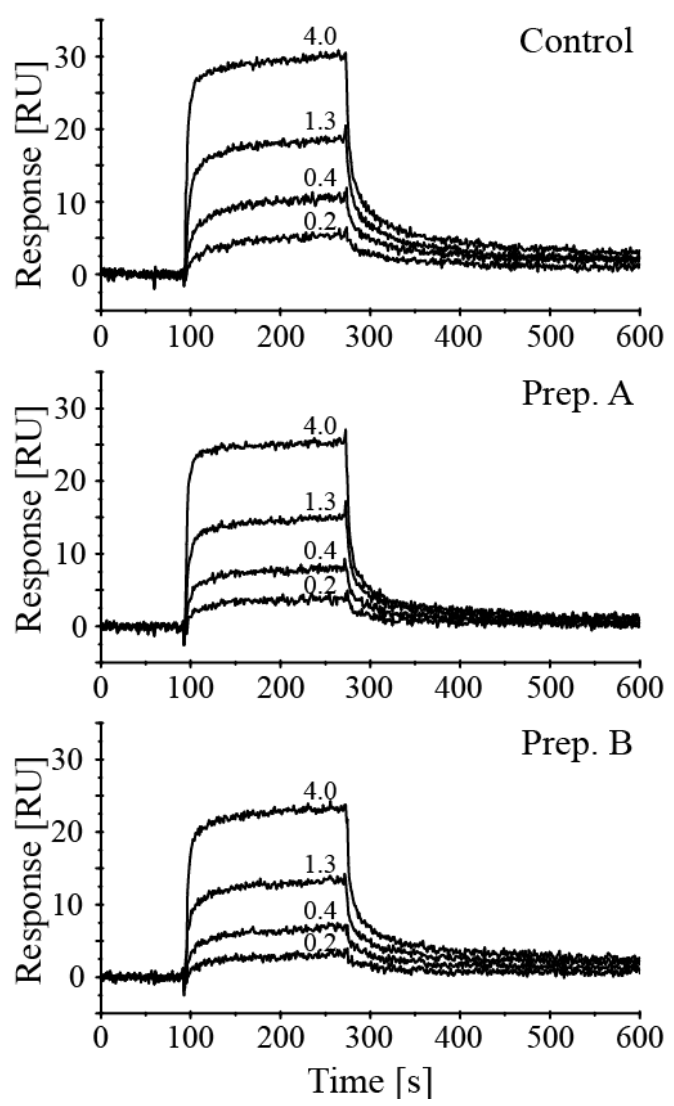

B

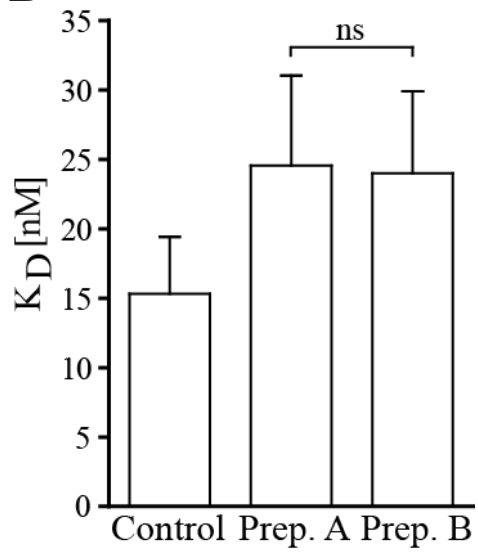

Figure 4. Binding of VWF preparations to collagen. Binding kinetics of VWF preparations to collagen type III were established by surface plasmon resonance measurements. (A) Representative binding curves with increasing concentrations of $0.2,0.4,1.3$ and $4 \mathrm{IU} / \mathrm{mL}$ for control preparation and for preparations $A$ and $B$, as indicated. Binding is expressed as response units (RU). (B) Calculated affinity constant values $\left(K_{D}\right)$ of various VWF preparations. Means \pm SEM $(n=6)$.

sample), 0.91 \pm 0.02 , (control), $0.81 \pm 0.02$ (Prep. A) and 0.78 \pm 0.01 (Prep. B); VWF:RCo to VWF: Ag ratios were $0.41 \pm 0.04$ (original sample), $0.37 \pm 0.01$ (control), $0.37 \pm 0.01$ (Prep. A), $0.42 \pm 0.01$ (Prep. B). Hence, in comparison to the original VWD concentrate, the columnseparated preparations were slightly reduced in collagen binding and ristocetin cofactor activity, most likely due to partial retaining of the highest multimers on the heparin column (see below). Notably, preparation B had higher VWF:RCo but similar VWF:CB compared to preparation A.

Using SPR, we analysed binding properties of the three preparations to surface-immobilized collagen type III. Preparations A and B gave similar binding curves, when applied at increasing concentrations of 0.2 to $4 \mathrm{IU} / \mathrm{mL}$ (Figure $4 \mathrm{~A}$ ). Determination of the affinity constants, assuming conventional Langmuir kinetics, resulted in similar dissociation equilibrium constant $\left(K_{D}\right)$ values of 15-25 nM (Figure 4B), indicating no significant difference in binding affinity of the two preparations to collagen type III $(p=0.95)$. Both preparations displayed a tendency to diminished collagen binding in comparison to the unseparated control preparation.

Using a newly established method, a gain-of-function rGPIb fragment was immobilized to Biacore chips for investigating binding of the preparations to the platelet GPIb receptor. Binding curves were generated after injection of increasing concentrations of the VWF preparations (Figure $5 \mathrm{~A}$ ). Strikingly, the binding affinity of preparation $A$ in this case was significantly lower than of preparation $B$ (Figure $5 B, p=0.0014$ ). This lower affinity was due to a slower association rate in combination with a faster dissociation rate. Furthermore, at equal antigen concentrations, the maximal binding was on average $30 \%$ lower for preparation $A$ than for preparation $B$, likely as a consequence of the lower affinity. 
A

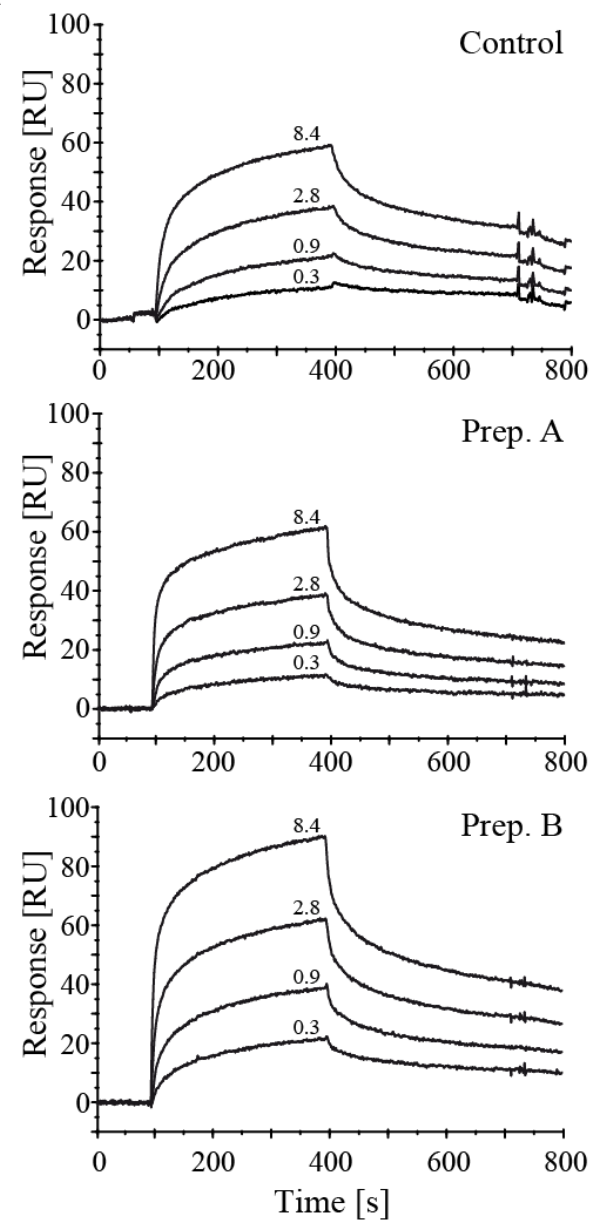

B
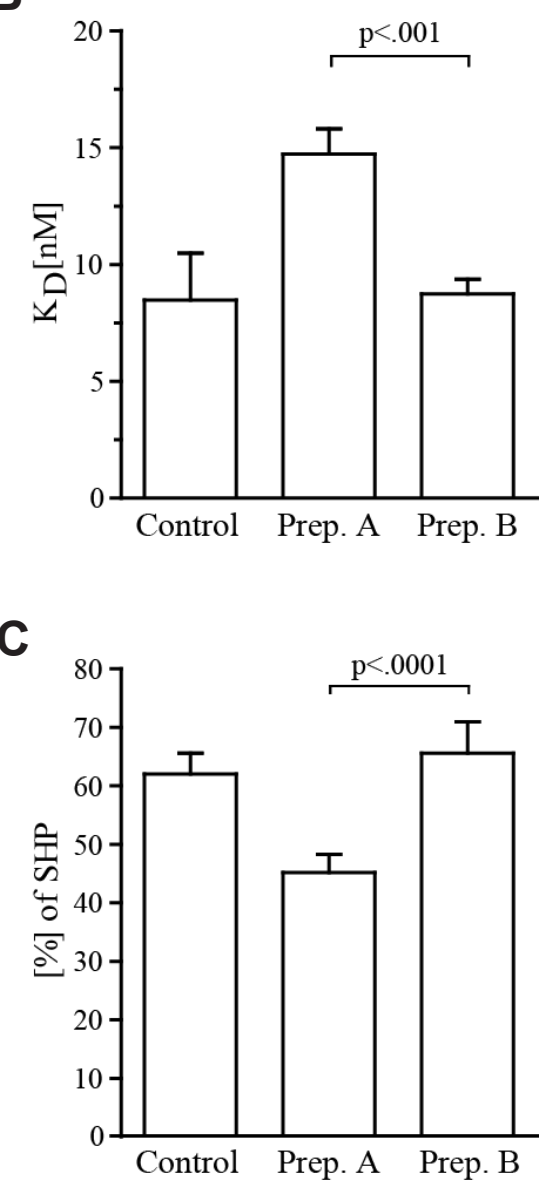

Figure 5. Binding of VWF preparations to recombinant GPIb. Surface plasmon resonance measurements to assess binding of VWF preparations to a gain-of-function rGPIb fragment. (A) Representative binding curves, expressed as response units (RU) at increasing concentrations of $0.3,0.9,2.8$ and $8.4 \mathrm{IU} / \mathrm{mL}$ for control and preparations $\mathrm{A}$ and B. (B) Corresponding $K_{D}$ values. (C) Binding of VWF preparations to rGPIb obtained with ELISA. Data are expressed as percentage of binding seen in standard human plasma (SHP). Means $\pm \operatorname{SEM}(n=5)$.

To confirm these differences in binding to GPIb, we also used an ELISA setup, where the same gain-of-function rGPIb fragment was immobilized on well plates. In this immuno-based assay, preparation A again showed a lower binding to GPIb than preparation B (Figure $5 C, p<0.0001$ ), such in agreement with the results obtained by SPR.

For functional analyses, we then investigated the ability of the VWF preparations to support GPIb-dependent platelet adhesion to type III collagen at high shear flow conditions. In the first set of experiments, reconstituted blood samples containing platelets (CMFDA-labelled) and red blood cells were supplemented with the control VWF preparation or with preparation A or B at 1 IU VWF/mL. After perfusion over collagen, the coverage of adhered platelets was determined by fluorescence microscopy. In this flow assay, preparation A was significantly less active in supporting platelet adhesion than preparation $B$ (Figure $6, p<0.0001$ ). To demonstrate the importance of VWF-GPIb interaction, similar flow experiments were performed in the presence of 6B4 mAb, directed against the VWF binding site of GPIb ${ }^{25}$. As indicated in Figure 6B, this antibody completely annulled the platelet-adhesive effect of the most active preparation $B$ (Figure $6 B, p<0.0001$ ).

In a second range of experiments, we compared the effects of addition of a re-combined F1-F3 preparation with that of the original VWF concentrate. The combination of F1, F2 and F3 gave a multimeric distribution pattern similar to that of the VWF preparations A/B (Suppl. Figure $\mathrm{S} 1$ ). Addition of either preparation (2 IU VWF/mL, final) to the reconstituted blood resulted in a 
similar increase in platelet deposition on collagen as seen with the combination of preparations $A$ and B (Suppl. Figure S2). This indicated that most of the biological activity of VWF was retained in the preparations $\mathrm{A}$ and $\mathrm{B}$.

In addition, we performed perfusion studies with whole blood from VWD patients containing reduced baseline levels of VWF. Blood was thus obtained from a patient with VWD type 1 (29\% VWF:RCo activity) and a patient with VWD type $2 A$ ( $<10 \%$ VWF:RCo activity). Samples of blood were spiked with the control preparation or with preparation A or B. After 4 min perfusion at 1700 $\mathrm{S}^{-1}$ shear rate, platelet deposition in thrombi was determined by enhanced contrast video microscopy. As shown in Figure 7, supplementation of all VWF preparations (control, A or B) at $2 \mathrm{IU} / \mathrm{mL}$ VWF resulted in a significant increase in platelet deposition with both type 1 and type 2A patient blood $(p<0.05)$. For the type 1 VWD patient, no significant difference was seen between preparations $A$ and B. However, for the type 2A VWD patient, preparation A was significantly less effective than preparation $B$ in stimulating thrombus formation $(p=0.0133)$. Further control experiments showed that none of the preparations modified platelet deposition, when added to blood from subjects with normal VWF levels (data not shown). Together, these findings suggest that VWF triplets lacking an additional $140 \mathrm{kDa} N$-terminal peptide sequence with the VWF A1 domain containing a GPIb binding site, are decreased in binding affinity to platelet GPIb, and are less active in supporting platelet thrombus formation on collagen type III under high-shear flow conditions.

\section{Discussion}

Distinct cleavage of VWF multimers in the A2 domain by the metalloproteinase ADAMTS- 13 results in VWF forms of various multimeric sizes, but with flanking satellite bands (so-called triplet bands) $10-12,26,27$. The slower migrating triplet bands of VWF multimers thereby contain an additional 140 kDa $\mathrm{N}$-terminal peptide fragment, whereas this $\mathrm{N}$-terminal part is missing in the faster migrating triplets, when compared to the respective intermediate VWF triplet bands ${ }^{11}$. Importantly, the $\mathrm{N}$-terminal part of VWF monomers that is cleaved off by ADAMTS-13 proteolysis comprises the D1D2D3A1 domains and part of the A2 domain. This part is known to be involved in the binding of VWF to coagulation factor VIII, heparin, collagen and platelet GPIb (reviewed by ${ }^{28}$ ).

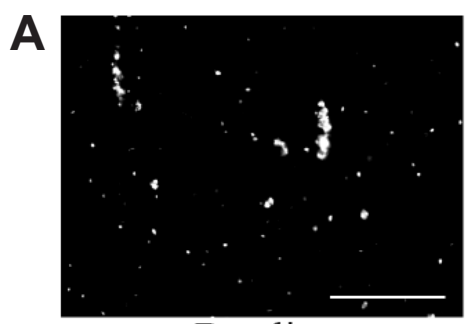

Baseline

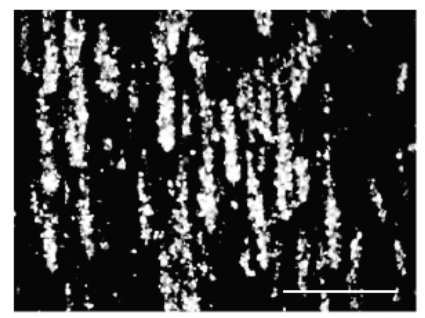

+ Prep. A

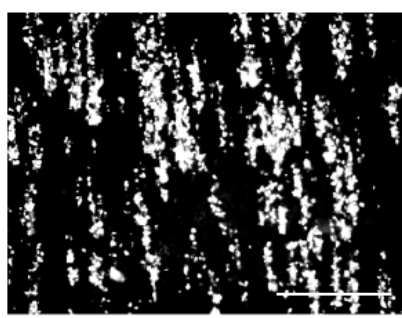

+ Control

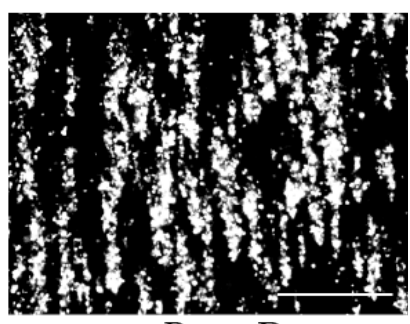

+ Prep. B
B

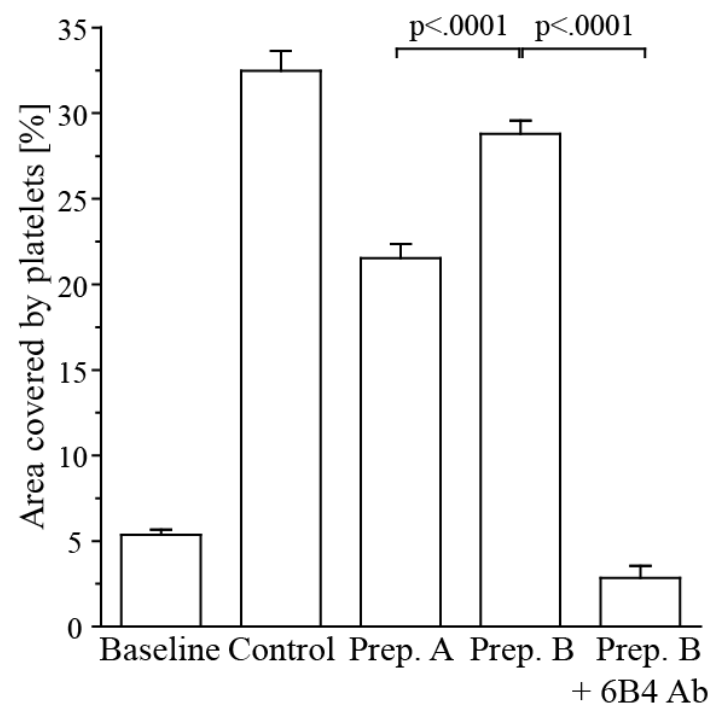

Figure 6. Effect of VWF preparations on shear- dependent platelet adhesion in reconstituted blood. Flow chamber experiments performed with reconstituted blood containing CMFDA-labelled platelets $(2.5 \times 108 / \mathrm{mL})$ and washed red blood cells (40\% hematocrit), flowed over a type III collagen surface at $1700 \mathrm{~s}-1$ shear rate. Reconstituted blood samples were supplemented with control VWF preparation or preparation A or B (1 IU/mL). The $6 \mathrm{~B} 4 \mathrm{mAb}(20 \mu \mathrm{g} /$ $\mathrm{mL}$ ) against the VWF binding site of GPIb was added, as indicated. Samples without VWF (baseline) served as negative control. (A) Representative fluorescence images (bars, $50 \mu \mathrm{m}$ ) after 8 min perfusion. (B) Analyzed surface area coverage of fluorescence after 8 min perfusion. Means \pm SEM ( $n=6 ; 3$ different platelet preparations). 
A

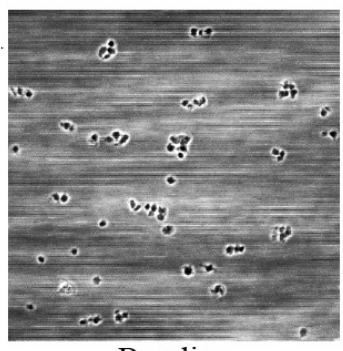

Baseline

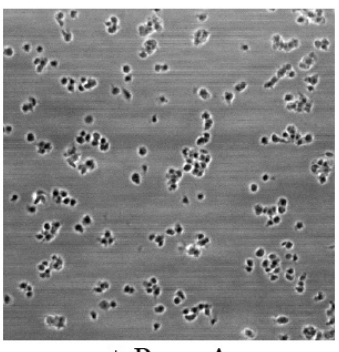

+ Prep. A

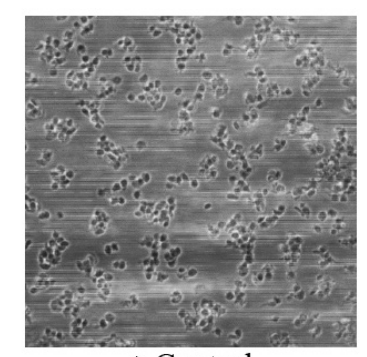

+ Control

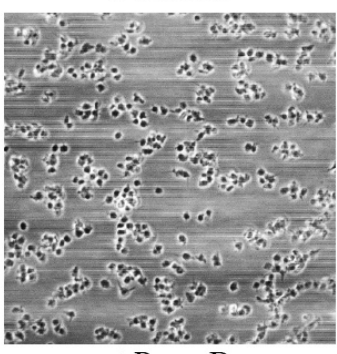

+ Prep. B
B

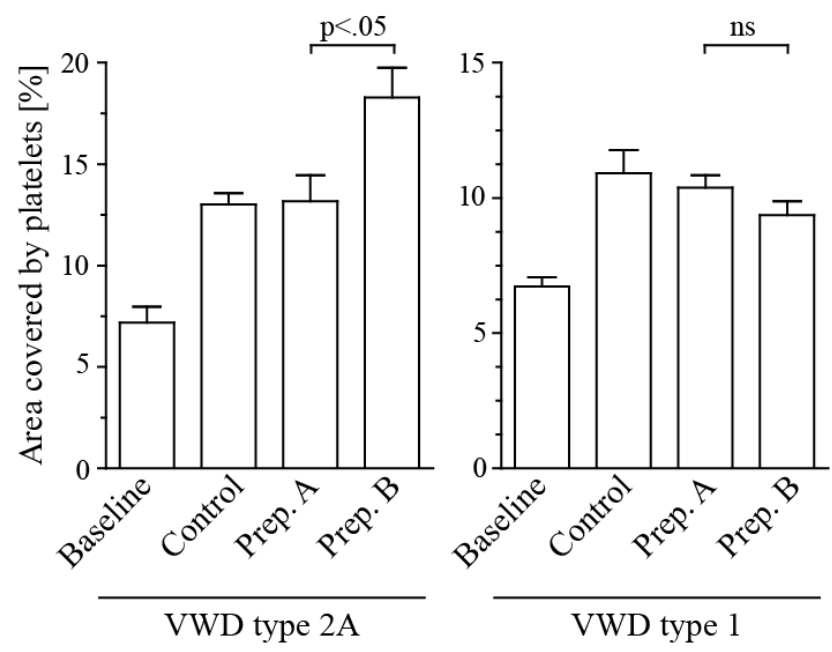

Figure 7. Effect of VWF preparations on platelet adhesion added to blood from VWD patients. Whole blood from two VWD patients (type 1 VWD and 2A VWD with $29 \%$ and $<10 \%$ VWF:RCo activity, respectively) was spiked with control VWF preparation or preparation $A$ or $B(2 \mathrm{IU} / \mathrm{mL})$, and then perfused over collagen type III at a shear a rate of $1700 \mathrm{~s}-1$. (A) Representative brightfield images after 4 min perfusion of spiked blood from type $2 \mathrm{~A}$ patient $(150 \times 150$ $\mu \mathrm{m})$. (B) Analyzed surface area coverage of platelets after perfusion of spiked blood from type 2A VWD patient (left side) and type 1 VWD patient (right side). Means \pm SEM ( $n=3$ independent experiments).

The literature provides indirect evidence that distinct triplet bands of the VWF multimers may differ in functional properties, but so far this has not directly been shown. For instance, an altered VWF triplet composition is detected in type $2 \mathrm{~A}$ or $2 \mathrm{~B}$ VWD, i.e. in forms of VWD that are characterized by altered VWF binding to platelets, likely as a consequence of changes in VWF proteolysis ${ }^{29,30}$. In other disease forms, like type 2M VWD, satellite bands can even be absent, despite the presence of normal or even supra-normal multimers, which associates with defects in platelet-VWF interaction 31.

For this paper, we have functionally characterized two VWF preparations separated by heparin affinity and size-exclusion chromatography, which are equal in VWF multimeric distribution patterns, but markedly differ in triplet band composition. Binding studies using SPR- and ELISA-based approaches indicate that preparation $\mathrm{A}$, lacking an extra $N$-terminal peptide sequence compared to preparation B, shows a significantly lower affinity for GPIb. In contrast, both preparations display an equal binding affinity for type III collagen. The relatively decreased function of preparation A is confirmed by two sets of flow studies, investigating platelet adhesion and thrombus formation due to interaction of the GPIb-V-IX complex with VWF. High-shear flow experiments over type III collagen, using either reconstituted blood ${ }^{21}$ or whole blood from VWD patients ${ }^{22}$, thus show significantly lower platelet deposition and thrombus formation in the presence of preparation A than of preparation B. Together, these finding suggest that the larger size VWF triplet bands, containing an additional $\mathrm{N}$-terminal peptide sequence, are more potent in recruiting platelets under arterial flow conditions. However, a limitation of our work is that the heparin fractionation had resulted in the partial loss of very high VWF multimers.

The presence or absence of the $\mathrm{N}$-terminal fragment with GPIb binding site in the A1 domain may be considered theoretically negligible for platelet recruitment, taking into account the overall sum of other GPIb binding sites present in VWF multimers. However, given the present results, it is well feasible that this one additional GPIb binding site, located at the outer surface of globular 
multimers, has a considerable impact on protein function, with regard to its sensitivity to conformational transition in response to fluid dynamic conditions and mechanical forces. Others have also reported on functional consequences due to the absence of the heparin-binding site within the A1 domain of VWF, following ADAMTS-13 cleavage ${ }^{32,33}$. The present results suggest that different functional properties of individual triplet bands are not only relevant for heparin affinity, but also for GPIb binding affinity.

Overall, the present results point to a distinct role of the VWF triplet band composition regarding GPIb-dependent platelet adhesion to VWF. The larger size, slower migrating VWF triplet bands appear to be functionally more active in supporting thrombus formation in collagen type III surfaces. The implication of these findings is that the altered triplet band composition, regularly observed in type 2 VWD patients, may contribute to an altered VWF-dependent platelet adhesion at high-shear flow.

\section{Acknowledgments}

We acknowledge support from the Cardiovascular Centre (HVC) Maastricht and the Center for Translational Molecular Medicine (CTMM) INCOAG, The Netherlands. We appreciate analytical support from A. Hillarp, (Malmö University Hospital, Sweden) and thank Dr. G. Kohla for critical review of the manuscript.

\section{References}

1. Ruggeri ZM. Platelet and von Willebrand factor interactions at the vessel wall. Hämostaseologie 24, 1-11 (2004).

2. Savage B, Saldivar E, Ruggeri ZM. Initiation of platelet adhesion by arrest onto fibrinogen or translocation on von Willebrand factor. Cell 84, 289-97(1996).

3. Hoylaerts MF, Yamamoto H, Nuyts K, Vreys I, Deckmyn H, Vermylen J. von Willebrand factor binds to native collagen VI primarily via its A1 domain. Biochem J 324, (Pt 1) 185-91 (1997).

4. Pareti FI, Niiya K, McPherson JM, Ruggeri ZM. Isolation and characterization of two domains of human von Willebrand factor that interact with fibrillar collagen types I and III. J Biol Chem 262, 13835-41 (1987).

5. Roth GJ, Titani K, Hoyer LW, Hickey MJ. Localization of binding sites within human von Willebrand factor for monomeric type III collagen. Biochemistry 25, 8357-61 (1986).

6. Cosemans JM, Schols SE, Stefanini L et al. Key role of glycoprotein Ib/V/IX and von Willebrand factor in platelet activation-dependent fibrin formation at low shear flow. Blood 117, 651-60 (2011).

7. Matsushita T, Dong Z, Sadler JE. Von Willebrand's factor and von Willebrand's disease. Curr Opin Hematol 1, 362-8 (1994)

8. Dong JF. Cleavage of ultra-large von Willebrand factor by ADAMTS-13 under flow conditions. $J$ Thromb $\begin{array}{llll}\text { Haemost } & \mathbf{3}, 1710-6 & \text { (2005). }\end{array}$

9. Dent JA, Galbusera M, Ruggeri ZM. Heterogeneity of plasma von Willebrand factor multimers resulting from proteolysis of the constituent subunit. J Clin Invest 88, 774-82 (1991)

10. Furlan $M$, Robles $R$, Affolter D, Meyer $D$, Baillod $P$, Lammle B. Triplet structure of von Willebrand factor reflects proteolytic degradation of high molecular weight multimers. Proc Natl Acad Sci USA 90, 7503-7 (1993).

11. Fischer $\mathrm{BE}$, Thomas $\mathrm{KB}$, Schlokat $\mathrm{U}$, Dorner F. Triplet structure of human von Willebrand factor. Biochem J 331, (Pt 2) 483-8 (1998).

12. Fischer BE, Thomas KB, Schlokat U, Dorner F. Selectivity of von Willebrand factor triplet bands towards heparin binding supports structural model. Eur J Haematol 62, 169-73 (1999).

13. Keuren JF, Ulrichts $\mathrm{H}$, Feijge MA et al. Integrin $\mathrm{a}_{\mathrm{IIb}} \beta_{3}$ and shear-dependent action of glycoprotein Ibalpha stimulate platelet-dependent thrombin formation in stirred plasma. J Lab Clin Med 141, 350-8 (2003)

14. Neugebauer BM, Goy C, Budek I, Seitz R. Comparison of two von Willebrand factor collagen-binding assays with different binding affinities for low, medium, and high multimers of von Willebrand factor. Semin Thromb Hemost 28, 139-48 (2002).

15. Budde U, Schneppenheim R, Plendl H, Dent J, Ruggeri ZM, Zimmerman TS. Luminographic detection of von Willebrand factor multimers in agarose gels and on nitrocellulose membranes. Thromb Haemost 63, 312-5 (1990). 
16. Matsubara Y, Murata M, Sugita K, Ikeda Y. Identification of a novel point mutation in platelet glycoprotein Iba, Gly to Ser at residue 233, in a Japanese family with platelet-type von Willebrand disease. J Thromb Haemost 1, 2198-205 (2003).

17. Russell SD, Roth GJ. Pseudo-von Willebrand disease: a mutation in the platelet glycoprotein Iba gene associated with a hyperactive surface receptor. Blood 81,1787-91 (1993).

18. Schneppenheim R, Obser T, Budde U, Patzke J. Development of a new functional assay for von Willebrand factor binding to platelet GpIba that does not require Ristocetin. Hämostaseologie 30, A28 (2010).

19. Li F, Moake JL, McIntire LV. Characterization of von Willebrand factor interaction with collagens in real time using surface plasmon resonance. Ann Biomed Eng 30, 1107-16 (2002).

20. Romijn RA, Westein $E$, Bouma $B$ et al. Mapping the collagen-binding site in the von Willebrand factor-A3 domain. J Biol Chem 278, 15035-9 (2003).

21. Fuchs B, Budde U, Schulz A, Kessler CM, Fisseau C, Kannicht C. Flow-based measurements of von Willebrand factor (VWF) function: binding to collagen and platelet adhesion under physiological shear rate. Thromb Res 125, 239-45 (2010).

22. Kuijpers $M J$, Schulte $V$, Bergmeier $W$ et al. Complementary roles of glycoprotein VI and $a_{2} \beta_{1}$ integrin in collagen-induced thrombus formation in flowing whole blood ex vivo. FASEB J 17, 685-7 (2003).

23. Munnix IC, Kuijpers MJ, Auger J et al. Segregation of platelet aggregatory and procoagulant microdomains in thrombus formation: regulation by transient integrin activation. Arterioscler Thromb Vasc Biol 27, 2484-90 (2007).

24. Munnix IC, Gilio K, Siljander P, Raynal N, Feijge MA, Hackeng TM, Deckmyn H, Smethurst PA, Farndale RW, Heemskerk JW. Collagen-mimetic peptides mediate flow-dependent thrombus formation by high- or low-affinity binding of integrin $a_{2} \beta_{1}$ and glycoprotein VI. J Thromb Haemost 6, 2132-42 (2008).

25. De Meyer SF, Vanhoorelbeke K, Ulrichts H, Staelens S, Feys HB, Salles I, Fontayne A, Deckmyn $\mathrm{H}$. Development of monoclonal antibodies that inhibit platelet adhesion or aggregation as potential anti-thrombotic drugs. Cardiovasc Hematol Disord Drug Targets 6, 191-207 (2006).

26. Budde U, Pieconka A, Will K, Schneppenheim R. Laboratory testing for von Willebrand disease: contribution of multimer analysis to diagnosis and classification. Semin Thromb Hemost 32, 514-21 (2006).

27. Furlan $M$, Robles $R$, Lammle B. Partial purification and characterization of a protease from human plasma cleaving von Willebrand factor to fragments produced by in vivo proteolysis. Blood 87, 4223-34 (1996).

28. Ruggeri ZM. Von Willebrand factor: looking back and looking forward. Thromb Haemost 98, 55-62 (2007).

29. Michiels JJ, Gadisseur A, Budde U, Berneman Z, Van der Planken M, Schroyen W, Van de Velde A, Van Vliet H. Characterization, classification, and treatment of von Willebrand diseases: a critical appraisal of the literature and personal experiences. Semin Thromb Hemost 31, 577-601 (2005).

30. Michiels J], Berneman Z, Gadisseur A, Van der Planken M, Schroyen W, Van de Velde A, Van Vliet $\mathrm{H}$. Classification and characterization of hereditary types $2 \mathrm{~A}, 2 \mathrm{~B}, 2 \mathrm{C}, 2 \mathrm{D}, 2 \mathrm{E}, 2 \mathrm{M}, 2 \mathrm{~N}$, and $2 \mathrm{U}$ (unclassifiable) von Willebrand disease. Clin Appl Thromb Hemost; 12, $397-420$ (2006).

31. Studt JD, Budde U, Schneppenheim R, Eisert R, von Depka Prondzinski M, Ganser A, Barthels M. Quantification and facilitated comparison of von Willebrand factor multimer patterns by densitometry. Am J Clin Pathol 116, 567-74 (2001).

32. Mohri H, Fujimura Y, Shima M, Yoshioka A, Houghten RA, Ruggeri ZM, Zimmerman TS. Structure of the von Willebrand factor domain interacting with glycoprotein Ib. J Biol Chem 263, 17901-4 (1988).

33. Sobel M, Soler DF, Kermode JC, Harris RB. Localization and characterization of a heparin binding domain peptide of human von Willebrand factor. J Biol Chem 267, 8857-62 (1992). 



\section{CHAPTER 6}

SUPPORTING ROLES OF PLATELET

THROMBOSPONDIN-1 AND CD36

IN THROMBUS FORMATION ON

COLLAGEN

Kuijpers MJ, de Witt SM, Nergiz-Unal R, van Kruchten R, Korporaal SJ, Verhamme P, Febbraio M, Tjwa M, Voshol PJ, Hoylaerts MF, Cosemans JM, Heemskerk JW 


\begin{abstract}
Platelets abundantly express the membrane receptor CD36 and store its ligand thrombospondin-1 (TSP1) in the a-granules. We investigated whether released TSP1 can support platelet adhesion and thrombus formation via interaction with CD36. Mouse platelets deficient in CD36 showed reduced adhesion to TSP1 and subsequent phosphatidylserine expression. Deficiency in either CD36 or TSP1 resulted in markedly increased dissolution of thrombi formed on collagen, although thrombus build-up was unchanged. In mesenteric vessels in vivo, deficiency in CD36 prolonged the time to occlusion and enhanced embolization, which was in agreement with earlier observations in TSP1-deficient mice. Thrombi formed using wildtype blood stained positively for secreted TSP1. Releasate from wildtype but not from TSP1-deficient platelets enhanced platelet activation, phosphatidylserine expression and thrombus formation on collagen. The enhancement was dependent on CD36, since it was without effect on thrombus formation by CD36-deficient platelets. These results demonstrate an anchoring role of platelet-released TSP1 via CD36 in platelet adhesion and collagen-dependent thrombus stabilization. Thus, the TSP1-CD36 tandem is another platelet ligand-receptor axis contributing to the maintenance of a stable thrombus.
\end{abstract}

\title{
Introduction
}

Glycoprotein IV or CD36 forms one of the most abundant glycoproteins on the surface of mouse and human platelets, expressed at up to 25,000 copies per cell ${ }^{1,2}$. CD36 consists of a double membrane-spanning protein with one large extracellular domain and two short $\mathrm{N}$ - and $\mathrm{C}$-terminal cytoplasmic domains ${ }^{3,4}$, 5 . Its function has remained unclear for long. Earlier, CD36 was believed to be a platelet collagen receptor 6,7 , but it was shown that platelets from CD36-deficient patients have an unchanged response to collagen ${ }^{8,9}$. Subsequent studies suggested a role for CD36 as receptor for thrombospondin-1 (TSP1) ${ }^{10}$, 11. It was also found that CD36 can bind oxidized lipids, including oxidized low density lipoproteins (oxLDL), particularly at conditions promoting atherogenesis ${ }^{12,13}$. Earlier, we and other have demonstrated that interaction of CD36 with surface-immobilized TSP1 or oxLDL leads to outside-in signaling events in platelets via the protein kinases JNK and Syk, and enforced via autocrine loops of secreted ADP and P2Y ${ }_{12}$ receptors ${ }^{14,15}$. Interestingly, this signalling promotes $\mathrm{Ca}^{2+}$-dependent exposure of procoagulant phosphatidylserine at the platelet surface.

The multi-domain matrix glycoprotein TSP1 is one of the most highly expressed proteins in platelet a-granules ( $\sim 101,000$ copies per cell) $)^{2}$. TSP1 is also found in the blood vessel, likely after deposition secreted by platelets and vascular cells ${ }^{16,17,18}$. In addition to CD36, several other platelet receptors for TSP1 have been proposed. These include the glycoprotein Ib-V-IX complex ${ }^{19,20,21}$. and glycoprotein CD4722. TSP1 can also indirectly influence platelet activity via binding to collagen, fibrinogen and von Willebrand factor (VWF), and protect the latter from cleavage by matrix proteinases ${ }^{23}$.

Lack of platelet a-granules is associated with a mild to moderate bleeding tendency in patients with the congenital gray platelet syndrome ${ }^{24}$. The genetic defect has recently been attributed to mutations in the neurobeaching-like 2 (NBEAL2) gene ${ }^{25,26}$. In mice deficiency in Nbeal2 similarly results in defective a-granule biogenesis, accompanied by diminished platelet adhesion, phosphatidylserine exposure and thrombus formation ${ }^{27}$. So far, it has remained unclear which of the proteins stored in platelet a-granules contribute to these responses.

In the present paper, we hypothesized that the abundantly expressed TSP1, secreted from the a-granules and interacting with its counter-receptor CD36, starts an additional autocrine feed-forward loop and supports the interactions of platelets in a thrombus. This hypothesis was tested using mice deficient in either CD36 or TSP1 by assessment of platelet adhesion, activation and thrombus formation. 


\section{Materials and Methods}

\section{Materials}

$\mathrm{H}$-Phe-Pro-Arg chloromethyl ketone (PPACK) and TSP1 from human platelets were obtained from Calbiochem (La Jolla CA, USA). Bovine serum albumin (BSA), ADP and thrombin and $\mathrm{FeCl}_{3}$ were from Sigma-Aldrich (St. Luis MO, USA). Low molecular weight heparin (fragmin) was from Pfizer (Capelle a/d IJssel, The Netherlands). Glycoprotein VI agonist, convulxin, was purified to homogeneity from the crude venom of Crotalus durissus terrificus (Latoxan, Valence, France) ${ }^{28}$. Fibrillar Horm type I collagen was from Nycomed Pharma (Munich, Germany). Annexin A5 (detecting surface-exposed phosphatidylserine) labeled with fluorescein isothiocyanate (FITC) was from PharmaTarget (Maastricht, the Netherlands). FITC-labeled anti-mouse CD62P mAb (detecting surface exposure of granular P-selectin) and PE-labeled JON/A mAb (detecting the activated conformation of mouse integrin $\mathrm{a}_{\mathrm{IIb}} \beta_{3}$ ) were from Emfret Analytics (Würzburg, Germany); biotin-conjugated anti-mouse TSP1 mAb from Abcam (Cambridge, UK); blocking anti-mouse CD36 mAb (MAB1258, clone 63) from Millipore (Temecula, CA, USA); blocking antibody against mouse CD47 (clone miap301) from eBiosciences (San Diego, CA, USA). Annexin A5 labeled with Alexa fluor (AF)647 and streptavidin labeled with AF532 were from Molecular Probes (Eugene OR, USA). Rhodamine 6G was from Invitrogen (Grand Island, NY, USA). Other materials were from sources described before ${ }^{29}$.

\section{Animals}

Experiments were approved by the local Animal Experimental Committees. Mice homozygous in CD36 deficiency $\left(\mathrm{Cd}^{2} 6^{-/}\right)$were generated as described, and crossed back $>6$ times to a C57BI/6 genetic background ${ }^{30}$. Mice deficient in TSP1 (Tsp1/-) also of C57BI/6 background were kindly provided by dr. J. Lawler (Harvard Medical School, Boston MA, USA) ${ }^{31}$. Wild type mice (C57BI/6) were used from the same sources. Blood counts of platelets and erythrocytes of knockout and wild type mice were in the normal ranges.

\section{Blood collection and platelet preparation}

Mice were anesthetized by subcutaneous injection of ketamine and xylazine $(0.1 \mathrm{mg} / \mathrm{g}$ and 0.02 $\mathrm{mg} / \mathrm{g}$ body weight), after which blood was obtained by retro-orbital puncture. For flow studies, blood was collected into $40 \mu \mathrm{mol} / \mathrm{L}$ PPACK, $5 \mathrm{U} / \mathrm{mL}$ heparin and $40 \mathrm{U} / \mathrm{mL}$ fragmin. For platelet preparation, blood was collected into acid citrate dextrose (ACD). Isolated platelets were washed and resuspended in modified Tyrode's Hepes buffer $(5 \mathrm{mmol} / \mathrm{L}$ Hepes, $136 \mathrm{mmol} / \mathrm{L} \mathrm{NaCl}, 2.7$ $\mathrm{mmol} / \mathrm{L} \mathrm{KCl}, 0.42 \mathrm{mmol} / \mathrm{L} \mathrm{NaH} \mathrm{PO}_{4}, 2 \mathrm{mmol} / \mathrm{L} \mathrm{MgCl}{ }_{2}, 0.1 \%$ glucose and $\left.0.1 \% \mathrm{BSA}, \mathrm{pH} 7.45\right)^{29}$. Platelet count was determined with a Coulter counter.

To prepare platelet releasates, aliquots of suspended Tsp1+/+ or Tsp1/- platelets $\left(1 \times 10^{9} /\right.$ $\mathrm{mL})$ were activated with thrombin $(20 \mathrm{nmol} / \mathrm{L})$ for 10 minutes, after which PPACK $(200 \mathrm{nmol} / \mathrm{L})$ was added to inactivate residual thrombin. Releasates were isolated by centrifuging for 1 minute at $2650 \mathrm{~g}$, and were stored at $-80^{\circ} \mathrm{C}$ until use.

\section{Flow cytometry}

Washed platelets $\left(1 \times 10^{8} / \mathrm{mL}\right)$ in modified Tyrode's buffer with $2 \mathrm{mmol} / \mathrm{L} \mathrm{CaCl}$ were activated with ADP $(0.5-5 \mu \mathrm{mol} / \mathrm{L})$ or convulxin $(20-50 \mathrm{ng} / \mathrm{mL})$ without stirring. After 10 minutes, activation of integrin $\mathrm{a}_{\mathrm{IIb}} \beta_{3}$ a-granule secretion, and phosphatidylserine exposure were detected with PE-labeled JON/A mAb (150 $\mathrm{mg} / \mathrm{mL})$, FITC-labeled anti-CD62P mAb (1:40) and AF647-labeled annexin A5 $(1 \mu \mathrm{g} / \mathrm{mL})$, respectively ${ }^{32}$. Fluorescence was measured with a BD Accuri C6 flow cytometer (San Jose, CA, USA).

\section{Static platelet adhesion and activation}

Round glass coverslips were coated with purified TSP1 $(3 \mu \mathrm{L}, 100 \mu \mathrm{g} / \mathrm{mL})$ or releasate from acti- 
vated platelets ( $3 \mu \mathrm{L}, \sim 3 \times 10^{6}$ platelets), and blocked with 1\% BSA in modified Tyrode's buffer. Coverslips were mounted in an open chamber and incubated with platelets in buffer containing 2 $\mathrm{mM} \mathrm{CaCl}_{2}\left(1.5 \times 10^{8} / \mathrm{mL}\right)$. Platelet adhesion was determined by phase-contrast microscopy, and exposure of phosphatidylserine by labeling with FITC-annexin A5 and fluorescence microscopy ${ }^{33}$.

\section{Thrombus formation under flow}

Rectangular coverslips were coated with fibrillar type I collagen ( $3 \mu \mathrm{L}, 50 \mu \mathrm{g} / \mathrm{mL})$, and blocked with $1 \%$ BSA in modified Tyrode's buffer. Where indicated, coated coverslips were post-incubated with releasate from activated platelets ( $3 \mu \mathrm{L}, \sim 3 \times 10^{6}$ platelets). After mounting into a transparent parallel-plate flow chamber (depth $50 \mu \mathrm{m}$, width $3 \mathrm{~mm}$ ), coverslips were perfused with PPACK/ heparin-anticoagulated mouse blood for 4 minutes at a wall shear rate of $1000 \mathrm{~s}^{-1}$ (Ref. 29) Using confocal microscopy, surface expression of activation markers on adhered platelets was measured by staining with PE-labeled JON/A mAb (probing activated integrin $\mathrm{a}_{\mathrm{IIb}} \beta_{3}$ ) and AF647-annexin A5 (probing phosphatidylserine exposure) ${ }^{32}$. Using Metamorph software (Molecular Devices, Sunnyvale CA, USA), brightfield and fluorescence images were analyzed for surface area coverage, mean thrombus size, or fluorescence intensity ${ }^{34}$. Where indicated, images were recorded with an EVOS inverted digital fluorescence microscope (AMG, Bothell WA, USA).

Thrombus stability was assessed by post-perfusion of thrombi with modified Tyrode's buffer containing $1 \mathrm{U} / \mathrm{mL}$ heparin for 6 minutes, during which period images were taken every 30 seconds $^{38}$. Differential images were obtained with Metamorph software, and analyzed for changes in pixel intensity above background.

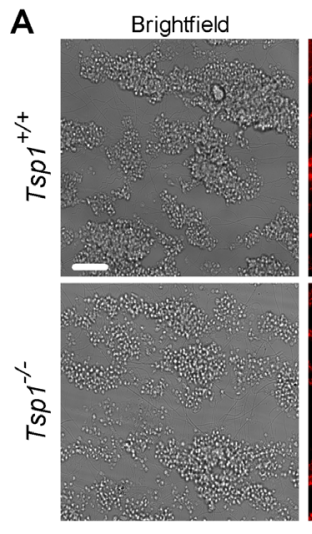

B

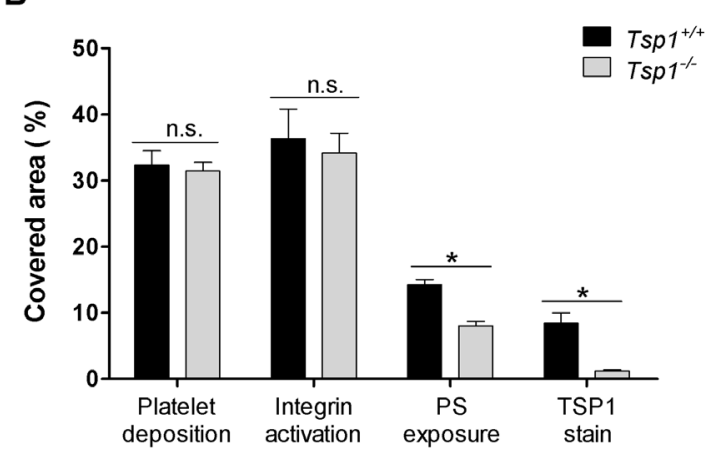

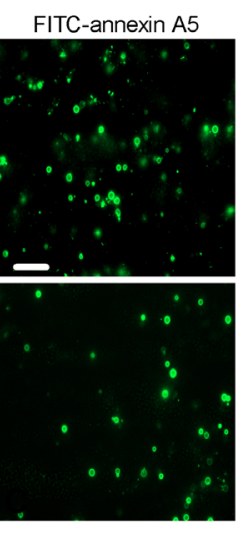

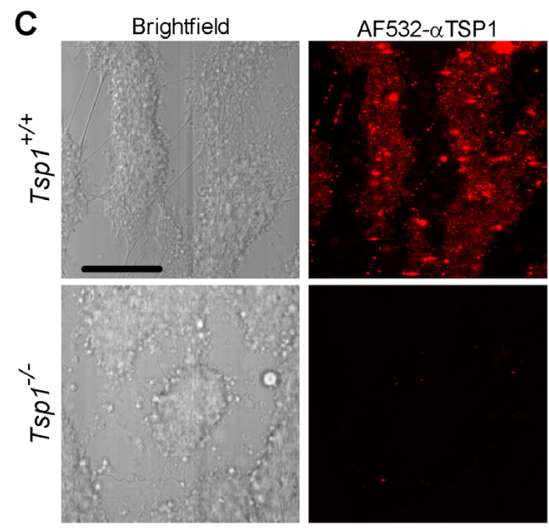

D

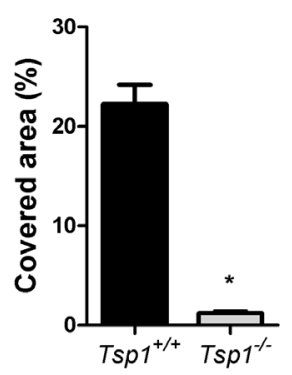

Figure 1. TSP1 released from platelets during thrombus formation supports phosphatidylserine exposure. (A-B) Blood from Tsp1 $1^{++}$or Tsp1/- mice was perfused during 4 minutes over collagen at a wall shear rate of $1000 \mathrm{~s}^{-1}$. (A) Representative brightfield and fluorescence images recorded after post-staining (bars, $25 \mu \mathrm{m}$ ). (B) Quantification of covered area of all platelets (platelet deposition); labelling with PE-JON/A mAb ( $\mathrm{a}_{\mathrm{IIb}} \beta_{3}$ activation); labelling with AF647-annexin A5 (PS exposure); and staining with biotin anti-TSP1 mAb followed by AF532-streptavidin (TSP1 stain). (C-D) $T s p 1^{+/+}$or $T s p 1^{-/-}$thrombi formed on collagen in the presence of ADP $(10 \mu \mathrm{mol} / \mathrm{L})$, staining with biotin anti-TSP1 mAb and AF532-streptavidin. (C) Representative images (bars, $25 \mu \mathrm{m}$ ). (D) Quantification of covered area of TSP1stain. Means \pm SEM $(\mathrm{n}=3-4)$. $* P<0.05$ vs. Tsp1 ${ }^{+/+}$; n.s., not significant. 
A
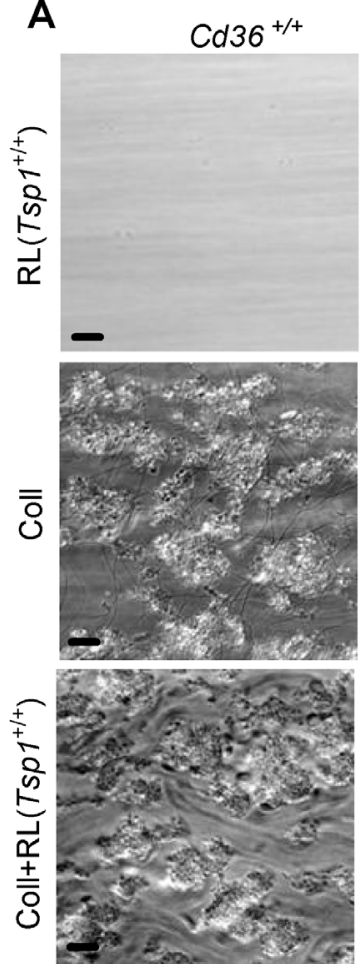

$\operatorname{cd} 36^{-1}$

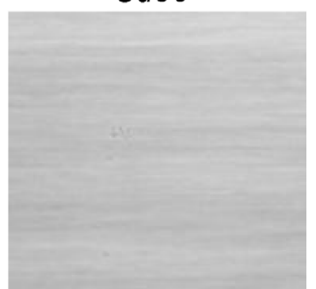

B

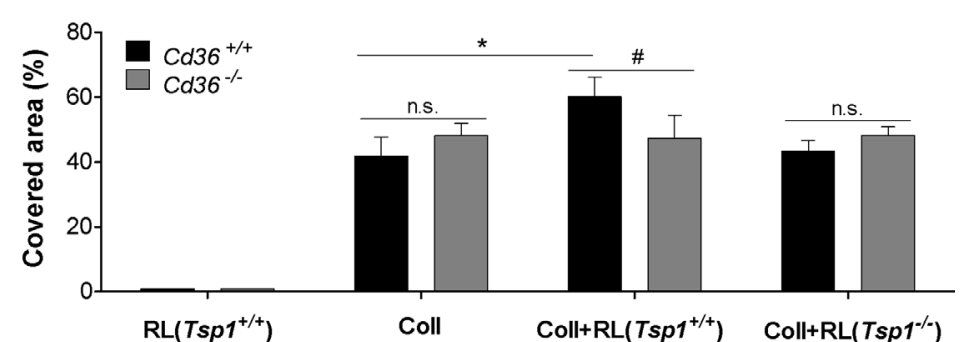

C

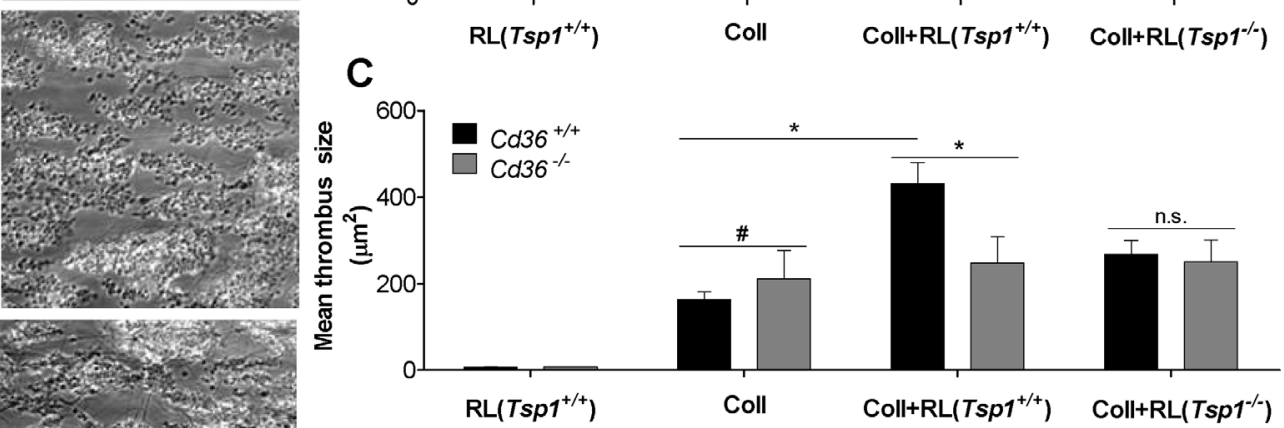

Figure 2. Platelet-derived TSP1 supports thrombus formation on collagen in a CD36-dependent way. Coverslips were coated with releasate (RL) from thrombin-stimulated Tsp $1^{+/+}$or Tsp $1^{--}$platelets, collagen alone (coll), or collagen post-incubated with RL, as indicated. Blood from $\mathrm{Cd} 36^{+/+}$or $C d 36^{-/-}$mice was perfused during 4 minutes at 1000 $\mathrm{s}^{-1}$. (A) Representative brightfield images of thrombi formed after flow (bars, $25 \mu \mathrm{m}$ ). (B) Quantification of platelet surface area coverage. (C) Morphometric analysis of images for mean thrombus size. Means $\pm \operatorname{SEM}(n=4-6) . * P<0.05$, ${ }^{\#} P<0.1$ vs. Cd36 ${ }^{+/+}$control.

\section{Immunostaining of thrombi}

Coverslips coated with collagen and/or platelet releasates, before or after thrombus formation, were stained with biotin-conjugated anti-murine TSP1 mAb $(4 \mu \mathrm{g} / \mathrm{mL})$ for 15 minutes. After rinse with modified Tyrode's buffer, the coverslips were post-stained with AF532-streptavidine (1:200) for 15 minutes. Control stainings were without primary mAb. No fixation or permeabilization was performed. Fluorescence images were recorded after a second rinse using an LSM7 Live confocal microscope (Zeiss, Jena, Germany).

\section{In vivo thrombosis in mesenteric vessels}

Mice were anesthetized by sodium pentobarbital (60 mg/ $\mathrm{kg}$, i.p.) and thrombus formation in the mesenteric vessels was provoked, as described previously ${ }^{35}$. Briefly, Rhodamine $6 \mathrm{G}$ was injected i.v. $(3.3 \mathrm{mg} / \mathrm{kg})$ for fluorescent labeling of circulating cells. After opening of the abdomen, mesenteric vessels were exposed on the table of an inverted epifluorescent microscope with 20x objective (Zeiss Observer, Jena, Germany), coupled to Axiovision software (Zeiss). A standardized vascular injury was induced by placement of a filter paper soaked with $5 \% \mathrm{FeCl}_{3}$ for 5 minutes. Platelet deposition, thrombus formation and embolization in arterioles and venules were monitored by microscopic fluorescence imaging in real-time until complete occlusion occurred. Time to occlusion was defined as the arrest of blood flow for at least 1 minute. Per animal, 2 measurements were performed. When occlusion had not occurred for 60 minutes, the experiment was terminated and occlusion time was determined as 60 minutes. 

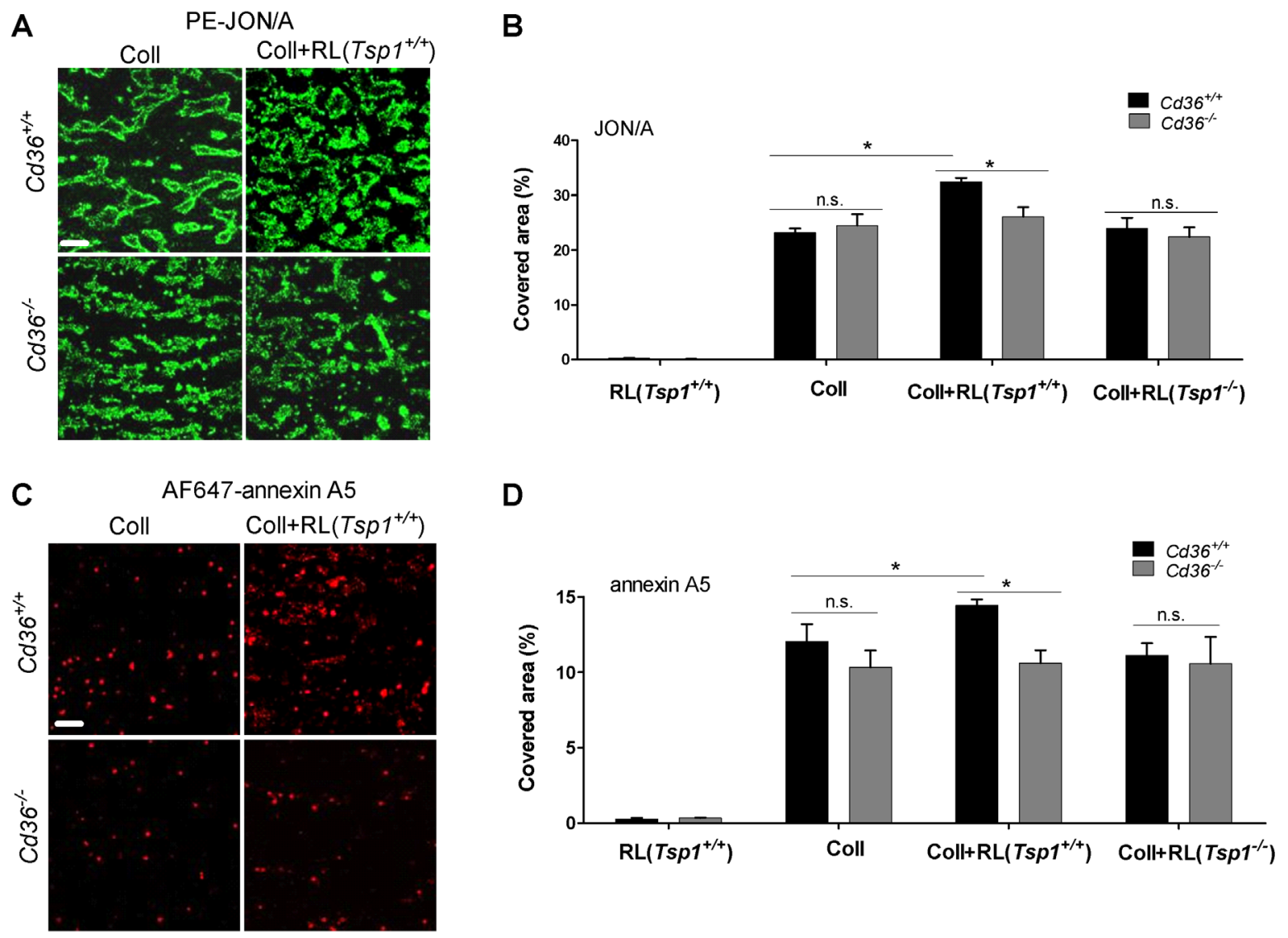

Figure 3. Platelet-derived TSP1 enhances phosphatidylserine exposure in thrombus formation in the presence of CD36. Blood from $\mathrm{Cd} 36^{+/+}$or $\mathrm{Cd} 36^{-/-}$mice was perfused during 4 minutes at $1000 \mathrm{~s}^{-1}$ over a surface of releasate (RL) from Tsp1+/+ platelets, over collagen alone (coll), or collagen post-incubated with RL from Tsp $1^{+/+}$or Tsp $1^{-/}$platelets, as indicated. (A,C) Representative fluorescence images (bars, $25 \mu \mathrm{m}$ ) of thrombi stained for activated $\mathrm{a}_{\mathrm{IIb}} \beta_{3}$ (PEJON/A mAb) and phosphatidylserine exposure (AF647-annexin A5). (B,D) Quantification of fluorescence area covered by thrombi. Means \pm SEM $(n=6-7) ; * P<0.05$ vs. Cd36 $6^{+/+}$control.

\section{Statistical analysis}

Significance of differences was determined with a parametric t-test or a non-parametric Mann-Whitney $\mathrm{U}$ test, as appropriate. The statistical package for social sciences was used (SPSS version 17, Chicago IL, USA).

\section{Results}

In human platelets, CD36 acts as an adhesive receptor for immobilized TSP1 ${ }^{15}$. To assess this for the mouse system, we measured the adhesion of platelets from $\mathrm{Cd} 36^{+/+}$and $\mathrm{Cd} 36^{-/}$mice to surfaces coated with purified human TSP1 or a TSP1-containing releasate from activated mouse platelets. With either surface, deficiency in CD36 resulted in a markedly reduced static adhesion (Suppl. Figure $1 \mathrm{~A}$ ). Labelling with FITC-annexin A5 indicated that the lower adhesion of Cd36platelets was accompanied by a lower phosphatidylserine exposure, suggesting reduced platelet activation (Suppl. Figure 1B). Immmunofluorescence experiments indicated that surface-coated releasate from wildtype platelets stained positively for TSP1, whereas surface-coated releasate from $T s p 1^{-}$platelets failed to stain (Suppl. Figure $2 A-B$ ). We determined whether phosphatidylserine-exposing microparticles as possible CD36 ligands ${ }^{36}$ may contribute to the adhesion of platelets to surface-coated releasates. However, staining of these coatings with FITC-annexin A5 did not result in a detectable fluorescence signal, pointing to the absence of microparticles on the coated 
surface (Suppl. Figure 3). Together, the data indicated that CD36 acts as an adhesive receptor for mouse platelets on released and immobilized TSP1.

To determine the activation tendency of platelets from mice, we measured integrin $a_{\text {IIb }} \beta_{3}$ activation, a-granule secretion and phosphatidylserine exposure in response to ADP or a glycoprotein VI agonist, convulxin. No difference was observed in any of the responses between the knockout and wildtype platelets (Suppl. Figure 1C-E). These results are in agreement with our previous data that blockage of CD36 does not affect agonist-induced activation of suspended human platelets. ${ }^{15}$ Similarly, other authors have described that CD36-deficient mouse platelets in suspension are unchanged in activation properties ${ }^{12,37}$.

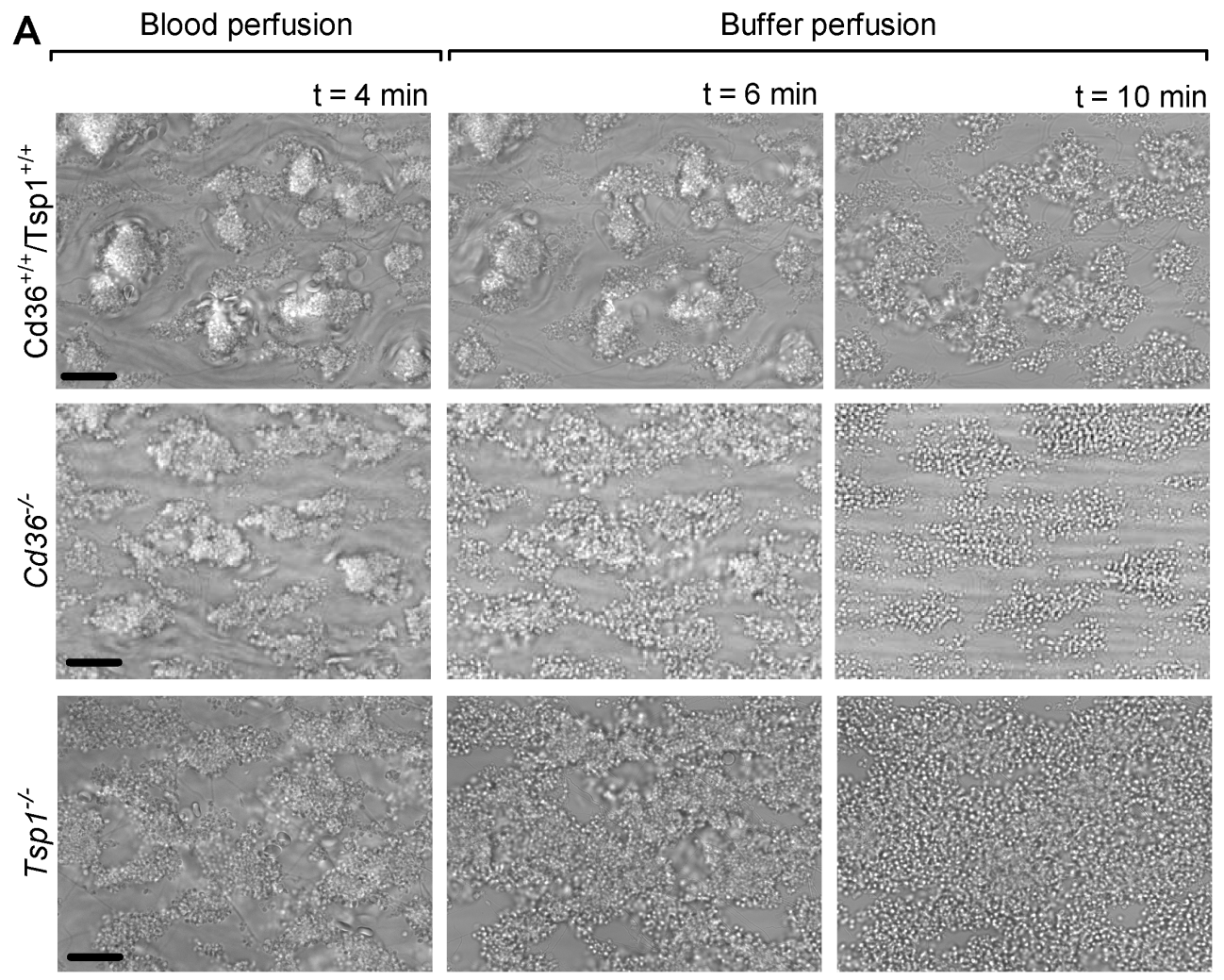

B

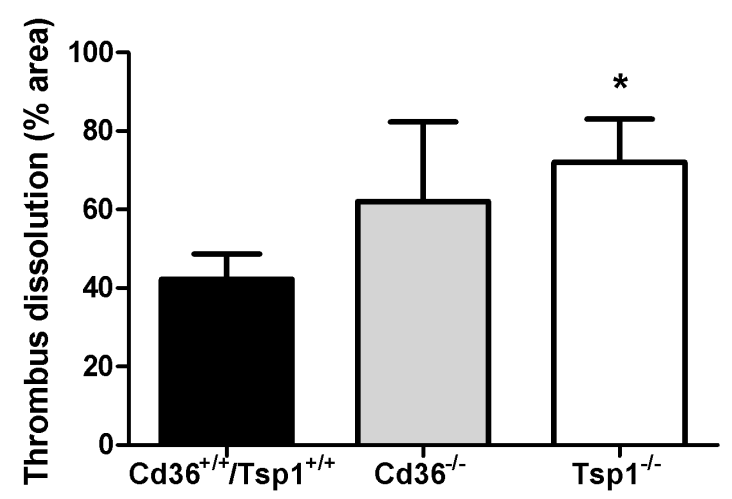

Figure 4. Platelet CD36 and TSP1 support thrombus stabilization on collagen. Thrombi were formed by 4 minutes perfusion over collagen at $1000 \mathrm{~s}^{-1}$, using blood from Cd36- $6^{-1}$, Tsp $1^{-1-}$ or corresponding wildtype mice. Stability of thrombi was evaluated during 6 minutes of post-perfusion with modified Tyrode's buffer, containing $2 \mathrm{mmol} / \mathrm{L} \mathrm{CaCl}$ and $1 \mathrm{U} / \mathrm{mL}$ heparin. (A) Representative phase-contrast images taken during post-perfusion (bars, $25 \mu \mathrm{m})$. (B) Dissolution of thrombi during post-perfusion calculated by subtraction analysis. Representative data of at least 4 experiments. See also movies in online-only supplement. 
A

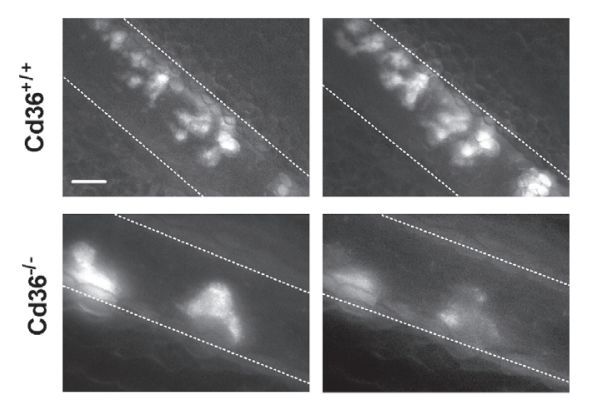

B
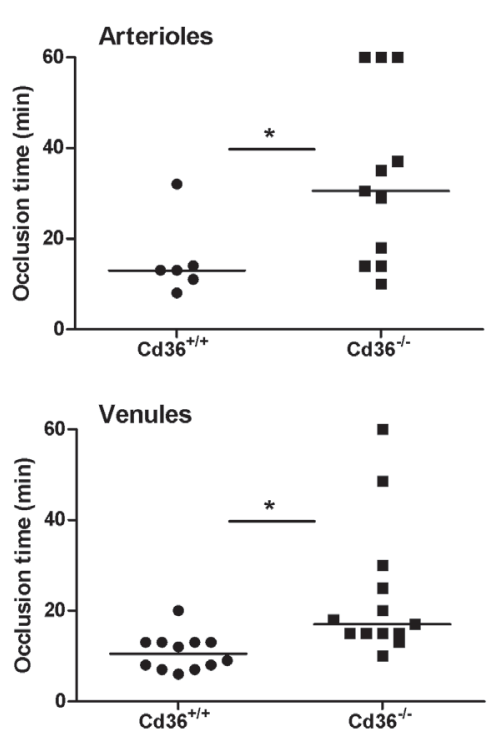

Figure 5. Platelet CD36 supports formation of stable thrombi in vivo. Thrombus formation was induced in mesenteric vessels from $\mathrm{Cd} 36^{+/+}$and $\mathrm{Cd} 36^{-/}$mice by $\mathrm{FeCl}_{3}$ injury. Animals were pre-injected with rhodamin $6 \mathrm{G}$ to fluorescently label circulating cells. (A) Representative images showing thrombus instability and embolization in injured arterioles from Cd36-- mice. Dotted lines indicate vessel walls; right image is 50 s later than left image (bar, $100 \mu \mathrm{m}$ ). See also movies in online-only supplement. (B) Distribution plots of occlusion times in arterioles (top panel) and venules (bottom panel) following injury. Horizontal lines represent median values $(* P<0.05)$.

Considering that CD36 mediates platelet adhesion to immobilized TSP1, we investigated whether platelet-released TSP1 contributes to thrombus formation under flow conditions. Whole blood from $T s p 1^{1}$ or corresponding wildtype mice was perfused over collagen at high wall shear rate. Thrombi formed with $T s p 1^{1-}$ and $T s p 1^{+/+}$blood differed neither in deposition of platelets (surface area coverage) nor in integrin $\mathrm{a}_{\mathrm{IIb}} \beta_{3}$ activation (staining with PE-JON/A mAb) (Figure 1A, B). However, thrombi formed with Tsp1/- blood were significantly reduced in platelet phosphatidylserine exposure. Immunostaining indicated the presence of released TSP1 in wildtype thrombi, but not in Tsp1/- thrombi. To stimulate activation and secretion, thrombi on collagen were treated with ADP, and then immunostained for TSP1 (Figure 1C, D). Strong staining of the Tsp $1^{+/+}$thrombi was observed, but not of the Tsp1\% thrombi.

The roles of CD36 and TSP1 in thrombus formation were studied using $\mathrm{Cd} 36^{-/}$-blood, which was perfused over collagen surfaces containing releasate from Tsp $1^{--}$or $T s p 1^{+/+}$platelets. Control experiments indicated that surfaces with only releasates did not result in adhesion of $\mathrm{Cd}_{36+/+}$ or Cd36 $6^{--}$platelets (Figure 2A, B). However, in combination with collagen, releasate from $\mathrm{Tsp}^{+/+}$ platelets but not from Tsp1\% platelets enhanced the process of thrombus formation in perfusions with $\mathrm{Cd} 36^{+/+}$blood only $(\mathrm{P}=0.035)$. Image analysis indicated that mean thrombus size was largest for the combination of Tsp $1^{+/+}$releasate and $C d 36^{+/+}$blood $(P=0.001$, Figure $2 \mathrm{C})$. When using $\mathrm{Cd} 36^{-/}$blood, no increase in thrombus size was observed in the presence of releasate from Tsp $1^{+/+}$ or Tsp1\% platelets.

Staining for integrin $\mathrm{a}_{\mathrm{IIb}} \beta_{3}$ activation did not show marked differences between $C d 36^{-/-}$and $C d 36^{+/+}$thrombi formed on collagen alone (Figure 3A, B). However, $\mathrm{a}_{\mathrm{IIb}} \beta_{3}$ activation and phosphatidylserine exposure were significantly increased in $C d 36^{+/+}$thrombi $(P=0.005$ and 0.027 , respectively), when these were formed on a surface with collagen plus releasate from $\mathrm{Tsp}^{+/+}$(but not $T s p 1^{-/}$) platelets (Figure 3A-D). Notably, the stimulating effect of $T s p 1^{+/+}$releasate was absent in Cd36- thrombi. Together, this pointed to a platelet-activating effect of immobilized TSP1 via CD36, thus supporting collagen-dependent thrombus formation and procoagulant activity. 
Considering that paracrine platelet agents like ADP, thromboxane, and Gas6 contribute to thrombus stabilization ${ }^{38,39}$, we investigated the stability of thrombi formed with $C d 36^{\%}$, Tsp $1^{-}$or wildtype blood. This was done by measuring the rate of dissolution of preformed thrombi during a high shear post-perfusion protocol ${ }^{38}$. Strikingly, whereas the wildtype thrombi remained stable for a longer period, the thrombi of platelets deficient in either CD36 or TSP1 disintegrated within several minutes (Figure 4A; see movies in the supplemental material). Quantification of thrombus dissolution during a 6 minutes period indicated a significant increase in this parameter for $\mathrm{Cd}_{3} \mathrm{6}^{-/}$ and $T s p 1^{-}$platelets in comparison to wildtype (Figure 4B).

Control experiments pointed out that in Tsp1\%- blood the blocking anti-mouse CD36 antibody MAB1258 did not have an additional effect (data not shown), suggesting that no other CD36 ligand was involved in thrombus stabilization. Furthermore, pretreatment of $\mathrm{Cd} 36^{+/+}$or $\mathrm{Cd} 36^{-\%}$ blood with a blocking antibody against CD47 did not change platelet deposition or thrombus stability (data not shown). Together, these findings pointed to a role of TSP1-CD36 interaction in the stabilization of thrombi at high shear flow.

To investigate this under in vivo conditions, we measured the thrombotic process in mesenteric arterioles and venules from $\mathrm{Cd}_{3} 6^{+/+}$and $\mathrm{Cd} 36^{-/}$mice, injured with $\mathrm{FeCl}_{3}$. In the vessels from $\mathrm{Cd} 36^{-/-}$mice, thrombus formation was delayed and more unstable, i.e. more embolization, in comparison to wildtype mice (Figure 5A, and movies in the online-only Supplement). This resulted in prolonged occlusion times in both the arterioles and venules of $\mathrm{Cd}_{36}{ }^{--}$mice (Figure 5B). These results are compatible with our earlier finding that also in mesenteric vessels of Tsp1\% mice thrombus formation is delayed and accompanied by embolization ${ }^{23}$. Together, these data indicate that both CD36 and TSP1 play a role in thrombus stabilization in vivo.

\section{Discussion}

In this study we hypothesized that platelet-secreted TSP1 by interacting with CD36 provides an additional autocrine feed-forward loop that enforces the interactions of platelets in a growing thrombus. The results of this paper agree with a stimulatory effect of the TSP1-CD36 axis in thrombus formation and stabilization. Our data indicate that, similarly to purified human TSP1, the releasate from wildtype but not from $T s p 1^{--}$platelets augments platelet adhesion and phosphatidylserine exposure in a CD36-dependent way, i.e. detectable with wildtype but not $\mathrm{Cd} 36^{-/}$platelets. In addition, we find that only the releasate from wildtype platelets enhances collagen-induced thrombus formation, integrinactivation and phosphatidylserine exposure at high shear flow conditions. Furthermore, we find that stable thrombus formation is impaired in mice deficient in TSP1 or CD36 both in in vitro and in vivo experiments. In vitro, we could establish that the impairment was not further increased by blockage of CD36.

Jointly, these results indicate that platelet-derived TSP1 and CD36 support adhesion and activation of platelets to PS exposure and thrombus stabilization. These are considered to be relevant findings since TSP1, although also present in the vessel wall, is accumulated within the blood in platelet a-granules ${ }^{2}, 16,17$. The TSP1 that is secreted from activated platelets thus may deposit on collagen or a growing thrombus, and then act in an autocrine way to enforce the thrombus-forming process. Interestingly, the present findings with $\mathrm{Cd}_{3} 6^{\%}$ and $\mathrm{Tsp} 1^{-/}$platelets are reminiscent - although with a less strong phenotype - to those published for $\mathrm{Nbeal} \%$ platelets. These platelets (lacking a-granules) show impaired adhesion, reduced phosphatidylserine exposure, and diminished thrombus formation accompanied by increased thrombus instability ${ }^{27}$. Hence, TSP1 may be one of the a-granular proteins contributing to full and stable thrombus formation. This idea is supported by the fact that both TSP1 and CD36 are highly expressed in (mouse and human) platelets, thus allowing multiple possible interaction sites. Likely, also other a-granule-derived proteins than TSP1 will play a role in thrombus formation, but we like to note that TSP1 - differently from VWF, fibrinogen and Gas6 - is mostly stored in the blood in platelets, and is present in plasma at only low concentrations. 
The present results are in agreement with earlier data, showing that immobilized TSP1 activates human platelets via CD36 through a Syk kinase-dependent mechanism, resulting in increased $\mathrm{Ca}^{2+}$ signaling, activation of $\mathrm{a}_{\mathrm{IIb}} \beta_{3}$, and exposure of phosphatidylserine ${ }^{15}$. Also in endothelial cells, a role for TSP1 in signaling via CD36 has been proposed ${ }^{40}$. Others have provided evidence that TSP1 can promote platelet activation via the glycoprotein CD4722. However, in our experiments, blocking CD47 did not influence the process of thrombus formation in the presence or absence of CD36. These experiments, however, do not rule out that TSP1 can act by binding to other platelet ligands in the extracellular space, e.g. VWF and fibrinogen. In the absence of TSP1 also other effects may occur, given the previously identified role of TSP1 in protecting VWF from ADAMTS-13 degradation $^{23}$. Similarly, in the absence of CD36, also the interaction with other ligands like oxLDL will be prevented. In other words, although the present results clearly point to an overlap of the functions of CD36 and TSP1 in thrombus formation, they do not impose that these functions are identical.

Various authors have pointed to a role of either CD36 or TSP1, although in a different context and not focusing on the TSP1-CD36 axis. Platelet CD36, by acting as a scavenging receptor of oxLDL, was found to contribute to atherosclerotic lesion development ${ }^{41}$, and hyperlipidemia-associated enhanced platelet reactivity ${ }^{12}$. The oxLDL-CD36 pathway is supposed to signal via the mitogen-activated protein kinases $\mathrm{JNK}^{14}$, and $\mathrm{p38MAPK}{ }^{13}$, and the tyrosine kinase Syk ${ }^{15}$.

In earlier work, we demonstrated that continued inside-out signaling via ADP/ADP receptors and Gas6/Gas6 receptors, as well as PEAR1, contributes to perpetuated $\mathrm{a}_{\mathrm{IIb}} \beta_{3}$ activation and maintenance of platelet-platelet interactions, and thereby to stabilization of a formed thrombus ${ }^{30,31,42}$. The present findings extend this concept by revealing the involvement of another autocrine axis in thrombus stabilization, namely the interaction of CD36 with platelet-derived TSP1.

Taken together, our results point to defined roles of murine CD36 and platelet-derived TSP1 in collagen-dependent thrombus formation under high shear flow conditions. Thus, TSP1 binding to platelet CD36 can be considered as another of the multiple receptor-ligand interactions required for the buildup of a stable thrombus.

\section{Acknowledgements}

We thank Dr. J. Lawler (Harvard Medical School, Boston MA, USA) and Dr. O. Blanc-Brude (Paris Center for Cardiovascular Research, INSERM) for making Tsp1 ${ }^{--}$mice available. We thank dr. I. Vroegrijk and T. Mastenbroek for technical assistance.

\section{Sources of Funding}

This work was supported by Hacettepe University (Ankara, Turkey) to R.N.U., the Netherlands Heart Foundation (2011T6) to J.M.E.M.C., the Center for Translational Molecular Medicine (INCOAG) and ZonMW-MKMD 114021004 to J.M.E.M.C. and J.W.M.H., and the Landsteiner Foundation for Blood Transfusion Research (0912F) to S.J.A.K.

\section{References}

1. Ghosh A, Murugesan G, Chen K, Zhang L, Wang Q, Febbraio M, Anselmo RM, Marchant K, Barnard J, Silverstein RL. Platelet CD36 surface expression levels affect functional responses to oxidized LDL and are associated with inheritance of specific genetic polymorphisms. Blood 117, 6355-6366 (2011).

2. Burkhart JM, Vaudel M, Gambaryan S, Radau S, Walter U, Martens L, Geiger J, Sickmann A, Zahedi RP. The first comprehensive and quantitative analysis of human platelet protein composition allows the comparative analysis of structural and functional pathways. Blood 120, e73-e82 (2012).

3. Silverstein RL, Febbraio M. CD36, a scavenger receptor involved in immunity, metabolism, angiogenesis, and behavior. Sci Signal 2, re3 (2009).

4. Glatz JF, Luiken JJ, Bonen A. Membrane fatty acid transporters as regulators of lipid metabolism: implications for metabolic disease. Physiol Rev 90, 367-417 (2010).

5. Nergiz-Unal R, Rademakers T, Cosemans JM, Heemskerk JW. CD36 as a multiple-ligand signaling receptor in atherothrombosis. Cardiovasc Hematol Agents Med Chem 9, 42-55 (2011).

6. Tandon NN, Kralisz U, Jamieson GA. Identification of glycoprotein IV (CD36) as a primary receptor for 
platelet-collagen adhesion. J Biol Chem 264, 7576-7583 (1989).

7. Diaz-Ricart M, Tandon NN, Carretero M, Ordinas A, Bastida E, Jamieson GA. Platelets lacking functional CD36 (glycoprotein IV) show reduced adhesion to collagen in flowing whole blood. Blood 82, 491496 (1993).

8. Daniel JL, Dangelmaier C, Strouse R, Smith JB. Collagen induces normal signal transduction in platelets deficient in CD36 (platelet glycoprotein IV). Thromb Haemost 71, 353-356 (1994).

9. Kehrel B, Wierwill S, Clemetson KJ, Anders O, Steiner M, Knight GC, Farndale RW, Okuma M, Barnes MJ. Glycoprotein VI is a major collagen receptor for platelet activation: it recognizes the platelet-activating quaternary structure of collagen, whereas CD36, glycoprotein IIb/IIIa, and von Willebrand factor do not. Blood 91, 491-499 (1998).

10. Leung LL, Li WX, McGregor JL, Albrecht G, Howard RJ. CD36 peptides enhance or inhibit CD36thrombospondin binding. A two-step process of ligand-receptor interaction. J Biol Chem 267, 1824418250 (1992).

11. Frieda S, Pearce A, Wu J, Silverstein RL. Recombinant GST/CD36 fusion proteins define a thrombospondin binding domain. Evidence for a single calcium-dependent binding site on CD36. J Biol Chem 270, 2981-2986 (1995).

12. Podrez EA, Byzova TV, Febbraio M, Salomon RG, Ma Y, Valiyaveettil M, Polikov E, Sun m, Finton PJ, Curtis BR, Chen J, Zhang R, Silverstein RL, Hazen SL. Platelet CD36 links hyperlipidemia, oxidant stress and a prothrombotic phenotype. Nat Med 13, 1086-1095 (2007).

13. Korporaal SJ, Van Eck M, Adelmeijer J, Ijseldijk M, Out R, Lisman T, Lenting PJ, Van Berkel TJ, Akkerman JW. Platelet activation by oxidized low density lipoprotein is mediated by CD36 and scavenger receptor-A. Arterioscler Thromb Vasc Biol 27, 2476-2483 (2007).

14. Chen K, Febbraio M, Li W, Silverstein RL. A specific CD36-dependent signaling pathway is required for platelet activation by oxidized low-density lipoprotein. Circ Res 102, 1512-1519 (2008).

15. Nergiz-Unal R, Lamers MM, Van Kruchten R, Luiken JJ, Cosemans JM, Glatz JF, Kuijpers MJ, Heemskerk JW. Signaling role of CD36 in platelet activation and thrombus formation on immobilized thrombospondin or oxidized low-density lipoprotein. J Thromb Haemost 9, 1835-1846 (2011).

16. Bornstein P. Thrombospondins as matricellular modulators of cell function. J Clin Invest 107, 929-934 (2001).

17. Bonnefoy A, Hantgan R, Legrand C, Frojmovic MM. A model of platelet aggregation involving multiple interactions of thrombospondin-1, fibrinogen, and GPIIbIIIa receptor. J Biol Chem 276, 5605-5612 (2001).

18. Tan K, Lawler J. The interaction of thrombospondins with extracellular matrix proteins. J Cell Commun Signal 3, 177-187 (2009).

19. Stomski FC, Gani JS, Bates RC, Burns GF. Adhesion to thrombospondin by human embryon fibroblasts is mediated by multiple receptors and includes a role for glycoprotein 88 (CD36). Exp Cell Res 198, 85-92 (1992).

20. Jurk K, Clemetson KJ, De Groot PG, Brodde MF, Steiner M, Savion N, Varon D, Sixma JJ, Van Aken H, Kehrel BE. Thrombospondin-1 mediates platelet adhesion at high shear via glycoprotein Ib (GPIb): an alternative/backup mechanism to von Willebrand factor. Faseb J 17, 1490-1492 (2003).

21. Isenberg JS, Wink DA, Roberts DD. Thrombospondin-1 antagonizes nitric oxide-stimulated vascular smooth muscle cell responses. Cardiovasc Res 71, 785-793 (2006).

22. Isenberg JS, Romeo MJ, Yu C, Yu CK, Nghiem K, Monsale J, Rick ME, Wink DA, Frzier WA, Roberts DD. Thrombospondin-1 stimulates platelet aggregation by blocking the antithrombotic activity of nitric oxide/cGMP signaling. Blood 111, 613-623 (2008).

23. Bonnefoy A, Daenens K, Feys HB, De Vos R, Vandervoort P, Vermylen J, Lawler J, Hoylaerts MF. Thrombospondin- 1 controls vascular platelet recruitment and thrombus adherence in mice by protecting (sub)endothelial VWF from cleavage by ADAMTS-13. Blood 107, 955-964 (2006).

24. Rosa JP, George JN, Bainton DF, Nurden AT, Caen JP, McEver RP. Gray platelet syndrome. Demonstration of alpha granule membranes that can fuse with the cell surface. J Clin Invest 80, 11381146 (1987).

25. Gunay-Aygun M, Falik-Zaccai TC, Vilboux T, Zivony-Elboum Y, Cetin M, Khayat M, Boerkoel CF, Kfir N, Huang Y, Maynard D, Dorward H, Berger K, Kleta R, Anikster Y, Arat M, Freiberg AS, Kehrel BE, Jurk $\mathrm{K}$, Cruz P, Mullikin JC, White JG, Huizing M, Gahl WA. NBEAL2 is mutated in gray platelet syndrome and is required for biogenesis of platelet alpha-granules. Nat Genet 43, $732-734$ (2011).

26. Albers CA, Cvejic A, Bouwmans EE, Alessi MC, Bertone P, Jordan P, Kettleborough RN, Kiddle G, Kostadima M, Read RJ, Sipos B, Sivapalaratnam S, Smethurst PA, Stephens J, Voss K, Nurden A, Rendon A, Nurden P, Ouwehand WH. Exome sequencing identifies NBEAL2 as the causative gene for 
gray platelet syndrome. Nat Genet 43, 735-737 (2011).

27. Deppermann C, Cherpokova, Nurden P, Schulz JN, Thielmann I, Kraft P, Vögtle T, Kleinschnitz C, Dütting S, Krohne G, Eming SA, Nurden AT, Eckes B, Stoll G, Stegner D, Nieswandt B. Gray platelet syndrome and defective thrombo-inflammation in Nbeal2-deficient mice. J Clin Invest, 123, 33313342 (2013).

28. Siljander P, Farndale RW, Feijge MA, Comfurius P, Kos S, Bevers EM, Heemskerk JW. Platelet adhesion enhances the glycoprotein VI-dependent procoagulant response: involvement of p38 MAP kinase and calpain. Arterioscler Thromb Vasc Biol. 21, 618-627 (2001).

29. Gilio K, Harper MT, Cosemans JM, Konopatskaya O, Munnix IC, Prinzen L, Leitges M, Liu Q, Molkentin JD, Heemskerk JW, Poole AW. Functional divergence of platelet protein kinase C (PKC) iso forms in thrombus formation on collagen. J Biol Chem 285, 23410-23419 (2010).

30. Febbraio M, Abumrad NA, Hajjar DP, Sharma K, Cheng W, Pearce SF, Silverstein RL. A null mutation in murine CD36 reveals an important role in fatty acid and lipoprotein metabolism. J Biol Chem 274, 19055-19062 (1999).

31. Lawler J, Sunday M, Thibert V, Duquette M, George EL, Rayburn H, Hynes RO. Thrombospondin-1 is required for normal murine pulmonary homeostasis and its absence causes pneumonia. $J$ Clin Invest 101, 982-992 (1998).

32. Munnix IC, Kuijpers MJ, Auger JM, Thomassen CM, Panizzi P, van Zandvoort MA, Rosing J, Bock PE, Watson SP, Heemskerk JW. Segregation of platelet aggregatory and procoagulant microdomains in thrombus formation. Regulation by transient integrin activation. Arterioscler Thromb Vasc Biol 27, 2484-2490 (2007).

33. Siljander PR, Munnix IC, Smethurst P, Deckmyn H, Lindhout T, Ouwehand WH, Farndale RW, Heemskerk JW. Platelet receptor interplay regulates collagen-induced thrombus formation in flowing human blood. Blood 103, 1333-1341 (2004).

34. Van Kruchten R, Cosemans JMEM, Heemskerk JWM. Measurement of whole blood thrombus formation using parallel-plate flow chambers: a practical guide. Platelets 23, 229-242 (2012).

35. Emmerechts J, Vanassche T, Loyen S, Van Linthout I, Cludts K, Kauskot A, Long C, Jacquemin M, Hoylaerts MF, Verhamme P. Partial versus complete factor VIII inhibition in a mouse model of venous thrombosis. Thromb Res 129, 514-519 (2012).

36. Ghosh A, Li W, Febbraio M, Espinola RG, McCrae KR, Cockrell E, Silverstein RL. Platelet CD36 mediates interactions with endothelial cell-derived microparticles and contributes to thrombosis in mice. J Clin Invest 118, 1934-1943 (2008).

37. Brodde MF, Korporaal SJ, Herminghaus G, Fobker M, Van Berkel TJ, Tietge UJ, Robenek H, Van Eck M, Kehrel BE, Nofer JR. Native high-density lipoproteins inhibit platelet activation via scavenger receptor BI: role of negatively charged phospholipids. Atherosclerosis 215, 374-382 (2011).

38. Cosemans JM, Munnix IC, Wetzker R, Heller R, Jackson SP, Heemskerk JW. Continuous signaling via PI3K isoforms beta and gamma is required for platelet ADP receptor function in dynamic thrombus stabilization. Blood 108, 3045-3052 (2006).

39. Cosemans JM, Van Kruchten R, Olieslager S, Schurgers LJ, Verheyen FK, Munnix IC, Waltenberger J, Angelillo-Scherrer A, Hoylaerts MF, Carmeliet P, Heemskerk JW. Potentiating role of Gas6 and Tyro3, Axl and Mer (TAM) receptors in human and murine platelet activation and thrombus stabilization. $J$ Thromb Haemost 8, 1797-1808 (2010).

40. Chu LY, Ramakrishnan DP, Silverstein RL. Thrombospondin-1 modulates VEGF signaling via CD36 by recruiting SHP-1 to VEGFR2 complex in microvascular endothelial cells. Blood 122, 1822-1832 (2013).

41. Febbraio M, Podrez EA, Smith JD, Hajjar DP, Hazen SL, Hoff HF, Sharma K, Silverstein RL. Targeted disruption of the class $\mathrm{B}$ scavenger receptor CD36 protects against atherosclerotic lesion development in mice. J Clin Invest 105, 1049-1056 (2000).

42. Kauskot A, Di Michele M, Loyen S, Freson K, Verhamme P, Hoylaerts MF. A novel mechanism of sustained platelet $\mathrm{a}_{\mathrm{III}} \beta_{3}$ activation via PEAR1. Blood 119, 4056-4065 (2012). 


\section{CHAPTER 7}

\section{INSIGHTS INTO PLATELET-BASED CONTROL OF COAGULATION}

De Witt SM, Verdoold R, Cosemans JM, Heemskerk JW 


\begin{abstract}
The coagulation process is activated by tight control mechanisms, in which platelets play prominent and unique roles. In thrombosis and hemostasis, activated platelets control the coagulation system in various ways: by exposing a phosphatidylserine surface for thrombin formation, by supporting fibrin formation, and by regulating the retraction of a fibrin clot. In this review we discuss the involvement of platelet receptors, other membrane proteins, downstream signaling proteins, cytoskeleton-linked proteins and plasma proteins in these coagulant functions. Studies with both genetically modified mice and pharmacological inhibitors indicate that, in collagen-adhered platelets, in part common signaling pathways lead to phosphatidylserine exposure, generation of thrombin and fibrin, and retraction of the fibrin clot. However, prolonged $\mathrm{Ca}^{2+}$ elevation leads to thrombin generation, whereas integrin-dependent signaling stimulates fibrin clot retraction. Contact-dependent signaling pathways, triggered by homotypic platelet-platelet interactions, seem to act in particular via the integrin route.
\end{abstract}

\title{
Introduction
}

The coagulation process is regulated by tight control mechanisms. During thrombosis and hemostasis, platelets provide prominent and unique roles in supporting and confining the coagulant activity ${ }^{1,2}$. Particularly studies with genetically modified mice and with inhibitors using human platelets have provided detailed insights into the molecular determinants of platelet-based coagulation. In this review, we discuss these findings regarding three mechanisms, how platelets: (i) form a procoagulant surface with exposed phosphatidylserine; (ii) provide sites for thrombin and fibrin formation; and (iii) steer fibrin clot retraction (Figure 1).

\section{Regulation of platelet phosphatidylserine exposure}

Flow chamber devices have extensively been used to study the process of thrombus formation at arterial shear conditions in order to evaluate not only platelet aggregation, but also platelet procoagulant activity (PS exposure) and fibrin formation within the same experiment. The current

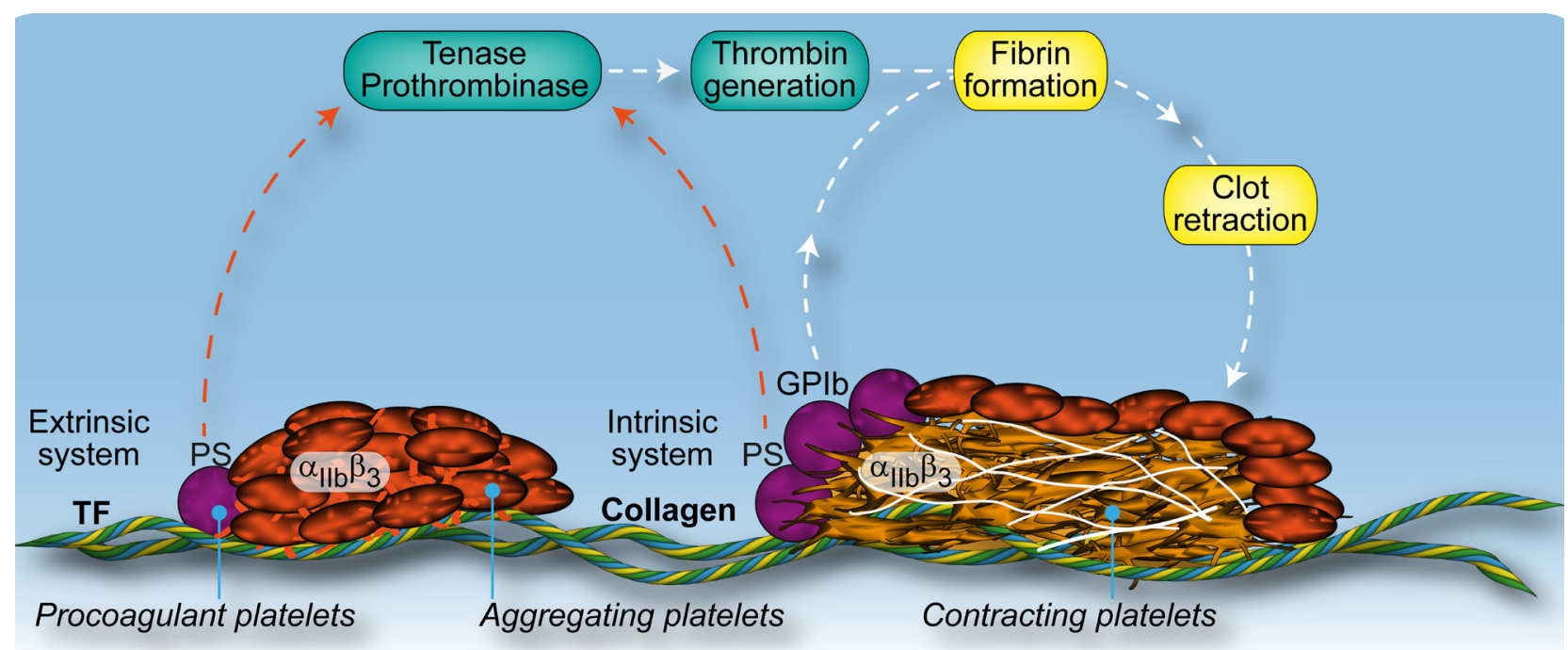

Figure 1. Platelet-based coagulation processes in a thrombus. The extrinsic and intrinsic coagulation systems are initiated after vascular disruption via tissue factor (TF) and collagen, respectively. Collagen-adhered platelets and patches of platelets in a thrombus expose PS, which serves as an efficient surface for assembly of the tenase (factors IXa/VIIIa) and prothrombinase (factors $\mathrm{Xa} / \mathrm{Va}$ ) complexes, resulting in thrombin generation and fibrin formation. At low shear rates, GPIb interaction with VWF mediates focal fibrin formation at the platelet surface via PS exposure. The aggregated platelets in a thrombus display activated $\mathrm{a}_{\mathrm{IIb}} \beta_{3}$ and accomplish clot retraction by contracting fibrin fibers in the presence of thrombin. 
methodology, using small-size chambers in combination with fluorescence microscopy, has revealed key roles of many platelet receptors and signaling proteins in the buildup of a thrombus and the formation of procoagulant platelets ${ }^{3}$. Table 1 provides an overview of published studies, where thrombus formation together with PS exposure is assessed under arterial shear conditions using blood from genetically modified mice. Table 2 shows corresponding data with human blood containing specific pharmacological inhibitors. Whereas the earlier literature concentrated on understanding the role of collagen receptor-induced $\mathrm{Ca}^{2+}$ fluxes in PS exposure, recent papers revealed the contribution of various downstream signaling events and of autocrine products secreted from platelet granules. In addition, adhesive receptors other than collagen receptors have been examined for a role in procoagulant activity.

\section{Collagen-induced $\mathrm{Ca}^{2+}$ signaling as the classical route}

At high, arterial wall-shear rate, platelet adhesion to von Willebrand factor (VWF) via glycoprotein Ib-V-IX (GPIb), and to collagen via the receptors glycoprotein VI (GPVI) and integrin $a_{2} \beta_{1}$ leads to strong activation and to the formation of a stable thrombus ${ }^{1,4}$. A subpopulation of the platelets in the thrombus, particularly those platelets in direct contact with the collagen and the platelets that are co-activated by thrombin assume a round morphology, which is accompanied by PS exposure and microparticle shedding ${ }^{5,6}$. In contrast to the aggregated platelets in the thrombus core, the PS-exposing cells lack activation of the fibrinogen receptor, integrin $\mathrm{a}_{\mathrm{IIb}} \beta_{3}{ }^{7}$. The main mechanism underlying PS exposure is a prolonged elevation in cytosolic $\mathrm{Ca}^{2+}$ level ${ }^{8,9}$. Hence, signaling molecules downstream of the GPVI-Fc receptor $y$ chain (FCRY) complex and involved in $\mathrm{Ca}^{2+}$ mobilization via phospholipase $\mathrm{CY}$ (PLCY) and phosphatidylinositol 3-kinase (PI3K) will contribute to PS exposure ${ }^{1}$. As indicated in Table 1, platelets from knockout mice deficient in several signaling molecules show a decreased ability to thrombus formation as well as PS exposure. These signaling proteins include the receptors GPIb (via VWF), GPVI, FCRY, $a_{2} \beta_{1}$ and $a_{I I b} \beta_{3}$; various adapter and scaffold proteins, e.g. linker for activated T-cells (LAT); effector proteins such as the PI3K isoforms $\alpha / \beta$, Src family kinases, and PLCY2. Inhibitory studies with human blood indicate a similar involvement of receptors (GPIb, GPVI, $a_{2} \beta_{1}, a_{\text {IIb }} \beta_{3}$ ) and signaling molecules (PI3K isoforms $a$ and $\beta$ and Src family kinases) in thrombus formation and PS exposure (Table 2). These findings support the concept that the reduced PS exposure is a direct consequence of diminished GPVI signaling to elevated $\mathrm{Ca}^{2+}$, rather than to a reduced platelet deposition per $\mathrm{se}^{9}$.

Collagen- and GPVI-dependent PS exposure is greatly enhanced by co-stimulation of platelets with thrombin (acting via the Gqa protein), at both static and flow conditions, an effect that is explained by the more prolonged $\mathrm{Ca}^{2+}$ mobilization in response to two agonists ${ }^{10}$. Other soluble agonists, in particular ADP, have been shown to enforce GPVI-induced $\mathrm{Ca}^{2+}$ signaling, PS exposure and thrombin generation, particularly via the $\mathrm{P}_{2} \mathrm{Y}_{12}$ receptors, and hence contribute to platelet procoagulant activity. Consequently, knock-out (mouse) or inhibition (human) of ${\mathrm{P} 2 \mathrm{Y}_{12}}_{12}$ resulted in a smaller thrombus size and decreased PS exposure ${ }^{11,12,13}$. However, thrombus formation not always coincides with PS exposure. For instance, the interaction of plasma-derived Gas6 with the platelet Gas6 receptors (TAM receptors), while enforcing integrin activation and contributing to thrombus stability, did not contribute to platelet PS exposure ${ }^{5,14}$.

\section{Insights into the role of elevated $\mathrm{Ca}^{2+}$}

Platelets contain well-developed $\mathrm{Ca}^{2+}$ entry mechanisms for inducing and maintaining high cytosolic $\mathrm{Ca}^{2+}$ levels ${ }^{15}$. Recent work has pointed to a role of the so-called store-operated and receptor-operated $\mathrm{Ca}^{2+}$ entry pathways in PS exposure. The integral membrane protein, stromal interaction molecule (STIM)1, has been identified as the central $\mathrm{Ca}^{2+}$ sensor in the platelet reticular membrane that regulates store-operated $\mathrm{Ca}^{2+}$ entry ${ }^{16}$. Via a direct coupling mechanism, STIM1 activates the $\mathrm{Ca}^{2+}$ entry channel Orai1. Deficiency in either STIM1 or Orai1 resulted in decreased thrombus formation and PS exposure on collagen (Figure $2 A)^{17,18}$. Interestingly, in the presence of 
Table 1. Mouse proteins and genes involved in platelet procoagulant activity and fibrin formation under high-shear flow conditions. Abbreviations: Ab, antibody; GOF, gain-of-function; LOF, loss-of-function; PS, phosphatidylserine, TS, thrombus size; = unchanged; $\uparrow$ increased; $\downarrow$ decreased.

\begin{tabular}{|c|c|c|c|c|}
\hline Target protein & Perturbation & $\begin{array}{l}\text { Effect on thrombus } \\
\text { formation }\end{array}$ & $\begin{array}{l}\text { Effect on } \\
\text { coagulation }\end{array}$ & Reference \\
\hline \multicolumn{5}{|c|}{ Platelet receptors and membrane proteins } \\
\hline $\mathrm{Axl}$ & $\left.A x\right|^{-1-}$ & TS $\downarrow$ (instability) & $\mathrm{PS}=$ & 14 \\
\hline Cyclophilin D & Ppid $\%$ & $\mathrm{TS} \uparrow$ & PS $\downarrow$ & 7 \\
\hline Glycoprotein Ib & $\mathrm{p} 0 \mathrm{p} / \mathrm{B} \mathrm{Ab}$ & TS $\downarrow$ & PS $\downarrow$ & 82 \\
\hline Glycoprotein VI & Fcer1g $\%$, JAQ1 Ab & TS $\downarrow$ (no thrombi) & PS $\downarrow$ & $34,83,84$ \\
\hline Gqa & Gnaq $\%$ & TS $\downarrow$ & PS $\downarrow$ & 83 \\
\hline Integrin $a_{2} \beta_{1}$ & $a_{2}$-null, $\beta_{1}$-null & $\mathrm{TS} \downarrow$ & PS $\downarrow$ & $83-85$ \\
\hline Integrin $\mathrm{a}_{\mathrm{IIb}} \beta_{3}$ & JON/A mAb & TS $\downarrow$ & PS $\downarrow$ & 82 \\
\hline LAT & Lat null, Lat ${ }^{+/}$, Lat ${ }^{-/}$ & TS $\downarrow$ & PS $\downarrow$ & 33,34 \\
\hline Mer & $\mathrm{Mer}^{-1-}$ & TS $\downarrow$ (instability) & $\mathrm{PS}=$ & 14 \\
\hline Orai1 & Orai1 $\%, 2-A P B$ & TS $\downarrow$ & PS $\downarrow$, fibrin $\downarrow$ & 17,18 \\
\hline $\mathrm{P}_{2} \mathrm{Y}_{1}$ & MRS-2179 & TS $\downarrow$ & PS $\downarrow$ & 83 \\
\hline $\mathrm{P} 2 \mathrm{Y}_{12}$ & P2yr12\%, cangrelor & TS $\downarrow$ & PS $\downarrow$ & 12 \\
\hline STIM1 & $\begin{array}{l}\text { Stim } 1 \% \\
\text { Stim1 (LOF) }\end{array}$ & $\begin{array}{l}\text { TS } \downarrow \\
\text { TS }=\end{array}$ & $\begin{array}{l}\text { PS } \downarrow \text {, fibrin } \downarrow \\
\text { PS }\end{array}$ & $\begin{array}{l}17 \\
23\end{array}$ \\
\hline Tyro3 & Tyro3\% & TS $\downarrow$ (instability) & $\mathrm{PS}=$ & 14 \\
\hline \multicolumn{5}{|c|}{ Platelet signaling proteins (kinases) } \\
\hline PI3 kinases & Pik3r1\%, wortmannin & TS $\downarrow$ & PS $\downarrow$ & 27 \\
\hline PI3 kinase $a / \beta$ & PIK-75, TGX-221, Pik3r2 (LOF) & TS $\downarrow$ & PS $\downarrow$ & $27,48,86$ \\
\hline Protein kinase $\mathrm{Ca}$ & Prkca ${ }^{-}$ & TS $\downarrow$ & PS $\downarrow$ & 87,88 \\
\hline Protein kinase $C \beta$ & $P r k c b^{-/}$ & TS $\downarrow$ (no thrombi) & PS $\downarrow$ & 87 \\
\hline Protein kinase $\mathrm{C} \delta$ & Prkcd ${ }^{-1-}$ & $\mathrm{TS} \uparrow$ & $\mathrm{PS}=$ & 87 \\
\hline Protein kinase $\mathrm{C} \theta$ & Prkcq ${ }^{-/}$ & $\mathrm{TS} \uparrow / \downarrow$ & $\mathrm{PS} \uparrow$ & 26,87 \\
\hline Protein kinase D2 & $P k d 2^{-}$ & TS $\downarrow$ & $\mathrm{PS}=$ & 89 \\
\hline Src family kinases & PD0173952 & TS $\downarrow$ (no thrombi) & PS $\downarrow$ & 33,85 \\
\hline \multicolumn{5}{|c|}{ Platelet signaling proteins (non-kinases) } \\
\hline a-Granules & Nbeal2 $\%$ & $\mathrm{TS} \downarrow$ & PS $\downarrow$ & 30 \\
\hline CalDAG-GEF1 & Rasgrp $\%$ & TS $\downarrow$ & PS $\downarrow$ & 23 \\
\hline Calpain-1 & Capn1\% & $\mathrm{TS}=$ & PS $\downarrow$ & 7 \\
\hline CLP36 & Clp36 (LOF) & $\mathrm{TS} \uparrow$ & $\mathrm{PS} \uparrow$ & 31 \\
\hline Gas6 & Gas6 $\%$ & TS $\downarrow$ (instability) & $\mathrm{PS}=$ & 14 \\
\hline Phospholipase $\mathrm{C}_{2} 2$ & $\begin{array}{l}\text { Plcg2 (GOF), } \\
\text { Plcg2 +/, Plcg2-- }\end{array}$ & $\begin{array}{l}\mathrm{TS} \uparrow \\
\mathrm{TS} \downarrow\end{array}$ & $\begin{array}{l}\mathrm{PS} \uparrow \\
\mathrm{PS} \downarrow\end{array}$ & $\begin{array}{l}90 \\
34\end{array}$ \\
\hline Phospholipase D1 & $P l d^{-/-}$ & TS $\downarrow$ & PS $\downarrow$ & 54 \\
\hline \multicolumn{5}{|l|}{ Plasma proteins } \\
\hline Factor XI & $F 11^{\%}$ & TS $\downarrow$ & PS $\downarrow$, fibrin $\downarrow$ & 34 \\
\hline Factor XII & $\mathrm{F} 12^{\%}, \mathrm{CTI}$ & TS $\downarrow$ & PS $\downarrow$, fibrin $\downarrow$ & 34 \\
\hline Factor VII & FVIIai & $\mathrm{TS} \downarrow$ & PS $\downarrow$, fibrin $\downarrow$ & 34 \\
\hline (Pro)thrombin & melagatran & $\mathrm{TS} \downarrow$ & PS $\downarrow$, fibrin $\downarrow$ & 35 \\
\hline VWF & saratin & $\mathrm{TS} \downarrow$ & $\mathrm{PS} \downarrow$ & 33,82 \\
\hline
\end{tabular}


Table 2. Human proteins involved in platelet procoagulant activity and fibrin formation under high-shear flow conditions. Abbreviations as for Table 1

\begin{tabular}{|c|c|c|c|c|}
\hline Target protein & Intervention & Effect on thrombus & $\begin{array}{l}\text { Effect on coagu- } \\
\text { lation }\end{array}$ & Reference \\
\hline \multicolumn{5}{|c|}{ Platelet receptors and membrane proteins } \\
\hline Glycoprotein Ib & 6B4, 12G1 Abs & TS $\downarrow$ & PS $\downarrow$ & 91 \\
\hline Glycoprotein VI & $9012,10 \mathrm{~B} 12 \mathrm{Abs}$ & TS $\downarrow$ (no throm- & PS $\downarrow$ & $11,92,93$ \\
\hline Integrin $a_{2} \beta_{1}$ & 6F1, 15D7 Abs & bi) & PS $\downarrow$ & $85,92-94$ \\
\hline Integrin $a_{\mathrm{IIb}} \beta_{3}$ & agrastat, latrofiban & TS $\downarrow$ (no thrombi) & PS $\downarrow$ & 85,94 \\
\hline Orai1 & $2-A P B$ & $\mathrm{TS} \downarrow$ & PS $\downarrow$ & 18 \\
\hline $\mathrm{P} 2 \mathrm{Y}_{12}$ & ticagrelor, cangrelor & TS $\downarrow$ (instability) & PS $\downarrow$, fibrin $\downarrow$ & $11,12,48$ \\
\hline \multicolumn{5}{|c|}{ Platelet signaling proteins (kinases) } \\
\hline PI3 kinases & wortmannin & TS $\downarrow$ (instability) & $\mathrm{PS} \downarrow$ & 27,48 \\
\hline PI3 kinase $a / \delta$ & PIK-75, YM-024 & $\mathrm{TS} \downarrow$ & PS $\downarrow$ & 27 \\
\hline PI3 kinase $\beta$ & TGX-221 & TS $\downarrow$ & PS $\downarrow$ & 27 \\
\hline PI3 kinase $\mathrm{g}$ & AS-252424 & $\mathrm{TS}=$ & $\mathrm{PS}=$ & 27 \\
\hline PI3 kinase d & IC-87144 & $\mathrm{TS}=$ & $\mathrm{PS}=$ & 27 \\
\hline Protein kinase $\mathrm{C}$ & GF-109203X, & TS $\downarrow$ (no thrombi) & $\mathrm{PS} \uparrow$ & 25 \\
\hline Src family kinases & $\begin{array}{l}\text { RO-318425 } \\
\text { PD-0173952 }\end{array}$ & TS $\downarrow$ (no thrombi) & $\mathrm{PS} \downarrow$ & 85 \\
\hline
\end{tabular}

high thrombin concentrations, STIM and Orai1 appeared to be less important for PS exposure ${ }^{17}$. This was recently explained by the finding that thrombin-dependent $\mathrm{Ca}^{2+}$ signaling in part relies on receptor-operated $\mathrm{Ca}^{2+}$ entry via TRPC channels ${ }^{19}$.

The $\mathrm{Ca}^{2+}$ signal in procoagulant platelets is enhanced by the release of mitochondrial $\mathrm{Ca}^{2+}$ via mitochondrial permeability transition pore formation (Figure $2 \mathrm{~A}$ ) ${ }^{20}$. A key regulator in this pore formation is the mitochondrial localized peptidylprolylisomerase, cyclophilin $D^{21}$. Accordingly, GPVI-induced PS exposure is reduced in platelets from mice lacking cyclophilin D ${ }^{7}$. However, in these platelets ionomycin-induced PS exposure is unchanged ${ }^{22}$, designating that mitochondrial collapse as such is not a strict requirement for PS exposure.

Redundancies downstream of diacylglycerol and $\mathrm{Ca}^{2+}$

Isoforms of PLC produce two second messengers, diacylglycerol (DAG) and inositol-trisphosphate serving as an intracellular $\mathrm{Ca}^{2+}$ mobilizer. Recent investigations have examined the roles in platelet procoagulant activity of two sets of $\mathrm{Ca}^{2+}$ - and DAG-dependent effector proteins, which are activated downstream of PLCY2, namely CaIDAG-GEF1 and isoforms of protein kinase C (PKC). Interestingly, these effector proteins can also modulate the $\mathrm{Ca}^{2+}$ signal itself, in a not yet completely understood way (Figure 2A). Platelets from mice lacking CalDAG-GEF1 are greatly impaired in thrombus formation as well as PS exposure ${ }^{23}$. However, the situation for PKC is more complex as described next.

Human and mice platelets express both the conventional PKC isoforms $a$ and $\beta$ and the novel PKC isoforms $\delta$ and $\theta{ }^{24}$. In human blood, addition of a general inhibitor for all PKC isoforms resulted in greatly abolished thrombus formation, but in increased PS exposure of the remaining adhered platelets ${ }^{25}$. Studies with knockout mice indicated a positive role in both thrombus formation and PS exposure of the two conventional PKC isoforms $a / \beta$, whereas the two novel PKC isoforms $\delta / \theta$ were inhibitory ${ }^{26,27}$. The precise phosphorylation events through which these PKC isoforms operate are still unclear ${ }^{24}$. Jointly, these data point to non-redundant and positive roles of CalDAG-GEF1 and $\mathrm{PKCa} / \beta$, and to antagonistic and negative roles of $\mathrm{PKC} / \theta$ in the regulation of 
thrombus buildup and PS exposure.

Calpain is another $\mathrm{Ca}^{2+}$-dependent effector protein controlling platelet activation. While deficiency in mouse calpain-1 had no effect on thrombus size, this resulted in a markedly lower PS exposure ${ }^{7,22}$.

\section{Additive roles of dense- and alpha-granule secretion}

Platelet dense $(\delta)$ and alpha (a) granule constituents are known to amplify platelet responses, such in agreement with the bleeding phenotype seen in patients lacking these granules ${ }^{28}$. Novel data indicate that granule secretion supports not only platelet aggregation, but also platelet procoagulant activity. The $\delta$-granules contain calcium, serotonin, ADP, ATP and polyphosphates. The observed impairment of collagen/thrombin-induced PS exposure of platelets deficient in $\mathrm{P}_{2} \mathrm{Y}_{12}$ or in the presence of P2Y ${ }_{12}$ inhibitors ${ }^{12,13}$, is explained by inability of secreted ADP to interact with this receptor on neighboring platelets and thereby diminished signaling to platelet procoagulant activity. Lack of $\delta$-granules is the underlying cause of the Hermansky-Pudlak bleeding disorder ${ }^{28}$. Mice deficient in one of the Hermansky-Pudlak genes (Hsp3) show an impaired thrombosis tendency in vivo, but PS exposure has not yet been assessed.

The a-granules store multiple proteins, several of which can support thrombus formation, e.g. VWF, fibrinogen, factor $\mathrm{V}$ and thrombospondin ${ }^{29}$. Mutations in the $\mathrm{Nbeal2}$ gene associate with gray platelet syndrome, a rare bleeding disorder characterized by macrothrombocytopenia and the absence of platelet a-granules. Mice lacking expression of the $\mathrm{Nbeal} 2 \mathrm{gene}$ have been reported to be decreased in both thrombus formation and PS exposure ${ }^{30}$. These mice provide an excellent tool for further determination of the roles of a-granule proteins in hemostasis, thrombosis and inflammation.

Secretion of both a- and $\delta$-granules is increased in CLP36-deficient mice, thus explaining a larger thrombus size and higher PS exposure under flow ${ }^{31}$. CLP36 is an adaptor protein that binds to a-actinin-1 and associates with actin filaments and stress fibers ${ }^{32}$. Jointly, these findings seem to indicate that both types of secretion granules have additive roles to PS exposure along with thrombus formation (Figure 2A).

\section{Regulation of platelet-dependent thrombin generation and fibrin formation}

Platelet membranes with exposed PS provide an efficient surface for assembly of the tenase (factors IXa, VIIIa) and prothrombinase (factors Xa, Va) complexes, accomplishing high thrombin generation and subsequent fibrin formation ${ }^{1}$. Interestingly, genetic ablation or pharmacological inhibition of factors of either the extrinsic (VII) or the intrinsic (XII, XI) coagulation pathways diminished thrombin generation under stasis (Tables 3-4). Several of the signaling proteins that contribute to GPVI-induced PS exposure under flow have also been shown to control GPVI-mediated thrombin generation under stasis, as depicted for GVPI, PI3K $\beta$, Syk, Orai1, STIM1 and P2Y ${ }_{12}$.

Platelets bind almost all coagulation factors, including thrombin and fibrin ${ }^{8}$. At high shear rate in the presence of tissue factor, fibrin is predominantly confined to the core of platelet thrombi, which implies platelet support of the extrinsic coagulation system, triggered by the tissue factor/ factor VIIa complex ${ }^{33}$. Studies carried out in the absence of tissue factor also point to a role of the intrinsic coagulation system via factors XI and XII. In flow studies on collagen with recalcified blood, collagen fibers were found to play a dual role, i.e. as a ligand for GPVI resulting in platelet activation, and as a substrate for the binding and activation of factor XII ${ }^{34}$. Consequently, deficiency in factor XII or XI resulted in a decreased thrombus size, reduced PS exposure, and delayed fibrin formation (Table 1). Inhibition of thrombin was even more suppressive ${ }^{35}$. On the other hand, signaling downstream of $\mathrm{P}_{2} \mathrm{Y}_{12}$ amplified these processes ${ }^{12,}{ }^{13}$. Factor XIIa may also play another role, i.e. by directly binding to fibrin and regulating clot structure ${ }^{36}$. Jointly, these findings point to involvement of both the intrinsic and extrinsic coagulation systems in thrombin generation and ensuing fibrin formation at high-shear flow conditions. Furthermore, formed thrombin generates a 

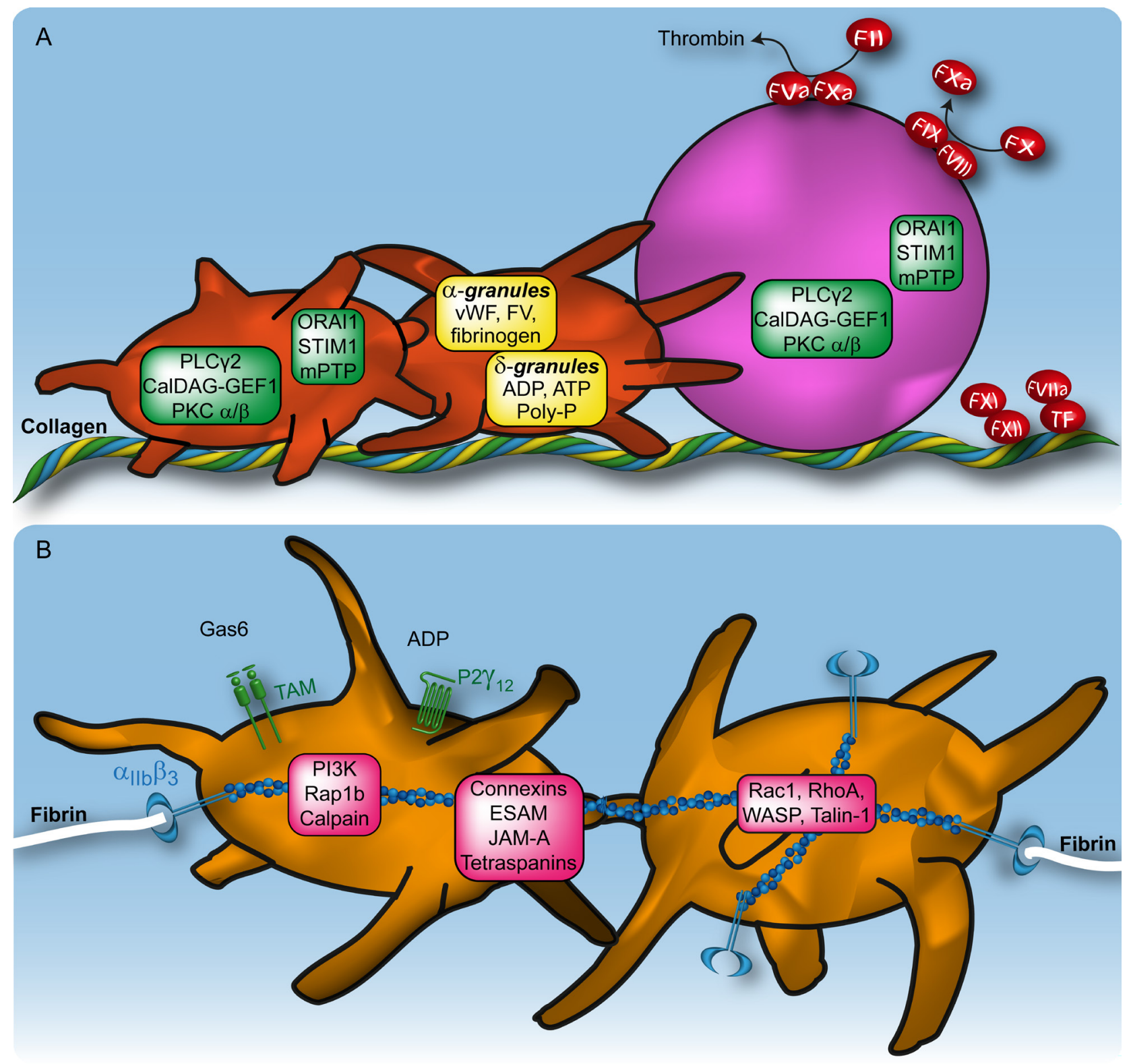

Figure 2. Signaling pathways implicated in platelet-dependent procoagulant activity, thrombin generation and clot retraction. (A) Platelets on collagen signal to second messenger generation (PLCg2, CalDAG-GEF1, PKC isoforms) and $\mathrm{Ca}^{2+}$ mobilization combined with store-operated $\mathrm{Ca}^{2+}$ entry (STIM1, Orai1) and release of mitochondrial $\mathrm{Ca}^{2+}$ via mPTP formation, resulting in sustained high cytosolic $\mathrm{Ca}^{2+}$ levels, secretion and PS exposure. These processes are supported by a- and d-granule constituents. The PS-exposing platelets show procoagulant activity by catalyzing tenase and prothrombinase activities (see also Figure 1). (B) Continuous inside-out signaling via PI3K, Rap1b and calpain leads to sustained $\mathrm{a}_{\mathrm{IIb}} \beta_{3}$ activation, enabling integrin outside-in mediated actin cytoskeleton reorganization via Rac1, RhoA, WASP and talin resulting in clot retraction. Homotypic interactions via connexins, ESAM and JAM-A differentially regulate clot retraction, while tetraspanins may directly interact with $\mathrm{a}_{\mathrm{IID}} \beta_{3}$.

positive feed-forward loop to enhanced PS exposure via activation of the PAR receptors on platelets ${ }^{8}$.

Classically, insufficiently controlled coagulation is regarded as key mediator of thrombosis under venous shear conditions. Recently, several insights also point to a role of platelets, in interplay with VWF and coagulation factors, in the thrombotic process under flow at low, venous wall-shear rates. Under coagulant conditions and low shear rate (implying accumulation of thrombin), a fibrin network is formed on the platelet surface with an outward-directed star-like appearance ${ }^{37}$. This focal fibrin formation relies on the VWF-GPIb axis, causing rises in cytosolic $\mathrm{Ca}^{2+}$ and PS exposure 
37. The signaling mechanism may involve GPIb-dependent changes of the actin cytoskeleton 38,39 . Interestingly, the fibrin fibers incorporate thrombin that is relocating from PS-exposing and factor Xa-binding platelets ${ }^{40}$.

The role of neutrophils in murine venous thrombosis models is a research topic that recently received much attention. It was reported that neutrophils, which are trapped in murine veins subjected to partial stasis, produce neutrophil extracellular traps (NETs), i.e. large extracellular DNA strands fibers surrounded by histones and neutrophil antimicrobial proteins ${ }^{41}$. In venous and microvascular thrombosis models, NETs formation can be a stimulus and scaffold for the formation of large thrombi of platelets, fibrin and erythrocytes ${ }^{41,42}$. Furthermore, NETs appeared to be formed in the vasculature during sepsis, where they may help to reduce microbial infections ${ }^{43,44}$. The underlying mechanisms of platelet responses to neutrophils and NETs have to be elucidated.

\section{Towards understanding platelet-dependent fibrin clot retraction}

In hemostasis, the process of platelet-dependent clot retraction provides a means to tighten wound edges after clotting ${ }^{45}$. Especially under conditions of coagulation and fibrin formation, thrombi that are formed under flow can undergo a mechanism of retraction, which can be considered as equivalent to clot retraction ${ }^{8}$. However, clot retraction as such has mostly been examined under static conditions, e.g. by determining the condensation of a platelet/fibrin clot in thrombin-stimulated platelet-rich-plasma. In general, clot retraction is regarded as a platelet response evoked by $a_{\mathrm{II}} \beta_{3}$ inside-out signaling, thus implying dependency of (thrombin-induced) $a_{\text {IIb }} \beta_{3}$ activation and binding of fibrinogen to platelets ${ }^{46}$. Table 3 provides an overview of published effects of genetic knockout in mouse on clot retraction, while Table 4 gives corresponding data for human blood. Below we describe new insights into the molecular mechanisms with emphasis on integrin inside-out and outside-in signaling, homotypic platelet-platelet interactions, and the role of the actin cytoskeleton.

\section{Enforcement of $a_{I I b} \beta_{3}$ inside-out signaling}

While clot retraction for long has been recognized as a process that reflects integrin outside-in signaling, new data provide insight into the underlying signaling events in platelets via activated integrins. The ADP receptor $\mathrm{P}_{2} \mathrm{Y}_{12}$ prolongs integrin activation and platelet aggregation ${ }^{47}$. Continued signaling via this receptor (via Gi and PI3K $\beta$ ) maintains $a_{I I b} \beta_{3}$ in the active conformation and thus prevents platelet disaggregation ${ }^{48,49}$. Likely, similar signaling mechanisms are implicated in clot retraction, since mouse platelets lacking integrin $\beta_{3}$ or PI3K $\beta$ are impaired in this process (Table 3 , Figure 2B).

An enforcement loop that acts in a similar, though weaker way is that of (plasma-derived) Gas6 interacting with the platelet Gas6 receptors, also called TAM receptors (Tyro3, Axl and Mer). Activation of these receptors by Gas6 supports platelet aggregation and tyrosine phosphorylation of the $\beta_{3}$ integrin chain, again in a PI3K-dependent way ${ }^{47,50}$. Overall, TAM receptor signaling can enforce the activation of $\mathrm{a}_{\mathrm{IIb}} \beta_{3^{\prime}}$ and thus help to prevent platelet disaggregation. Platelets from mice deficient in one of the TAM receptors have a decreased ability to clot retraction, which supports a role for Gas6 and its receptors in integrin outside-in signaling (Figure 2B) ${ }^{50}$.

Calcium also play a central role in integrin activation via the $\mathrm{Ca}^{2+}$ - and DAG-dependent effector proteins CalDAG-GEF1 and isoforms of protein kinase C (PKC), which are activated downstream of PLCY2. Activation of Rap1b - via CALDAG-GEF or PKC - promotes the assembly of an activation complex with RIAM (Rap1 interacting adaptor molecule) and talin. Cleavage of talin mediated by RIAM, calpain and phosphatidylinositol 4,5-biphosphate (PIP ${ }_{2}$ ) enables talin binding to the integrin $\beta_{3}$ chain, thereby activating the integrin and enabling ligand binding ${ }^{51}$. An additional level of regulation is provided by phospholipase D (PLD) 1 and 2. Both isoforms are present in platelets and translocate to the plasma membrane during platelet activation and produce phosphatidic acid to stimulate the production of $\mathrm{PIP}_{2}{ }^{52}, 53$. Mice deficient in PLD1 display impaired thrombus formation and PS exposure due to impaired integrin $a_{\mathrm{IIb}} \beta_{3}$ inside-out signaling ${ }^{54}$. 
Table 3. Mouse proteins and genes involved in platelet-dependent thrombin generation and clot retraction under stasis. Abbreviations: see Table 1. In addition: CR clot retraction; TG thrombin generation; * also involved in PS exposure, see Table 1.

\begin{tabular}{|c|c|c|c|}
\hline Target protein & Perturbation & $\begin{array}{l}\text { Effect on } \\
\text { coagulation }\end{array}$ & Reference \\
\hline \multicolumn{4}{|c|}{ Platelet receptors and membrane proteins } \\
\hline $\mathrm{Axl}$ & $A x^{-1-}$ & $\mathrm{CR} \downarrow$ & 50 \\
\hline Connexin 37 & Gja4\% & $\mathrm{CR} \downarrow$ & 59 \\
\hline Connexin 40 & Gja5\%, ${ }^{40}$ Gap27 & $\mathrm{CR} \downarrow$ & 60 \\
\hline Cyclophilin D & Ppid ${ }^{-1}$ & $\mathrm{CR} \downarrow$ & 22 \\
\hline ESAM & $E S A M^{-1-}$ & $\mathrm{CR} \downarrow$ & 67 \\
\hline Galectin-1 & Lgals1\%- & $\mathrm{CR} \downarrow$ & 55 \\
\hline Integrin $a_{\mathrm{TH}} \beta_{3}$ & $\beta_{3}$-null & $\mathrm{CR} \downarrow$ & 95 \\
\hline JAM-A & $F 11 r^{\prime-}$ & $\mathrm{CR} \uparrow$ & 61 \\
\hline Mer & $\mathrm{Mer}^{--}$ & $\mathrm{CR} \downarrow$ & 50 \\
\hline Orai1 & Orai1\% & TG $\downarrow$ & 17 \\
\hline$P 2 Y_{12}$ & cangrelor, ticagrelor & TG $\downarrow$ & 11-13 \\
\hline PECAM-1 & Pecam1 ${ }^{\%}$ & $C R=$ & 72 \\
\hline STIM1 & Stim $1 \%$ & TG $\downarrow$ & 17 \\
\hline Tetraspanin 32 (TSSC6) & Tspan32\%- & $\mathrm{CR} \downarrow$ & 70 \\
\hline Tetraspanin CD151 & $C d 151^{\%}$ & $\mathrm{CR} \downarrow$ & 71 \\
\hline TMEM16F (anoctamin 6) & Ano $6^{\%}$ & TG $\downarrow$ & 96 \\
\hline Tyro3 & Tyro3 $\%$ & $\mathrm{CR} \downarrow$ & 50 \\
\hline \multicolumn{4}{|c|}{ Platelet signaling proteins (kinases/phosphatases) } \\
\hline AMPKa2 & Prkaa2\% & $\mathrm{CR} \downarrow$ & 97 \\
\hline PDK1 & Pdpk1\%, Pdpk1 (LOF) & $\mathrm{CR} \downarrow$ & 98 \\
\hline PI3 kinase & LY-294002 & $\mathrm{CR} \downarrow$ & 76 \\
\hline PI3 kinase $\beta$ & Pik3cb-null, TGX-221 & $\mathrm{CR} \downarrow$ & 75 \\
\hline Protein phosphatase 1 & $P p 1 \mathrm{cg}^{-1}$ & $\mathrm{CR} \downarrow$ & 99 \\
\hline PTP1B & Ptp $1 b^{-/-}$ & $\mathrm{CR}=/ \downarrow$ & 100,101 \\
\hline \multicolumn{4}{|l|}{ Cytoskeleton linked proteins } \\
\hline Rac1 & EHT-1864 & $\mathrm{CR} \downarrow$ & 102 \\
\hline Rap1b & $R a p 1 b^{-/}$ & $\mathrm{CR} \downarrow$ & 103 \\
\hline RhoA & Rhoa\% & $\mathrm{CR} \downarrow$ & 79 \\
\hline Talin-1 & TIn1-null & $\mathrm{CR} \downarrow$ & 104 \\
\hline WASP & Wasp ${ }^{1-}$ & $\mathrm{CR} \downarrow$ & 81 \\
\hline \multicolumn{4}{|c|}{ Platelet signaling proteins (other) } \\
\hline Calpain & Capn $1^{-}$ & $\mathrm{CR} \downarrow$ & 100 \\
\hline $\mathrm{Cbl}$ & $C b^{-1}, C b l(L O F)$ & $\mathrm{CR} \downarrow$ & 105 \\
\hline Gas6 & $\operatorname{Gas6}^{-1}$ & $T G=$ & 14 \\
\hline Lnk & $\operatorname{Sh} 2 b 3^{-1}$ & $\mathrm{CR} \downarrow$ & 106 \\
\hline mTOR & rapamycin & $\mathrm{CR} \downarrow$ & 107 \\
\hline NF-kB & BAY-117082 & $\mathrm{CR} \downarrow$ & 108 \\
\hline PER2 & Per2-null & $\mathrm{CR} \downarrow$ & 109 \\
\hline Phosphodiesterase 3 & milrinone & TG $\downarrow$ & 110 \\
\hline Phospholipase Cy2 & $\mathrm{PlCq}^{-1-}$ & $\mathrm{CR} \downarrow$ & 111 \\
\hline SHIP1 (PIP ${ }_{3}$ phosphatase) & Inpp55-null & $\mathrm{CR} \downarrow$ & 112 \\
\hline \multicolumn{4}{|l|}{ Plasma proteins } \\
\hline Factor XII & $\mathrm{F} 12^{\%}, \mathrm{CTI}$ & TG $\downarrow$ & 34 \\
\hline Factor XI & $\mathrm{F} 11^{\%}$ & TG & 34 \\
\hline Factor VII & FVIIai & TG $\downarrow$ & 34 \\
\hline (Pro)thrombin & melagatran & TG $\downarrow$ & 35 \\
\hline
\end{tabular}


Table 4. Human proteins involved in platelet-dependent thrombin generation and clot retraction coagulation under stasis For abbreviations, see Table 3.

\begin{tabular}{|c|c|c|c|}
\hline Target protein & Intervention & $\begin{array}{l}\text { Effect on } \\
\text { coagulation }\end{array}$ & Reference \\
\hline \multicolumn{4}{|c|}{ Platelet receptors and membrane proteins } \\
\hline IP receptor & prostaglandin $\mathrm{E}_{1}$ & TG $\downarrow$ & 113,114 \\
\hline G13 protein & mSRI peptide & $\mathrm{CR} \downarrow$ & 115 \\
\hline Gap junctions & carbenoxolone, Gap27, 18ß-GA & $\mathrm{CR} \downarrow$ & 59 \\
\hline Glycoprotein VI & $9012 \mathrm{Ab}$ & TG $\downarrow$ & 113,114 \\
\hline Integrin $a_{2} \beta_{1}$ & $6 \mathrm{~F} 1 \mathrm{Ab}$ & $\mathrm{TG} \downarrow$ & 113 \\
\hline Integrin $\mathrm{a}_{\mathrm{II}} \beta_{3}$ & Glanzmann patients, abciximab & $\mathrm{CR} \downarrow, \mathrm{TG} \downarrow$ & $113,114,116$ \\
\hline Orai1 & $2-\mathrm{APB}$ & $\mathrm{TG} \downarrow$ & 18 \\
\hline $\mathrm{P} \mathrm{Y}_{12}$ & cangrelor, ticagrelor & TG $\downarrow$ & $12,113,114,117$ \\
\hline \multicolumn{4}{|c|}{ Platelet signaling proteins (kinases) } \\
\hline PI3 kinase $\beta$ & TGX-221 & TG $\downarrow$ & 117 \\
\hline SFK & PD0173952 & $\mathrm{CR} \downarrow$ & 78 \\
\hline Syk & Syk inhibitor II & TG $\downarrow$ & 118 \\
\hline \multicolumn{4}{|c|}{ Platelet signaling proteins (non-kinases) } \\
\hline $\mathrm{Cbl}$ & OXSI-2, PP2 & $\mathrm{CR} \downarrow$ & 105 \\
\hline Cyclooxygenase & aspirin & TG $\downarrow$ & 113 \\
\hline WASP & WAS patients & $\mathrm{CR} \downarrow$ & 81 \\
\hline \multicolumn{4}{|l|}{ Plasma proteins } \\
\hline Factor XII & Corn trypsin inhibitor & TG $\downarrow$ & 34 \\
\hline Factor VII & FVIIai & TG $\downarrow$ & 34 \\
\hline
\end{tabular}

Fibrin clot retraction is also reduced in these mice, similarly as in mice lacking Rap1b or talin [54, 103, 104].

Recently, galectin- 1 has been recognized as a new platelet agonist perhaps acting by direct activation of $\mathrm{a}_{\mathrm{IL}} \beta_{3}$. Galactin-1 binding to platelets evokes common activation signals, such as $\mathrm{Ca}^{2+}$ mobilization, phosphorylation of PLCY2, Syk of and mitogen-activated protein kinases ${ }^{55}$. Mice lacking galectin-1 were found to be impaired in clot retraction, likely because of diminished outside-in signaling.

Homotypic interactions regulating $a_{I I b} \beta_{3}$ activity

Platelets contain a whole repertoire of receptors and signaling molecules that regulate the formation of tight platelet-platelet contacts and can trigger contact-dependent signaling events via homotypic interactions ${ }^{56}$. Among these are proteins forming gap junctions via the inter-cellular coupling of two hemichannels between adjacent cells ${ }^{57,58}$. Two members of the family of connexins - implicated in hemichannel formation - i.e. connexins 37 and 40, are highly expressed in platelets. Knockout (mouse) and inhibitor (human) studies indicated that both connexins participate in platelet-dependent clot retraction ${ }^{59,60}$. Surprisingly, however, these connexins also appeared to contribute to other activation processes, such as platelet aggregation, integrin activation, $\mathrm{Ca}^{2+}$ signaling and a-granule secretion ${ }^{59}$. Hence, it seems that connexins have an overall role in the platelet activation process, rather than a specific role in tight junction formation during clot retraction. Clearly, more research is needed to elucidate the precise action mechanisms of platelet connexins. 
Junctional adhesion molecule (JAM)-A is another established molecule exhibiting homotypic interactions in platelets ${ }^{61,62}$. Recently, JAM-A was identified as a negative regulator of platelet activation and, hence, of thrombus formation ${ }^{61}$. Along the same line, integrin outside-in signaling and clot retraction were increased in mouse platelets lacking JAM-A. Earlier data suggested that JAM-A acts as a co-receptor for stimulatory antibodies via interaction with the FcyRIIA receptor on human platelets ${ }^{63,64}$. The mechanism proposed is suppression by JAM-A of integrin outside-in signaling through downstream protein kinases, such as Src, Syk and focal adhesion kinase ${ }^{65,66}$. An earlier suggestion, that JAM-A interferes with fibrinogen binding to the activated integrin, could not be confirmed in recent work ${ }^{61}$.

In spite of the examples above, integrin outside-in signaling and clot retraction are not always affected in the same ways. In platelets lacking endothelial cell specific adhesion molecule (ESAM), spreading on immobilized fibrinogen (reflecting outside-in signaling) was unaffected, while fibrin clot retraction was delayed ${ }^{67}$. Similarly to connexins and JAM-A, also ESAM is considered to cluster platelets via homotypic interactions.

Platelet integrins form multi-molecular complexes with other membrane proteins such tetraspanins. Reported are high expressions of tetraspanin-32, CD151, CD9 and CD63 ${ }^{68}$. It has been suggested that tetraspanins support integrins in the regulation of contact-dependent signaling ${ }^{69}$. Although the precise molecular mechanism is still unclear, tetraspanin interaction with integrins is likely, since deficiency in tetraspanin-32 or CD151 was found to lead to impaired clot retraction ${ }^{70}$, 71 .

Another 'difficult' membrane protein in terms of platelet function is PECAM-1. Platelets from mice lacking PECAM-1 have been reported as no more than slightly impaired in agonist-induced responses, with unchanged clot retraction ${ }^{72}$. This is in contrast to an earlier detected inhibitory role of PECAM-1 in human platelet function ${ }^{73,74}$. The reason for this discrepancy is unclear. Taken together, the various platelet proteins implicated in homotypic interactions seem to contribute in a balanced way (positive and negative) to platelet clot retraction, often in a similar way as to integrin activation (Figure 2B).

\section{Actin cytoskeleton rearrangement}

Traditionally, clot retraction is thought to be a thrombin- and fibrin-dependent process initiated by fibrin binding to $a_{\text {IIb }} \beta_{3}$. Subsequent actin and myosin cytoskeleton rearrangements generate contracting forces that are transmitted to the fibrin clot (Figure 2B) ${ }^{75,76}$. More recent findings point to the existence of a clot retraction mechanism involving Rho kinase and Src family kinases, which precedes the actin-dependent process 77,78 . In agreement with this, genetic ablation of RhoA was found to diminished clot retraction ${ }^{79}$. Furthermore, integrin-dependent phosphorylation by Src and Syk kinases of the ubiquitin ligase $\mathrm{Cbl}$ leads to the recruitment of $\mathrm{SH} 2$ domain containing proteins that mediate platelet spreading and clot retraction ${ }^{80}$.

The Wiskott-Aldrich syndrome, caused by a deficiency in the Wiskott-Aldrich syndrome protein (WASP), is accompanied with thrombocytopenia and a small platelet size ${ }^{28}$. Platelets from mice deficient in WASP are decreased in ability to clot retraction, likely because of abnormal actin filament formation ${ }^{81}$. A similar phenotype is observed in platelets from Wiskott-Aldrich syndrome patients.

In human (thrombin-stimulated) platelet-rich plasma, weakened clot retraction is measured upon pharmacological inhibition of $\mathrm{a}_{\mathrm{IIb}} \beta_{3}$, G13 protein, $\mathrm{Cbl}$ or gap junction proteins (Table 4). Taken together, the findings with mouse and human platelets may indicate that especially impairments in sustained integrin activation, in platelet-platelet contacts or in actin filament formation lead to diminished clot retraction. Whether deficient clot retraction is a marker for impaired hemostasis (bleeding), is an attractive hypothesis. However, this has not systematically been studied. 


\section{Conclusion}

Studies with both genetically modified mice and pharmacological inhibitors indicate that, in collagen-adhered platelets, in part common signaling pathways lead to PS exposure (procoagulant activity), generation of thrombin and fibrin, and retraction of the fibrin clot. However, signaling events causing prolonged $\mathrm{Ca}^{2+}$ elevation particularly stimulate PS exposure and thrombin generation, whereas integrin-dependent signaling events rather enforce fibrin clot retraction. Contact-dependent signaling pathways, triggered by homotypic platelet-platelet interactions, seem to act via the integrin route.

\section{Acknowledgements}

This work was supported by grants from the Center for Translational Molecular Medicine (INCOAG), the Netherlands Heart Foundation (2011T6) and ZonMW (MKMD 114021004).

\section{References}

1. Versteeg HH, Heemskerk JW, Levi M, Reitsma PH. New fundamentals in hemostasis. Physiol Rev 93, 327-358 (2013).

2. Monroe DM, Hoffman M. What does it take to make the perfect clot? Arterioscler Thromb Vasc Biol 26, 41-48 (2006).

3. Van Kruchten R, Cosemans JM, Heemskerk JW. Measurement of whole blood thrombus formation using parallel-plate flow chambers - a practical guide. Platelets 23, 229-242 (2012).

4. Jackson SP. The growing complexity of platelet aggregation. Blood 109, 5087-5095 (2007).

5. Cosemans JM, Angelillo-Scherrer A, Mattheij NJ, Heemskerk JW. The effects of arterial flow on platelet activation, thrombus growth, and stabilization. Cardiovasc Res 99, 342-352 (2013).

6. Munnix IC, Kuijpers MJ, Auger J, Thomassen CM, Panizzi P, Van Zandvoort MA, Rosing J, Bock PE, Watson SP, Heemskerk JW. Segregation of platelet aggregatory and procoagulant microdomains in thrombus formation: regulation by transient integrin activation. Arterioscler Thromb Vasc Biol 27, 2484-2490 (2007).

7. Mattheij NJ, Gilio K, Van Kruchten R, Jobe SM, Wieschhaus AJ, Chrishti AH, Collins P, Heemskerk JW, Cosemans JM. Dual mechanism of integrin $\mathrm{a}_{\mathrm{IIb}} \beta_{3}$ closure in procoagulant platelets. J Biol Chem 288, 13325-13336 (2013).

8. Heemskerk JW, Mattheij NJ, Cosemans JM. Platelet-based coagulation: different populations, different functions. J Thromb Haemost 11, 2-16 (2013).

9. Munnix IC, Cosemans JM, Auger JM, Heemskerk JW. Platelet response heterogeneity in thrombus formation. Thromb Haemost 102, 1149-1156 (2009).

10. Heemskerk JW, Kuijpers MJ, Munnix IC, Siljander PR. Platelet collagen receptors and coagulation. A characteristic platelet response as possible target for antithrombotic treatment. Trends Cardiovasc Med 15, 86-92 (2005).

11. Cosemans JM, Kuijpers MJ, Lecut C, Loubele ST, Heeneman S, Jnadrot-Perrus M, Heemskerk JW. Contribution of platelet glycoprotein VI to the thrombogenic effect of collagens in fibrous atherosclerotic lesions. Atherosclerosis 181, 19-27 (2005).

12. Nergiz-Ünal R, Cosemans JM, Feijge MA, Van der Meijden PE, Storey RF, Van Giezen JJ, Oude Egbrink MG, Heemskerk JW, Kuijpers MJ. Stabilizing role of platelet $P 2 Y_{12}$ receptors in shear-dependent thrombus formation on ruptured plaques. Plos One 5, e10130 (2010).

13. Van der Meijden PE, Feijge MA, Giesen PL, Huijberts M, van Raak LP, Heemskerk JW. Platelet P2Y receptors enhance signalling towards procoagulant activity and thrombin generation. A study with healthy subjects and patients at thrombotic risk. Thromb Haemost 93, 1128-1136 (2005).

14. Cosemans JM, Van Kruchten R, Olieslagers S, Schurgers LJ, Verheyen FK, Munnix IC, Waltenberger J, Angelillo-Scherrer A, Hoylaerts MF, Carmeliet P, Heemskerk JW. Potentiating role of Gas6 and Tyro3, Axl and Mer (TAM) receptors in human and murine platelet activation and thrombus stabilization. J Thromb Haemost 8, 1797-1808 (2010).

15. Varga-Szabo D, Braun A, Nieswandt B. STIM and Orai in platelet function. Cell Calcium 50, 270-278 (2011).

16. Varga-Szabo D, Braun A, Kleinschnitz C, Bender M, Pleines I, Pham M, Renné T, Stoll G, Nieswandt B. The calcium sensor STIM1 is an essential mediator of arterial thrombosis and ischemic brain infarction. J Exp Med 205, 1583-1591 (2008).

17. Gilio K, Van Kruchten R, Braun A, Berna-Erro A, Feijge MA, Stegner D, Van der Meijden PE, Kuijpers 
MJ, Varga-Szabo D, Heemskerk JW, Nieswandt B. Roles of platelet STIM1 and Orai1 in glycoprotein VI- and thrombin-dependent procoagulant activity and thrombus formation. J Biol Chem 285, 2362923638 (2010).

18. Van Kruchten R, Braun A, Feijge MA, kuijpers MJ, Rivera-Galdos R, Kraft P, Stoll G, Kleinschnitz C, Bevers EM, Nieswandt B, Heemskerk JW. Antithrombotic potential of blockers of store-operated calcium channels in platelets. Arterioscler Thromb Vasc Biol 32, 1717-1723 (2012).

19. Harper MT, Londõno JE, Quick K, Londõno JC, Flockerzi V, Philipp SE, Birnbaumer L, Freicel M, Poole AW . Transient receptor potential channels function as a coincidence signal detector mediating phosphatidylserine exposure. Sci Signal 6, ra50 (2013).

20. Choo HJ, Saafir TB, Mkumba L, Wagner MB, Jobe SM. Mitochondrial calcium and reactive oxygen species regulate agonist-initiated platelet phosphatidylserine exposure. Arterioscler Thromb Vasc Biol 32, 2946-2955 (2012).

21. Remenyi G, Szasz R, Friese P, Dale GL. Role of mitochondrial permeability transition pore in coated-platelet formation. Arterioscler Thromb Vasc Biol 25, 467-471 (2005).

22. Jobe SM, Wilson KM, Leo L, Raimondi A, Molkentin JD, Lentz SR, Di Paola J. Critical role for the mitochondrial permeability transition pore and cyclophilin D in platelet activation and thrombosis. Blood 111, 1257-1265 (2008).

23. Ahmad F, Boulaftali Y, Greene TK, Ouellette TD, Poncz M, Feske S, Bergmeier W. Relative contributions of stromal interaction molecule 1 and CaIDAG-GEFI to calcium-dependent platelet activation and thrombosis. J Thromb Haemost 9, 2077-2086 (2011).

24. Heemskerk JW, Harper MT, Cosemans JM, Poole AW. Unravelling the different functions of protein kinase $C$ isoforms in platelets. FEBS Lett 585, 1711-1716 (2011).

25. Strehl A, Munnix IC, Kuijpers MJ, Van der Meijden PE, Cosemans JM, Feijge MA, Nieswandt B, Heemskerk JW. Dual role of platelet protein kinase $C$ in thrombus formation: stimulation of pro-aggregatory and suppression of procoagulant activity in platelets. J Biol Chem 282, 7046-7055 (2007).

26. Hall KJ, Harper MT, Gilio K, Cosemans JM, Heemskerk JW, Poole AW. Genetic analysis of the role of protein kinase $\mathrm{Cq}$ in platelet function and thrombus formation. Plos One 3, e3277 (2008).

27. Gilio K, Munnix IC, Mangin P, Cosemans JM, Feijge MA, Van der Meijden PE, Olieslagers S, Chrzanowska-Wodnicka MB, Lilian R, Schoenwaelder S, Koyasu S, Sage SO, Jackson SP, Heemskerk JW. Non-redundant roles of phosphoinositide 3-kinase isoforms $a$ and $b$ in glycoprotein VI-induced platelet signaling and thrombus formation. J Biol Chem 284, 33750-33762 (2009).

28. Nurden AT, Freson K, Seligsohn U. Inherited platelet disorders. Haemophilia 18 Suppl 4, 154-160 (2012).

29. Blair P, Flaumenhaft R. Platelet a-granules: basic biology and clinical correlates. Blood Rev 23, 177189 (2009).

30. Deppermann C, Cherpokova D, Nurden P, Schulz JN, Thielmann I, Kraft P, Vögtle T, Kleinschnitz C, Dütting S, Krohne G, Eming SA, Nurden AT, Eckes B, Stoll G, Stegner D, Nieswandt B. Gray platelet syndrome and defective thrombo-inflammation in Nbeal2-deficient mice. J Clin Invest 123, 33313342 (2013).

31. Gupta S, Braun A, morowski M, Premsler T, Bender M, Nagy Z, Sickmann A, Hermanns HM, Bösl M, Nieswandt B. CLP36 is a negative regulator of glycoprotein VI signaling in platelets. Circ Res 111, 1410-1420 (2012).

32. Bauer K, Kratzer M, Otte M, De Quintana KL, Hagmann J, Arnold J, Eckerskorn C, Lottspeich F, Siess W. Human CLP36, a PDZ-domain and LIM-domain protein, binds to a-actinin-1 and associates with actin filaments and stress fibers in activated platelets and endothelial cells. Blood 96, 4236-4245 (2000).

33. Munnix IC, Strehl A, Kuijpers MJ, Auger JM, Van der Meijden PE, Van Zandvoort MA, Oude Egbrink MG, Nieswandt B, Heemskerk JW. The glycoprotein VI-phospholipase CY2 signaling pathway controls thrombus formation induced by collagen and tissue factor in vitro and in vivo. Arterioscler Thromb Vasc Biol 25, 2673-2678 (2005).

34. Van der Meijden PE, Munnix IC, Auger JM, Govers-Riemslag JW, Cosemans JM, Kuijpers MJ, Spronk HM, Watson SP, Renné T, Heemskerk JW. Dual role of collagen in factor XII-dependent thrombus formation. Blood 114, 881-890 (2009).

35. Kuijpers MJ, Munnix IC, Cosemans JM, Vlijmen BV, Reutelingsperger CP, Egbrink OG, Heemskerk. Key role of platelet procoagulant activity in tissue factor-and collagen-dependent thrombus formation in arterioles and venules in vivo differential sensitivity to thrombin inhibition. Microcirculation 15, 269282 (2008).

36. Konings J, Govers-Riemslag JW, Philippou H, Mutch NJ, Borissoff JI, Allan P, Mohan S, Tans G, Ten 
Cate H, Ariëns RA. Factor XIIa regulates the structure of the fibrin clot independently of thrombin generation through direct interaction with fibrin. Blood 118, 3942-3951 (2011).

37. Cosemans JM, Schols SE, Stefanini L, de Witt S, Feijge MA, Hamulyák K, Deckmyn H, Bergmeier W, Heemskerk JW. Key role of glycoprotein Ib/V/IX and von Willebrand factor in platelet activation-dependent fibrin formation at low shear flow. Blood 117, 651-660 (2011).

38. Fox JE. Identification of actin-binding protein as the protein linking the membrane skeleton to glycoproteins on platelet plasma membranes. J Biol Chem 260, 11970-11977 (1985).

39. Okita JR, Pidard D, Newman PJ, Montgomery RR, Kunicki TJ. On the association of glycoprotein Ib and actin-binding protein in human platelets. J Cell Biol 100, 317-321 (1985).

40. Berny MA, Munnix IC, auger JM, Schols SE, Cosemans JM, Panizzi P, Bock PE, Watson SP, McCarty OJ, Heemskerk JW. Spatial distribution of factor Xa, thrombin, and fibrin(ogen) on thrombi at venous shear. Plos One 5, e10415 (2010).

41. Fuchs TA, Brill A, Duerschmied D, Schatzberg D, Monesier M, Myers DD Jr, Wrobleski SK, Wakefield TW, Hartwig JH, Wagner DD. Extracellular DNA traps promote thrombosis. Proc Natl Acad Sci USA 107, 15880-15885 (2010).

42. von Bruhl ML, Stark K, Steinhart A, Chandraratne S, Konrad I, Lorenz M, Khndoga A, Tirniceriu A, Coletti R, Köllnberger M, byrne RA, Laitinen I, walch A, Brill A, pfeiler $S$, Manukyan D, Brun S, Lange $P$, Riegger J, Ware J, Eckart A, Haidari S, Rudelius M, Schulz C, Echtler K, Brinkmann V, Schwaiger M, Pressner KT, Wagner DD, Mackman N, Engelmann B, Massberg S. Monocytes, neutrophils, and platelets cooperate to initiate and propagate venous thrombosis in mice in vivo. J Exp Med 209, 819-835 (2012).

43. Brinkmann V, Reichard U, Goosmann C, Fauler B, Uhlemann Y, Weiss DS, Weinrauch Y, Zychlinsky A. Neutrophil extracellular traps kill bacteria. Science 303, 1532-1535 (2004).

44. Clark SR, Ma AC, Tavener SA, McDonald B, Goodarzi Z, Kelly MM, Patel KD, Chakrabarti S, McAvoy E, Sinclair GD, keys EM, Allen-Vercoe E, Devinney R, Doig CJ, Green FH, Kubes P. Platelet TLR4 activates neutrophil extracellular traps to ensnare bacteria in septic blood. Nat Med 13, 463-469 (2007).

45. Calderwood DA. Integrin activation. J Cell Sci 117, 657-666 (2004).

46. Shattil SJ, Newman PJ. Integrins: dynamic scaffolds for adhesion and signaling in platelets. Blood 104, 1606-1615 (2004).

47. Gachet C. P2 receptors, platelet function and pharmacological implications. Thromb Haemost 99, 466472 (2008).

48. Cosemans JM, Munnix IC, Wetzker R, Heller R, Jackson SP, Heemskerk JW. Continuous signaling via PI3K isoforms $\beta$ and $\gamma$ is required for platelet ADP receptor function in dynamic thrombus stabilization. Blood 108, 3045-3052 (2006).

49. Goto S, Tamura N, Ishida H, Ruggeri ZM. Dependence of platelet thrombus stability on sustained glycoprotein IIb/IIIa activation through adenosine $5^{\prime}$-diphosphate receptor stimulation and cyclic calcium signaling. J Am Coll Cardiol 47, 155-162 (2006).

50. Angelillo-Scherrer A, Burnier L, Flores N, Savi P, DeMol M, Schaeffer P, Herbert JM, lemke G, Goff SP, Matsushima GK, Earp HS, Vesin C, Hoylaerts MF, Plaisance S, Collen D, Conway EM, Wehrle-Haller B, Carmeliet P. Role of Gas6 receptors in platelet signaling during thrombus stabilization and implications for antithrombotic therapy. J Clin Invest 115, 237-246 (2005).

51. Bergmeier W, Stefanini L. Novel molecules in calcium signaling in platelets. J Thromb Haemost $\mathbf{7}$ Suppl 1, 187-190 (2009).

52. Honda A, Nogami M, Yokozeki T, Yanazaki M, Nakamura H, Watanabe H, kawamoto K, Nakayama K, Morris AJ, Frohman MA, Kanaho Y. Phosphatidylinositol 4-phosphate 5-kinase a is a downstream effector of the small G protein ARF6 in membrane ruffle formation. Cell 99, 521-532 (1999).

53. Martel V, Racaud-Sultan C, dupe S, Marie C, Paulhe F, Galmiche A, Block MR, Albiges-Rizo C. Conformation, localization, and integrin binding of talin depend on its interaction with phosphoinositides. J Biol Chem 276, 21217-21227 (2001).

54. Elvers M, Stegner D, Hagedorn I, Kleinschnitz C, braun A, Kuijpers ME, Boel M, Chen Q, Heemskerk JW, Stoll G, Frohman MA, Nieswandt B. Impaired $\mathrm{a}_{\mathrm{IIb}} \beta_{3}$ integrin activation and shear-dependent thrombus formation in mice lacking phospholipase D1. Sci Signal 3, ra1 (2010).

55. Romaniuk MA, Romaniuk MA, Croci DO, Lapponi MJ, Tribulatti MV, Negrotto S, Poirier F, Campetella O, Rabinovich GA, Schattner M . Binding of galectin-1 to aIIbb3 integrin triggers outside-in signals, stimulates platelet activation, and controls primary hemostasis. Faseb J 26, 2788-2798 (2012).

56. Brass LF, Stalker TJ, Zhu L, Lu B, Woulfe DS, Prevost N. Boundary events: contact-dependent and contact-facilitated signaling between platelets. Sem Thromb Hemost 30, 399-410 (2004).

57. Goodenough DA, Paul DL. Beyond the gap: functions of unpaired connexon channels. Nat Rev Mol Cell 
Biol 4, 285-294 (2003).

58. Goodenough DA, Paul DL. Gap junctions. Cold Spring Harbor Perspec Biol 1, 25-76 (2009).

59. Vaiyapuri S, Jones, CI, Sasikumar P, Moraes LA, Munger SJ, Wright JR, Ali MS, Sage T, Kaiser WJ, Tucker KL, Stain CJ, Bye AP, Jones S, Oviedo-Orta E, Simon AM, Mahaut-Smith MP, Gibbins. Gap junctions and connexin hemichannels underpin hemostasis and thrombosis. Circulation 125, 2479-2491 (2012).

60. Vaiyapuri S, Moreas LA, Sage T, ali MS, Lewis KR, Mahaut-Smith MP, Oviedo-Orta E, Simon AM, Gibbins JM. Connexin40 regulates platelet function. Nat Commun 4, 2564 (2013).

61. Naik MU, Stalker TJ, Brass LF, Naik UP. JAM-A protects from thrombosis by suppressing integrin $\mathrm{a}_{\mathrm{Itb}} \beta_{3}$-dependent outside-in signaling in platelets. Blood 119, 3352-3360 (2012).

62. Ozaki H, Ishii K, Arai $\mathrm{H}$, Horiuchi $\mathrm{H}$, Kawamoto T, Suzuki H, Kita. Junctional adhesion molecule (JAM) is phosphorylated by protein kinase C upon platelet activation. Biochem Biophys Res Commun 276, 873-878 (2000).

63. Kornecki E, Walkowiak B, Naik UP, Ehrlich YH. Activation of human platelets by a stimulatory monoclonal antibody. J Biol Chem 265, 10042-10048 (1990).

64. Naik UP, Ehrlich YH, Kornecki E. Mechanisms of platelet activation by a stimulatory antibody: crosslinking of a novel platelet receptor for monoclonal antibody F11 with the FcgRII receptor. Biochem J 310, 155-162 (1995).

65. Obergfell A, Eto K, Mocsai A, Buensuceso C, Moores SL, Brugge JS, Lowell CA, Shattil SJ. Coordinate interactions of Csk, Src, and Syk kinases with aIIbb3 initiate integrin signaling to the cytoskeleton. J Cell Biol 157, 265-275 (2002).

66. Jackson DE, Ward CM, Wang R, Newman PJ. The protein-tyrosine phosphatase SHP-2 binds platelet/ endothelial cell adhesion molecule-1 (PECAM-1) and forms a distinct signaling complex during platelet aggregation. J Biol Chem 272, 6986-6993 (1997).

67. Stalker TJ, Wu J, Morgans A, Traxler EA, Wang L, Chatterjee MS, Lee D, Quertermous T, Hall RA, Hammer DA, Diamond SL, Brass LF. Endothelial cell specific adhesion molecule (ESAM) localizes to platelet-platelet contacts and regulates thrombus formation in vivo. J Thromb Haemost 7, 1886-1896 (2009).

68. Tomlinson MG. Platelet tetraspanins: small but interesting. J Thromb Haemost 7, 2070-2073 (2009).

69. Stipp CS, Kolesnikova TV, Hemler ME. Functional domains in tetraspanin proteins. Trends Biochem Sci 28, 106-112 (2003).

70. Goschnick MW, Lau LM, Wee JL, Liu YS, Hogarth PM, Robb LM, Hickey MJ, Wright MD, Jackson DE. Impaired "outside-in" integrin $\mathrm{a}_{\mathrm{IIb}} \beta_{3}$ signaling and thrombus stability in TSSC6-deficient mice. Blood 108, 1911-1918 (2006).

71. Lau LM, Wee JL, Wright MD, Moseley GW, Hogarth PM, Ashman LK, Jackson DE. The tetraspanin superfamily member CD151 regulates outside-in integrin $\mathrm{a}_{\mathrm{IIb}} \beta_{3}$ signaling and platelet function. Blood 104, 2368-2375 (2004).

72. Dhanjal TS, et al. Minimal regulation of platelet activity by PECAM-1. Platelets 18, 56-67 (2007).

73. Patil S, Newman DK, Newman PJ. Platelet endothelial cell adhesion molecule-1 serves as an inhibitory receptor that modulates platelet responses to collagen. Blood 97, 1727-1732 (2001).

74. Rathore V, Stapleton MA, Hillery CA, Montgomery RR, Nichols TC, Merricks EP, Newman DK, Newman PJ. PECAM-1 negatively regulates GPIb/V/IX signaling in murine platelets. Blood 102, 3658-3664 (2003).

75. Martin V, Guillermet-Guibert J, Chicanne G, Cabou C, Jandrot-Perrus M, Plantavid M, Vanhaesebroeck B, Payrastre B, Gratacap MP. Deletion of the p110b isoform of phosphoinositide 3-kinase in platelets reveals its central role in Akt activation and thrombus formation in vitro and in vivo. Blood 115, 20082013 (2010).

76. Schoenwaelder SM, Ono A, Nesbitt WS, Lim J, Jarman K, Jackson SP. Phosphoinositide 3-kinase p110b regulates integrin $\mathrm{a}_{\mathrm{IIb}} \beta_{3}$ avidity and the cellular transmission of contractile forces. J Biol Chem 285, 2886-2896 (2010).

77. Ono A, Westein E, Hsiao S, Nesbitt WS, Hamilton JR, Schoenwaelder SM, Jackson SP. Identification of a fibrin-independent platelet contractile mechanism regulating primary hemostasis and thrombus growth. Blood 112, 90-99 (2008).

78. Auger JM, Watson SP. Dynamic tyrosine kinase-regulated signaling and actin polymerisation mediate aggregate stability under shear. Arterioscler Thromb Vasc Biol 28, 1499-1504 (2008).

79. Pleines I, Hagedorn I, Gupta S, May F, Chakarova L, Van Hengel J, Offermanns S, Krohne G, Klein schnitz C, Brakebusch C, Nieswandt B. Megakaryocyte-specific RhoA deficiency causes macrothrombocytopeniaand defective plateletactivation in hemostasisandthrombosis. Blood119, 1054-1063(2012). 
80. Buitrago L, Tsygankov A, Sanjay A, Kunapuli SP. Cbl proteins in platelet activation. Platelets 24, 419427 (2013).

81. Shcherbina A, Cooley J, Lutskiy MI, Benarafa C, Gilbert GE, Remold-O'Donnell E. WASP plays a novel role in regulating platelet responses dependent on $\mathrm{a}_{\mathrm{IIb}} \beta_{3}$ integrin outside-in signalling. $\mathrm{Br} \mathrm{J}$ Haematol 148, 416-427 (2010).

82. Kuijpers MJ, Schulte V, Oury C, Lindhout T, Broers J, Hoylaerts MF, Nieswandt B, Heemskerk. Facilitating roles of murine platelet glycoprotein Ib and $\mathrm{a}_{\mathrm{Ib}} \beta_{3}$ in phosphatidylserine exposure during VWF-collagen-induced thrombus formation. J Physiol 558, 403-415 (2004).

83. Kuijpers MJ, Schulte V, Bergmeier W, Lindhout T, Brakebusch C, Offermans S, Fässler R, Heemskerk JW. Complementary roles of glycoprotein VI and a2b1 integrin in collagen-induced thrombus formation in flowing whole blood ex vivo. Faseb J 17, 685-687 (2003).

84. Nieswandt B, Brakebusch C, Bergmeier W, Schulte V, Bouvard D, Mokharti-Nejad R, Lindhout $T$, Heemskerk JW, Zirngibl $H$, Fässler R. Glycoprotein VI but not $a_{2} \beta_{1}$ integrin is essential for platelet interaction with collagen. Embo J 20, 2120-2130 (2001).

85. Auger JM, Kuijpers MJ, Senis YA, Watson SP, Heemskerk JW. Adhesion of human and mouse platelets to collagen under shear: a unifying model. Faseb J 19, 825-827 (2005).

86. Consonni A, Cipolla L, Guidetti G, Canobbio I, Ciraolo E, Falasca M, Okigaki M, Balduini C, Torti M. Role and regulation of phosphatidylinositol 3-kinase $b$ in platelet integrin $a_{2} \beta_{1}$ signaling. Blood 119, 847-856 (2012).

87. Gilio K, Harper MT, Cosemans JM, Konopatskaya O, Munnix IC, Prinzen L, Leitges M, Liu Q, Molkentin JD, Heemskerk JW, Poole AW. Functional divergence of platelet protein kinase C (PKC) isoforms in thrombus formation on collagen. J Biol Chem 285, 23410-23419 (2010).

88. Konopatskaya O, Gilio K, Harper MT, Zhao Y, Cosemans JM, Karim ZA, Whiteheart SW, Molketin JD, Verkade P, Watson SP, Heemskerk JW, Poole AW. PKCa regulates platelet granule secretion and thrombus formation in mice. J Clin Invest 119, 399-407 (2009).

89. Konopatskaya O, Matthews SA, Harper MT, Gilio K, Cosemans JM, Williams CM, Navarro MN, Carter DA, Heemskerk JW, Leitges M, Cantrell D, Poole AW. Protein kinase C mediates platelet secretion and thrombus formation through protein kinase D2. Blood 118, 416-424 (2011).

90. Elvers M, Pozaj R, Pleines I, May F, Kuijpers MJ, Heemskerk JM, Yu P, Nieswandt B. Platelet hyperreactivity and a prothrombotic phenotype in mice with a gain-of-function mutation in phospholipase Cy2. J Thromb Haemost 8, 1353-1363 (2010).

91. Lecut C, Schoolmeester A, Kuijpers MJ, Broers JL, Van Zandvoort MA, Vanhoorelbeke K, Deckmyn $H$, Jandrot-Perrus $M$, Heemskerk JW. Principal role of glycoprotein VI in $a_{2} \beta_{1}$ and $a_{\mathrm{II}} \beta_{3}$ activation during collagen-induced thrombus formation. Arterioscler Thromb Vasc Biol 24, 1727-1733 (2004).

92. Munnix IC, Gilio K, Siljander PR, Raynal N, Feijge MA, Hackeng TM, Deckmyn H, Smethurst PA, Farndale RW, Heemskerk JW. Collagen-mimetic peptides mediate flow-dependent thrombus formation by high- or low-affinity binding of integrin $a_{2} \beta_{1}$ and glycoprotein VI. J Thromb Haemost 6, 2132-2142 (2008).

93. Siljander PR, Munnix IC, Smethurst PA, Deckmyn H, Lindhout T, Ouwehand WH, Farndale RW, Heemskerk. Platelet receptor interplay regulates collagen-induced thrombus formation in flowing human blood. Blood 103, 1333-1341 (2004).

94. Van de Walle GR, Schoolmeester A, Iserbyt BF, Cosemans JM, Heemskerk JW, Hoyaerts MF, Nurden $A$, Vanhoorelbeke $K$, Deckmyn H. Activation of $a_{\text {IIt }} \beta_{3}$ is a sufficient but also an imperative prerequisite for activation of $a_{2} \beta_{1}$ on platelets. Blood 109, 595-602 (2007).

95. Hodivala-Dilke KM, McHugh KP, Tsakiris DA, Rayburn H, Crowley D, Ullman-Culleré, Ross FP, Coller BS, Teitelbaum S, Hynes RO. $\beta 3$-integrin-deficient mice are a model for Glanzmann thrombasthenia showing placental defects and reduced survival. J Clin Invest 103, 229-238 (1999).

96. Yang H, Kim A, David T, Palmer D, Jin T, Tien J, Huang F, Cheng T, Coughlin SR, Jan YN, Jan LY. TMEM16F forms a $\mathrm{Ca}^{2+}$-activated cation channel required for lipid scrambling in platelets during blood coagulation. Cell 151, 111-122 (2012).

97. Randriamboavonjy V, Isaak J, Frömel T, Viollet B, Fisslthaler B, Preissner KT, Fleming I. AMPKa2 subunit is involved in platelet signaling, clot retraction, and thrombus stability. Blood 116, 2134-2140 (2010).

98. Chen X, Zhang Y, Wang Y, Li D, Zhang L, Wang K, Luo X, Yang Z, Wu Y, Liu J. PDK1 regulates platelet activation and arterial thrombosis. Blood 121, 3718-3726 (2013).

99. Gushiken FC, Hyojeng H, Pradhan S, Langlois KW, Alrehani N, Cruz MA, Rumbau RE, Vjayan V. The catalytic subunit of protein phosphatase $1 \gamma$ regulates thrombin-induced murine platelet $\mathrm{a}_{\mathrm{ILb}} \beta_{3}$ function. Plos One 4, e8304 (2009). 
100. Kuchay SM, Kim N, Grunz EA, Fay WP, Chishti AH. Double knockouts reveal that protein tyrosine phosphatase $1 B$ is a physiological target of calpain-1 in platelets. Mol Cell Biol 27, 6038-6052 (2007).

101. Arias-Salgado EG, Haj F, Dubois C, Mran B, Kasirer-Friede A, Furie BC, Neel BG, Shattil SJ. PTP-1B is an essential positive regulator of platelet integrin signaling. J Cell Biol 170, 837-845 (2005).

102. Stefanini $L$, Boulaftali $Y$, Ouellette TD, Holinstat M, Désiré $L$, Leblond $B$, Andre $P$, Coney PB, Bergmeier W. Rap1-Rac1 circuits potentiate platelet activation. Arterioscler Thromb Vasc Biol 32, 434-441 (2012).

103. Zhang G, et al. Distinct roles for Rap1b protein in platelet secretion and integrin $\mathrm{a}_{\mathrm{IIb}} \beta_{3}$ outside-in signaling. J Biol Chem 286, 39466-39477 (2011).

104. Haling JR, Monkley SJ, Critchley DR, Petrich BG. Talin-dependent integrin activation is required for fibrin clot retraction by platelets. Blood 117, 1719-1722 (2011).

105. Buitrago L, Langdon WY, Sanjay A, Kunapuli SP. Tyrosine phosphorylated c-Cbl regulates platelet functional responses mediated by outside-in signaling. Blood 118, 5631-5640 (2011).

106. Takizawa H, Nishimura S, Takayama N, Oda A, Nishikii H, Morita Y, Kakinuma S, Yamazaki S, Okamura S, Tamura N, Goto S, Sawaguchi, Manabe I, Takatsu K, Nakauchi H, Takaki S, Eto K. Lnk regulates integrin $\mathrm{a}_{\mathrm{IIb}} \beta_{3}$ outside-in signaling in mouse platelets, leading to stabilization of thrombus development in vivo. J Clin Invest 120, 179-190 (2010).

107. Weyrich AS, Denis MM, Schwertz H, Tolley ND, Foulks J, Spencer E, Kraiss LW, Albertine KH, McIntyre TM, Zimmerman GA. mTOR-dependent synthesis of Bcl-3 controls the retraction of fibrin clots by activated human platelets. Blood 109, 1975-1983 (2007).

108. Spinelli SL, Casey AE, Polock SJ, Gertz JM, McMillan DH, Narasipura SD, Mody NA, King R, Maggirwar SB, Francis CW, Taubman MB, Blumberg N, Phipps RP. Platelets and megakaryocytes contain functional nuclear factor-kappaB. Arterioscler Thromb Vasc Biol 30, 591-598 (2010).

109. Zhao $Y$, Zhang $Y$, Wang $S$, Hua Z, Zhang J. The clock gene Per2 is required for normal platelet formation and function. Thromb Res 127, 122-130 (2011).

110. Feijge MA, Ansink K, Vanschoonbeek K, Heemskerk JW. Control of platelet activation by cyclic AMP turnover and cyclic nucleotide phosphodiesterase type-3. Biochem Pharmacol 67, 1559-1567 (2004).

111. Suzuki-Inoue $K$, Hughes $C E$, Inoue $O$, Kaneko M, Cuyun-Lira O, Takafuta $T$, Watson SP, Ozaki Y. Involvement of Src kinases and PLCY2 in clot retraction. Thromb Res 120, 251-258 (2007).

112. Severin S, Gratacap MP, Lenain N, Averez L, Hollande E, Penninger JM, Gachet C, Plantavid M, Payrastre B. Deficiency of Src homology 2 domain-containing inositol 5-phosphatase 1 affects platelet responses and thrombus growth. J Clin Invest 117, 944-952 (2007).

113. Lecut C, Feijge MA, Cosemans JM, Jandrot-Perrus M, Heemskerk JW. Fibrillar type I collagens enhance platelet-dependent thrombin generation via glycoprotein VI with direct support of $a_{2} \beta_{1}$ but not $a_{\text {IIb }} \beta_{3}$ integrin. Thromb Haemost 94, 107-114 (2005).

114. Vanschoonbeek K, Feijge MA, Van Kampen RJ, Kenis H, Hemker HC, Giesen PL, Heemskerk JW. Initiating and potentiating role of platelets in tissue factor-induced thrombin generation in the presence of plasma: subject-dependent variation in thrombogram characteristics. J Thromb Haemost 2, 476-484 (2004).

115. Gong H, Shen B, Flevaris P, Chow C, Lam SC, Voyno-Yasenetskaya TA, Kozasa T, Du X. G protein subunit Ga13 binds to integrin $\mathrm{a}_{\mathrm{IIb}} \beta_{3}$ and mediates integrin "outside-in" signaling. Science 327, 340-343 (2010).

116. Kannan M, Ahmed RP, Jain P, Kumar R, Choudhry VP, Saxena R. Type I Glanzmann thrombasthenia: most common subtypes in North Indians. Am J Hematol 74, 139-141 (2003).

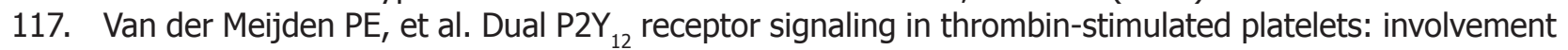
of phosphoinositide 3-kinase $\beta$ but not $\gamma$ isoform in $\mathrm{Ca}^{2+}$ mobilization and procoagulant activity. Febs J 275, 371-385 (2008).

118. Van der Meijden PE, Feijge MA, Swieringa F, Gilio K, Nergiz-Unal R, Hamulyák K, Heemskerk JW. Key role of integrin $\mathrm{a}_{\mathrm{IIb}} \beta_{3}$ signaling to Syk kinase in tissue factor-induced thrombin generation. Cel Mol Life Sci 69, 3481-3492 (2012). 



\section{CHAPTER 8}

GENES IN ARTERIAL THROMBUS FORMATION AND BLEEDING:

TRANSLATION OF META-DATA FROM MOUSE STUDIES

De Witt SM, Meacham S, Feijge MA, Baaten CC, Jupe S, Turro E, Wiese A, Akkerman JW, Ouwehand WH, Prins MH, Cosemans JM, Heemskerk JW 


\section{Summary}

Current antiplatelet and anticoagulant therapies effectively, albeit partly, reduce cardiovascular disease by preventing arterial thrombosis and thromboembolism. However, this ameliorating effect is at the expense of increased risk of bleeding. Hence, continued search is needed to find new targets for antithrombotics without side effects. Studies in which mice with genetic modification or mice treated pharmacology are subjected to experimental in vivo arterial thrombosis, thromboembolism and/or in vitro thrombus formation provide unique insight into the roles of hundreds of proteins in the thrombotic process. We performed an extensive PubMed search over the years 1980-2013 to identify such studies. Subsequently, a scoring procedure to quantitatively compare the effect of genetic modification or pharmacological inhibition on time-dependent, mass-dependent and stability dependent parameters of in vivo arterial thrombosis and in vitro thrombus formation, experimental thromboembolism, and tail bleeding time was developed. A meta-analysis of the scores for 1080 published mouse studies, representing 315 genes, resulted in 44 genes/proteins controlling arterial thrombus formation without affecting hemostasis. Class analysis indicated a strong correlation between collagen-dependent in vivo and in vitro models of thrombus formation. Transfer of the current scores per mouse gene (protein) to human databases indicated that a substantial part of the Reactome pathways in the hemostasis field were covered. Analysis using human networks further revealed that several of the identified 44 genes were on highly connected nodes, suggesting that these may provide effective targets for antithrombotic therapy. In summary, this work demonstrates that a meta-analysis integrating small size mouse studies: (i) provides a contribution to 3R alternative approach for in vivo experimentation, (ii) establishes a high degree of homology of murine and human thrombotic processes, and (iii) identifies new molecular targets for antithrombotic therapy.

\section{Introduction}

Cardiovascular disease, resulting from arterial thrombosis or thromboembolism, is still a leading cause of major disability and death worldwide 1 . Research during the last decades has resulted in successful therapeutic means to reduce cardiovascular disease manifestations, e.g. by drugs lowering plasma lipids and blood vessel wall tension. In particular, antiplatelet and anticoagulant drugs - directed to a dozen of molecular targets - are used for the secondary prevention of thrombotic events and infarctions ${ }^{2,3}$. However, while these treatments are effective in thrombosis reduction, they have an increased risk of minor or major bleeding as common side effect ${ }^{4}$. Hence, there is continued interest to search for new targets of drugs that are thromboprotective without causing or aggravating hemorrhages. For the identification of such targets, experimental thrombosis models using genetically modified mice have appeared to be invaluable $3,5,6$.

Up to now, hundreds of mouse studies have been performed to evaluate the molecular mechanisms of arterial thrombus formation or thromboembolism in vivo. The majority of this work concerns general or tissue-specific genetic modifications or pharmacological interventions, resulting in an altered platelet or coagulant function or a modification of endothelial processes. Small size reviews, published so far, provide qualitative descriptions of the changes in thrombus formation in genetically modified mice $3,6,7$. This qualitative approach however makes it not easy to compare studies using different methodologies. With respect to mouse models of bleeding risk, a review of Wei et al. describes the effects of genetic modification on tail bleeding time in relation to changed platelet properties ${ }^{8}$. Importantly, widely used in vivo mouse models of arterial thrombosis and bleeding appear to be sensitive to the majority of antithrombotic drugs with established therapeutic efficacy in man. Accordingly, these models may help to identify novel genes that contribute to thrombus formation with minimal bleeding risk 9,10 .

Flow chamber devices operating with isolated blood are increasingly being employed as a proxy outcome for in vivo thrombus formation, for instance to assess the effect of gene modification on thrombus formation in vitro ${ }^{11,12,13}$. These in vitro methods not only can serve as alterna- 
tives for in vivo animal experiments (for each of the 3Rs: refinement, reduction and replacement), but also are relevant from a translational point of view to investigate effects of new pharmacological agents. Although there is a need for further standardization for use with human blood ${ }^{14}$, flow chamber assays have been shown to detect genetic or acquired deficiencies in platelet function and to sense the efficacy of antithrombotic medication $15,16,17,18$. In addition, they provide increased knowledge into signaling processes implicated in hemostasis and thrombosis ${ }^{19}, 20$.

In general, published studies on arterial thrombosis or thromboembolism in mice have a common experimental approach, in that vascular damage is induced by either activation or denudation of the endothelium, with in the latter case exposure of the collagen-containing subendothelial matrix. However, experimental procedures of the studies can greatly differ in: the type vascular bed used, the type and strength of trigger to disturb the endothelium or provoke thromboembolism, the equipment and detection method employed and the parameters used for thrombosis quantification. The experimental outcome is furthermore known to be influenced by the high complicity of the interventions and the experimental skills of the operating technician ${ }^{10}$.

Considering that repetition of published mouse studies is not desirable for ethical reasons, here we hypothesized that analysis of meta-data obtained through systematic quantitative comparison of published studies provides additional insight into the cooperation of genes contributing to arterial thrombus formation in vivo and in vitro. Furthermore, by comparing the mouse gene networks with those recently obtained from human gene sequence variation data, we supposed that the murine meta-data provide novel insight into genes regulating human thrombosis and bleeding. To this end, we developed and validated a scoring procedure to quantitatively compare published studies on murine arterial thrombus formation, thromboembolism and tail bleeding. This scoring method provided novel, comparative insight into the roles of 315 mouse genes in experimental thrombosis with or without altered bleeding, and allowed identification of new molecular targets for treatment of human cardiovascular disease.

\section{Methods}

\section{Selection of papers and definition of studies}

A PubMed search over the years 1980-2013 was performed to identify papers on mouse studies and arterial thrombus formation. Keywords were mouse $\&$ arterial \& thrombus, or mouse $\&$ thromboembolism in different combinations. We searched for primary papers: (i) in English language; (ii) describing effects of modification of a specific mouse gene or protein on arterial thrombosis formation or thromboembolism; and (iii) providing quantitative data with statistics. Excluded were papers not indicating effects of a well-defined genetic or pharmacological perturbation, on venous thrombosis models only or containing only qualitative data. A second PubMed search was performed to identify papers describing quantitative effects of genetic modification on tail bleeding times (keywords: mouse, tail bleeding, tail clipping, knockout \& gene in different combinations). Non-tail bleeding times were not included. All papers of the described sets as well as published reviews were further screened for additional primary papers.

Individual studies were identified from the included papers. A study was defined as the quantitative measurement of thrombus formation or thromboembolism in vivo or in vitro under a specific set of conditions (vascular bed, injury trigger, detection method) for a perturbed mouse group (genetically modified or pharmacologically inhibited) in comparison to the unperturbed group (corresponding wildtype or pharmacological control), such as defined by the authors. Papers describing multiple models to measure thrombus formation, e.g. in different vessels or in vivo and in vitro, hence, provided more than one study.

Registry of perturbed genes, experimental variables and outcome data per study An encompassing registry was made of all studies by categorizing these per gene or protein cor- 
responding to that gene. Full data of this registry are given in Suppl. Table 1, also providing all primary references (online available only). Basal genetic data were taken from standard databases (http://www.informatics.jax.org): gene symbols and names; mouse genome index; chromosome location; Uniprot identification number of the corresponding human protein. Listed for each study were the: genetic background; type of genetic modification; pharmacological intervention if applicable; mouse phenotype regarding altered activity of platelets, coagulation, fibrinolysis or vessel wall.

Recorded experimental details on the prothrombotic intervention differed per study class (Suppl. Table 1). Listed for class I (arterial thrombosis in vivo) were the vessel and type of injury and the dosing with the duration of injury. Recorded for class II (systemic thromboembolism in vivo) were: type of intervention (unprovoked or provoked by injection of collagen/epinephrine, tissue factor) and the dosing of intervention. Listed for class III (thrombus formation in vitro) were: the type of flow device, thrombogenic surface and blood flow conditions (shear rate, presence of anticoagulation). With respect to tail bleeding (class IV), only the bleeding time was listed.

Each study was searched for quantitative information regarding the thrombotic or thromboembolic process for the perturbed (genetic modification or pharmacological intervention) and unperturbed (wildtype or control) condition. Also recorded were experimental variables (type of measurement, fluorescence, Doppler flow, surface-area coverage, etc.). Differences in mean or median values between mice with(out) perturbation were listed as statistically significant, if indicated so in the original publication.

\section{Grouping of studies and outcome parameters}

Studies were grouped according to major similarities in intervention protocols (Table 1). Groups 1 to 12 (class I) were defined by the vascular bed and the way of thrombosis induction. The severity (dosing or time) of intervention was not used for grouping, as this is often incompletely described and is subjected to investigator-dependent operation procedures ${ }^{10}$. Groups $20-23$ (class II) were formed based on the way of (provoked) systemic intervention or the presence of unprovoked thromboembolism. Groups 30-34 (class III) were formed, depending on the type of thrombogenic surface during in vitro blood flow. Studies of class IV were not sub-clustered.

The quantitative data were listed per class (Table 1). For in vivo and in vitro thrombus formation (classes I and III), measurements were categorized as time-dependent parameters (a), mass-dependent parameters (bi: extent of platelet adhesion; bii: extent of occlusion or surface-area-coverage; biii: number, size or volume of thrombi), or stability parameters (c, number or frequency of embolic events). For in vivo thromboembolism (class II), data were categorized as provoked (d, percentage of survival, time to death, residual platelet count) or unprovoked (e, spontaneous thrombosis or bleeding).

\section{Comparing all data by systematic scoring}

Per study and parameter, listed quantitative data were scored at a 3-point and 5-point scale (Figure 1). On the 3-point scale, measurements pointing to a significant reduction or delay in thrombus formation (or thromboembolism) after perturbation (genetic modification or pharmacological intervention) were scored as -1 ; unchanged effects as 0 ; and measurements indicating a significant increase or acceleration of thrombus formation as +1 . On the 5 -point scale, a further distinction was made between data designating a reduced/delayed thrombus formation with $<50 \%$ (score -2 ) or $\geq 50 \%$ (score -1 ); and between data indicating an increased/accelerated thrombus formation with $\leq 200 \%$ (score +1$)$ or $>200 \%$ (score +2$)$. Tail bleeding times were scored at 3-point scale only: prolonged $(-1)$, unchanged $(0)$, shortened $(+1)$. The original published values and scored parameters are given in Suppl. Table 1. Averaged scores per study, gene, group and class were rounded off on 3-point or 5-point scale. 
Overrepresentation analysis in Reactome

Overrepresentation analysis of orthologous human proteins, corresponding to the analyzed mouse genes, was performed using the Analyze Data tool in the Reactome pathway database ${ }^{21}$.

Chromosome mapping and network analysis

Chromosome mapping was done with the Circos program ${ }^{22}$. Networks were constructed in Cytoscape to explore effects of other human proteins (genes) on arterial thrombus formation and bleeding. Networks were constructed using the Reactome pathways as a core set to find potential genes that regulate human thrombosis and bleeding; edges were extended by using protein-protein interactions from the IntAct database to connect all nodes. Pre-established data from human GWAS and Thrombogenomics analyses and transcription profiles in human megakaryocytes were implemented in the network constructions as well (S. Meacham \& W. Ouwehand, unpublished).

Table 1. Cataloging of analyzed mouse studies. Indicated are assignments of studies to classes and groups, as well as listed output parameters of thrombus formation, thromboembolism or tail bleeding.

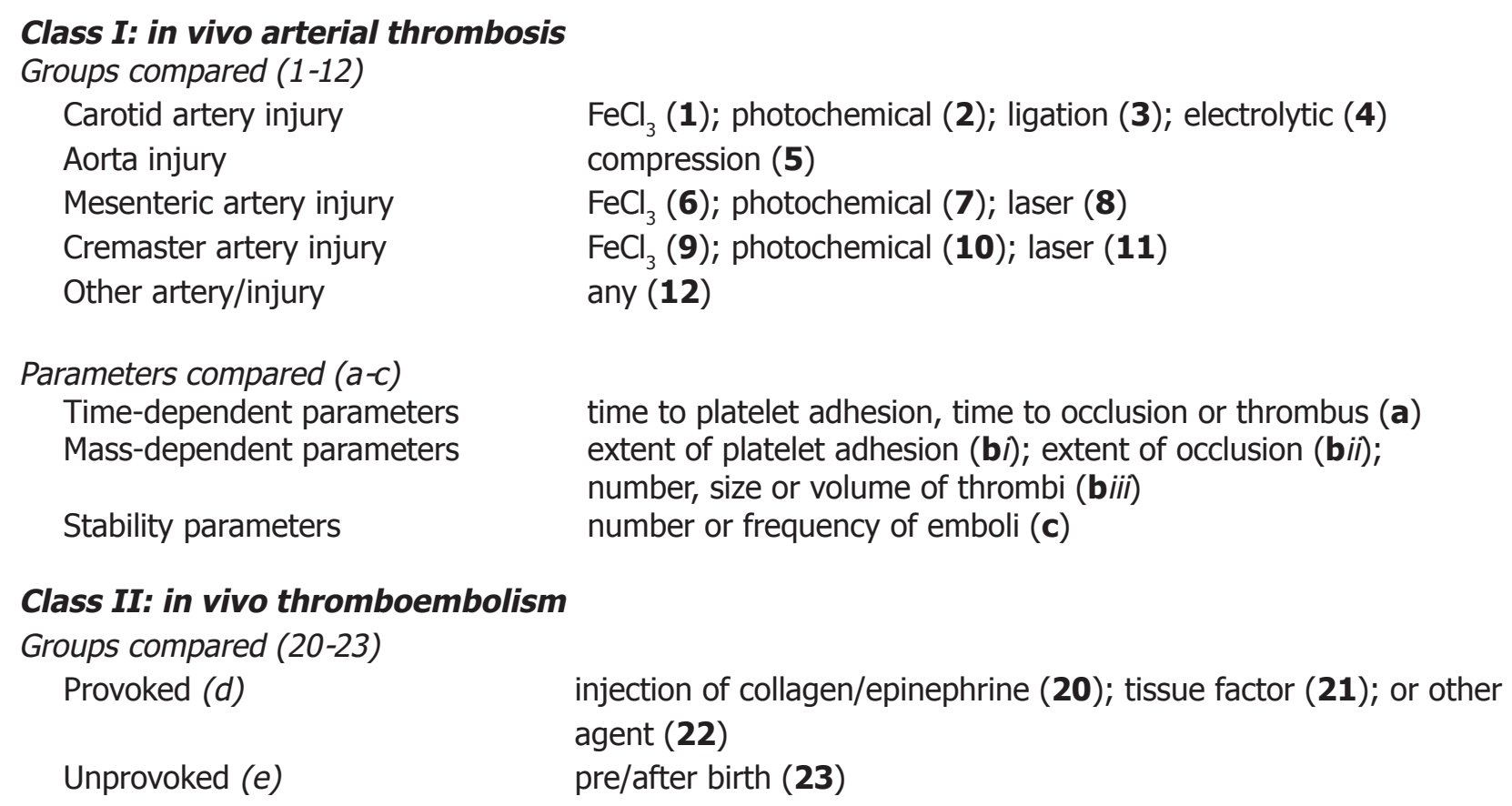

Parameters compared

Thromboembolism parameters

percentage death, time to death, residual platelet count (d); unprovoked thrombosis or bleeding (e)

\section{Class III: in vitro thrombus formation}

Groups compared (30-34)

Adhesive surface

Parameters compared $(f-h)$

Time-dependent parameters

Mass-dependent parameters

Stability parameters

Class IV: bleeding time collagen I (30); collagen III (31); VWF (32), fibrinogen (33); other (34)

time to platelet adhesion, time to thrombus formation (f) extent of platelet adhesion ( $\mathbf{g} i)$; surface area coverage ( $\mathbf{g} i$ ); number, size or volume of thrombi ( $\mathbf{g}$ iii) number or frequency of emboli (h)

tail bleeding time 


\section{Statistics and correlation of parameters}

Statistics on the effects of gene perturbation on thrombus formation in individual studies were taken from the original papers. Correlation analysis was performed to compare, per study and class, scores of time-dependent, mass-dependent and thrombus stability parameters. The non-parametric Kendall's Tau-c test was used to determine association or rank correlation. Herein, the Kendall's $\tau$ value is computed as the excess of tabled concordant over discordant pairs, divided by a term representing the geometric mean of untied pairs. It reaches from 0 to 1.0 for tables where all entries are on one diagonal. The statistical package for the social sciences was used for all analyses (SPSS version 22, IBM Statistics).

\section{Results and Discussion}

Included mouse studies on arterial thrombosis, thromboembolism and tail bleeding

A PubMed search for scientific papers on arterial thrombus formation or thromboembolism in mouse over the period 1980-2013 resulted in 1244 publications. Another PubMed search for papers on mouse tail bleeding resulted in 626 publications. The majority of these papers did not describe effects that could be related to a specific molecular target or did not contain quantitative information, and hence were not used. In total, 431 papers contained numeral data on effects of a that could be linked to a single gene or protein perturbation, and assessed experimental arterial thrombosis in vivo (class I), systemic thromboembolism in vivo (class II), thrombus formation at arterial shear rate in vitro (class III), or tail bleeding (class IV). These papers as well as published reviews were screened for further primary sources, giving another 35 publications. Individual studies taken from these papers were defined as interventions using a particular perturbation and a particular vascular bed or method. The result was a large database with information on 1080 different studies, described in 466 publications. For classes I, II, III and IV, this concerned 435, 83, 182 and 380 studies, respectively (see meta-data in Suppl. Table 1).

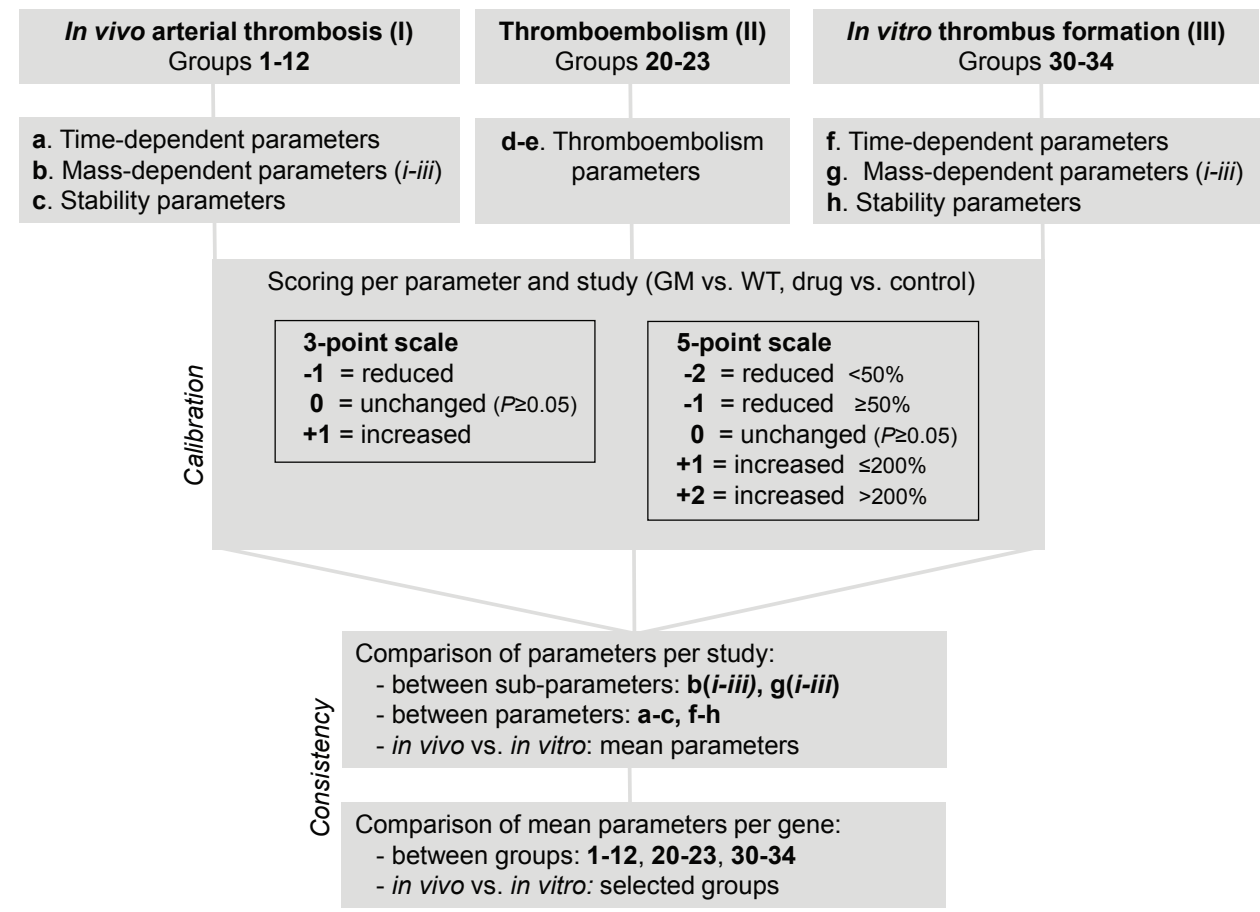

Figure 1. Flow scheme used for scoring, calibration and consistency analysis of parameters of in vivo arterial thrombus formation (class I), thromboembolism (class II), or in vitro thrombus formation (class III) in mice with genetic modification (GM) or treated pharmacologically, in comparison to wildtype (WT) or control condition. Indicated are scoring rules of output parameters at 3- and 5-point scales (values for wildtype normalized to 100\%). For full description of groups, see Table 1. 
Scoring of parameters of thrombus formation, thromboembolism and tail bleeding per study Since different methods have been employed in the field to provoke and assess thrombus formation in mice (Table 1), we developed a procedure allowing quantitative comparison of the outcome of published studies independently of the method used. Therefore, we scored predefined parameters of arterial thrombus formation in vivo (class I) or in vitro (class III) on both a 3-point and 5-point scale (Figure 1). The rationale for this is that such scoring indicates a significant decrease or increase (3-point); or alternatively, a moderate/strong decrease or moderate/strong increase (5-point) of relevant parameters determining the thrombotic process at the perturbed condition, i.e. genetic modification or pharmacological intervention. Correlation analysis, applied to the database as a whole, indicated significant correlations of three distinguishable mass-dependent sub-parameters within individual studies of class I or class III, both at 3-point and 5-point scale, with Kendall's $\tau \geq 0.60$ ( $P \leq 0.029$ ) (Suppl. Table 2A-B). This indicated that subscores can be combined into one mass-dependent score per study. Furthermore, correlation analysis pointed to an overall high similarity of time-dependent, mass-dependent and stability parameters, again within studies (Suppl. Table 2C-D). In class I, these parameters correlated significantly with $P<$ 0.001 , except when comparing the time and stability parameters at 5 -point scale $(P=0.063)$. In class III, with lower sample size, the time- and mass-dependent parameters correlated with $P=$ 0.046 (3-point) or 0.074 (5-point); the stability parameter was hardly in use. Because of this overall similarity of intra-study scores, we decided to combine these into one 'calibrated' outcome score per study (3- or 5-point scale).

Studies to arterial thromboembolism (class II) were categorized as provoked or unprovoked, and scored in a single category (Figure 2). Since tail bleeding times (class IV) were often reported to be prolonged for an undefined time, these were scored on a 3-point scale only.

A

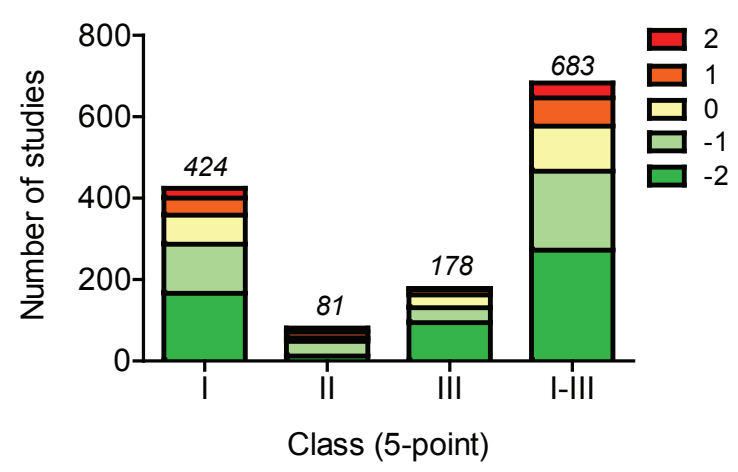

B

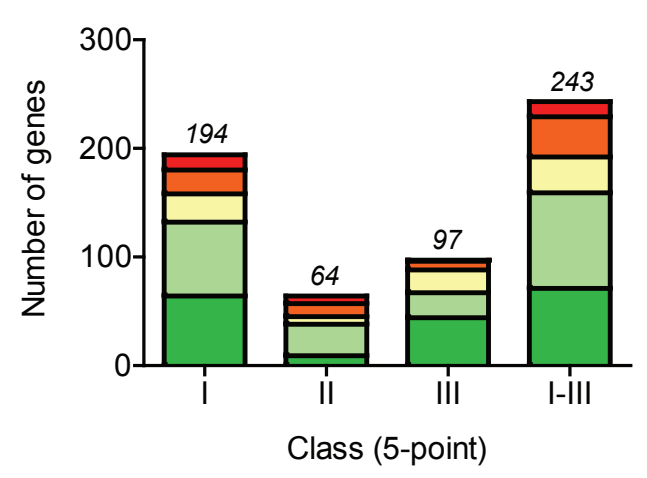

C

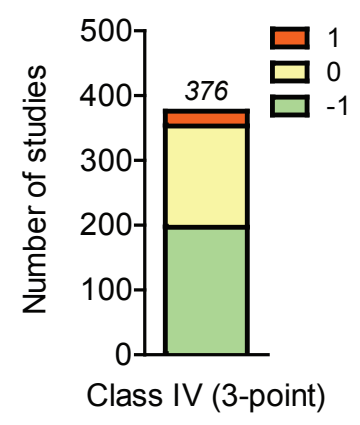

D

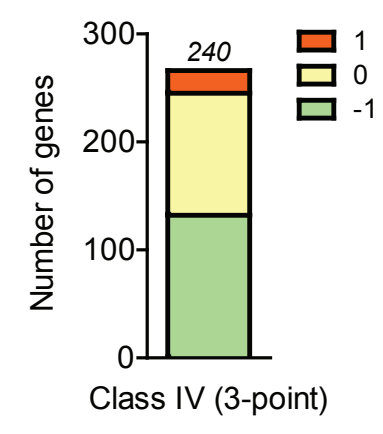

Figure 2. Score distribution of analyzed studies (A, C) and genes (B, D) for classes I-III and IV, as indicated. Scoring was at 3-point scale (A, B) or 5-point scale (C, D). Represented are numbers of studies and genes per class for mice with a loss-of-gene-function. Note that studies using mice with non-comparable genetics (for example, gain-of-function mutations) are not included. 


\section{Consistency of scores per gene within classes and experimental groups}

The large database size allowed analysis of the consistency of the combined scores per study. Since a large number of papers included class I or II in vivo studies together with class III in vitro studies using the same breeding of animals, we compared the scores in these classes per paper. This gave for both class I $(P<0.001, n=87)$ and class II $(P \leq 0.012, n=12)$ significant correlations at 3- and 5-point scales (Suppl. Table 3A). Hence, for the database as a whole and within papers, the study scores for in vitro thrombus formation corresponded well to these of in vivo arterial thrombosis and thromboembolism.

To compare the scores for different thrombosis models, studies were grouped according to key experimental variables, in particular the type of artery and the method to provoke vascular injury (Table 1). In class I, the mostly used vascular beds were carotid artery $>$ mesentery $>$ cremaster > aorta; while the most popular injury method declined with $\mathrm{FeCl}_{3}>$ laser $>$ photochemical reagent $>$ vessel ligation or compression > electrolytic (Suppl. Figure 1A-B). In class II, the majority of studies used injection of collagen/epinephrine, with fewer studies using tissue factor or other injection or reporting unprovoked thrombosis (Suppl. Figure 1C). Concerning class III, the majority of studies used collagen-I as thrombogenic surface (Suppl. Figure 1D). Class IV studies were not sub-divided.

Murine thrombosis studies are usually carried out at small sample sizes $(n=6-12)$. Hence, in comparing groups and classes, we averaged the scores from different papers with mice with equivalent genetic modifications (or pharmacological interventions) without weighting. The matrix of average scores per group indicated that numbers were mostly too low for statistical comparison of individual groups (Suppl. Table 4). Therefore, we performed this analysis for combinations of groups, clustered per type of vessel or type of injury. Markedly, scores (averaged per gene) of studies using the carotis, mesentery or cremaster correlated significantly at 3-point and 5-point scales (Table 2). With respect to injury type, scores for $\mathrm{FeCl}_{3}$ injury correlated significantly with those for laser or photochemical injury only at 3-point scale. This significance was lost at 5-point scale, which is compatible with the evidence that the determinants of collagen-dependent (i.e. $\mathrm{FeCl}_{3}$ or ligation/compression) and collagen-independent (i.e. laser or photochemical) thrombosis models are partly different 22,23 .

To take this further, we compared the scores (averaged per gene) of collagen-(in)dependent in vivo studies of classes I-II with those of class III studies, i.e. thrombus formation on collagen in vitro. As indicated in Table 3, this resulted in highly significant correlations for studies using the carotis or mesentery, and in less significant correlations for cremaster studies (with frequent laser injury, Suppl. Figure 2). Per injury type, correlations were again stronger for studies using $\mathrm{FeCl}_{3}$ or ligation/compression injury (collagen-dependent) than for studies with laser or photochemical injury. For thromboembolism (class II), scores for studies using collagen/epinephrine injection correlated well with those of collagen-dependent thrombus formation in vitro $(P \leq 0.032)$.

\section{Comparing roles of genes in arterial thrombus formation, thromboembolism and tail bleeding}

Given the overall agreement of scores per gene, when comparing groups and classes, we concluded that, for the database as a whole, the effect of gene modification is larger than the variation caused by differences in methodology or measurement parameters. This is a marked result, indicating overall consistency between the various in vivo and in vitro thrombosis models, regarding determination of an antithrombotic or prothrombotic phenotype. The 5-point scores typically differed from the 3-point scores in that the former were more influenced by the known collagen-dependency of the thrombosis model. Since the 5-point (class) scores are more discriminative in comparing the roles of different genes in arterial thrombus formation or thromboembolism, these were used for further analyses.

By combining scores from studies per gene and class, quantitative information was obtained on the process of thrombus formation or thromboembolism for 194, 64 and 97 genes in classes I, 
Table 2. Correlation of study scores between in vivo thrombus formation (class I) with different vascular beds and different triggers (indicated groups). Kendall's Tau-test for 3- and 5-point scoring scales. Indicated are groups compared and numbers $(N)$ of genes compared. For description of groups, see Table 1.

\begin{tabular}{|c|c|c|c|c|c|c|}
\hline & \multicolumn{2}{|c|}{ Groups $N$} & \multicolumn{2}{|c|}{ 3-Point scale } & \multicolumn{2}{|c|}{ 5-Point scale } \\
\hline & & & Kendall's T & $P$ & Kendall's T & $P$ \\
\hline Compared to carotis & $(1-4)$ & & & & & \\
\hline Mesentery & $6-8$ & 39 & 0.86 & $<0.001$ & 0.57 & $<0.001$ \\
\hline Cremaster & $9-11$ & 20 & 0.82 & 0.019 & 0.55 & 0.010 \\
\hline Compared to $\mathrm{FeCl}_{3}$ & $(1,6,9)$ & & & & & \\
\hline Laser & 8,11 & 27 & 0.75 & $<0.001$ & 0.17 & 0.293 \\
\hline Photochemical & $2,7,10$ & 18 & 0.44 & 0.048 & 0.36 & 0.062 \\
\hline Ligation \& compression & 3,5 & 17 & 0.38 & n.d.* & 0.40 & 0.050 \\
\hline
\end{tabular}

*Not determined (constant variable).

Table 3. Correlation of scores for in vivo thrombus formation (indicated groups) with in vitro thrombus formation on collagen (group 30). Kendall's Tau-test for 3- and 5-point scoring scales. $N$, number of genes compared. For description of groups, see Table 1 .

\begin{tabular}{|c|c|c|c|c|c|c|}
\hline \multirow[t]{2}{*}{ Compared to group $\mathbf{3 0}$} & \multicolumn{2}{|c|}{ Groups $N$} & \multicolumn{2}{|c|}{ 3-Point scale } & \multicolumn{2}{|c|}{ 5-Point scale } \\
\hline & & & Kendall's T & $P$ & Kendall's T & $P$ \\
\hline Class I all & $(1-12)$ & 71 & 0.66 & $<0.001$ & 0.58 & $<0.001$ \\
\hline Carotis & $1-4$ & 46 & 0.63 & $<0.001$ & 0.49 & $<0.001$ \\
\hline Mesentery & $6-8$ & 34 & 0.80 & $<0.001$ & 0.63 & $<0.001$ \\
\hline Cremaster & $9-11$ & 22 & 0.51 & 0.020 & 0.34 & 0.076 \\
\hline $\mathrm{FeCl}_{3}$ & $1,6,9$ & 53 & 0.57 & $<0.001$ & 0.53 & $<0.001$ \\
\hline Laser & 8,11 & 23 & 0.75 & $<0.001$ & 0.40 & 0.039 \\
\hline Photochemical & $2,7,10$ & 18 & n.d.* & n.d. & 0.21 & 0.519 \\
\hline Ligation \& compression & 3,5 & 10 & 0.68 & $<0.001$ & 0.56 & 0.010 \\
\hline Class II all & $(20-23)$ & 23 & 0.66 & 0.001 & 0.30 & 0.099 \\
\hline Collagen/epinephrine & 20 & 10 & 0.97 & $<0.001$ & 0.64 & 0.032 \\
\hline
\end{tabular}

*Not determined (constant variable). 

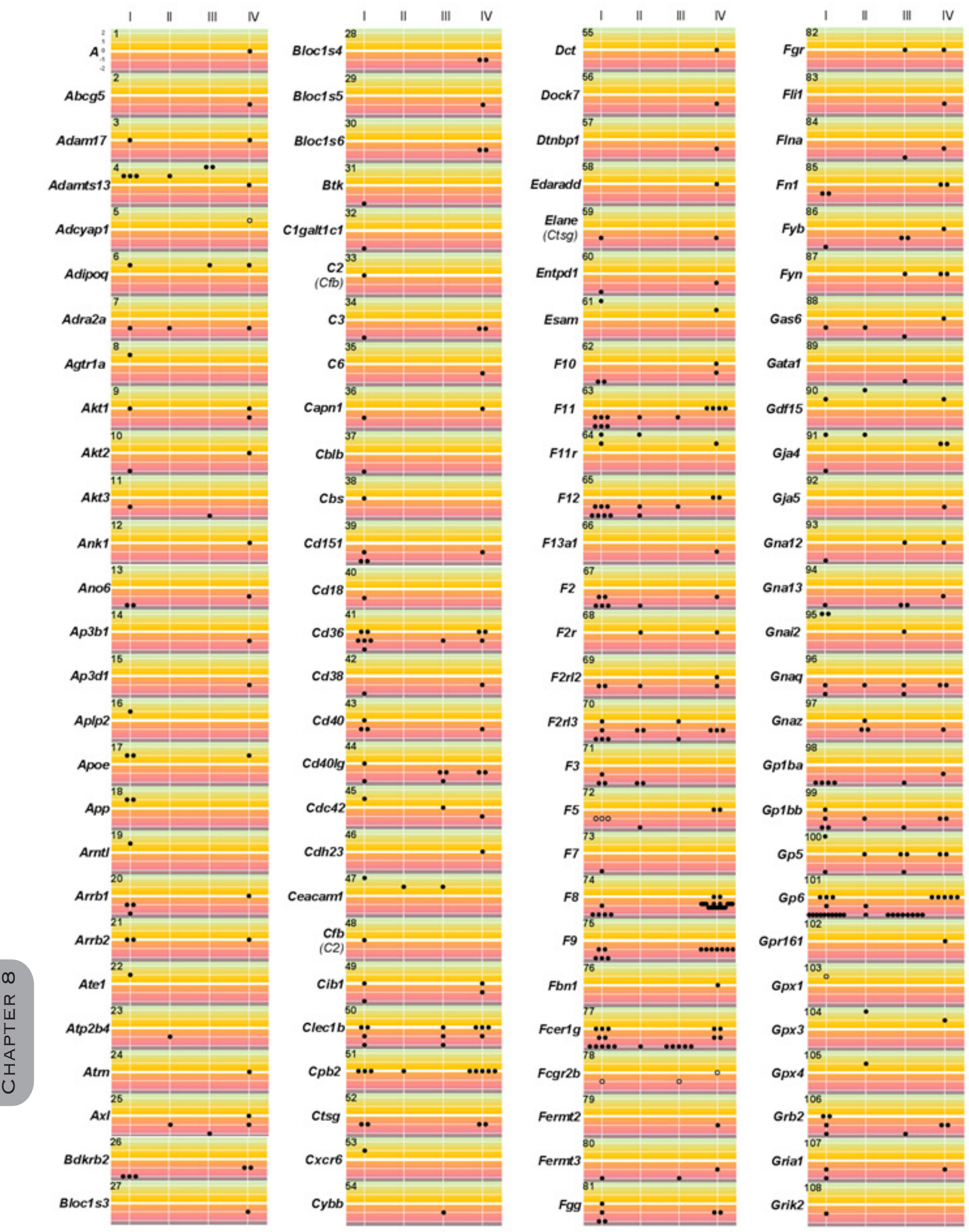

Figure 3. Forest Plot 

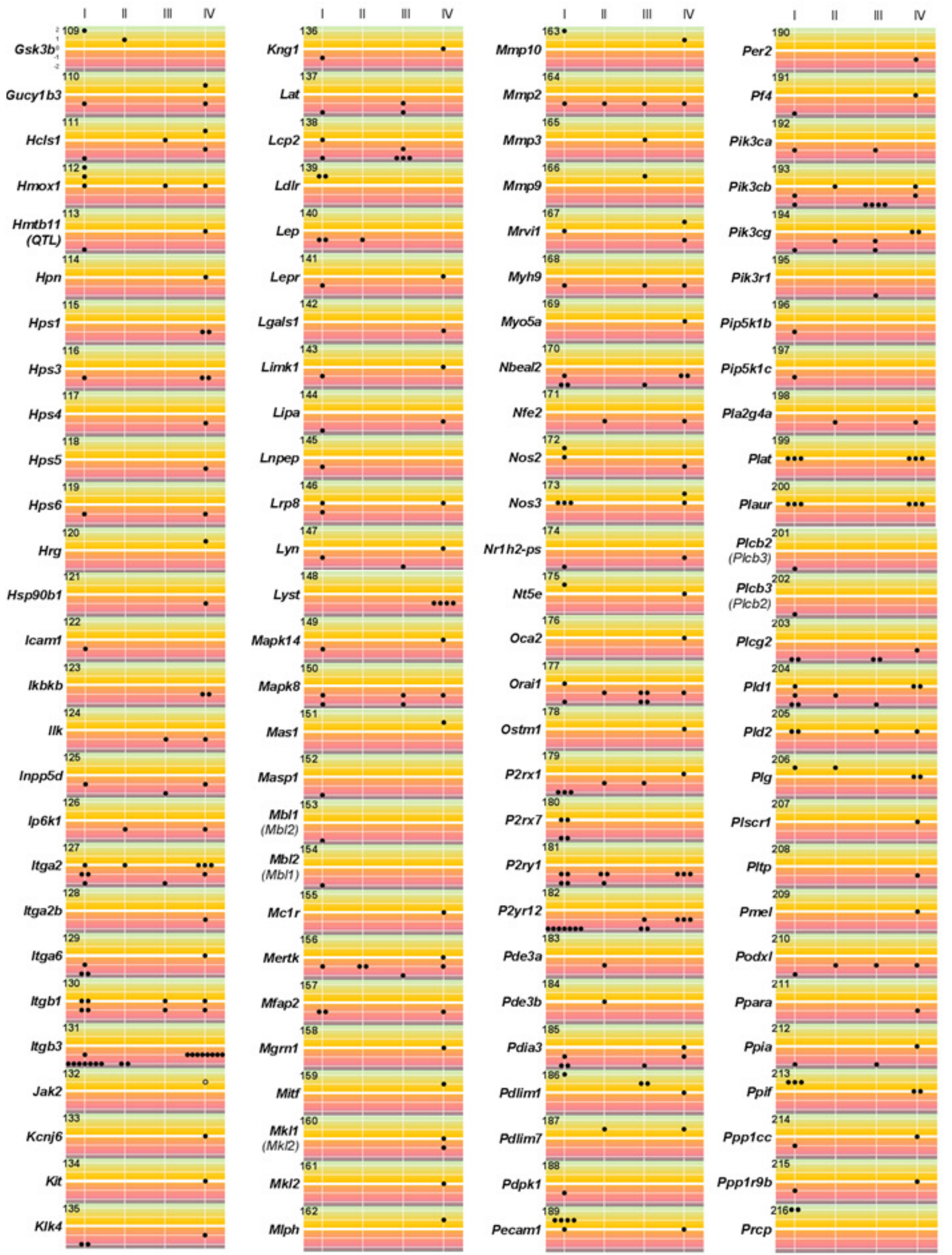

Figure 3. Forest Plot (continuous) 

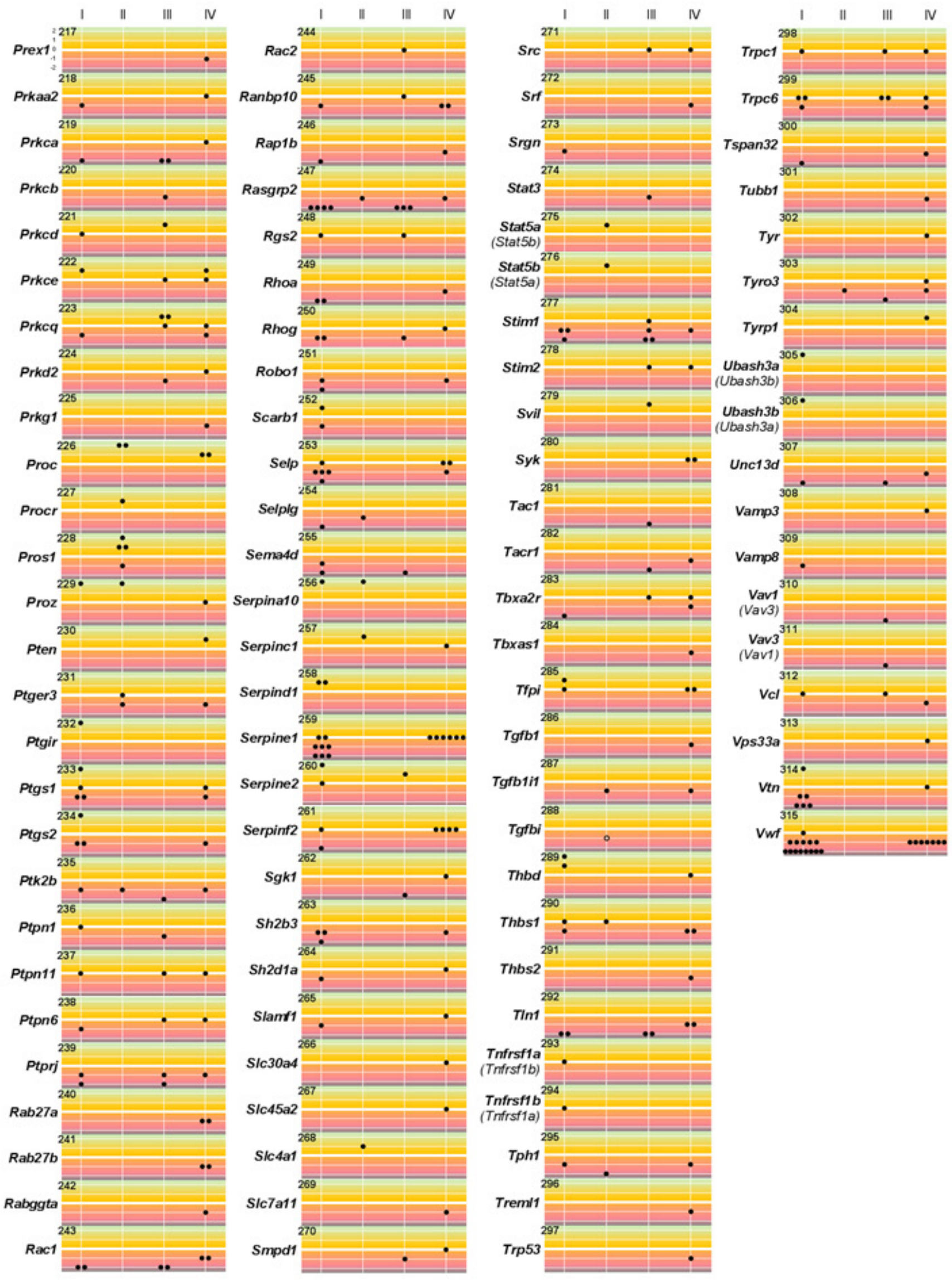

Figure 3. Forest Plot (continuous) 
Figure 3. Forest plot of scored studies on effects of gene modification or drug intervention on murine arterial thrombosis (classes I-III, 5-point) or tail bleeding (class IV, 3-point). Each bullet refers to a single published study. Mouse genes are ordered alphabetically according to current nomenclature. For full names, mean values and relevant phenotypic changes, see Suppl. Table 5. Open bullets refer to a gain-of-gene-function study, scored as if it concerned loss-of-function. Score legend: -2 , strongly reduced thrombosis; -1 , reduced thrombosis or prolonged bleeding time; 0 , unchanged thrombosis or bleeding time; +1 , increased thrombosis or shortened bleeding time; +2 strongly increased thrombosis.

II and III, respectively (Figure 2B). Scores for bleeding times (class IV) were listed for 240 genes (Figure 2D). For all classes together, information was obtained on 315 genes (Suppl. Table 1). As indicated in Figure 3, showing an overview of all study scores for each of the 315 genes, in the majority of cases scores clustered towards an antithrombotic (scores $-2,-1)$, unchanged (score 0 ), or prothrombotic (scores $+1,+2$ ) phenotype, regardless of the study class. An extensive list with overall scores per gene of class I-III and class IV is given in Suppl. Table 5, also providing additional information on the effect of genetic modification on platelet and coagulant functions.

For 25 of the most investigated genes ( $n \geq 6$ studies, $86 \%$ collagen-dependent models), we analyzed the score distributions (Figure 4A). Acknowledging different effect sizes of these genes and assuming this independent of class and group, the chance of 'correct' final score was calculated as a function of the number of most frequently included studies (Figure 4B, C). This prediction analysis indicated for one study a median probability to obtain a correct answer of 0.83 (3-point) or 0.50 (5-point). For two studies of the same gene, the median probability was 0.98 (3-point) or 0.85 (5-point). Overall, for obtaining a $85 \%$ chance of correct answer, a single study is sufficient when scored at 3-point scale, and two studies appear to be required when scored at 5-point scale. Based on this analysis, use of the 5-point scale becomes most useful with data from two studies available.

Particularly interesting for the thrombosis field are those genetic modifications that lead to reduced arterial thrombosis (negative scores) without increased bleeding. In the current database, as many as 44 genes contribute to such a phenotype (Figure 5). On the other hand, gene modifications resulting in shortened bleeding times were in 11 out of 12 cases accompanied by a prothrombotic tendency (positive scores). Further analysis indicated that a -2 score was more frequently (79\%) accompanied by bleeding prolongation than a -1 score $(51 \%)$. This underlines the hypothesis that a more severe reduction in arterial thrombus formation is more likely to result in a bleeding phenotype.

Chromosome mapping indicated even distribution of positive and negative scores on all chromosomes with the exception of chromosome 3, 5, 6, 15, 18 and X. Genes located on chromosome $3,5,6,15$ and $X$ were almost all exclusively associated with an antithrombotic tendency and thus an increased risk for bleeding, while genes located on chromosome 18 were associated with a prothrombotic phenotype. Genes located on chromosome $Y$ are involved in male development and are as such not involved in thrombosis and hemostasis. (Suppl. Figure 3).

\section{Translation to human cardiovascular disease and bleeding}

For translation of the scored mouse data to the human situation, first an overrepresentation analysis was performed using the Reactome database, which is a curated knowledgebase of biological pathways, including human megakaryocytes and platelets ${ }^{24}$. Comparing all proteins, orthologous to the corresponding human genes, this analysis indicated that a substantial and significant part of the hemostasis pathway was covered (Figure 6). Well-covered sub-pathways included the gamma-carboxylation of coagulation factors, G-protein coupled receptors and signaling, as well as several protein tyrosine phosphorylation and phospholipase sub-pathways. Human Reactome analysis of proteins corresponding to mouse genes, scored for arterial thrombosis (class I-III) or bleeding (class IV), indicated that the majority of the above-mentioned pathways are over-represented in the thrombosis studies (Suppl. Figure 4). 
A

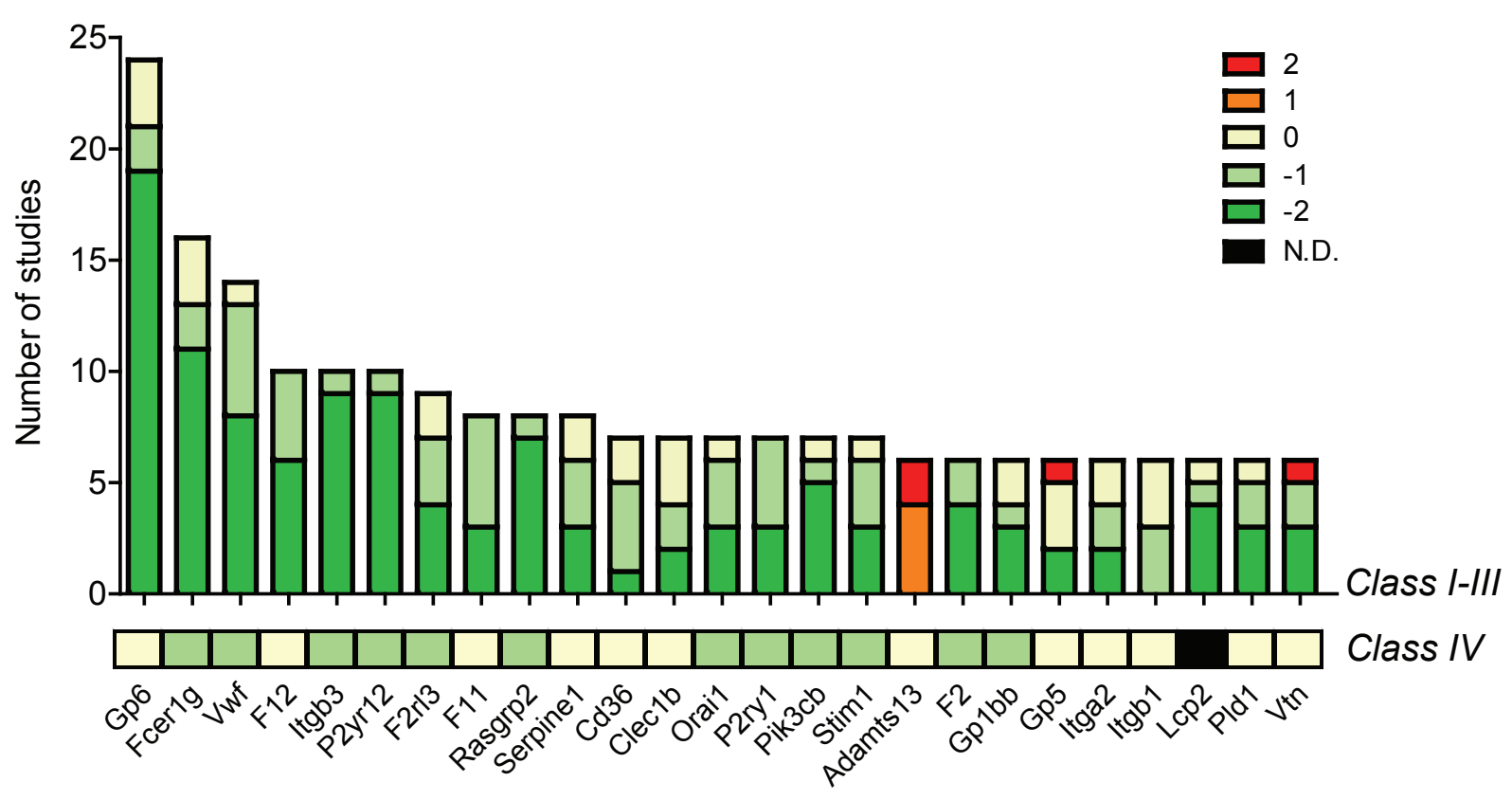

B

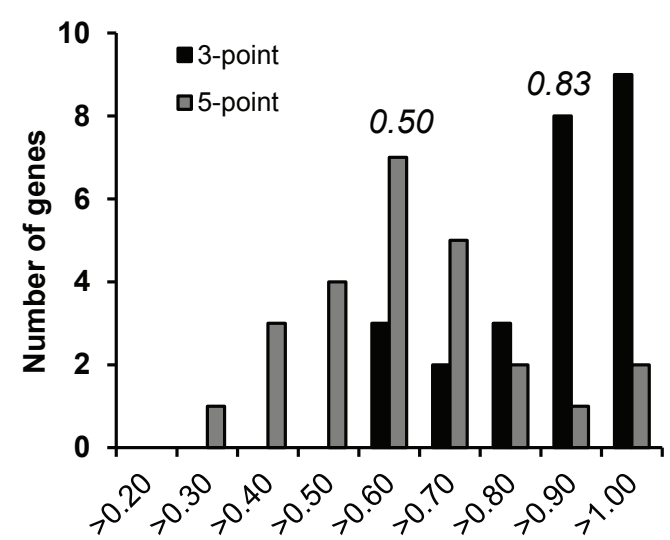

C

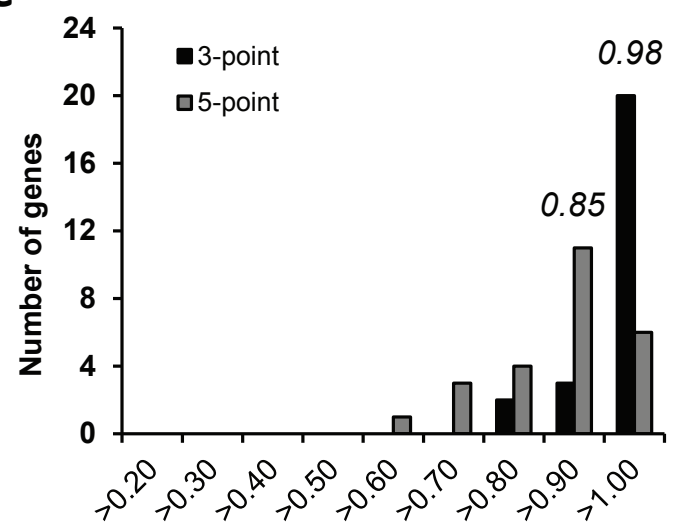

Figure 4. Ranking of 25 most frequently studied mouse genes for effects on arterial thrombosis tendency in vivo or in vitro. (A) Summative study scores per gene for classes I-III; also given are the mean bleeding scores (class IV). Data come from 216 studies (87\% collagen-dependent models; $9 \%$ laser models, 3\% photochemical injury). (B, C) Prediction of correct score, assuming that only one (B) or two (C) studies are known for each of the 25 genes (3-point or 5-point scale, as indicated). Given are histograms of probability deciles and median values.

The Reactome database was also used as core set for construction of a network of orthologous human genes (Suppl. Figure 5). Searching for nodes connected to at least to nodes with negative scores for thrombus formation resulted in 18 genes, of which 11 have been implicated in platelet and/or integrin activation or coagulation (BCAR1, CD2AP, CRK, FADK1, FGA, FGFR2, GGCX, IRS1, NPHS1, SPAG9, TEK).

A more extended network analysis, also using datasets from GWAS, Thrombogenomics analyses and transcription profiles in human megakaryocytes, was performed to compare the mouse scores for 44 genes (proteins) which contribute to thrombus formation without effect on bleeding time (Figure 7). Phenotyping evaluation indicated that the majority of the corresponding proteins are involved in platelet activation (receptors, signaling or granule release), while other proteins have a role in the coagulation process (Table 4). On the other hand, interestingly, genes that contribute to the halt of bleeding but not to thrombus formation in part have a regulatory role in the platelet actin cytoskeleton and the vascular endothelium (Table 4). 
Table 4. Orthologous human genes, translated from scores of mice with reduced arterial thrombosis without bleeding, or prolonged bleeding without reduced arterial thrombosis. Genes are classified according to altered processes in the genetically modified mice.

Process

\section{Human gene (orthologue)}

\section{Antithrombotic, no prolonged bleeding}

Coagulation (plasma)

F12, F11, KNG1, LEPR, SERPINE1, SERPINE2

Platelet aggregation (plasma)

FN1, GAS6, VTN

Platelet-granule released

PF4

Platelet receptor

CD36, CLEC1B, FCGR2B, GP6, ITGA2, ITGA6, ITGB1, LRP1, P2RX1, SELP

Platelet signaling AKT2, CAPN1, FYB, GNA12, HCLS1, LIMK1, LYN, MAPK14, PIK3CG, PLD1, PPIA, PRKCA, PPP1CC, PPP1R9B, PRKAA2, PRKD2, PTPN6, RHOG, SGK1, SH2D1A, SLAMF1, SMPD1

Unknown Hmtb11 (mouse QTL)

\section{Prolonged bleeding, not antithrombotic}

Endothelial activation

Platelet cytoskeleton

Platelet-granule released

Platelet signaling
NOS2, PTGS1, PTGS2

$C D C 42, V C L$

THBS1

AKT1
A

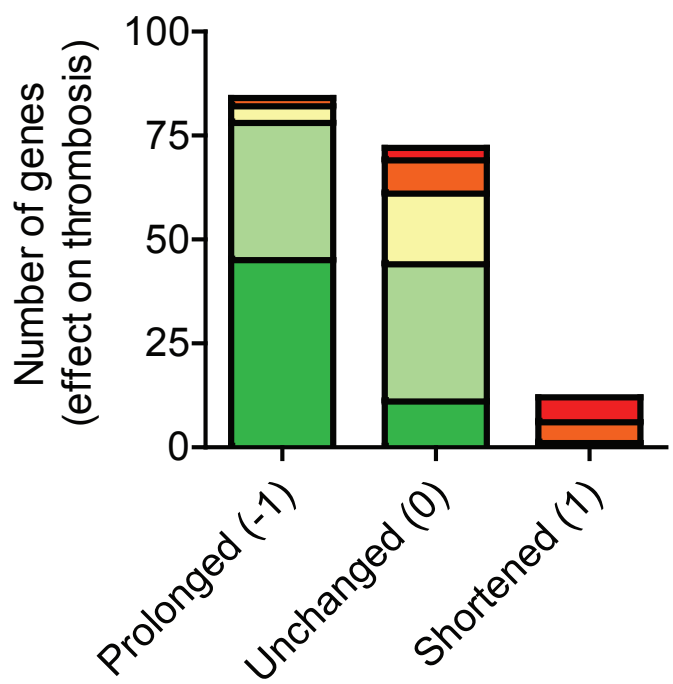

Tail bleeding (class IV)
B

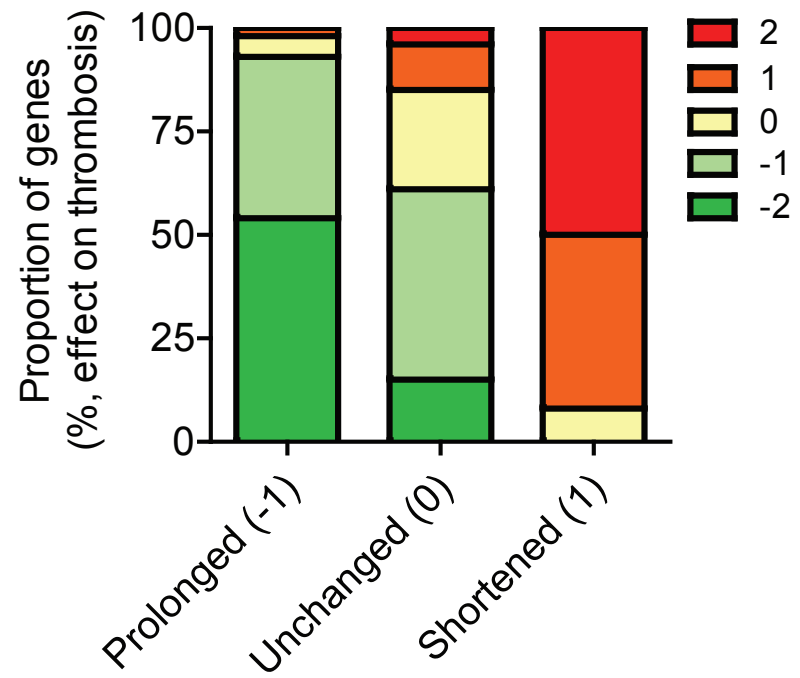

Tail bleeding (class IV)

Figure 5. Effects of gene deficiencies on arterial thrombus formation or thromboembolism (5-point scale, classes I-III), in combination with a prolonged $(-1)$, unchanged $(0)$ or shortened $(+1)$ tail bleeding time. Shown are numbers $(\mathbf{A})$ and proportions (B) of analyzed genes. 


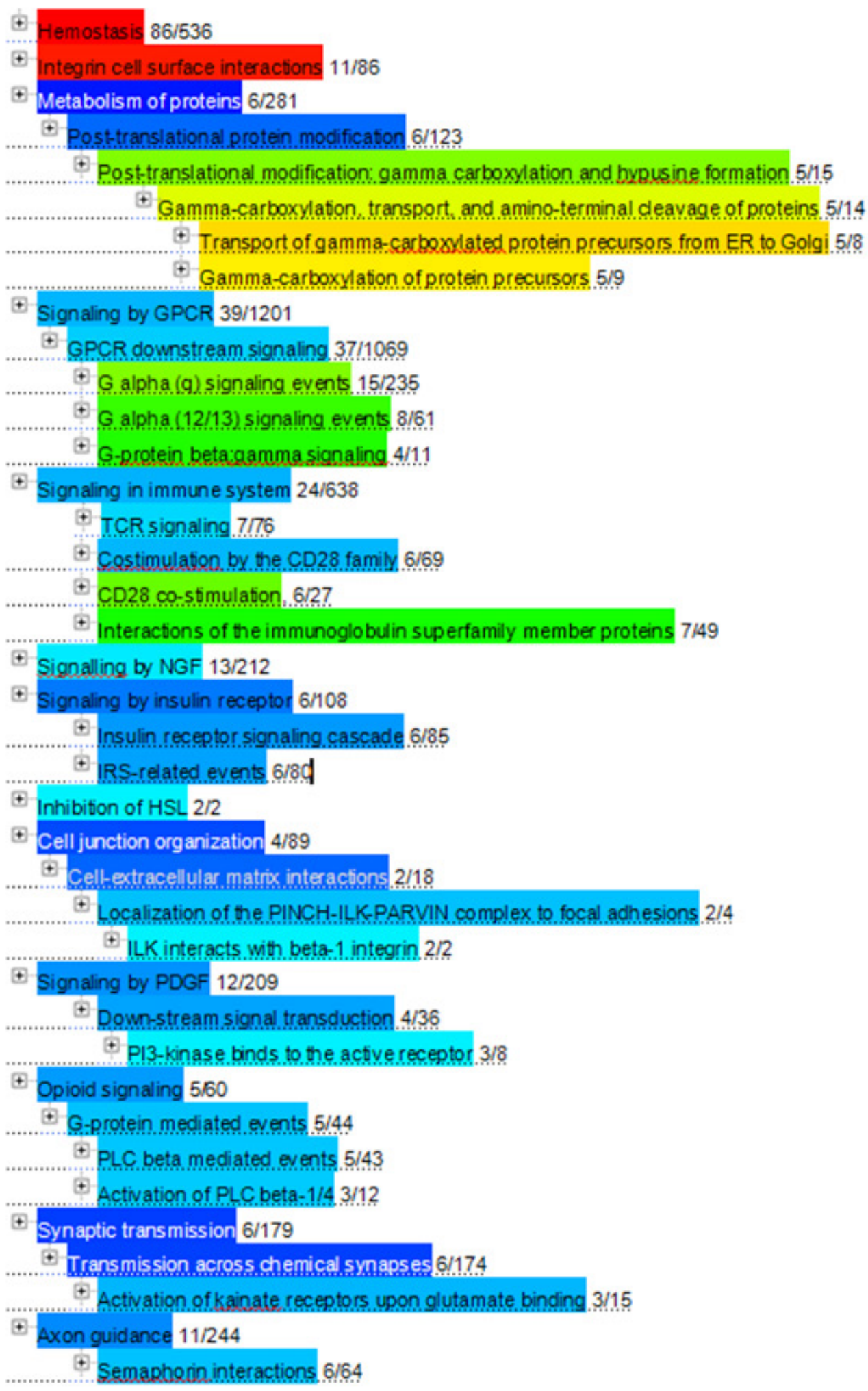

Figure 6. Reactome hierarchical over-representation analysis of orthologous human proteins corresponding to mouse genes with scores for arterial thrombus formation and/or bleeding. Numbers per pathway (Reactome event) are given. Each event is coloured according to the probability of seeing the given number or more proteins in this pathway by chance. The top-level (root) pathways are ordered according to the lowest p-value of their components. Total number of events assessed was 5187, number of matching events was 379, number of proteins (genes) matching submitted identifiers was 105. 
A

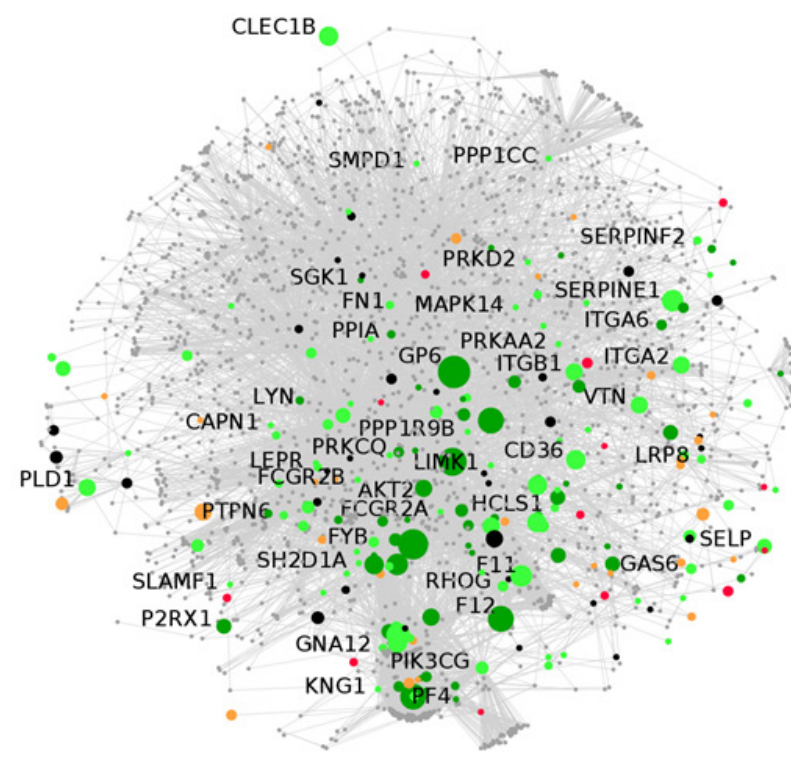

B $\bullet 2$

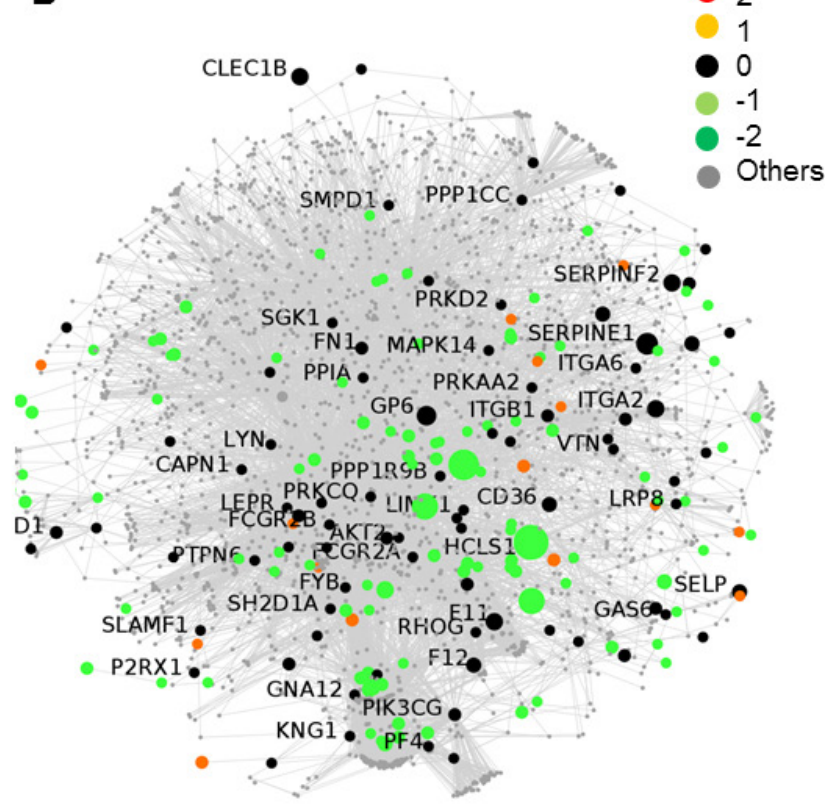

Figure 7. Visualization of mouse genes implicated in arterial thrombosis (A) or bleeding (B) using extended network of orthologous human genes. Human gene network was constructed copy from GWAS and Thrombogenomics analyses and transcription profiles in human megakaryocytes. Highlighted are positions of genes mentioned in Table 4. Color key of nodes for $A$ is on the 5-point scale, for B on the 3-point scale. Grey lines are the edges coming from IntAct (protein interactions). The size of the nodes is based on the amount of mouse studies performed per gene perturbation. Highlighted genes are 44 genes as indicated in table 4 .

\section{Conclusions}

Mouse experimental arterial thrombosis studies, performed in a translational research context, have in common several characteristic features, such as genetic homogeneity, small sample size, controlled experimental conditions, and real-time monitoring of the thrombus or thromboembolism. On the other hand, the mode and target vessel of injury as well as the recording of output parameters can differ greatly from laboratory to laboratory ${ }^{10}$. In the present paper, we show that uniform scoring of the effect of genetic modification on the thrombotic process is feasible and provides better insight in the suitability of different experimental models. Given the similarity in scorings, between models and classes, the present meta-analysis points to an overall comparable molecular regulation of the thrombus-forming process in different arterial beds, and even for systemic (collagen-induced) thromboembolism. On the other hand, the scoring on 5-point scale reveals particular differences between known collagen-dependent and -independent thrombosis models. The latter models (laser or photochemical injury) are more dependent on tissue factor with mild endothelial damage and less on collagen exposure, although this may depend on the severity of the trigger $22,23,25$. In human disease, collagen exposure due to atherosclerotic plaque rupture is considered to be an important factor in arterial thrombus formation ${ }^{26,27}$, but which of the current mouse models are best predictive for human thrombotic disorders is still unknown.

Given the currently limited molecular targets for treatment of cardiovascular disease, the present meta-analysis points to a wealth of novel candidate target proteins with a role in murine arterial thrombosis and/or hemostasis. Mostly, these are genes modulating functions of platelets, blood lipids, coagulation or fibrinolysis, but also genes regulating the vessel wall; it is to be expected that especially the latter list will grow in the near future. Systematic analysis elucidates 44 genes encoding for such proteins that markedly affect arterial thrombosis and/or thromboembolism in mouse without affecting hemostasis. 
The present analysis furthermore shows that, at least for collagen-dependent thrombosis models, ex vivo measurement of thrombus formation using flow chambers can be used as a proxy test for measurements in vivo. This finding may in several ways support $3 \mathrm{R}$ animal alternatives approaches to limit in vivo cardiovascular research by: (i) providing quantitative information on effects of new genetic modification and multiple gene deficiencies, in order to replace in vivo experiments; (ii) defining when small-volume ex vivo flow assays are valid alternatives for in vivo thrombosis measurement, thus reducing numbers of tested animals.

Transfer of the current scores per mouse gene (protein) to human databases indicated that a substantial part of the Reactome pathways in the hemostasis field were covered. Network analysis further revealed that several 'hotspots' where the (mouse) genes appeared on highly connected nodes, suggesting that these also play a role in human cardiovascular disease. In summary, this work demonstrates that a meta-analysis integrating small size mouse studies: (i) provides a contribution to $3 \mathrm{R}$ alternative approach for in vivo experimentation, (ii) establishes a high degree of homology of murine and human thrombotic processes, and (iii) identifies new molecular targets for antithrombotic therapy.

\section{Acknowledgements}

We thank Reyhan Nergiz-Unal for analytical support. This work was supported by grants from the Center for Translational Molecular Medicine (INCOAG), the Netherlands Heart Foundation (2011T6), the Landsteiner Foundation for Blood Transfusion Research (1006) and ZonMW (MKMD 114021004); the National Institute for Health Research (BRIDGE-BPD) and the Wellcome Trust, United Kingdom

\section{References}

1. World Health Organization. Factsheet Cardiovascular diseases Factsheet No: 317, http://www.who.int/ mediacentre/factsheets/fs317/en/ (2013).

2. Mackman N. Triggers, targets and treatments for thrombosis. Nature 451, 914-918 (2008).

3. Jackson SP. Arterial thrombosis: insidious, unpredictable and deadly. Nat Med 17, 1423-1436 (2011).

4. Swieringa F, Kuijpers MJ, Heemskerk JW, van der Meijden PE. Targeting platelet receptor function in thrombus formation: the risk of bleeding. Blood Rev 28, 9-21 (2014).

5. Nieswandt B, Pleines I, Bender M. Platelet adhesion and activation mechanisms in arterial thrombosis and ischaemic stroke. J Thromb Haemost 9 (Suppl. 1), 92-104 (2011).

6. Westrick RJ, Ginsburg D. Modifier genes for disorders of thrombosis and hemostasis. J Thromb Haemost 7 Suppl 1, 132-135 (2009).

7. Borissoff JI, Spronk HM, ten Cate $\mathrm{H}$. The hemostatic system as a modulator of atherosclerosis. $\mathrm{N}$ Engl J Med 364, 1746-1760 (2011).

8. Wei AH, Schoenwaelder SM, Andrews RK, Jackson SP. New insights into the haemostatic function of platelets. Br J Haematol 147, 415-430 (2009).

9. Nieswandt B, Varga-Szabo D, Elvers M. Integrins in platelet activation. J Thromb Haemost 7 Suppl 1, 206-209 (2009).

10. Denis CV, Dubois C, Brass LF, Heemskerk JW, Lenting PJ. Towards standardization of in vivo thrombosis studies in mice. J Thromb Haemost 9, 1641-1644 (2011).

11. Zwaginga JJ, Sakariassen KS, Nash G, King MR, Heemskerk JW, Frojmovic M, Hoylaerts MF. Flow based assays for global assessment of hemostasis. Part 2: current methods and considerations for the future. J Thromb Haemost 4, 2716-2717 (2006).

12. Emerson M. Refinement, reduction and replacement approaches to in vivo cardiovascular research. $\mathrm{Br}$ J Pharmacol 161, 749-754 (2010).

13. De Witt SM, Swieringa F, Cavill R, Lamers MM, Van Kruchten R, Mastenbroek T, Baaten C, Coort S, Pugh N, Schulz A, Scharrer I, Kerstin J, Zieger B, Clemetson KJ, Farndale RW, Heemskerk JW, Cosemans JM. Identification of platelet function defects by multiparameter assessment of thrombus formation. Nat Commun 5, 4257 (2014).

14. Roest M, Reininger A, Zwaginga JJ, King MR, Heemskerk JW. Flow chamber-based assays to measure thrombus formation in vitro: requirements for standardization. J Thromb Haemost 9, 2322-23224 (2011). 
15. Hosokawa K, Ohnishi T, Sameshima H, Miura N, Ito T, Koide T, Maruyama I. Analysing responses to aspirin and clopidogrel by measuring platelet thrombus formation under arterial flow conditions. Thromb Haemost 109, 102-111 (2013).

16. Flamm MH, Colace TV, Chatterjee MS, Jing H, Zhou S, Jaeger D, Brass LF, Sinno T, Diamond SL. Multiscale prediction of patient-specific platelet function under flow. Blood 120, 190-198 (2012).

17. Neeves KB, Onasoga AA, Hansen RR, Lily JJ, Venckunaite D, Sumner MB, Irish AT, Brodsky G, Manco-Johnson MJ, Di Paolo JA. Sources of variability in platelet accumulation on type 1 fibrillar collagen in microfluidic flow assays. Plos One 7, e54680 (2013).

18. Li R, Diamond SL. Detection of platelet sensitivity to inhibitors of COX-1, $\mathrm{P}_{2} \mathrm{Y}_{1}$, and $\mathrm{P} 2 \mathrm{Y}_{12}$ using a whole blood microfluidic flow assay. Thromb Res 133, 203-210 (2014).

19. Stegner D, Nieswandt B. Platelet receptor signaling in thrombus formation. J Mol Med 89, 109-121 (2011).

20. Versteeg HH, Heemskerk JW, Levi M, Reitsma PS. New fundamentals in hemostasis. Physiol Rev 93, 327-358 (2013).

21. Croft D, Mundo AF, Haw R, Milacic M, Weiser J, Wu G, Caudy M, Garapati P, Gillespie M, Kamdar MR, Jassal B, Jupe S, Matthews L, May B, Palatnik S, Rothfels K, Shamovsky V, Song H, Williams M, Birney E, Hermjakob H, Stein L, D’Eustachio P. The Reactome pathway knowledgebase. Nucleid Acids Res 42, D472-477 (2014).

22. Mangin P, Yap CL, Nonne C, Sturgeon SA, Goncalvas I, Yuan Y, Schoenwaelder SM, Wright CE, Lanza F, Jackson SP. Thrombin overcomes the thrombosis defect associated with platelet GPVI/FcRy deficiency. Blood 107, 4346-4353 (2006).

23. Eckly A, Hechler B, Freund M, Zerr M, Cazenave JP, Lanza F, Mangin PH, Gachet C. Mechanisms underlying $\mathrm{FeCl}_{3}$-induced arterial thrombosis. J Thromb Haemost 9, 779-789 (2011).

24. Jupe S, Akkerman JW, Soranzo N, Ouwehand WH. Reactome - a curated knowledgebase of biological pathways: megakaryocytes and platelets. J Thromb Haemost 10, 2399-2402 (2012).

25. Dubois C, Panicot-Dubois L, Gainor JF, Furie BC, Furie B. Thrombin-initiated platelet activation in vivo is vWF independent during thrombus formation in a laser injury model. J Clin Invest 117, 953-960 (2007).

26. Cosemans JM, Kuipers MJ, Lecut C, Louberle ST, Heeneman S, Jandrot-Perrus M, Heemskerk JW. Contribution of platelet glycoprotein VI to the thrombogenic effect of collagens in fibrous atherosclerotic lesions. Atherosclerosis 181, 19-27 (2005).

27. Reininger AJ, Bernlochner I, Penz SM, Ravanat C, Smethurst P, Farndale RW, Gachet C, Brandl R, Siess W. A 2-step mechanism of arterial thrombus formation induced by human atherosclerotic plaques. $J$ Am Coll Cardiol 55, 1147-1158 (2010). 



\section{CHAPTER 9}

GENERAL DISCUSSION 
The goal of this thesis was to untangle the process of thrombus formation in a systematic way. By using novel tools of systems biology, it has been possible to clarify the roles of multiple platelet receptors, interacting with key adhesive substrates of the vessel wall, in thrombus formation under flow. Also by developing scoring tools to compare the process of thrombus formation in vivo and in vitro from published mouse studies, new insights could be obtained with relevance for human cardiovascular disease.

\section{Advances in the measurement of whole blood thrombus formation}

An advantage of the use of flow chamber devices is that they give information on platelet and coagulant functions of one blood sample under flow conditions. The recent introduction of smallsized microfluidic flow chambers is a major step forward, as these need only small blood samples 1-3. As described in chapter 2, the so-called PDMS microfluidic chambers, which are customer made and occur in multiple designs, have the potential of generating high-throughput data on thrombus formation. However, since these chambers differ from laboratory to laboratory (for instance with multi-channels or discontinuous immobilized thrombogenic surfaces), they are only poorly standardized. This also holds for the few commercial flow devices, e.g. the Ibidi, Venaflux, Cellix or Bioflux systems ${ }^{4}$. The conventional Maastricht flow chamber is used in our laboratory in many studies with human and mouse blood ${ }^{5-8}$ and is also employed in other laboratories ${ }^{9}$. In the present thesis, we choose for this chamber in the development of a high-throughput flow assay. As described in chapters 3-4, this resulted in a test in which the activity and thrombus-forming potential of platelets from one individual subject can be tested on an array of microspot surfaces. Moreover, the testing can be performed at both arterial or venous flow shear rates. As such, this test is fast and has a higher throughput than the current golden standard test for platelet function, i.e. light transmission aggregometry. The latter only determines light transmission changes due to platelet shape change and aggregation and operates in the absence of flow.

The developed microspot flow test clearly provides valuable and new information, but it also has some disadvantages. First, it requires the presence of a high-resolution microscope and, hence, operation by experts. Second, some of the parameters (fluorescence markers) still had a relatively high inter-assay variation. Further refinement of the staining protocols will be needed to reduce this variability. A third point of concern is the not always optimal quality of the blood samples - in spite of all precautions taken -, implicating that more attention will need to be paid to preserve platelet functions while drawing the blood.

\section{Thrombus formation unlocked as a multi-receptor and multi-factorial process}

Current knowledge is that platelet interaction with multiple receptors is required for stable adhesion under flow conditions ${ }^{8,10}$. Earlier work has mostly concentrated on the synergy of the collagen receptors, glycoprotein VI (GPVI) and integrin $a_{2} \beta_{1}$, and the von Willebrand factor (VWF) receptors, glycoprotein Ib-V-IX (GPIb) and integrin $a_{\mathrm{IIb}} \beta_{3}$ in thrombus formation $6,9,11,12$. In chapter 4, we applied for the first time systems biology techniques to compare the roles of these receptors with other adhesive platelet receptors in thrombus formation.

Unsupervised hierarchical cluster analysis allowed the systematic comparison of the effects of 52 surfaces interacting with combinations of these four and other adhesive receptors (CLEC-2, $a_{6} \beta_{1}, C D 36, a_{5} \beta_{1}, a_{v} \beta_{3}$ ). The analysis pointed to clustering of the surfaces into three types, i.e. giving rise to the formation of type I, II or III thrombi at high shear rate. As schematized in Figure 1 , type I thrombi are formed on surfaces at which only few platelets adhere. Surfaces forming type II thrombi induce extensive platelet adhesion with intermediate platelet activation and aggregate formation, while surfaces forming type III thrombi produce large aggregates of fully activated platelets. It could be established that the clustering into these three types of thrombi was robust and relatively independent on the selection and composition of the surfaces. This recognition made a following step possible, namely to define which combinations of adhesive proteins - and 
thus which platelet receptors - are required for the formation of full-size, type III thrombi under high-shear flow conditions. Overall, to form full thrombi, we found that a synergy is needed of the high shear-dependent vWF receptor GPIb, the major integrins $a_{2} \beta_{1}, a_{6} \beta_{1}$ or $a_{\mathrm{IIb}} \beta_{3}$ and the signalinglinked receptors GPVI or CLEC-2. Herein, the roles of $a_{5} \beta_{1}, a_{v} \beta_{3}$ and CD36 were relatively minor. In chapters 4 and 6 , we provide several pieces of evidence for a major contribution of platelet granule secretion to the process of thrombus formation. Patients with gray platelet syndrome and Hermansky-Pudlak syndrome are characterized by a deficiency in the presence of a- and dense granules, respectively. In chapter 4 , the defect in gray platelet syndrome was confirmed by a reduced P-selectin expression after thrombus formation. Also in a patient with Hermansky-Pudlak syndrome, thrombus formation was decreased on various surfaces.

Interestingly, a patient with severe combined immunodeficiency (SCID) ${ }^{13}$, associated with an almost complete defect in store-induced operated calcium entry in hematopoietic cells including platelets, showed an increase in CLEC-2 and GPVI-dependent platelet activation, which suggests the presence of a compensatory mechanism for the defective calcium entry. In comparison, in a patient with May-Hegglin anomaly (actin-myosin cytoskeletal defect and macrothrombocytopenia), we found a decrease in mostly GPVI-dependent thrombus formation.

Another relevant finding was the relatively strong role of integrin $a_{6} \beta_{1}$ in thrombus formation. Laminin has shown to be a major extracellular matrix component, supporting the interaction via $a_{6} \beta_{1}$ on platelets ${ }^{14}$. Recent evidence indicates that laminins are also stored in platelets, ${ }^{15}$ implicating that secreted laminin may support the buildup of a thrombus via $a_{6} \beta_{1}$ interactions.

In chapter 6 , we describe a role for the thrombospondin-1 - CD36 axis in collagen-dependent thrombus stabilization and thrombus formation. Similarly to laminin, thrombospondin-1 is also present in the vessel wall and is released from the a-granules in platelets ${ }^{16}$. Using murine $\mathrm{Cd} 36^{-{ }^{-}}$and $\mathrm{Tsp}$ /-platelets, we found that especially platelet procoagulant activity and thrombus stabilization were decreased in the absence of these proteins. In comparison, in Nbeal2 $\%$ mice, which completely lack a-granules, an even stronger effect has been seen, including impaired adhesion, reduced procoagulant activity, diminished thrombus formation and increased thrombus stability ${ }^{17}$. It should be noted in this context that both thrombospondin-1 and CD36 also have other interaction partners than each other. Platelet activation also depends on the activity of the endothelium. For instance, the resting endothelium produces sufficient amounts of prostacyclin and nitric oxide to keep flowing platelets in an inactivated state. The protein vWF plays a more complex role in the interactions of platelets and endothelial cells. Although platelets also contain some VWF, the endothelium is the largest source of this multimeric glycoprotein ${ }^{18,19}$. VWF is cleaved by the plasma protease ADAMTS-13, while thrombospondin-1 can protect VWF from cleavage ${ }^{20}$. Especially long VWF multimers appear to be most adhesive for platelets, if extended at high shear shear on microvascular cells or exposed collagen ${ }^{21}$.

Cleavage of VWF multimers in the A2-domain by the protease ADAMTS-13 takes place in an asymmetric way, resulting in multimers with slower, intermediate or faster migrating triplet bands. The slower migrating (larger size) triplet bands of VWF multimers contain an additional $140 \mathrm{kDa}$ $\mathrm{N}$-terminal peptide fragment, which is missing for the faster migrating triplets ${ }^{18}$. Our data in chapter 5 indicate that a preparation of VWF with extra $\mathrm{N}$-terminal peptide sequence of the A2 domain had a significant higher binding affinity for recombinant glycoprotein Ib. The same fraction was also more active in supporting thrombus formation on collagen under a variety of conditions. These results thus indicate that the larger size VWF triplet bands, containing an extra $\mathrm{N}$-terminal peptide sequence, are most potent in thrombus formation under arterial flow conditions. This is a relevant finding, since changes in the extent of VWF cleavage by ADAMTS-13 may result in von Willebrand disease ${ }^{22}$ or thrombotic thrombocytopenic purpura ${ }^{23}$.

\section{Identifying platelet function defects}

As described in chapter 4, clustering and arranging of the big dataset for 52 thrombogenic surfaces and 8 platelet function outcome parameters led to the identification of the most informative 


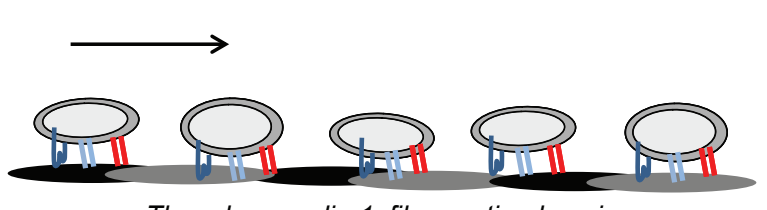

Thrombospondin-1, fibronectin, decorin

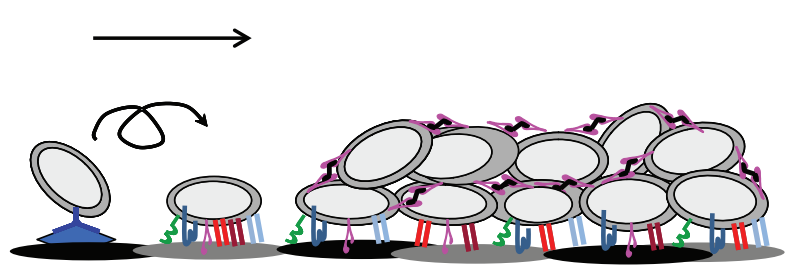

VWF, trombospondin-1, laminin, fibronectin, vitronectin

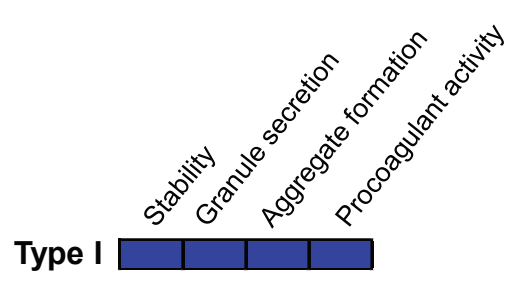

Type II

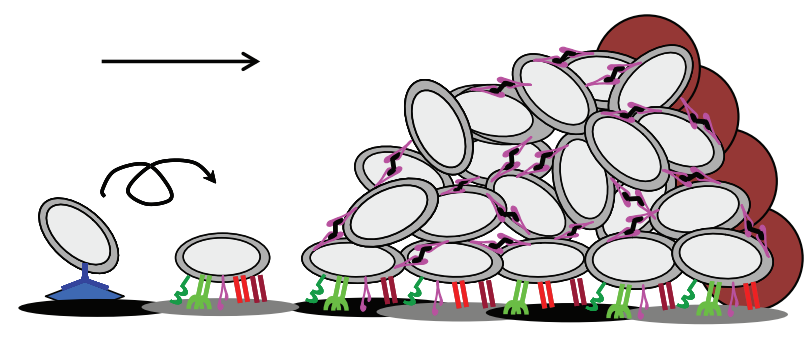

VWF, collagen, rhodocytin, laminin, fibrinogen

Type III

\begin{tabular}{|c|c|c|c|c|}
\hline & $\| \alpha_{5} \beta_{1}$ or $\alpha_{v} \beta_{3}$ & $\propto \mathrm{VWF}$ & $\xi$ GPVI & $\mathcal{I}$ Fibinogen \\
\hline W CD36 & $\| \alpha_{2} \beta_{1}$ & 人 GPIb-V-IX & $\alpha_{11 b} \beta_{3}$ & $\| \alpha_{6} \beta_{1}$ \\
\hline
\end{tabular}

Figure 1. Three types of thrombi formed at high shear rate. Different stimuli, i.e. components that are release from platelet granules, vascular components or shear, trigger the formation of different types of thrombi. Type I thrombi consist of single adhered platelets (gray) with limited activation. Type II and type III thrombi require first interaction of platelet GPIb with VWF. Type II thrombi display intermediate platelet adhesion and activation, evoked by GPVI, CD36 and/or integrins. Type III thrombi show high adhesion and activation via GPVI or CLEC-2 and/or all integrins. Procoagulant platelets (brown) are almost absent in type II thrombi in comparison to type III thrombi. Underneath every thrombus type, the corresponding thrombogenic surfaces are indicated. Left arrow indicates direction of flow. Color bars: blue $=$ low, green $=$ intermediate, red $=$ high .

variables of this process. The cluster analysis resulted in a robust selection of nine surfaces and six parameters, which can be used to determine the involvement of all key platelet receptors in thrombus formation. Establishment of the intra- and inter- individual variation for these $9 \times 6$ test variables pointed out that there was a marked subject-dependent contribution to almost all of these variables. This suggested that aberrant platelet activity in patients with bleeding or a prothrombotic propensity may well be detected. Similar observations have been made by others in flow studies using microfluidics devices. Herein, whole blood from healthy subjects, who received aspirin or ADP inhibitors, was perfused over a collagen surface, and marked inter-individual variation was observed in the amount of platelet deposition before and after intake 24,25 .

As described in the previous section, defective thrombus formation could indeed be detected in several patients with rare bleeding disorders. The most discriminative surfaces in this respect were combinations of VWF with rhodocytin, GFOGER-(GPO) peptide or collagen I. The recording of multiple parameters gave a kind of platelet "phenoprint" of general (platelet aggregation) and specific (platelet P-selectin and phosphatidylserine exposure) responses per type of surface, and hence type of receptors. In chapter 7 we have provided an in-depth overview of the genes that 
play a role in platelet phoshatidylserine exposure during thrombus formation.

The data of chapter 4 accordingly point to a clinical potency of the microspot test. We see a wide range of possible applications, including (i) the detection of increased platelet activity in patients with acute cardiovascular infarction or otherwise increased risk of arterial thrombosis, (ii) the monitoring of the efficacy of antithrombotic treatment of patients, (iii) the identification and treatment of platelet dysfunction or thrombocytopenia, and (iv) the determination of the risk of bleeding in patients undergoing fluid dilution during or after major invasive surgery. A further development of the flow assay would be to make it microscopic-independent. An interesting possibility is to use the newly developed lens-free computational microscopic systems in combination with our microspot flow device ${ }^{26,27}$.

\section{Translational aspects in thrombosis and hemostasis}

Chapter 8 describes a meta-analysis to evaluate the outcomes of published in vivo and in vitro studies of murine thrombus formation at arterial shear stress with the help of a novel calibration method. This provided insight into the roles of 315 mouse genes in arterial thrombosis with or without bleeding, and enabled us to compare the various methods and to identify new human targets for treatment of cardiovascular disease. For the meta-analysis, we used a three-point and five-point scoring scale to compare the effects of genetic modifications in different publications. The five-point scale was more discriminative in distinguishing a moderate or strong increase or decrease in thrombus formation, and appeared to be most valuable when at least two study scores of the same gene were available. Detailed statistical analysis indicated that the type of vascular bed did not influence the study score. In contrast, the type of injury appeared to be of influence, in that the scores for laser and photochemical injury compared well with those of $\mathrm{FeCl}_{3}$ injury, but only at the three-point and not at the five-point scale.

Another relevant finding was that strong correlations were obtained between the scores per gene for studies of in vivo thrombosis (including thromboembolism) and studies of in vitro thrombus formation. This is explaining by the fact that for a considerable part both the in vitro and the in vivo studies are collagen-dependent. Thus, in either case the thrombosis process is triggered by the exposure or presence of collagen fibers. Interestingly, the meta-analysis led to the identification of 44 genes, which played a role in murine arterial thrombus formation, but were not implicated in tail bleeding. Translating these data to a network of human genes, involved in platelet function or cardiovascular disease, showed that most of the 44 genes were located on highly connected nodes, thus suggesting that these can be regulating targets for antithrombotic therapy with a limited role in hemostasis. Such therapy may lead to a reduced risk of cardiovascular disease, without bleeding as unwanted side-effect. Although we must realize that animal models cannot always predict of what is occurring in man ${ }^{28}$, the present meta-analysis clearly indicates that animal research is of advantage in the field of thrombosis and hemostasis.

\section{References}

1. Kent NJ, O'Brien S, Basabe-Desmonts L, Meade GR, MacCraith BD, Corcoran BG, Kenny D, Ricco AJ. Shear-mediated platelet adhesion analysis in less than 100 mul of blood: toward a POC platelet diagnostic. IEEE Trans Biomed Eng 58, 826-830 (2011).

2. Neeves KB, Maloney SF, Fong KP, Schmaier AA, Kahn ML, Brass LF, Diamond SL. Microfluidic focal thrombosis model for measuring murine platelet deposition and stability: PAR4 signaling enhances shear-resistance of platelet aggregates. J Thromb Haemost 6, 2193-2201 (2008).

3. Nesbitt WS, Westein E, Tovar-Lopez FJ, Tolouei E, Mitchell A, Fu J, Carberry J, Fouras A, Jackson SP. A shear gradient-dependent platelet aggregation mechanism drives thrombus formation. Nat Med 15, 665-673 (2009).

4. Roest M, Reininger A, Zwaginga JJ, King MR, Heemskerk JW. Flow chamber-based assays to measure thrombus formation in vitro: requirements for standardization. J Thromb Haemost 9, 2322-2324 (2011).

5. Cosemans JM, Angelillo-Scherrer A, Mattheij NJ, Heemskerk JW. The effects of arterial flow on platelet 
activation, thrombus growth, and stabilization. Cardiovasc Res 99, 342-352 (2013).

6. Munnix IC, Kuijpers MJ, Auger J, Thomassen CM, Panizzi P, Van Zandvoort MA, Rosing J, Bock PE, Watson SP, Heemskerk JW. Segregation of platelet aggregatory and procoagulant microdomains in thrombus formation: regulation by transient integrin activation. Arterioscler Thromb Vasc Biol 27, 2484-2490 (2007).

7. Van Kruchten R, Cosemans JM, Heemskerk JW. Measurement of whole blood thrombus formation using parallel-plate flow chambers - a practical guide. Platelets 23, 229-242 (2012).

8. Versteeg HH, Heemskerk JW, Levi M, Reitsma PH. New fundamentals in hemostasis. Physiol Rev 93, 327-358 (2013).

9. Nieswandt B, Moser M, Pleines I, Varga-Szabo D, Monkley S, Critchley D, Fässler R. Loss of talin1 in platelets abrogates integrin activation, platelet aggregation, and thrombus formation in vitro and in vivo. J Exp Med 204, 3113-3118 (2007).

10. Ruggeri ZM, Mendolicchio GL. Adhesion mechanisms in platelet function. Circ Res 100, 1673-1685 (2007).

11. Savage B, Ruggeri ZM. Selective recognition of adhesive sites in surface-bound fibrinogen by glycoprotein IIb-IIIa on nonactivated platelets. J Biol Chem 266, 11227-11233 (1991).

12. Siljander PR, Munnix IC, Smethurst PA, Deckmyn H, Lindhout T, Ouwehand WH, Farndale RW, Heemskerk. Platelet receptor interplay regulates collagen-induced thrombus formation in flowing human blood. Blood 103, 1333-1341 (2004).

13. Bergmeier W, Oh-Hora M, McCarl CA, Roden RC, Bray PF, Feske S. R93W mutation in Orai1 causes impaired calcium influx in platelets. Blood 113, 675-678 (2009).

14. Schaff M, Tang C, Maurer E, Bourdon C, Receveur N, Eckly A, Hechler B, Arnold C, de Arcangelis A, Nieswandt B, Denis CV, Lefebvre O, Gerogers-Labouesse E, Gachet C, Lanza F, MAngin PH. Integrin $a_{6} \beta_{1}$ is the main receptor for vascular laminins and plays a role in platelet adhesion, activation, and arterial thrombosis. Circulation 128, 541-552 (2013).

15. Pook M, Tamming L, Padari K, Tiido T, Maimets T, Patarroyo M, Juronen E, Jaks V, Ingerpuu S. Platelets store laminins $411 / 421$ and $511 / 521$ in compartments distinct from alpha- or dense granules and secrete these proteins via microvesicles. J Thromb Haemost 12, 519-527 (2014).

16. Nergiz-Unal R, Rademakers T, Cosemans JM, Heemskerk JW. CD36 as a multiple-ligand signaling receptor in atherothrombosis. Cardiovasc Hematol Agents Med Chem 9, 42-55 (2011).

17. Deppermann C, Nurden P, Nurden AT, Nieswandt B, Stegner D. The Nbeal2 ${ }^{-1-}$ mouse as a model for the gray platelet syndrome. Rare Dis 1, e26561 (2013).

18. Fischer $B E$, Thomas KB, Schlokat $U$, Dorner F. Triplet structure of human von Willebrand factor. Biochem J 331, 483-488 (1998).

19. Sonneveld MA, de Maat MP, Leebeek FW. Von Willebrand factor and ADAMTS-13 in arterial thrombosis: a systematic review and meta-analysis. Blood Rev 28, 167-178 (2014).

20. Bonnefoy A, Daenens K, Feys HB, De Vos R, Vandervoort P, Mermylen J, Lawler J, Hoylaerts MF. Thrombospondin-1 controls vascular platelet recruitment and thrombus adherence in mice by protecting (sub)endothelial VWF from cleavage by ADAMTS-13. Blood 107, 955-964 (2006).

21. Turner NA, Nolasco L, Ruggeri ZM, Moake JL. Endothelial cell ADAMTS-13 and VWF: production, release, and VWF string cleavage. Blood 114, 5102-5111 (2009).

22. Michiels JJ, Berneman Z, Gadisseur A, Van der Planken M, Schroyens W, Van de Velde A, Van Vliet H. Classification and characterization of hereditary types 2A, 2B, 2C, 2D, 2E, 2M, 2N, and 2U (unclassifiable) von Willebrand disease. Clin Appl Thromb Hemost 12, 397-420 (2006).

23. Drews K, Seremak-Mrozikiewicz A, Sobieszczyk S, Barlik M. Inherited thrombotic thrombocytopenic purpura in pregnancy. Neuro Endocrinol Lett 34, 508-513 (2013).

24. Li R, Diamond SL. Detection of platelet sensitivity to inhibitors of COX-1, P2Y , and P2Y $\mathrm{Y}_{12}$ using a whole blood microfluidic flow assay. Thromb Res 133, 203-210 (2014).

25. Li R, Fries S, Li X, Grosser T, Diamond SL. Microfluidic assay of platelet deposition on collagen by perfusion of whole blood from healthy individuals taking aspirin. Clin Chem 59, 1195-1204 (2013).

26. Isikman SO, Greenbaum A, Lee M, Bishara W, Mudanyali O, Su TW Ozcan A. Lensfree computational microscopy tools for cell and tissue imaging at the point-of-care and in low-resource settings. Analyt Cell Pathol 35, 229-247 (2012).

27. Noom DW, Eikema KS, Witte S. Lensless phase contrast microscopy based on multiwavelength Fresnel diffraction. Opt Lett 39, 193-196 (2014).

28. Perrin S. Preclinical research: Make mouse studies work. Nature 507, 423-425 (2014). 
SUMMARY 
Platelets play a crucial role in thrombosis and hemostasis. They contribute to the cessation of bleeding by rapid adhesion to the arterial vessel wall at sites of endothelial injury. Uncontrolled platelet activation after rupture or erosion of an atherosclerotic plaque can lead to vessel occlusion and result in a heart attack or stroke. Intravascular thrombus formation is a dynamic process, where platelets adhere to collagen, become activated, secrete, aggregate and contract. Other cells from the blood and vessel wall as well as plasma components like the coagulation factors and the blood flow conditions have a modulating role herein. In this thesis several aspects of the process of thrombus formation have been studied in detail, and systems biology approaches have been used as a strategy to analyze and compare the involvement of the many-fold sub-processes.

Chapter 1 provides relevant background information on platelet activation and thrombus formation. It is described that platelet-adhesive components of the vessel wall other than collagen can also trigger thrombus formation. Yet, the traditional model of thrombus formation is still based on collagen exposure. In addition, a number of adhesive proteins, which are stored in the platelet a-granules such as von Willebrand factor (VWF) and thrombospondin-1, may also play a role in thrombus formation, but this has not been explored. Also addressed is how flow chamber assays can be used to measure thrombus formation, and what the possible clinical relevance is of such assays. The chapter ends with introducing some techniques used in systems biology. This approach has a great potential in the handling of 'big data' in the field of thrombosis and hemostasis.

Commercial and custom-made parallel-plate flow chambers are more and more used for studies of platelet activation and thrombus formation in whole blood at arterial or venous shear rates. Chapter $\mathbf{2}$ highlights the technical progress that has been made by developing microfluidic devices composed of polydimethylsiloxane. With the introduction of this material it has become possible to downscale the dimensions of the flow channels and, thereby, the blood volume needed per flow assay. This chapter furthermore describes that both the commercially available flow devices and the custom designed specimens lack standardization, which impairs their suitability for (pre)clinical testing of thrombus formation. In the following chapters, various directions are proposed for extension and standardization of the conventional flow chamber measurements, for instance by using a defined array of platelet-adhesive surfaces and by employing high-throughput data analysis.

In chapter 3, standardized protocols are provided for usage of the Maastricht parallel-plate flow chamber to assess thrombus formation on different types of platelet-adhesive, thrombogenic surfaces. Detailed information is given on the way of application of adhesive proteins as microspots, the preferential way of blood drawing, the required flow perfusion protocols, as well as lists for trouble shooting. For several (confocal) fluorescence microscopic systems, methods are given for the capturing of brightfield and fluorescence images to determine (stable) platelet adhesion in realtime, and to assess endpoint parameters of thrombus formation, such as the platelet activation status (fibrinogen binding, P-selectin expression and procoagulant activity), and the thrombus size and morphology (morphological score, integrated feature size, platelet deposition and thrombus volume). Relevant details on software packages and macros for image processing and calculations are provided as well.

According to the classical model of collagen-based thrombus formation at high wall-shear rate, initial platelet rolling occurs via the interaction of glycoprotein Ib-V-IX (GPIb) with collagen-bound VWF. Activation of the platelets is accomplished by interplay of the collagen receptors, glycoprotein VI (GPVI) and integrin $a_{2} \beta_{1}$, while platelet-platelet interactions are mediated by integrin $a_{\mathrm{IIb}} \beta_{3}$. However, platelets expose many other adhesive receptors that may also play a role in thrombus formation. In chapter $\mathbf{4}$ we describe the development of a multi-surface and multi-parameter flow chamber assay to characterize the process of thrombus formation by taking into account all abundantly expressed platelet-adhesive receptors. For this assay, we used whole blood from healthy subjects and several patients with platelet function defects. Altogether, we compared 52 adhesive surfaces (with known receptor interactions) and eight output parameters, reflecting 
distinct stages of thrombus formation. Systems biology analytics allowed us to find distinct patterns between these surfaces (i.e., platelet receptors) and output parameters.

Unsupervised hierarchical cluster analysis revealed three types of thrombus formation: type I thrombi formed on surfaces of single-protein coatings, resulting in limited adhesion of few platelets; type II thrombi being formed mostly on surfaces co-coated with VWF, giving deposition of multiple single platelets or small aggregates with limited platelet activation; type III thrombi on only particular, combined surfaces, which led to the formation of large aggregates with highly activated platelets. Principal component analysis indicated a predicted decreasing contribution of adhesive receptors to the formation of type III thrombi at high shear rate in the order of GPVI, CLEC-2 $>$ GPIb $>a_{6} \beta_{1}, a_{I I b} \beta_{3}>a_{2} \beta_{1}$. Further, the contribution of CD36, $a_{v} \beta_{3}$ and $a_{5} \beta_{1}$ to type III thrombus formation at high shear rate was only small.

Multiple regression analysis indicated that, with blood from healthy controls, all eight parameters contribute significantly to the clustering into type I-III thrombus formation. However, six of these parameters appeared to be sufficient for the proper quantification of this process. A set of nine surfaces (VWF co-coated) was then selected - involving all major adhesive receptors - to determine reference values for the six parameters of thrombus formation. Whole-blood samples from a number of patients with rare genetically linked platelet function defects were analyzed for thrombus formation and compared with the control data. Presentation as heatmaps revealed distinct abnormalities per surface and parameter for each of the patients. With all blood samples, altered thrombi were formed on surfaces binding GPIb in combination with CLEC, GPVI and/or $a_{2} \beta_{1}$. In particular, parameters of platelet secretion on surfaces were reduced with blood from patients with a partial lack in a-granules or dense granules. On the other hand, parameters linked to platelet aggregation were reduced with blood from a patient with deficiency in integrin $\mathrm{a}_{\mathrm{IIb}} \beta_{3}$ and a patient with a myosin cytoskeletal defect. In contrast, the heatmap of a patient with severe combined immunodeficiency syndrome showed an increase in parameters of platelet activation, suggesting a compensatory mechanism for absence of one of the calcium entry pathways underlying this disease. Taken together, these findings indicate that the 'fingerprints', obtained by multiparameter and multispot analysis of thrombus formation, can be valuable to predict a prothrombotic or bleeding tendency in clinical settings.

Chapter 4 also describes that, for blood from healthy controls, co-coating with VWF resulted in an increased thrombus-forming potential at high shear rate, but not at low shear rate. This was true for the majority of parameters and types of surfaces tested. In line with this, principle component analysis indicated that the contribution of GPIb to type III thrombus formation was markedly increased at high shear rate versus low shear rate. Interestingly, some surfaces - not containing VWF- became more active at low shear rate. Overall, we could conclude that the VWF-GPIb interaction is by far the most potent axis stimulating platelet adhesion and thrombus formation at arterial shear conditions.

In the Weibel-Palade bodies from endothelial cells and in the platelet a-granules, VWF is stored as ultralarge multimers. Once released from these cells, it can be cleaved by the protease ADAMTS-13 into smaller, less active multimers. This cleavage occurs in an asymmetric way, resul- ting in multimers with slower, intermediate or faster migrating triplet bands that correspond to differences in molecular weights. Only the slower migrating triplet bands (larger molecules) contain an extra $\mathrm{N}$-terminal peptide chain, which includes a GPIb binding site. In chapter 5, we functionally characterized two VWF preparations which were similar in VWF multimeric distribution pattern, but markedly differed in triplet band composition. Binding experiments indicated that the preparation with additional $N$-terminal peptide chain had the highest binding affinity for recombinant GPIb in comparison to other preparations. In agreement with this, flow experiments showed that this preparation increased thrombus formation on collagen in vitro, using reconstituted blood or blood from patients with von Willebrand disease. We concluded that VWF forms lacking larger size triplet bands have a decreased potential to recruit platelets under arterial flow conditions. 
The abundant glycoprotein CD36 is a receptor for one of the most highly expressed proteins stored in the platelet a-granules, namely thrombospondin-1 (TSP1). Yet, the precise role of CD36 in thrombus formation at high or low shear rate is still under debate. Chapter $\mathbf{6}$ describes experiments to elucidate this, using blood from mice deficient in either CD36 or TSP1. It is shown that the releasate from wildtype platelets, but not from TSP1-deficient platelets, augmented the adhesion and phosphatidylserine exposure only in (wildtype) platelets expressing CD36. Moreover, the wildtype releasate enhanced collagen-induced integrin activation and phosphatidylserine exposure, and thereby thrombus formation, under flow conditions in vitro. In agreement with this, less stable thrombi with increased embolization were observed in vivo in mice lacking CD36. Altogether, these results pointed to an anchoring role of platelet-released TSP1 via CD36 in platelet adhesion and collagen-dependent thrombus stabilization. Thus, the TSP1-CD36 tandem appears to be another platelet ligand-receptor axis contributing to the maintenance of a stable thrombus.

Chapter 7 provides an overview on the roles of platelets in control of the coagulation process. Comparison of studies using knockout mice and studies using pharmacological inhibitors (human) showed that collagen-induced phosphatidylserine exposure is regulated by a variety of platelet signalling proteins and membrane receptors, for the major part directly or indirectly involved in $\mathrm{Ca}^{2+}$ signal generation. Furthermore, also several granular proteins or proteins regulating a- and dense granule secretion appear to play a role in phosphatidylserine exposure. Membrane exposure of phosphatidylserine generates an efficient surface for thrombin generation and subsequent fibrin formation, which enables fibrin clot retraction of a thrombus. The data analysis revealed a marked similarity in platelet proteins implicated activation processes seemingly different from clot retraction, such as integrin inside-out and outside-in signalling, establishment of homo-typical platelet-platelet interactions, and actin cytoskeleton organization. Overall, this review indicated that, for collagen-adhered platelets, in part common signalling proteins and pathways contribute to the three hallmarks of platelet-based coagulation, i.e. phosphatidylserine exposure, generation of thrombin and fibrin, and retraction of the fibrin clot.

An expanded overview on mouse studies is given in chapter 8, presenting a meta-analysis on the published effects of gene modification on murine arterial thrombus formation in vivo and in vitro, such in comparison to effects on tail bleeding. We examined a total of 1080 published studies, resulting in a database of 315 mouse genes that have been examined for a role in arterial thrombosis and/or hemostasis. Using a calibrated three- and five-point scale to score the effects of gene modification, we found that, in general, the type of vascular injury but not the vascular bed was a variant parameter influencing the outcome of in vivo studies. Furthermore, we established a strong correlation between the outcome of collagen-dependent in vivo models of arterial thrombus formation and whole-blood in vitro measurements of thrombus formation in flow chambers. The scoring system also identified a total of 44 mouse genes, where a positive contribution to thrombus formation was not accompanied by altered tail bleeding. Using systems biology tools, we were able to construct a network for orthologous human genes which revealed multiple novel targets for antithrombotic therapy. This meta-analysis, quantitatively comparing divergent in vivo and in vitro mouse studies, underlines the high degree of homology of murine and human processes in thrombus formation. It may also provide a contribution to 3R (replacement, reduction, refinement) approaches to reduce animal use for in vivo experimentation.

Chapter 9 discusses the main findings of this thesis as well as the potential clinical relevance of these data. It is argued that the mouse studies and the systems biology tools employed have led to more insight into the complex mechanisms of the dynamic process of thrombus formation. 
SAMENVATTING 
Bloedplaatjes spelen een cruciale rol bij de trombose en hemostase. Als de kleinste cellen in het bloed, helpen bloedplaatjes bij de stelping van bloedingen als gevolg van beschadiging van een bloedvat. In geval van ziekte zorgen bloedplaatjes voor trombusvorming, bijvoorbeeld door ruptuur van een atherosclerotische plaque, met als gevolg afsluiting van het bloedvat en een hartinfarct of beroerte. Trombusvorming is een dynamisch proces dat bepaald wordt door adhesie, activatie, secretie, aggregatie en contractie van bloedplaatjes. Daarnaast spelen ook componenten van het bloedplasma (zoals stollingsfactoren) en componenten van de bloedvatwand een modulerende rol bij de vorming van een trombus. In deze thesis is gebruik gemaakt van nieuwe analysetechnieken, ontwikkeld vanuit de systeembiologie, om het veelzijdige proces van trombusvorming beter te kunnen begrijpen. Hoofdstuk 1 geeft relevante achtergrondinformatie over het proces van bloedplaatjesactivatie en trombusvorming. Het klassieke model van trombusvorming gaat uit van bloedplaatjesinteractie met collageen. Uit de literatuur van de laatste jaren is echter gebleken, dat ook andere adhesieve substraten in de vaatwand een rol kunnen spelen in de trombusvorming. Echter, hier is nog maar weinig over bekend. Verder is het onduidelijk in hoeverre eiwitten, die vrijgemaakt worden uit de opslaggranulae van bloedplaatjes, zoals von Willebrand factor (VWF) en trombospondine-1, kunnen bijdragen aan het proces van trombusvorming. Het eerste hoofdstuk beschrijft verder het gebruik van de flowkamer voor het meten van trombusvorming in geïsoleerd bloed. Tenslotte worden enige principes van systeembiologische analysetechnieken geïntroduceerd.

In-huis ontwikkelde en commercieel verkrijgbare parallel-plaat flowkamers worden meer en meer gebruikt voor het bestuderen van het proces van trombusvorming en bloedplaatjesactivatie in bloed onder gedefinieerde stromingscondities. Hoofdstuk $\mathbf{2}$ beschrijft de vooruitgang die gemaakt is in de ontwikkeling van flowkamers, met name van de zogenaamde microfluidic devices gemaakt van polydimethylsiloxaan, waarmee met kleine bloedsamples gewerkt kan worden. Deze techniek maakt het mogelijk om sneller en efficiënter te testen. Echter de veelvormigheid van microfluidic devices - ongeveer elk laboratorium heeft zijn eigen flowkamer - maakt het lastig om de techniek te standaardiseren, waarmee deze volbloedtest nog maar zelden preklinisch ingezet wordt. Aspecten waar standaardisatie gewenst is, zijn onder meer de stromingscondities van het bloed, het adhesieve oppervlak voor bloedplaatjes en de manier van analyse van de vele testuitkomsten, waaronder microscopische beeldjes.

Voor het gebruik van de conventionele Maastricht flowkamer, waarmee het grootste deel van het onderzoek beschreven in deze thesis is uitgevoerd, zijn in hoofdstuk $\mathbf{3}$ een aantal gestandaardiseerde protocollen beschreven. Dit betreft de uitvoering van een multiparameter volbloedtest voor het bepalen van de trombusvorming op verschillende soorten oppervlakken. Protocols zijn opgesteld voor het coaten van plaatjesadhesieve oppervlakken, de manier van bloedafname, de experimentele opzet, en de wijze van beeldanalyse. Bijgevoegd is ook een actielijst met wat te doen bij technische problemen. Verder beschreven zijn het gebruik van verschillende (confocale) fluorescentiemicroscopen, evenals methoden voor het opnemen van witlicht- en fluorescentiebeeldjes. Uitkomstmaten van deze microscopiebeelden zijn de mate van stabiele adhesie van bloedplaatjes en eindpuntparameters, zoals de morfologische score, de grootte van een trombus en de depositie van bloedplaatjes. Andere uitkomstmaten geven informatie over de mate van bloedplaatjesactivatie, te weten granulaire secretie, fibrinogeenbinding en procoagulante activiteit. Tenslotte zijn enige details vermeld van software-toepassingen en protocollen voor beeldverwerking.

In het klassieke model van trombusvorming op collageen zijn slechts een beperkt aantal bloedplaatjesreceptoren actief. Bij arteriële stromingscondities (hoge afschuifsnelheden) vindt initiële bloedplaatjesadhesie plaats via glycoproteïne Ib-V-IX (GPIb) met VWF, dat gebonden is aan collageen. De bloedplaatjes worden vervolgens geactiveerd door samenspel van de twee collageenreceptoren glycoproteïne VI (GPVI) en integrine $a_{2} \beta_{1}$. Aggregatie van bloedplaatjes vanuit het bloed vindt plaats via de fibrinogeenreceptor integrine $\mathrm{a}_{\mathrm{II}} \beta_{3}$. Bloedplaatjes hebben echter ook verschillende andere receptoren die een rol kunnen spelen bij de trombusvorming. Hoofdstuk 
4 beschrijft de ontwikkeling van een multiparameter volbloedtest, waarbij de trombusvorming gemeten wordt op meerdere adhesieve oppervlakken, die tesamen alle belangrijke bloedplaatjesreceptoren omvatten. Deze test is uitgevoerd met bloedmonsters van gezonde vrijwilligers en van een aantal patiënten met een zeldzame bloedingsziekte. In totaal zijn 52 adhesieve oppervlakken (met bekende receptorinteracties) met elkaar vergeleken, en daarnaast acht uitkomstparameters, representatief voor verschillende fasen van trombusvorming. Vervolgens hebben we bekende systeembiologische technieken gehanteerd om patronen te ontdekken in de trombusvorming voor de verschillende uitkomst-parameters en de soorten van oppervlak.

Clusteranalyse leidde tot een driedeling van de oppervlakken, elk resulterend in een bepaald type van trombus. Oppervlakken waarop type I trombi gevormd werden, lieten alleen adhesie zien van losse bloedplaatjes. Type II trombi werden gevormd op oppervlakken, die meestal bestonden uit VWF in combinatie met een ander eiwit; dit resulteerde in de depositie van vele losse bloedplaatjes of van kleine aggregaten met beperkte plaatjesactivatie. Type III trombi werden gevormd op oppervlakken met bepaalde combinaties van eiwitten; deze gaven grote aggregaten bestaande uit sterk geactiveerde bloedplaatjes. Componentanalyse stelde ons in staat om een schatting te maken van de relatieve bijdrage van de afzonderlijke bloedplaatjesreceptoren aan de vorming van een type III trombus. In afnemende volgorde was deze: GPVI, CLEC-2 $>$ GPIb $>a_{6} \beta_{1}, a_{I I b} \beta_{3}>a_{2} \beta_{1}$. Bij arteriële stromingscondities (hoge afschuifsnelheden) was de bijdrage van de receptoren CD36, $a_{v} \beta_{3}$ en $a_{5} \beta_{1}$ verwaarloosbaar klein.

Regressie-analyse leerde dat alle acht parameters bijdroegen aan de groepering in type I-III trombi, althans bij gezonde proefpersonen. Zes van deze parameters bleken voldoende voor een volledige kwantificering van dit proces. Op basis van deze analyse zijn negen van de meest relevante oppervlakken (met gecoat VWF) geselecteerd voor de bepaling van referentiewaarden betreffende zes parameters. Bloedsamples van verschillende patiënten met zeldzame plaatjesgebonden bloedingsaandoeningen werden geanalyseerd op trombusvorming en vergeleken met de controle-data. Zogenoemde heatmaps werden gemaakt voor elk van de patiënten, om inzicht te krijgen in het patroon van afwijkingen per oppervlak en parameter. Bij alle patiëntenmonsters waren er veranderingen in trombusvorming op oppervlakken voor GPIb-adhesie (VWF) in combinatie met CLEC, GPVI en/of $a_{2} \beta_{1}$. Met name parameters van secretie waren verlaagd in bloed van patiënten met partieel granulaire defecten. Anderszins waren parameters van bloedplaatjesaggregatie verlaagd in bloed van een patiënt met integrin $a_{\text {IIb }} \beta_{3}$ deficiëntie en een patiënt met een myosine-celskelet defect. De heatmap van een patiënt met gecombineerd immuundeficiëntie-syndroom toonde daarentegen een toename in sommige activatie-parameters. Samengevat laten deze bevindingen zien dat de fingerprints, verkregen door multiparameter en multi-oppervlakmetingen van trombusvorming, waardevol kunnen zijn bij het klinisch voorspellen van een trombotische of bloedingsneiging.

In hoofdstuk 4 is tevens beschreven dat combinaties van oppervlakken met daarin VWF een sterkere trombusvorming laten zien bij hoge afschuifsnelheden dan bij lage afschuifsnelheden. Dit is in overeenstemming met berekeningen dat GPIb een grote bijdrage levert tot de vorming van type III trombi, doch alleen bij hoge afschuifsnelheden. Daarmee hebben we kunnen aantonen de VWF-GPIb interactie de meest belangrijke is voor bloedplaatjesadhesie en trombusvorming onder arteriële stromingscondities.

Het eiwit VWF komt voor als zeer grote multimeren in de Weibel-Palade lichaampjes van endotheelcellen en in de a-granulae van bloedplaatjes. Na vrijzetting in de bloedstroom knipt het matrixmetalloprotease ADAMTS-13 deze grote multimeren in kleinere en daarmee minder actieve multimeren. Deze proteolyse gebeurt op asymmetrische wijze, wat resulteert in zogenaamde tripletvormen van VWF multimeren met kleine verschillen in molecuulgewicht. De grotere tripletvormen hebben een langere $\mathrm{N}$-terminale zijde met daarin een extra GPIb-bindingsplaats. In hoofdstuk $\mathbf{5}$ zijn twee VWF-preparaten, die verschilden in samenstelling van tripletten, functioneel gekarakteriseerd. Uitvoerige analyse toonde aan dat het VWF-preparaat met extra $\mathrm{N}$-terminus een hogere 
bindingsaffiniteit voor GPIb had dan het andere preparaat. Verhoogde plaatjesadhesieve werking van het eerste preparaat werd bevestigd middels flowkamerexperimenten over collageen onder arteriële stromingscondities, evenals met bloed van patiënten met de ziekte van von Willebrand. Met deze resultaten tonen we aan dat een veranderde tripletsamenstelling, zoals soms aanwezig in deze patiënten, kan resulteren in veranderde bloedplaatjesadhesie onder stromingscondities.

Het glycoproteïne CD36 wordt gezien als dé receptor van een eiwit dat sterk tot expressie komt in de a-granulae van bloedplaatjes, namelijk trombospondine-1 (TSP1). De precieze rol van CD36 en TSP1 bij de trombusvorming onder stromingscondities is echter onduidelijk. Hoofdstuk 6 beschrijft experimenten om hierin opheldering te brengen, gebruikmakend van bloed van muizen met een deficiëntie in ofwel CD36 ofwel TSP1. Aangetoond is dat het secretieproduct van normale bloedplaatjes, maar niet van bloedplaatjes zonder TSP1, de adhesie en fosfatidylserine-expressie verhoogt van bloedplaatjes mits deze ook CD36 bevatten. Bovendien verhoogde het secretieproduct van normale bloedplaatjes de collageengestimuleerde integrine-activatie en fosfatidylserine-expositie tijdens de trombusvorming onder stromingscondities. In overeenstemming hiermee bleken er in vivo minder stabiele trombi gevormd te worden met meer embolisatie in muizen met een CD36-deficiëntie. Tesamen duiden deze resultaten op een verankerende rol van plaatjes-gesecreteerd TSP1 via CD36 bij de adhesie van bloedplaatjes en de collageenafhankelijke trombusstabilisatie. Daarmee wordt de TSP1-CD36 interactie gezien als een volgende bloedplaatjes ligand-receptor as, die kan bijdragen tot de stabiliteit van een trombus.

Hoofdstuk 7 beoogt meer inzicht te geven in de rol van bloedplaatjes bij de bloedstolling. Op basis van analyse van gepubliceerde studies, uitgevoerd met genetisch gemodificeerde muizen en met farmacologische remmers, laten we zien dat de fosfatidylserine-expositie van bloedplaatjes gestimuleerd met collageen gereguleerd wordt door een veelvoud aan signaleringseiwitten en membraanreceptoren, merendeels direct of indirect betrokken bij de calciumsignaalgeneratie. Verder blijken ook regulerende eiwitten van de secretie van $a$ - and $\delta$-granulae alsmede diverse granulaire eiwitten van belang te zijn voor de fosfatidylserine-expositie. Het fosfatidylserine-exposerend membraan biedt een functioneel oppervlak voor trombine- en fibrinevorming, en daarmee voor de fibrine-afhankelijke retractie van een trombus. Onze data-analyse toont verder een opmerkelijke overeenkomst tussen de plaatjeseiwitten betrokken bij ogenschijnlijk zo uiteenlopende processen als stolselretractie, integrine-signalering, vorming van homotypische plaatjes-plaatjes interacties en actine-celskelet organisatie. Samengevat laat deze review zien dat, bij de collageen-adhesie van bloedplaatjes, ten dele gemeenschappelijke signaleringseiwitten en gezamenlijke activeringpaden zorgen voor de drie kenmerken van de bloedplaatjesafhankelijke stolling, te weten fosfatidylserine-expositie, vorming van trombine en fibrine, en retractie van het stolsel.

Een uitgebreid overzicht over muizenstudies is opgenomen in hoofdstuk 8. Uitgewerkt hierin is een meta-analyse van gepubliceerde effecten van genetische modificatie op arteriële trombusvorming in vivo en in vitro, dit in vergelijking met effecten op de staartbloeding. In deze meta-analyse zijn 1080 studies opgenomen, resulterend in een database van 315 muizengenen, die onderzocht zijn op een rol in arteriële trombose en/of hemostase. Gebruikmakend van een gecalibreerde driepunts- en vijfpuntsschaal, hebben wij de effecten van genetische modificatie zo goed en volledig mogelijk in kaart gebracht. Daarbij blijkt dat de manier van vasculaire beschadiging een sterker variante parameter is voor het gen-effect op de trombusvorming dan het vaatbed van beschadiging. Verder vinden wij een sterke correlatie tussen de uitkomst van in vivo trombosestudies en die van in vitro studies met flowkamers, met name bij de collageen-afhankelijke trombosemodellen. De scores laten verder zien dat er in totaal 44 genen zijn, waarbij een positieve bijdrage aan de trombusvorming niet gepaard gaat met een veranderde staartbloeding. Door gebruik te maken van systeembiologische technieken konden wij een netwerk construeren van overeenkomstige humane genen, met daarin diverse nieuwe genen en eiwitten die een doel kunnen zijn voor antitrombotische therapie. Deze meta-analyse bevestigt de hoge mate van homologie tussen muis en mens van de processen en eiwitten betrokken bij de trombusvorming. Verder kan 
deze systematische vergelijking van in vivo en in vitro studies bijdragen tot de $3 \mathrm{~V}$ alternatieven van proefdieronderzoek (vervanging, vermindering, verfijning).

In het afsluitend hoofdstuk 9 zijn de meest belangrijke bevindingen van dit proefschrift bediscussieerd in het licht van de huidige literatuur en de mogelijke klinische relevantie. Toegelicht wordt hoe zowel de muizenstudies als de toegepaste systeembiologische analysemethoden meer inzicht bieden in de complexiteit van de trombusvorming. 



\section{DANKWOORD}


Tot slot mijn dank aan iedereen die mij gesteund heeft de afgelopen jaren. Als promovendus is niets fijner dan om terug te mogen vallen op een plaatjesgroep die enorm motiverend werkt. En niet alleen zij, maar ook alle andere collega's hebben gezorgd voor een goede sfeer.

Allereerst bedankt aan mijn co-promotor en promotor. Beste Judith, bedankt voor alles! Bedankt dat de deur altijd openstond, voor de tijd die je altijd voor mij had maar ook voor je positief, kritisch opbouwende houding die mij menigmaal verder heeft geholpen. Beste prof. Heemskerk, beste Johan, dank dat ik altijd bij je binnen kon lopen. Die drempel is nooit hoog geweest, wat ik echt als heel prettig heb ervaren. Dank voor jullie enorme steun en inzet!

Mijn directe collega's, een speciaal woord van dank voor alle gezellige momenten samen! Al die uitstapjes en etentjes hebben, naast het feit dat ik een aantal kilootjes rijker ben geworden, ook een bijdrage geleverd aan onze "wall-of-fame"! Maar bovenal wil ik jullie bedanken voor al jullie hulp, steun, inzet en niet te vergeten vrolijk- en gezelligheid! Zonder jullie was dit boekje niet tot stand gekomen!

Nadine, dank je voor alle gezelligheid en vriendschap! We hebben samen heel wat afgelachen de afgelopen jaren, zo erg zelfs, dat onze kamer op een gegeven moment de "lachkamer" werd genoemd! Maar we hebben ook vaak de mindere en serieuzere dingen in het leven met elkaar gedeeld. Time to grow up! Super gaaf dat je mijn paranimf wilt zijn en we gaan op naar jouw promotie, klinisch chemicus in spe :-)! Dan mijn andere (ex)roomies: Constance, Jilke, Erik, Roosje, Karen en Magdi, ik heb het erg leuk gevonden om bij jullie op de kamer te hebben gezeten. Even voor de duidelijkheid natuurlijk: jullie zitten voorlopig nog even aan mij vast! Bedankt voor alle fijne gesprekken en heel veel succes met alles wat nog op jullie (promo-)pad komt! Constance, speciaal wil ik jou bedanken voor al je hulp, inzet en gezelligheid! Ik heb natuurlijk ook geleerd dat onze snoeppot niet veilig is voor jou! ;) Magdi, you just started your PhD.. Sok sikert a promóciós! (Just blaim Google Translate in case I speak abracadabra.)

Frauke en Tom, zonder jullie humor en jullie gedrevenheid op het werk was mijn PhD traject niet zo verlopen zoals het nu is gegaan. Bedankt voor jullie tomeloze inzet en jullie positiviteit! Heel veel succes met de afronding van jullie PhD! En Frauke, die CD moeten we toch echt gaan opnemen! Tom, waarschijnlijk is dit het goede moment om mij uit de aio-app te verwijderen! :D Siamack, je bent altijd erg gedreven en er is geen twijfel over mogelijk, maar jij komt er wel! Heel veel succes met het afronden met je PhD! Dan Marion en Marijke. Jullie horen gewoon bij het meubilair van de plaatjesgroep! Ik kan mij de groep niet zonder jullie voorstellen. Bedankt dat ik met wat dan ook altijd op jullie terug kon vallen. Paola, ook bij jou als Johans part-timer, kon ik altijd binnenlopen. Bedankt voor alle leuke gesprekken en gezelligheid! Remco en Ankie, jullie zijn twee van de laatste aanwinsten van de plaatjes groep. Ik ben blij jullie te kennen! Remco, je bent net vader geworden, heel veel geluk samen met je jonge gezinnetje! Ankie, jij nog heel veel succes met je promotie!

Dan mijn oud-collegaatjes Roger en Moniek. Roger, je bent nu een leven aan het opbouwen met je eigen gezin daar ergens hoog in het noorden. Bedankt voor al je hulp en humor toen je nog lid was van de plaatjesgroep, maar ook daarna. Heel veel geluk verder! Moniek, ik kan me de dagen met jou op het lab nog goed herinneren. Al die dagen met eindeloze flowproeven die we samen deden zal ik niet vergeten! En vooral die muzikale begeleiding van jou zal mij daarbij ook altijd bijblijven! Heel veel succes met je verdere carrière als wiskunde lerares! Ik ben blij dat je je weg gevonden hebt! Simone, als groep kregen we jou cadeau voor de entertainment tijdens de lunch! Je kenmerkt jezelf met altijd spannende en humoristische verhalen! Bedankt voor de ontspanning die je met je meebracht! 
Ook wil ik alle medewerkers van de vakgroep Biochemie bedanken voor de fijne sfeer en gezelligheid op de afdeling en daarbuiten. De vele dagjes uit, pizzaparty's en de opening van het academisch jaar zullen mij altijd bij blijven (heus niet alleen vanwege het indische eten, hoor)! Fijn en bijzonder dat we zo met z'n allen op deze manier collega's konden zijn!

I would also like to thank all my collaborators abroad. Dear Prof. Watson, dear Steve, thank you that you gave me the opportunity to visit your lab for a couple of weeks! I have met a great group of colleagues over there! I say thank you to all of Steve's labmembers! Birte, also thanks for the great cooperation with your group in Berlin! I like the friendship that we have and sure I will come to Berlin some day! :- Dear prof. Zieger, dear Barbara, thank you for the nice collaboration. Your input means a lot for the research that we do to develop the flow chamber technique even further!

There are also some (PhD) students that visited our lab during the last five years. Dear Lina, dear Sam great to have met you! I wish you all the best in the future! Ook wil ik Anna bedanken. Jouw stage heeft de hele systems biology in onze onderzoeksgroep zeker een boost gegeven!

Ook nog een speciaal woord van dank aan alle co-auteurs die ik nog niet eerder in dit dankwoord genoemd hebt. Thank you for all your contributions to the different chapters of this thesis. Without all your help I wouldn't have been able to get that far.

Mijn onderzoek was echt nergens geweest zonder al die vrijwilligers die de afgelopen jaren bloed hebben gedoneerd voor mijn onderzoek. Hartstikke bedankt aan iedereen!

Ook wil ik iedereen in mijn vriendenkring bedanken voor alle interesse en bovenal steun op velerlei wijzen! Voor alle momenten van ontspanning en ontlading! Ik ga geen namen noemen, want $\mathrm{ik}$ ben bang dat ik mensen ga vergeten. Daarom zeg ik tegen jullie (je weet dat ik jou bedoel), bedankt voor alle sushi, voor al het Griekse eten, voor al die bezoekjes aan de Mac, voor alle wandelingen, uitstapjes, voor al die oneindige romantische films en actiefilms die we keken, voor al die openhartige en intense gesprekken waar we samen konden lachen en huilen (zelfs aan de telefoon i)), voor die gezellige meidenweekenden, voor dat dansje in de gang bij de keuken, voor al die vrijdagen friet of die vrijdagen dat we zelf wat in elkaar flansten, voor al dat lekkere Indonesische eten en dat ene joekelige ijsje in Epen, voor dat gezellige weekendje in Engeland en al die tijd dat je achter je Macbook voor mij zat... Sjonge! Zonder jullie allemaal was het niet zo gelopen als het gelopen is! :) Dank jullie wel!

Last but certainly not least. Mijn gekke familie... :) Mama, papa, Marloes, Marjolein en Jeroen in het kwadraat, Sophietje, Lien en Jortje. Zonder jullie grenzeloze steun en vertrouwen was ik nu niet zo ver gekomen! Ik kan nu wel veel en mooie woorden zoeken om jullie proberen te bedanken, maar zelfs die zullen tekort aan alles doen, dus laat ik het zo. Hoewel, dat etentje houden jullie tegoed hoor! :)

\section{KORTOM: THANKS TO YOU ALL!}





\section{CURRICULUM VitaE}


Susanne de Witt was born on July 17th 1986 in Brunssum. In 1998 she started secondary school at the Sint Janscollege in Hoensbroek where she received her Gymnasium degree (Economics \& Society) in 2004. She studied Health Sciences at Maastricht University. In 2007 she finished her Bachelor on Bioregulation \& Health and Movement Sciences. She continued her education with the Research master Cardiovascular Biology \& Medicine which she successfully finished in 2009. On the 1st of January 2010 she started as a PhD student at the Department of Biochemistry of the Cardiovascular Research Institute (CARIM) at Maastricht University. She performed research described in this thesis under direct supervision of Prof. Dr. J.W.M. Heemskerk en Dr. J.M.E.M. Cosemans. During her PhD period she visited various international conferences in Cardiff, Liverpool, Birmingham (United Kingdom), Utrecht, Amsterdam and Koudekerken (The Netherlands). For this purpose she received a Young Investigators Award in 2012. Part of her research she performed at the laboratory of Prof. Dr. S.P. Watson (Birmingham, United Kingdom), for which she received a grant of the Coen Hemker Foundation. In August 2014 she started the Kootstra Fellowship (intended for talented $\mathrm{PhD}$ students) as a postdoctoral researcher. 
Susanne de Witt werd geboren op 17 juli 1986 te Brunssum. In 1998 begon ze haar middelbare schoolopleiding aan het Sint Janscollege te Hoensbroek. Ze behaalde haar Gymnasiumdiploma (Economie \& Maatschappij) in 2004. Vervolgens startte ze met de opleiding Gezondheidswetenschappen aan de Universiteit Maastricht. Ze voltooide haar Bachelor, met de specifieke richtingen Bioregulatie \& Gezondheid en Bewegingswetenschappen in 2007. Ze vervolgde deze opleiding met de onderzoeksmaster Cardiovascular Biology \& Medicine, die ze in 2009 met succes afrondde. Op 1 januari 2010 begon ze als PhD student bij de vakgroep Biochemie van het Cardiovascular Research Institute (CARIM) aan de Universiteit van Maastricht. Het onderzoek dat zij verrichtte en wat beschreven staat in dit proefschrift heeft onder de directe begeleiding van Prof. Dr. J.W.M. Heemskerk en Dr. J.M.E.M. Cosemans plaats gevonden. Tijdens deze ruim vierjarige periode presenteerde ze haar onderzoek op internationale congressen in Cardiff, Liverpool, Birmingham (Engeland), Utrecht, Amsterdam en Koudekerken (Nederland). In 2012 werd ze hiervoor onderscheiden met een Young Investigators Award. Een gedeelte van haar onderzoek verrichtte zij op het laboratorium van Prof. Dr. S.P. Watson (Birmingham, Engeland) waarvoor ze tevens een subsidie verkreeg van het Coen Hemker Fonds. In augustus 2014 startte ze als postdoctorale onderzoeker met de Kootstra Fellowship, dat in het bijzonder bedoeld is voor getalenteerde PhD studenten. 



\section{PUBLICATIONS}




\section{Papers}

1. Cosemans JM, Schols SE, Stefanini L, de Witt SM, Feijge MA, Hamulyák K, Deckmyn H, Bergmeier W, Heemskerk JW. Key role of glycoprotein Ib/V/IX and von Willebrand factor in platelet activation-dependent fibrin formation at low shear flow. Blood 2011; 117:651-60.

2. Westein E, de Witt SM, Lamers M, Cosemans JM, Heemskerk JW. Monitoring in vitro thrombus formation with novel microfluidic devices. Platelets 2012; 23:501-9.

3. Fuchs B, de Witt SM, Solecka BA, Kröning M, Obser T, Cosemans, JM, Schneppenheim R, Heemskerk JW, Kannicht C. Distinct role of von Willebrand factor triplet bands in glycoprotein Ib-dependent platelet adhesion and thrombus formation under flow. Semin Thromb Hemost 2013; 39:306-14.

4. Nergiz-Ünal R, Kuijpers MJ, de Witt SM, Heeneman S, Feijge MA, Garcia Caraballo SC, Biessen EA, Haenen GR, Cosemans JM, Heemskerk JW. Atheroprotective effect of dietary walnut intake in ApoE-deficient mice: involvement of lipids and coagulation factors. Thromb Res 2013; 131:411-7.

5. Kuijpers MJ, de Witt SM, Nergiz-Unal R, van Kruchten R, Korporaal SJ, Verhamme P, Febbraio M, Tjwa M, Voshol PJ, Hoylaerts MF, Cosemans JM, Heemskerk JW. Supporting roles of platelet thrombospondin-1 and CD36 in thrombus formation on collagen. Arterioscler Thromb Vasc Biol 2014; 36(6): 1187-92.

6. De Witt SM, Verdoold R, Cosemans JM, Heemskerk JW. Insights into platelet-based control of coagulation. Thromb Res 2014; 133 Suppl2: S139-48.

7. De Witt SM, Swieringa F, Cavill R, Lamers MM, van Kruchten R, Mastenbroek T, Baaten C, Coort S, Pugh N, Schulz A, Scharrer I, Jurk K, Zieger B, Clemetson KJ, Farndale RW, Heemskerk JW, Cosemans JM. Identification of platelet function defects by multi-parameter assessment of thrombus formation. Nat Commun 2014; 5: 4257

8. De Witt SM, Swieringa F, Heemskerk JW, Cosemans JM. Multi-parameter assessment of thrombus formation on microspotted arrays of thrombogenic surfaces. Nature Protocol Exchange, 2014; DOI: ISSN 2043-0116.

9. De Witt SM, Meacham S, Feijge MA, Baaten CC, Jupe S, Turro E, Wiese A, Akkerman JW, Ouwehand WH, Prins MH, Cosemans JM, Heemskerk JW. Genes in arterial thrombus formation and bleeding: translation of meta-data from mouse studies. Submitted.

\section{Abstracts}

1. De Witt SM, Lamers MM, Nergiz-Unal R, Swieringa F, Westein E, Cosemans JM, Heemskerk JW. Platelet receptors in thrombus formation on spotted microarray surfaces. XXIIIth Congress of the International Society on Thrombosis and Hemostasis (ISTH), Kyoto, Japan, 2011. J Thromb Haemost, Suppl. July 2011.

2. De Witt SM, Lamers MM, Swieringa F, Westein E, Farndale RW, Clemetson KJ, Cosemans, JM, Heemskerk JW. Platelet receptors in thrombus formation on spotted microarray surfaces: a role for CLEC-2. Proc. 13 $3^{\text {th }}$ United Kingdom (UK) Platelet Meeting, Cardiff, UK, 2011.

3. De Witt SM, Nergiz-Ünal R, Cosemans JM, Jupe S, Prins M, Heemskerk JW. Analysis of platelet genes in arterial thrombus formation. Proc. Keystone Symposium on Molecular and Cellular Biology "Omics Meets Cell Biology", Alpbach, Austria, 2011.

4. De Witt SM, Lamers MM, Swieringa F, van Kruchten R, Farndale RW, Clemetson KJ, Cosemans JM, Heemskerk JW. Multiparameter assessment of thrombus formation: a systems biology approach. Proc. $1^{\text {st }}$ EUPLAN Conference, Maastricht, The Netherlands, 2012.

5. Fuchs B, de Witt SM, Solecka B, Kroning M, Schwarz B, Obser T, Cosemans JM, Schneppenheim R, Heemskerk JW, Kannicht C. Role of Von Willebrand factor triplet bands in glycoprotein Ib-dependent platelet adhesion under flow conditions. $54^{\text {th }}$ American Society of He- 
matology (ASH) Annual Meeting, Atlanta USA 2012. Blood, Suppl. November 2012.

6. De Witt SM, Lamers MM, Swieringa F, van Kruchten R, Mastenbroek T, Coort S, Cavill R, Pugh N, Farndale RW, Clemetson KJ, Heemskerk JW, Cosemans JM. Advanced multi-parameter assessment of microspot thrombus formation. XXIVth Congress of ISTH, Amsterdam, The Netherlands, J Thromb Haemost, Suppl. June 2013.

7. De Witt SM, Nergiz-Unal R, Feijge MA, Wiese A, Jupe S, Prins MH, Cosemans JM, Heemskerk JW. Genes in arterial thrombus formation: a comparison of in vivo and in vitro studies. XXIVth congress of ISTH, Amsterdam, The Netherlands 2013. J Thromb Haemost, Suppl. June 2013.

8. De Witt SM, Nergiz-Ünal R, Feijge MA, Wiese A, Jupe S, Prins MH, Cosemans JM, Heemskerk JW. Genes in arterial thrombus formation: comparing in vivo and in vitro studies. Proc. $14^{\text {th }}$ UK Platelet Meeting, Birmingham, UK, 2013.

9. Lombard S, Bem D, Gissen P, de Witt SM, Pollitt AY, Watson SP. Mouse CLEC-2 can support adhesion to mouse podoplanin under arterial flow. Proc. 26 $6^{\text {th }}$ UK Adhesion Meeting, Birmingham, UK, 2013.

10. Kuijpers MJ, de Witt SM, Sabrkhany S, oude Egbrink MG, Griffioen A, Cosemans JM, van der Meijden PE, Heemskerk JW. Screening of platelet activation and thrombus formation in prothrombotic disease states. Proc. $5^{\text {th }}$ Internat. Meeting on Angiogenesis, Amsterdam, The Netherlands, 2014.

11. Mattheij NJ, de Witt SM, Nakamura L, Mastenbroek T, Cosemans JM, Zieger B, Heemskerk JW. Human platelet STIM1 and ORAI1 are essential in store-operated calcium entry and thrombus formation at venous conditions. Proc. $2^{\text {nd }}$ EUPLAN Conference, Le Bischenberg, France, 2014.

\section{Oral presentations}

1. De Witt SM. Platelet receptors in thrombus formation on spotted microarray surfaces: a role for CLEC-2. 13 ${ }^{\text {th }}$ UK Platelet Meeting, Cardiff, UK (September 2011).

2. De Witt SM. Development of flow chamber test with microspot array of thrombogenic surfaces. CTMM Meeting Innovative Coagulation Diagnostics, Utrecht (January 2011).

3. De Witt SM. Synergy of platelet receptors in thrombus formation: a microarray approach. $58^{\text {th }}$ Meeting of Scientific \& Standardization Committee, Liverpool, UK (June 2012).

4. De Witt SM. Advanced multi-parameter assessment of microspot thrombus formation: a systems biology approach. Symposium of Netherlands Society on Thrombosis and Haemostasis, Koudekerken (April 2013).

5. De Witt SM. Invited lecture. Advanced multiparameter assessment of microspot thrombus formation: a systems biology approach. Birmingham University, Birmingham, UK (September 2013).

6. De Witt SM. Invited moderator postersessie. XXIVth Congres of International Society on Thrombosis and Hemostasis, Amsterdam, The Netherlands (June 2013).

\section{List of awards/grants}

1. Young Investigators Award. $58^{\text {th }}$ Meeting of Scientific \& Standardization Committee (SSC), Liverpool, UK (June 2012).

2. Coen Hemker Foundation Grant. Research at Institute of Biomedical Research, The Medical School, University of Birmingham, UK (prof. SP. Watson; September-October 2013).

3. Kootstra Fellowship Grant. Postdoctoral Research at Cardiovascular Research Institute (CARIM), University of Maastricht, The Netherlands (prof. JH. Heemskerk / dr. JM. Cosemans; June 2014 - August 2015). 


\section{Publications in newspapers/magazines}

1. Telegraaf. Trombose snel getraceerd met nieuwe test. The Netherlands (July 2014).

2. RTLZ Toekomstmakers. Trombose binnen een paar minuten op te sporen. The Netherlands (July 2014).

3. CTMM magazine. Snellere opsporing bloedingen en trombose mogelijk door nieuwe test. The Netherlands (September 2014).

4. Artsenkrant. Bloeding- en trombose gevoelige patiënten sneller opsporen. Belgium (September 2014). 


\section{SUPPLEMENTAL DATA}




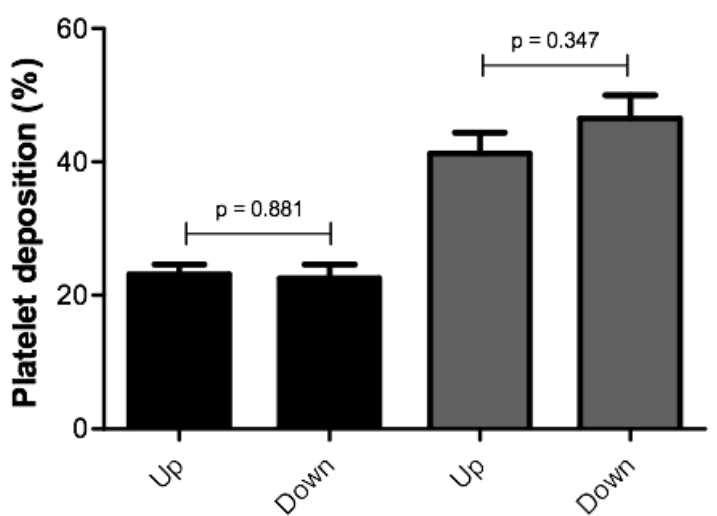

Fibrinogen
Collagen I

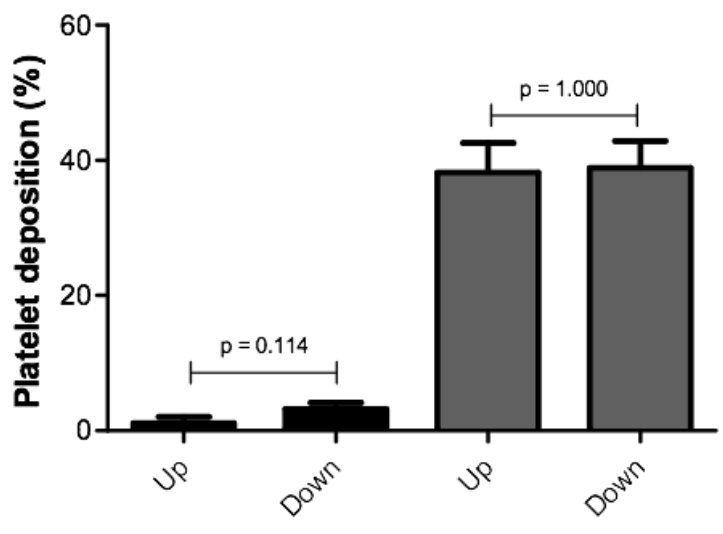

Fibronectin
Collagen I

Supplemental Figure 1. Similar platelet adhesion to microspots of fibrinogen or fibronectin located upstream or downstream of collagen. Whole-blood was perfused for $3.5 \mathrm{~min}$ at $1600 \mathrm{~s}^{-1}$ over microspots containing fibrinogen, fibronectin or collagen I. Coating of microspots was at upstream or downstream locations, as indicated. Platelet deposition was analyzed as surface-area-coverage. Data are mean \pm S.D $(n=8$; Mann-Whitney U test). 


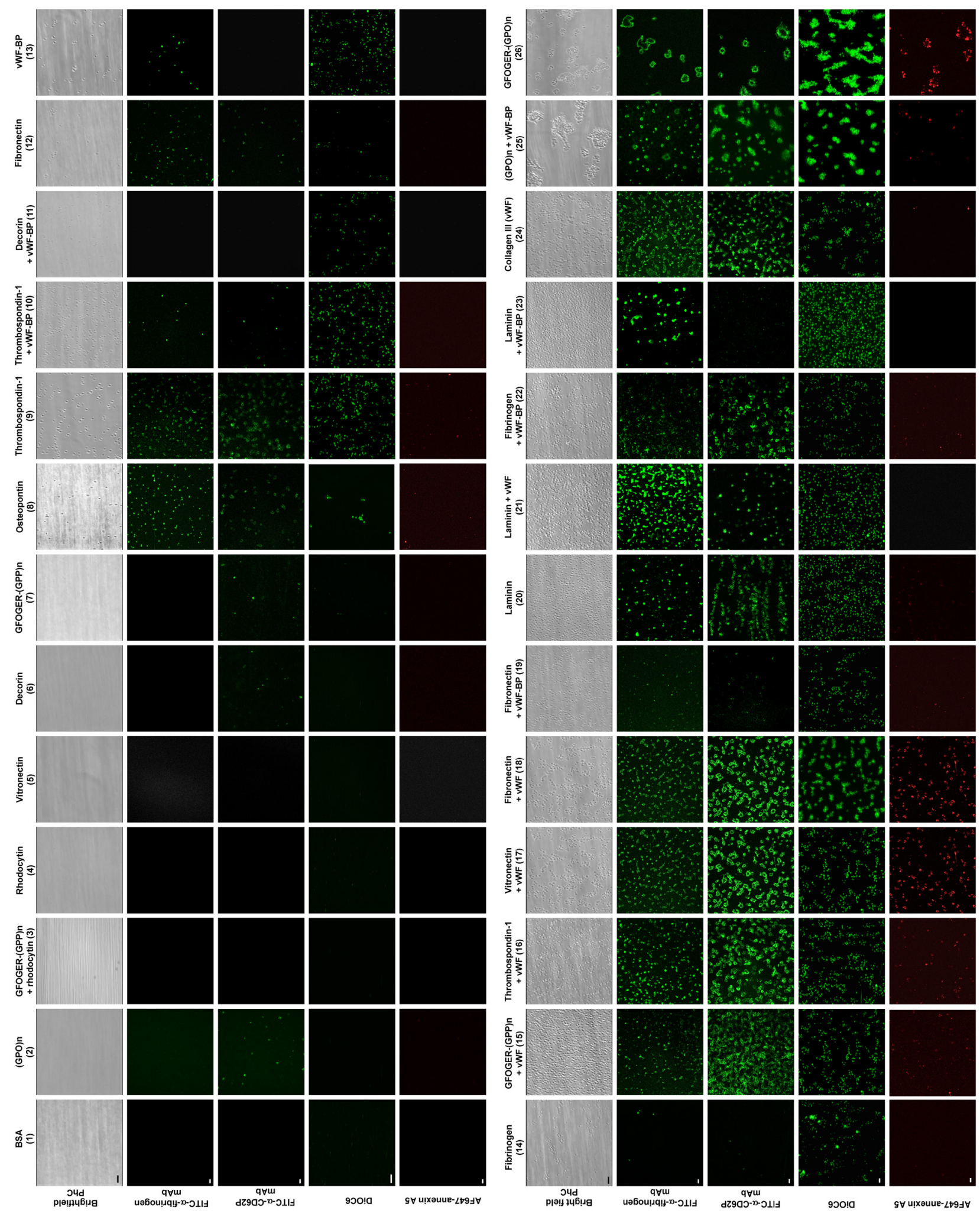

Supplemental Figure 2. Wall chart of representative microscopic images of platelet adhesion and thrombus formation on 52 microspot surfaces. 


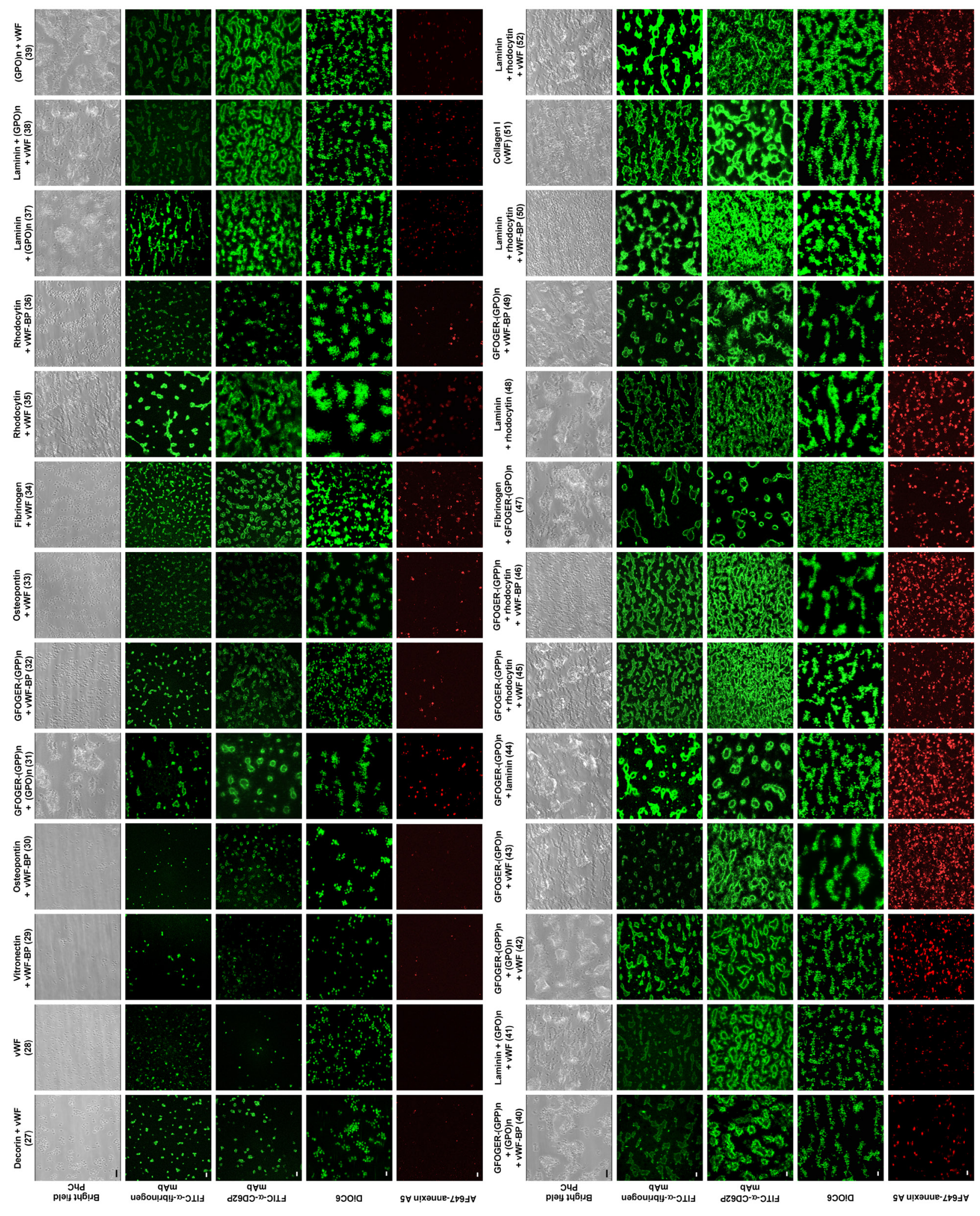

Supplemental Figure 2. Wall chart of representative microscopic images of platelet adhesion and thrombus formation on 52 microspot surfaces. 
Supplemental Figure 2. Wall chart of representative microscopic images of platelet adhesion and thrombus formation on $\mathbf{5 2}$ microspot surfaces. Whole-blood perfusion for $3.5 \mathrm{~min}$ at wall shear rate of $1600 \mathrm{~s}^{-1}$. Numbering of surfaces is as for Figure 1 . First rows: phase-contrast images $(115 \times 115 \mu \mathrm{m})$ for analysis of morphological score, integrated feature size and platelet deposition (surface area coverage). Second and third rows: confocal fluorescence images $(200 \times 200 \mu \mathrm{m})$ captured after staining with FITC-labeled anti-fibrinogen mAb (fibrinogen binding) or FITC-labeled anti-CD62P mAb (P-selectin expression). Fourth rows: single plane confocal images $(106 \times 106 \mu \mathrm{m})$ of DiOC 6 -labeled platelets, used for determination of thrombus volume. Fifth rows: confocal fluorescence images $(200 \times 200 \mu \mathrm{m})$ after staining with AF647-annexin A5 (procoagulant activity). 
A

Cluster analysis (8 parameters)

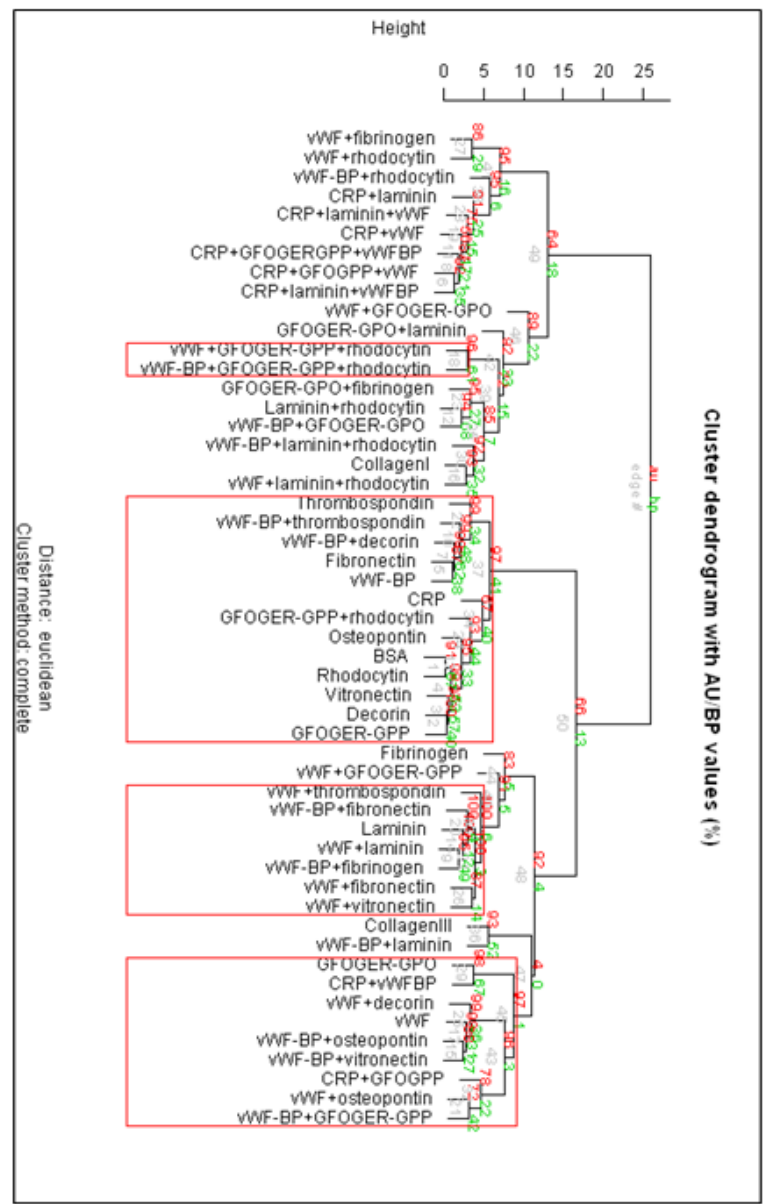

B

Cluster analysis (6 parameters)

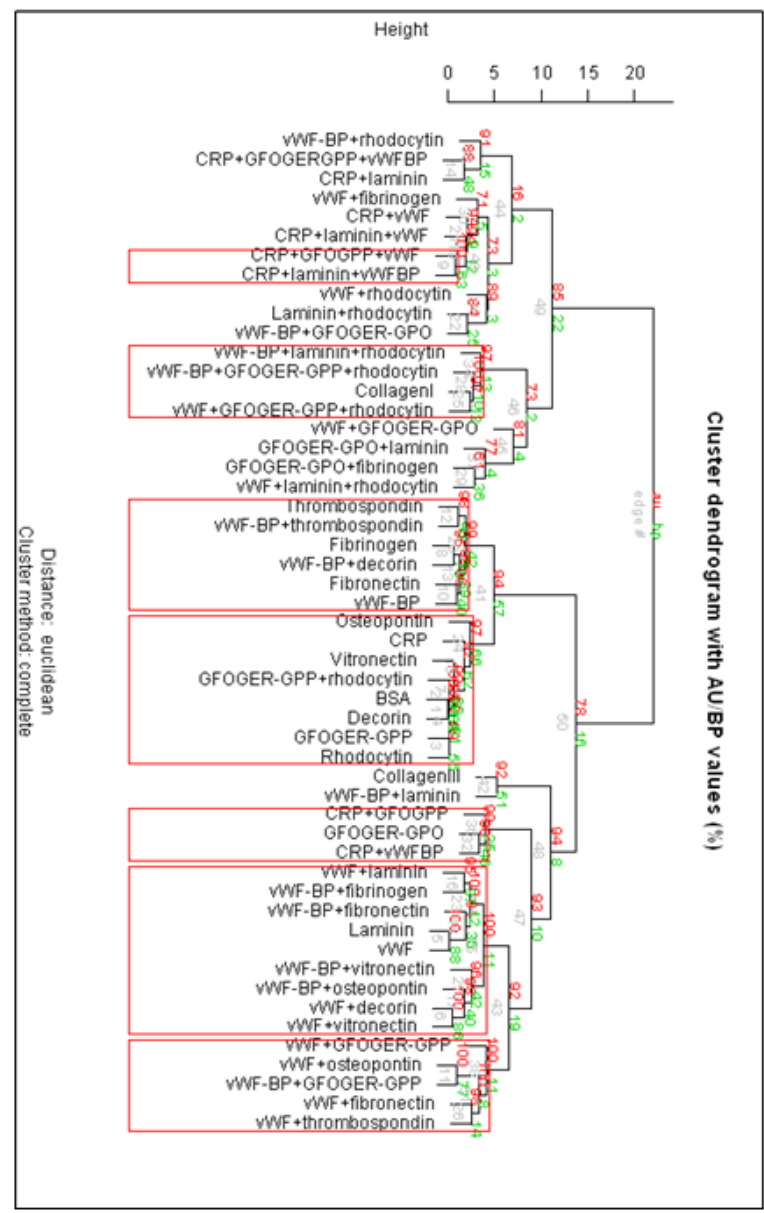

Supplemental Figure 3. Robustness of clustering of surfaces to determine thrombus type. (A) Unsupervised hierarchical clustering of data from 52 surfaces and 8 measurement parameters, as for Figure 3. (B) Similar clustering pattern after removal of two parameters (stable platelet adhesion and thrombus volume), using complete linkage analysis by Pvclust. Note the similar division into type I-III thrombi. Red boxes indicate corresponding grouping of surfaces for either cluster analysis. 


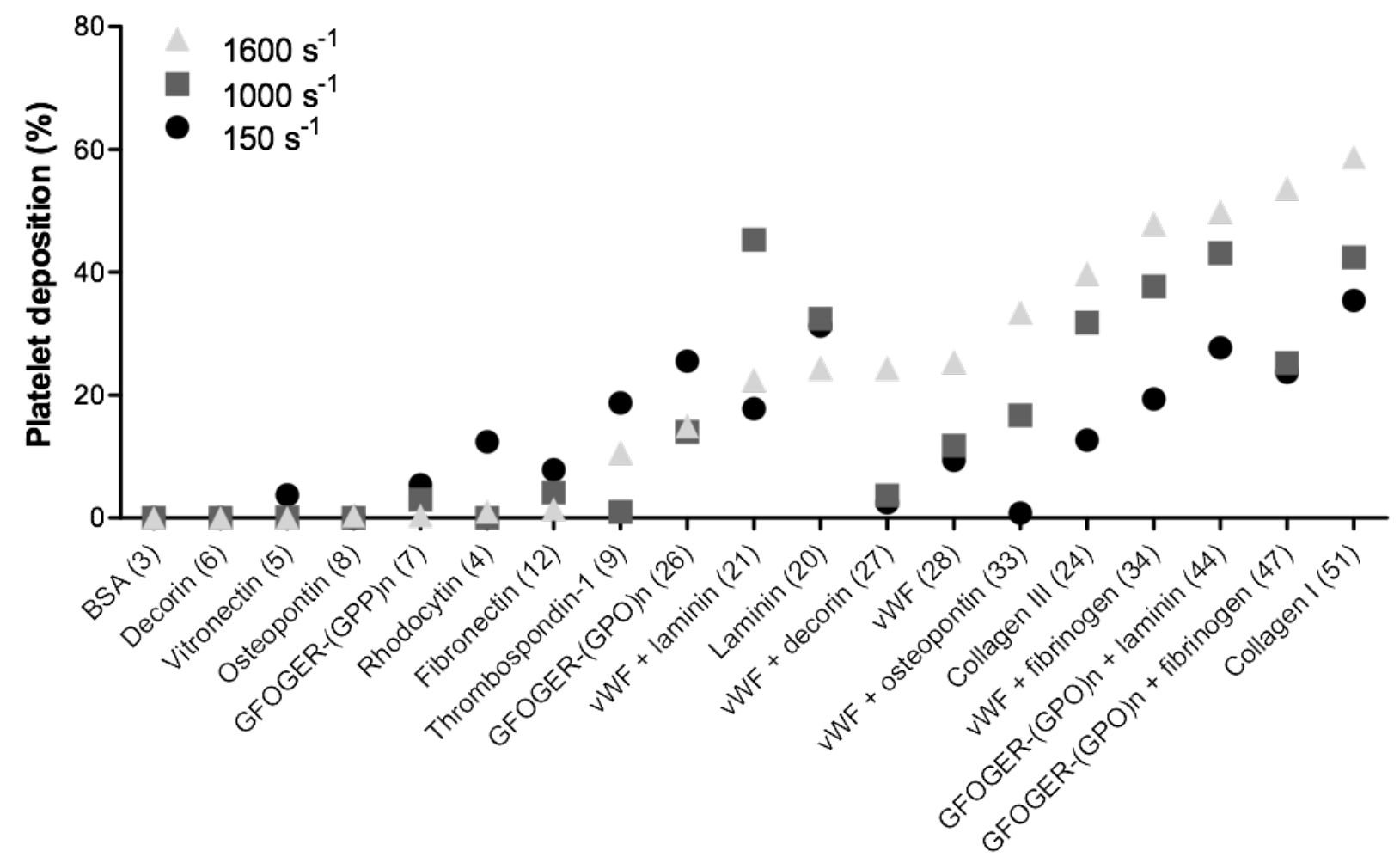

Supplemental Figure 4. Effect of increasing wall shear rate on surface area coverage by platelets. Whole blood was perfused over 19 microspot surfaces at wall shear rates of $150 \mathrm{~s}^{-1}, 1000 \mathrm{~s}^{-1}$ or $1600 \mathrm{~s}^{-1}$. Phase-contrast images were analyzed for surface area coverage with platelets. Ranking from low to high according to change between $150 \mathrm{~s}^{-1}$ and $1600 \mathrm{~s}^{-1}$. 
Supplemental Table 1. Eexpression levels of platelet-adhesive receptors and protein/peptide ligands binding to these receptors. Only ligands used in this study are indicated. Synthetic peptide ligands are shown in italic.

\begin{tabular}{llll} 
Receptor & Protein/peptide ligand & Copies per platelet & Ref(s) \\
\hline $\mathrm{a}_{\mathrm{IIb}} \beta_{3}$ & fibrinogen, fibronectin, vitronectin & $80,000-100,000$ & $1,2,3,4$ \\
GPIb-V-IX & VWF, VWF-BP* & $30,000-40,000$ & 5,6 \\
CD36 & thrombospondin-1 & $10,000-25,000$ & 7 \\
$\mathrm{a}_{2} \beta_{1}$ & collagen I*, III*, decorin, GFOGER & $2,000-4,000$ & $8,9,10,11,12$ \\
GPVI & collagen I, III, (GPO) & $1,000-2,000$ & $10,11,12$ \\
CLEC-2 & podoplanin, rhodocytin & 1,500 & $13,14,15$ \\
$\mathrm{a}_{5} \beta_{1}$ & fibronectin & 1,000 & 3 \\
$\mathrm{a}_{6} \beta_{1}$ & laminin* & 1,000 & 16,17 \\
$\mathrm{a}_{\mathrm{v}} \beta_{3}$ & vitronectin, osteopontin & 500 & 18,19 \\
\hline
\end{tabular}

*Binds VWF from plasma 5, 20,21 
Supplemental Table 2. Prediction of type of thrombus formation on microspot surfaces at low shear flow conditions. Given are per surface the calculated mean parameter values for high shear rate $\left(1600 \mathrm{~s}^{-1}\right)$ and low shear rate $\left(150 \mathrm{~s}^{-1}\right)$. Also shown are the predictive values of mean parameters and thrombus type, obtained through model building using partial least-squares regression analysis of the high-shear data, and fitting into the model the low-shear data (UV scaled). Calculated and predicted thrombus types I, II and III are indicated in gray.

\begin{tabular}{|c|c|c|c|c|c|}
\hline \multirow[t]{2}{*}{ No. } & \multirow[t]{2}{*}{ Surface } & \multicolumn{4}{|c|}{ Mean parameter values (thrombus type) } \\
\hline & & $\begin{array}{l}\text { calculated } \\
1600 \mathrm{~s}^{-1}\end{array}$ & $\begin{array}{l}\text { calculated } \\
150 \mathrm{~s}^{-1}\end{array}$ & $\begin{array}{l}\text { predicted } \\
150 \mathrm{~s}^{-1}\end{array}$ & $\begin{array}{l}\text { type difference } \\
\text { VS. } 1600 \mathrm{~s}^{-1}\end{array}$ \\
\hline 1 & $(\mathrm{GPO})_{\mathrm{n}}$ & $0.46(\mathrm{I})$ & 0.44 & $1.09(\mathrm{I})$ & \\
\hline 2 & GFOGER-(GPP) $)_{n}+$ rhodocytin & $0.02(\mathrm{I})$ & 3.29 & 2.31 (II) & +1 \\
\hline 3 & BSA & $0.10(\mathrm{I})$ & 0.00 & $0.84(\mathrm{I})$ & \\
\hline 4 & Rhodocytin & $0.04(\mathrm{I})$ & 2.40 & $1.92(\mathrm{II})$ & +1 \\
\hline 5 & Vitronectin & $0.10(\mathrm{I})$ & 1.01 & $1.41(\mathrm{I})$ & \\
\hline 6 & Decorin & $0.01(\mathrm{I})$ & 0.00 & $0.84(\mathrm{I})$ & \\
\hline 7 & GFOGER-(GPP) ${ }_{n}$ & $0.05(\mathrm{I})$ & 1.78 & $1.80(\mathrm{II})$ & +1 \\
\hline 8 & Osteopontin & $0.57(\mathrm{I})$ & 0.00 & $0.84(\mathrm{I})$ & \\
\hline 9 & Thrombospondin-1 & $1.50(\mathrm{I})$ & 1.93 & 1.97 (II) & +1 \\
\hline 12 & Fibronectin & $1.06(\mathrm{I})$ & 1.23 & $1.55(\mathrm{II})$ & +1 \\
\hline 14 & Fibrinogen & $1.07(\mathrm{I})$ & 2.52 & $2.05(\mathrm{II})$ & +1 \\
\hline 15 & vWF + GFOGER-(GPP) ${ }_{n}^{*}$ & $3.53(\mathrm{II})$ & 1.66 & $1.62(\mathrm{II})$ & \\
\hline 16 & vWF + thrombospondin-1 & 3.33 (II) & 2.26 & 2.05 (II) & \\
\hline 17 & vWF + vitronectin ${ }^{*}$ & $2.71(\mathrm{II})$ & 2.08 & $1.91(\mathrm{II})$ & \\
\hline 18 & vWF + fibronectin ${ }^{*}$ & $3.43(\mathrm{II})$ & 1.71 & $1.73(\mathrm{II})$ & \\
\hline 20 & Laminin & $2.21(\mathrm{II})$ & 2.58 & 2.31 (II) & \\
\hline 21 & vWF + laminin* & $2.37(\mathrm{II})$ & 1.86 & $1.81(\mathrm{II})$ & \\
\hline 24 & Collagen III (vWF) & $4.96(\mathrm{II})$ & 2.59 & $2.13(\mathrm{II})$ & \\
\hline 26 & GFOGER-(GPO) $)_{n}$ & 4.04 (II) & 4.53 & $2.92(\mathrm{III})$ & +1 \\
\hline 27 & vWF + decorin & $2.85(\mathrm{II})$ & 2.81 & $1.20(\mathrm{I})$ & -1 \\
\hline 28 & VWF $^{*}$ & 2.22 (II) & 1.30 & $1.56(\mathrm{II})$ & \\
\hline 31 & $(G P O)_{n}+$ GFOGER-(GPP) $)_{n}$ & $4.64(\mathrm{II})$ & 3.69 & $2.74(\mathrm{III})$ & +1 \\
\hline 33 & vWF + osteopontin & 4.13 (II) & 0.70 & $1.17(\mathrm{I})$ & -1 \\
\hline 34 & vWF + fibrinogen ${ }^{* \#}$ & 6.02 (III) & 2.81 & 2.24 (II) & -1 \\
\hline 35 & vWF + rhodocytin* & 6.77 (III) & 3.06 & $2.31(\mathrm{II})$ & -1 \\
\hline 37 & $(\mathrm{GPO})_{\mathrm{n}}+$ laminin & $5.53(\mathrm{III})$ & 3.99 & $2.94(\mathrm{III})$ & \\
\hline 38 & $\mathrm{vWF}+(\mathrm{GPO})_{\mathrm{n}}+$ laminin & $6.76(\mathrm{III})$ & 3.87 & $2.83(\mathrm{III})$ & \\
\hline 39 & $\mathrm{vWF}+(\mathrm{GPO})_{\mathrm{n}}$ & $6.56(\mathrm{III})$ & 4.06 & 2.95 (III) & \\
\hline 40 & $\begin{array}{l}\text { vWF-BP }+(G P O)_{n}+ \\
\text { GFOGER(GPP) }\end{array}$ & $5.97(\mathrm{III})$ & 3.98 & $3.22(\mathrm{III})$ & \\
\hline 42 & $\begin{array}{l}\text { vWF + }(G P O)_{n}+\text { GFOGER- } \\
(G P P)_{n}\end{array}$ & $6.26(\mathrm{III})$ & 4.70 & $3.22(\mathrm{III})$ & \\
\hline 43 & vWF + GFOGER-(GPO) ${ }_{n}^{*}$ & $8.66(\mathrm{III})$ & 4.60 & $2.96(\mathrm{III})$ & \\
\hline 44 & GFOGER-(GPO) $n+$ laminin & $8.30($ III $)$ & 3.99 & 2.69 (III) & \\
\hline 45 & $\begin{array}{l}\text { vWF + GFOGER-(GPP) })_{n}+ \\
\text { rhodocytin }\end{array}$ & 8.08 (III) & 3.95 & $2.88(\mathrm{III})$ & \\
\hline 47 & GFOGER-(GPO) $)_{n}+$ fibrinogen & 7.94 (III) & 3.12 & 2.45 (II) & -1 \\
\hline 48 & Laminin + rhodocytin & 7.19 (III) & 4.22 & 3.00 (III) & \\
\hline 51 & Collagen I (vWF) & 7.95 (III) & 4.92 & 3.25 (III) & \\
\hline 52 & vWF + laminin + rhodocytin & 8.49 (III) & 3.68 & 2.59 (III) & \\
\hline
\end{tabular}




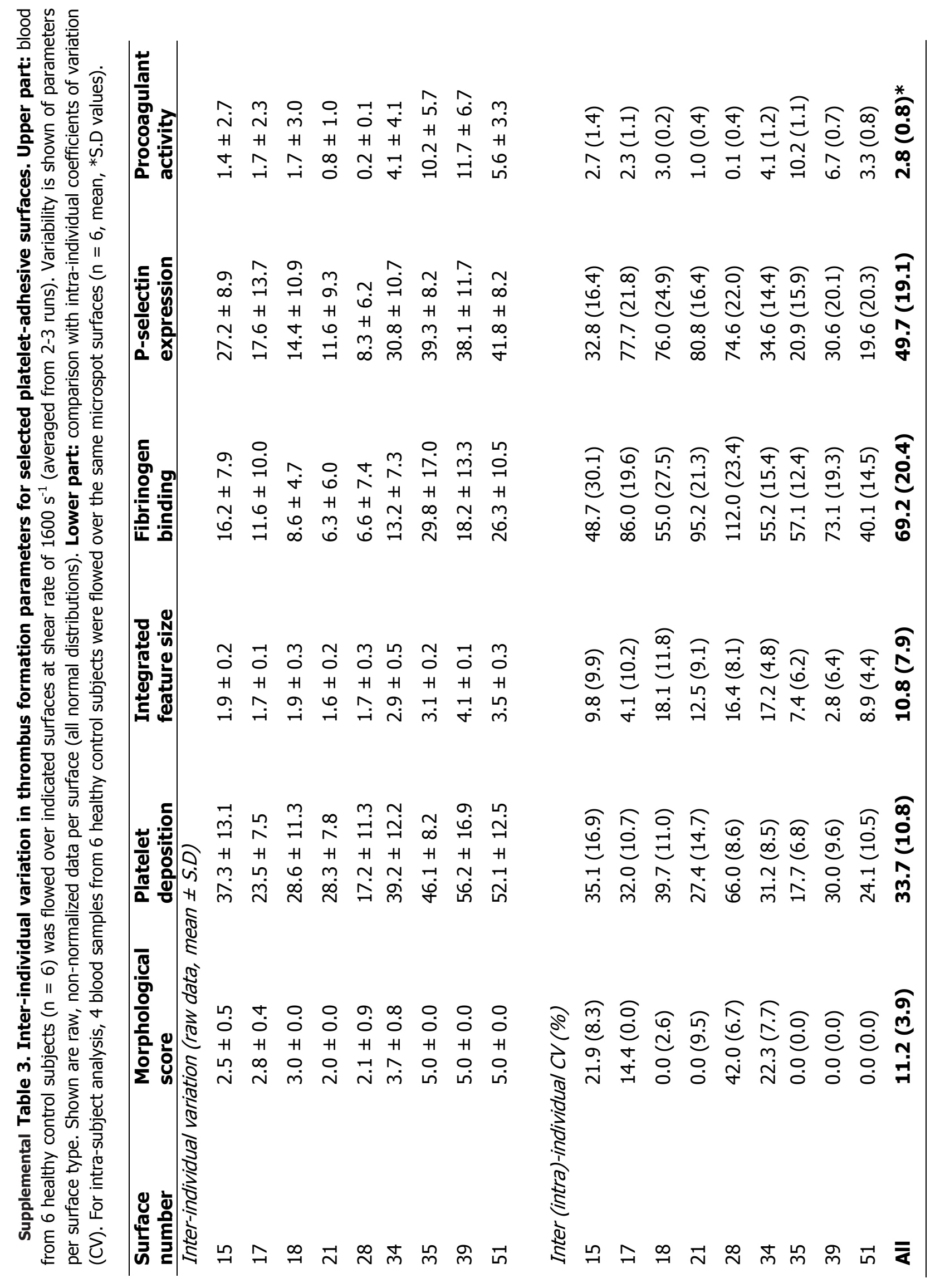


Supplemental Table 4. Effect of thrombin generation on parameters of thrombus formation. Thrombus formation was assayed by 3.5 minutes flow of blood samples at $1600 \mathrm{~s}^{-1}$ over indicated microspot surfaces also containing tissue factor $(0.25 \mathrm{fmol})$. Perfusion was with PPACK/fragmin anticoagulated blood (- thrombin) or with recalcified citrate-anticoagulated blood, containing $5 \mathrm{\mu g} \mathrm{ml}^{-1}$ corn trypsin inhibitor and $5 \mathrm{mg} \mathrm{ml}^{-1} \mathrm{GPRP}$ (+ thrombin). Data are mean \pm S.D ( $n=4$; Mann-Whitney $U$ test). * contraction and increased fluorescent intensity.

\begin{tabular}{lllll}
\hline Coated protein & Parameter & - Thrombin & + Thrombin & P-value \\
\hline vWF/fibronectin & morphological score & $3.0 \pm 0.0$ & $5.0 \pm 0.0$ & 0.008 \\
& integrated feature size & $1.9 \pm 0.4$ & $4.3 \pm 0.3$ & 0.002 \\
& fibrinogen binding & $5.9 \pm 2.7$ & $11.7 \pm 1.1$ & 0.021 \\
& P-selectin expression & $14.6 \pm 9.2$ & $28.6 \pm 2.7$ & 0.027 \\
& platelet deposition & $33.9 \pm 10.8$ & $64.4 \pm 3.5$ & 0.008 \\
& procoagulant activity & $1.0 \pm 1.3$ & $4.4 \pm 3.0$ & 0.049 \\
& & & & \\
Collagen I & morphological score & $5.0 \pm 0.0$ & $5.0 \pm 0.0$ & 1.000 \\
& integrated feature size & $3.5 \pm 0.3$ & $3.5 \pm 0.6$ & 0.519 \\
& fibrinogen binding & $27.1 \pm 10.6$ & $7.7 \pm 1.4^{*}$ & 0.021 \\
& P-selectin expression & $48.2 \pm 7.5$ & $21.4 \pm 2.2^{*}$ & 0.008 \\
& platelet deposition & $58.7 \pm 10.3$ & $40.8 \pm 5.7^{*}$ & 0.042 \\
& procoagulant activity & $4.5 \pm 1.7$ & $7.6 \pm 3.8$ & 0.705 \\
\hline
\end{tabular}


Supplemental Table 5. Origin of proteins and peptides used for coating of microspots.

\begin{tabular}{|c|c|c|c|}
\hline Coated protein & $\begin{array}{l}\text { Coating concentration } \\
(\mu \mathrm{g} / \mathrm{mL})\end{array}$ & Purified from & Company (Ref.) \\
\hline Collagen I (Horm) & 100 & equine tendon & Nycomed (10345787) \\
\hline Collagen III & 100 & human placenta & Sigma Aldrich (C4407) \\
\hline Decorin & 250 & human plasma & Sigma Aldrich ( D8428) \\
\hline Fibrinogen & 250 & human plasma & Sigma Aldrich (F3879) \\
\hline Fibronectin & 250 & human plasma & Sigma Aldrich (F2006) \\
\hline GFOGER-(GPO)n & 250 & chemical synthesis & Ref. ${ }^{12}$ \\
\hline GFOGER-(GPP)n & 250 & chemical synthesis & Ref. ${ }^{12}$ \\
\hline (GPO)n (cross-linked) & 250 & chemical synthesis & Ref. ${ }^{12}$ \\
\hline Laminin $(511 / 521)$ & 100 & human placenta & Sigma Aldrich (L6274) \\
\hline Rhodocytin & 250 & venom Malayan pit viper & Ref. ${ }^{15}$ \\
\hline Thrombospondin-1 & 100 & human platelets & Calbiochem (605225) \\
\hline Osteopontin & 50 & recombinant (human) & R\&D Systems (1433-OP-050 \\
\hline Vitronectin & 50 & human plasma & Sigma Aldrich (V8379) \\
\hline vWF & 50 & human plasma & Ref. $^{6}$ \\
\hline vWF-BP & 100 & chemical synthesis & Ref. ${ }^{5}$ \\
\hline
\end{tabular}

\section{Supplemental references}

1. Savage $B$, Bottini $E$, Ruggeri ZM. Interaction of integrin $\alpha_{\mathrm{IIb}} \beta_{3}$ with multiple fibrinogen domains during platelet adhesion. J Biol Chem 270, $28812-28817$ (1995).

2. Savage B, Saldivar E, Ruggeri ZM. Initiation of platelet adhesion by arrest onto fibrinogen or translocation on von Willebrand factor. Cell 84, 289-297 (1996).

3. Chada $D$, Mather T, Nollert MU. The synergy site of fibronectin is required for strong interaction with the platelet integrin $\alpha_{\mathrm{It}} \beta_{3}$. Ann Biomed Engin 34, 1542-1552 (2006).

4. Zaidi TN, McIntire LV, Farrell DH, Thiagarajan, P. Adhesion of platelets to surface-bound fibrinogen under flow. Blood 88, 2967-2972 (1996).

5. Ruggeri ZM, Mendolicchio GL. Adhesion mechanisms in platelet function. Circ Res 100, 1673-1685 (2007).

6. Pugh N, Simpson AM, Smethurst PA, de Groot PG, Raynal N, Farndale RW. Synergism between platelet collagen receptors defined using receptor-specific collagen-mimetic peptide substrata in flowing blood. Blood 115, 5069-5079 (2010).

7. Nergiz-Unal R, Lamers MM, Van Kruchten R, Luiken JJ, Cosemans JM, Glatz JF, Kuijpers MJ, Heemskerk JW. Signaling role of CD36 in platelet activation and thrombus formation on immobilized thrombospondin or oxidized low-density lipoprotein. J Thromb Haemost 9, 1835-1846 (2011).

8. Fiedler LR, Schönherr E, Waddington R, Niland S, Seidler DG, Aeschlimann D, Eble JA. Decorin regulates endothelial cell motility on collagen I through activation of insulin-like growth factor I receptor and modulation of $\alpha_{2} \beta_{1}$ integrin activity. J Biol Chem 283, 17406-17415 (2008).

9. Inoue $\mathrm{O}$, Suzuki-Inoue $\mathrm{K}$, Dean WL, Frampton J, Watson SP. Integrin $\alpha_{2} \beta_{1}$ mediates outside-in regulation of platelet spreading on collagen through activation of Src kinases and PLCY2. J Cell Biol 160, 769-780 (2003).

10. Munnix IC, Gilio K, Siljander PR, Raynal N, Feijge MA, Hackeng TM, Deckmyn H, Smethurst PA, Farndale RW, Heemskerk JW. Collagen-mimetic peptides mediate flow-dependent thrombus formation by high- or low-affinity binding of integrin $\alpha_{2} \beta_{1}$ and glycoprotein VI. J Thromb Haemost 6, 2132-2142 (2008). 
11. Siljander PR, Munnix IC, Smethurst PA, Deckmyn H, Lindhout T, Ouwehand WH, Farndale RW, Heemskerk JW. Platelet receptor interplay regulates collagen-induced thrombus formation in flowing human blood. Blood 103, 1333-1341 (2004).

12. Smethurst $P A$, Joutsi-Korhonen $L$, O'Connor MN, Wislon $E$, Jennings NS, Garner SF, Zhang $Y$, Knight CG, Dafforn TR, Buckle A, IJsseldijk MJ, De Groot PG, Watkins NA, Farndale RW, Ouwehand WH. Identification of the primary collagen-binding surface on human glycoprotein VI by site-directed mutagenesis and by a blocking phage antibody. Blood 103, 903-911 (2004).

13. Bergmeier W, Bouvard D, Eble JA, Mokhtari-Nejad R, Schulte V, Zirngible H, Brakebusch C, Fassler $\mathrm{R}$, Nieswandt B. Rhodocytin (aggretin) activates platelets lacking a2 $\beta 1$ integrin, glycoprotein VI, and the ligand-binding domain of glycoprotein Iba. J Biol Chem 276, 25121-25126 (2001).

14. Watson AA, Cristou CM, James JR, Fenton-May AE, Moncayo GE, Mistry AR, Davis SJ, Gilbert RJ, Chakera A, O'Callaghan CA. The platelet receptor CLEC-2 is active as a dimer. Biochemistry 48, 1098810996 (2009).

15. Hooley E, Papagrigoriou E, Navdaev A, Panday AV, Clemetson JM, Clemetson KJ, Emsley J. The crystal structure of the platelet activator aggretin reveals a novel $(\alpha \beta)_{2}$ dimeric structure. Biochemistry 47, 7831-7837 (2008).

16. Inoue O, Suzuki-Inoue K, McCarty O, Moroi M, Ruggeri ZM, Kunicki TJ, Ozaki Y, Watson SP. Laminin stimulates spreading of platelets through integrin a6ß1-dependent activation of GPVI. Blood 107, 1405-1412 (2006).

17. Schaff M, Tang C, Maurer E, Bourdon C, Receveur N, Eckly A, Hechler B, Arnold C, de Arcangelis A, Nieswandt B, Denis CV, Lefebvre O, Georges-Labouesse E, Gachet C, Lanza F, Mangin PH. Integrin $\alpha 6 \beta 1$ is the main receptor for vascular laminins and plays a role in platelet adhesion, activation and arterial thrombosis. Circulation 128, 541-552 (2013).

18. Asch E \& Podack E. Vitronectin binds to activated human platelets and plays a role in platelet aggregation. J Clin Invest 85, 1372-1378 (1990).

19. Reheman A, Gross P, Yang H, Chen P, Allen D, Leytin V, Freedman J, Ni H. Vitronectin stabilizes thrombi and vessel occlusion but plays a dual role in platelet aggregation. J Thromb Haemost 3, 875-883 (2005).

20. Pareti FI, Niiya K, McPherson JM, Ruggeri ZM. Isolation and characterization of two domains of human von Willebrand factor that interact with fibrillar collagen types I and III. J Biol Chem 262, 1383513841 (1987).

21. Roth GJ, Titani K, Hoyer LW, Hickey MJ. Localization of binding sites within human von Willebrand factor for monomeric type III collagen. Biochemistry 25, 8357-8361 (1986). 
A

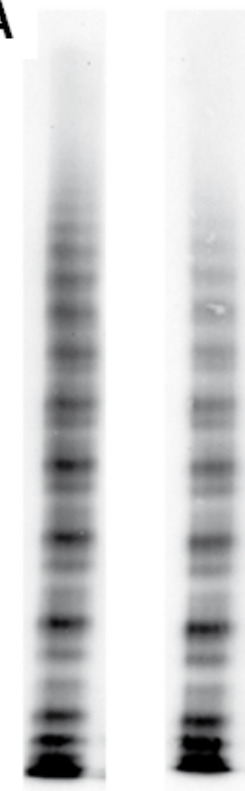

original sample
B
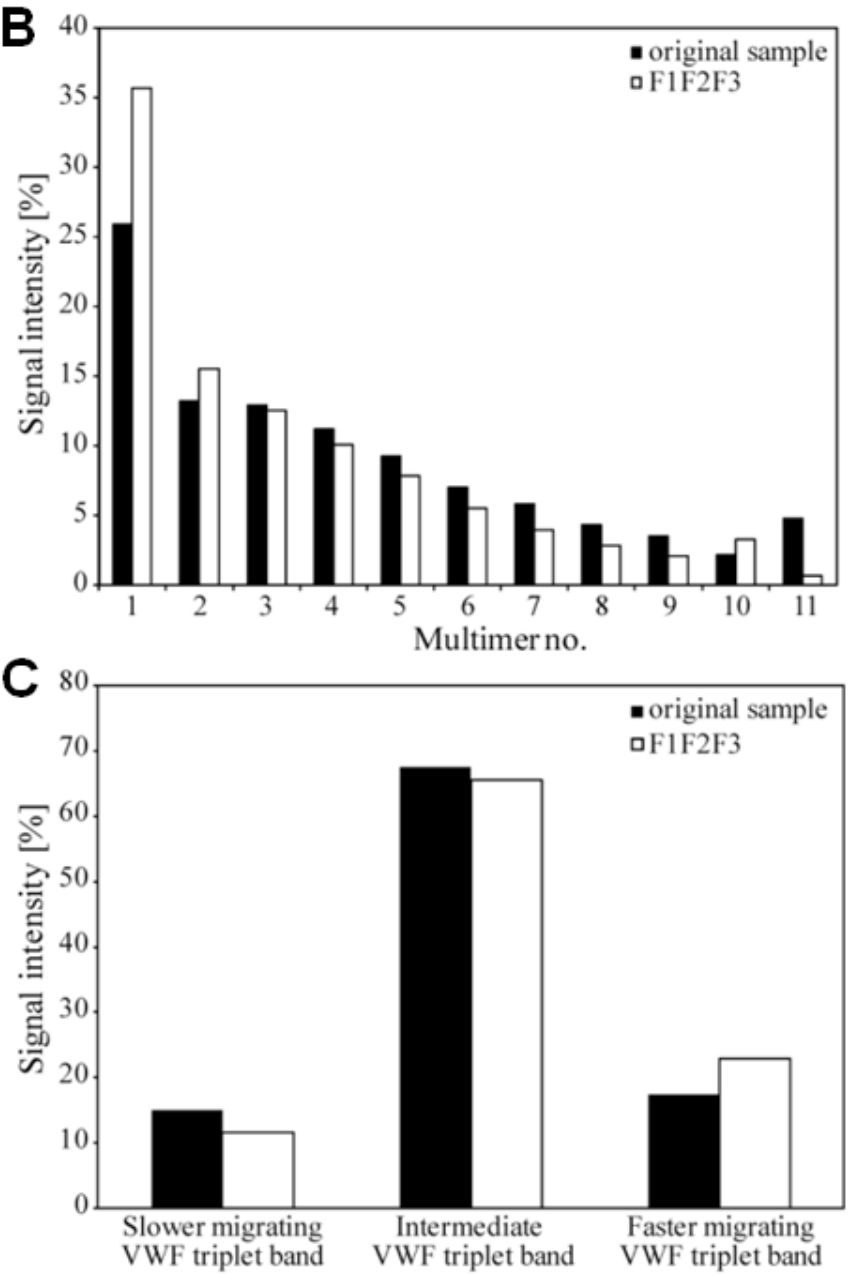

Supplemental Figure 1. Multimeric pattern and triplet structure analyses of original VWF concentrate sample (Wilate) and recombined fractions F1F2F3 after heparin affinity chromatography. (A) Multimeric pattern of original sample and recombined fractions F1F2F3, separated on 1.2\% agarose gel. (B) Composition of multimers, as obtained by densitometric analysis of Western blots. (C) Proportions of each of the three triplet bands in the original sample and F1F2F3. Values of VWF:RCo activity, as fractions of antigen levels, were determined as $0.41 \pm 0.04$ (original sample) and $0.35 \pm 0.01$ (F1F2F3). Means \pm SEM $(n=3)$. 

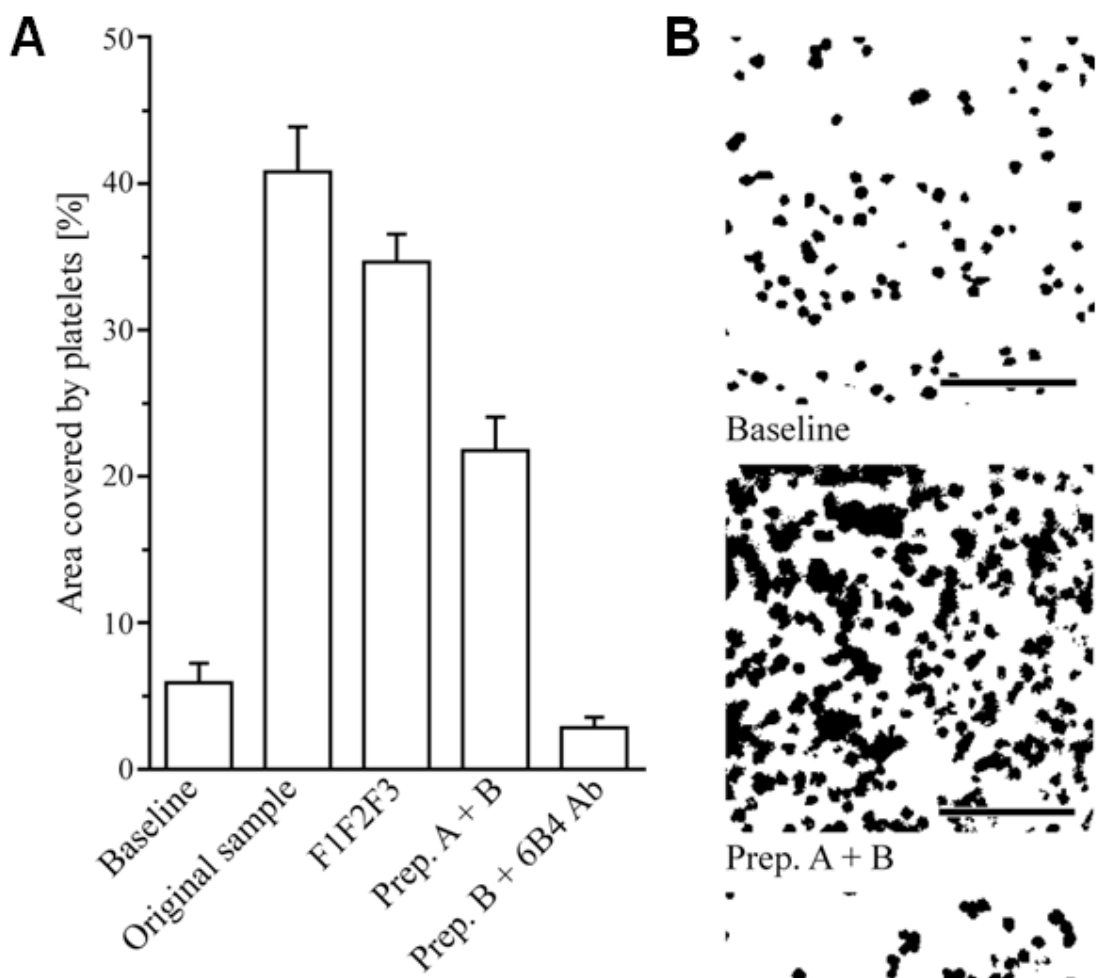

Baseline

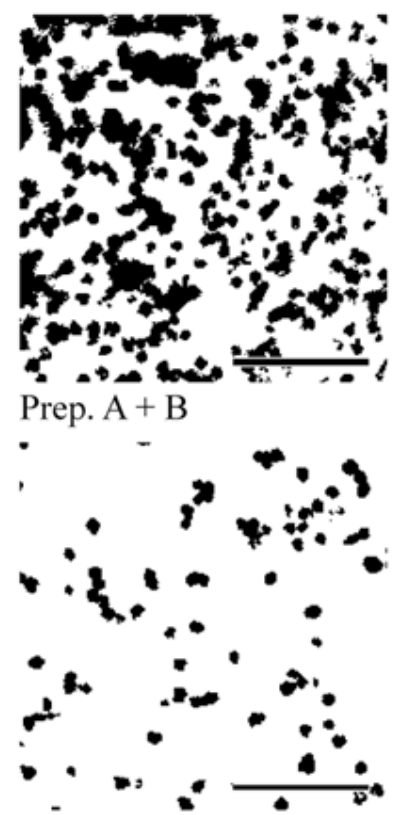

Prep. $\mathrm{B}+6 \mathrm{~B} 4 \mathrm{Ab}$

Supplemental Figure 2. Flow chamber experiments performed with reconstituted blood containing CMFDA-labelled platelets $\left(2.5 \times 10^{8} / \mathrm{mL}\right)$ and washed red blood cells (40\% hematocrit), flowed over a type III collagen surface. The reconstituted blood samples were supplemented with various VWF preparations (2 IU/mL, final), i.e. original VWF concentrate sample, the combined fractions F1F2F3, or equal amounts of preparations A plus B. The 6B4 mAb $(20 \mu \mathrm{g} / \mathrm{mL})$ against the VWF binding site of GPIb was added, as indicated. Samples without VWF (baseline) served as negative control. (A) Analyzed surface area coverage of fluorescence after 8 min perfusion at $1700 \mathrm{~s}^{-1}$ shear rate. (B) Representative fluorescence images (scale bars; $50 \mu \mathrm{m})$. Means $\pm \operatorname{SEM}(n=2-3)$. 
A

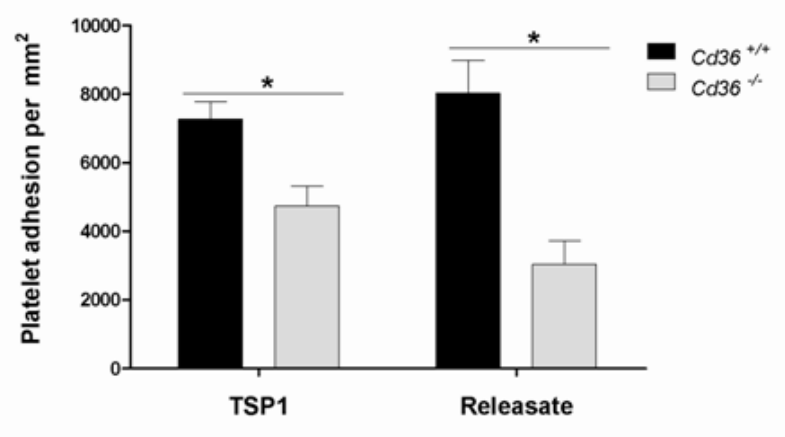

C

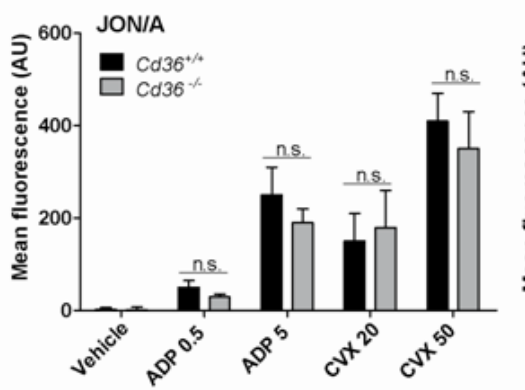

D

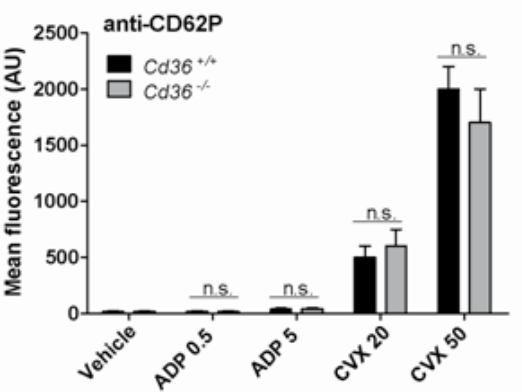

B

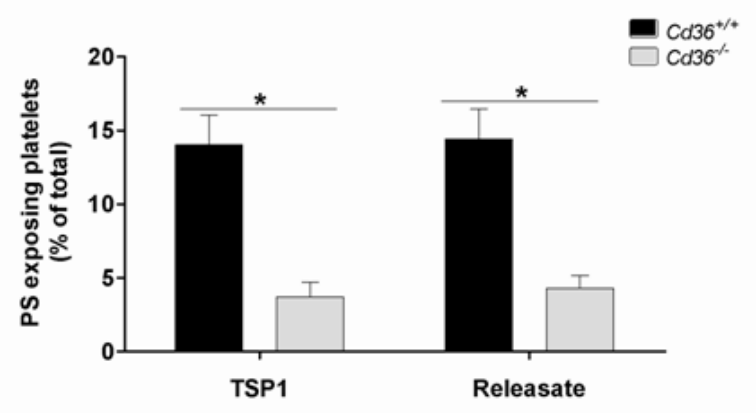

E

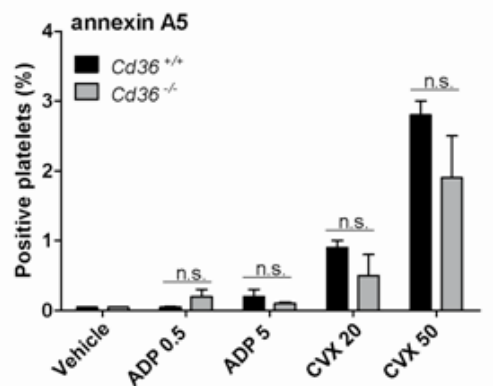

Supplemental Figure 1. Roles of murine CD36 in platelet adhesion to TSP1 and in agonist-induced platelet activation. (A-B) Adhesion of washed platelets from $\mathrm{Cd} 36^{+/+}$or $\mathrm{Cd} 36^{-/}$mice $\left(1.5 \times 10^{8} / \mathrm{mL}\right)$ to surfaces coated with purified TSP1 or with releasate from activated wild type platelets. Platelet adhesion and activation were measured by phase-contrast and fluorescence microscopy after 45 minutes. (A) quantification of adhered platelets (platelets $/ \mathrm{mm}^{2}$ ). (B) quantification of FITC-annexin A5 positive platelets (\% total). (C-E) Washed platelets from $\mathrm{Cd} 36^{+/+}$and $\mathrm{Cd} 36^{-/}$mice were stimulated with ADP $(0.5-5 \mu \mathrm{mol} / \mathrm{L})$ or convulxin $(20-50 \mathrm{ng} / \mathrm{mL})$ during 10 minutes, and analyzed by flow cytometry. (C) Activation of $\alpha_{\mathrm{II}} \beta_{3}$ detected with PE-labeled JON/A mAb, (D) expression of P-Selectin detected with FITC-antiCD62P mAb, and (E) expression of phosphatidylserine detected with FITC-annexin A5. Means \pm SEM $(n=4-5) . * P<0.05$ vs. $\mathrm{Cd} 36^{+/+}$; n.s., not significant. 
A
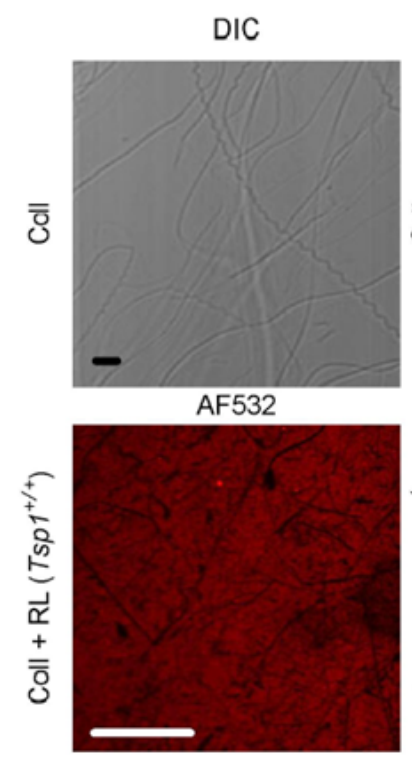

$\overline{\mathrm{o}}$

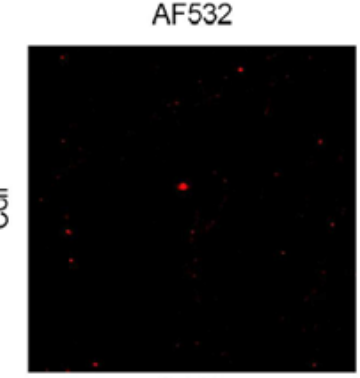

AF532

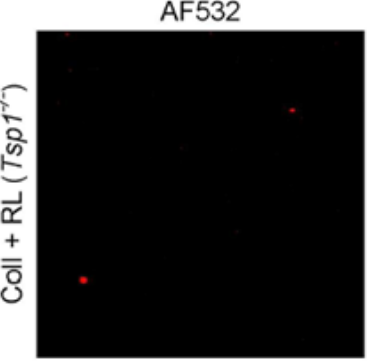

B

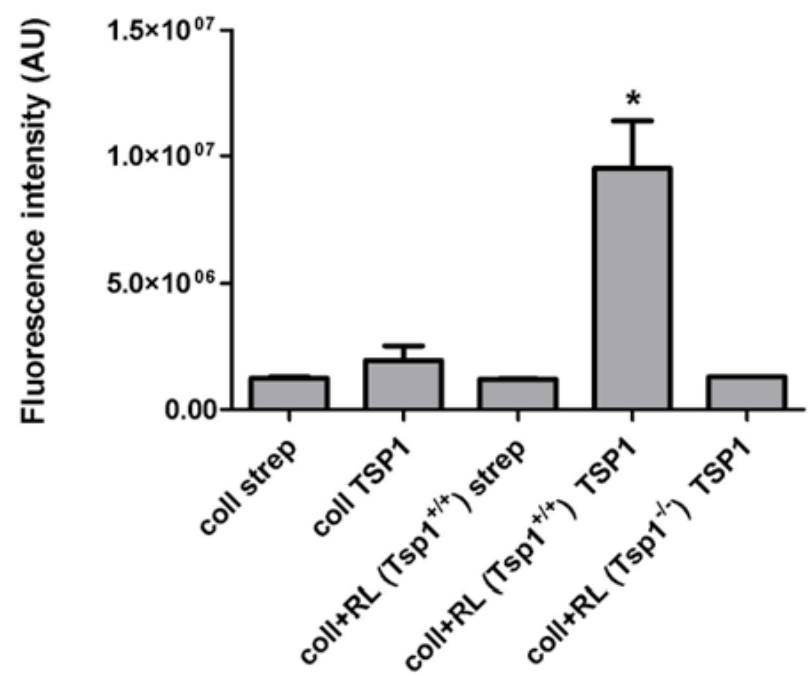

Supplemental Figure 2. Detection of immobilized TSP1 in platelet releasate. Collagen-containing coverslips were post-incubated with releasate $(\mathrm{RL})$ from thrombin-stimulated $T s p 1^{+/+}$or $T s p 1^{\%}$ platelets, as indicated. Surfaces were stained with biotin-conjugated anti-TSP1 antibody and AF532-streptavidin. (A) Representative images of differential interference contrast(DIC) and confocal AF532-streptavidin fluorescence (AF532); bars, $25 \mu \mathrm{m}$. (B) Integrated fluorescence intensity from coated surfaces (arbitrary units). Strep indicates control staining with only AF532-streptavidin; TSP1 indicates staining with anti-TSP1 antibody and AF532-streptavidin. Means $\pm \operatorname{SEM}(n=3)$.
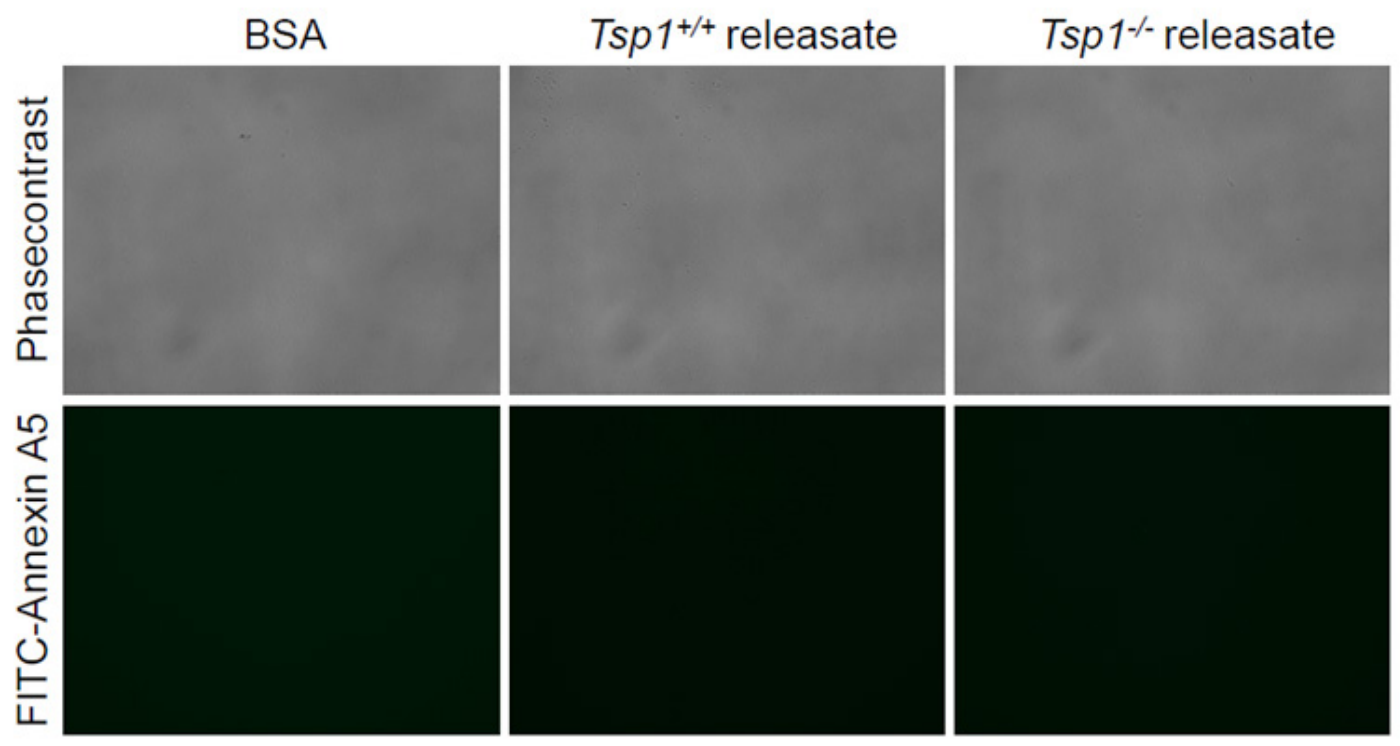

Supplemental Figure 3. Absence of phosphatidylserine-exposing microparticles in coated releasate from

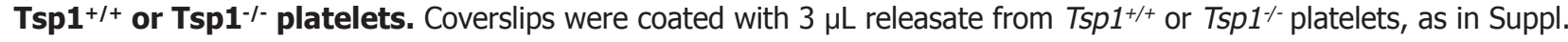
Figure 2. Surfaces then were blocked with $1 \%$ BSA, and stained with $0.5 \mu \mathrm{g} / \mathrm{mL}$ FITC-annexin A5 for 5 minutes. After rinse with Tyrode's Hepes buffer, phase contrast and fluorescence images were captured from the coated surface. Image size is $107 \times 142 \mu \mathrm{m}$.

Supplemental movies of in vitro thrombus formation.

Movies available online : atvb.ahajournals.org/content/34/6/1187/suppl/DC1 


\section{Supplemental Table 1. Spreadsheet of all tabled meta-data from published mouse studies.}

See mouse_genes_thrombosis.xls (online only).

Supplemental Table 2. Correlation analysis of intra-study parameters scored on 3-point or 5-point scale. Panels represent numbers of studies, Kendall's Tau correlation coefficients, and $P$ values. (A) Comparison of mass-dependent sub-parameters in class I studies (bi, platelet adhesion; bii, extent of occlusion; biii, thrombus size, volume or number). (B) Comparison of mass-dependent sub-parameters in class III studies ( $g i$, platelet adhesion; gii, surface-area-coverage; giii, thrombus size, volume or number). (C) Comparison of time-dependent (a), mass-dependent (b), and stability (c) parameters in class I studies. Time-dependent parameters (a/f) indicated time to occlusion or thrombus formation; stability parameters $(c / h)$ referred to number of emboli. (D) Comparison of time-dependent (e), mass-dependent $(f)$, and stability $(g)$ parameters for class III studies.

A
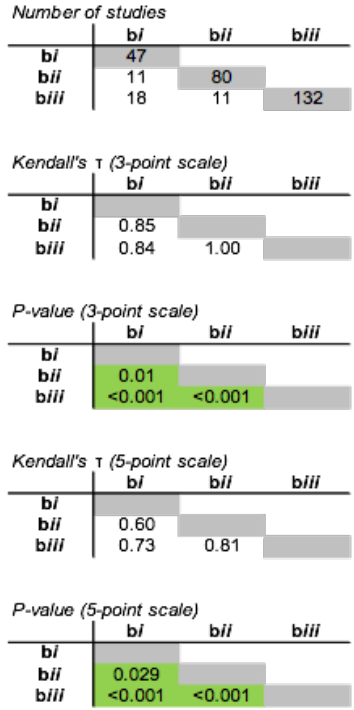

B
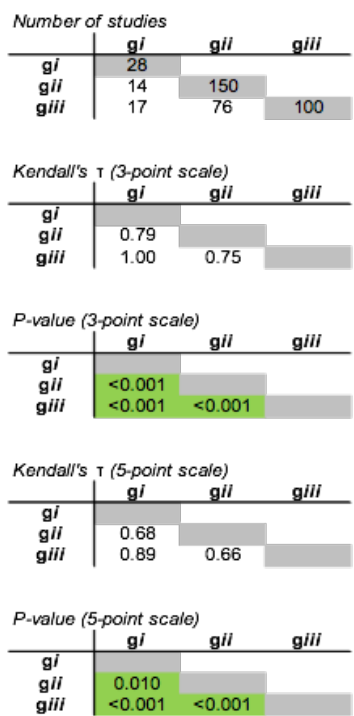

C
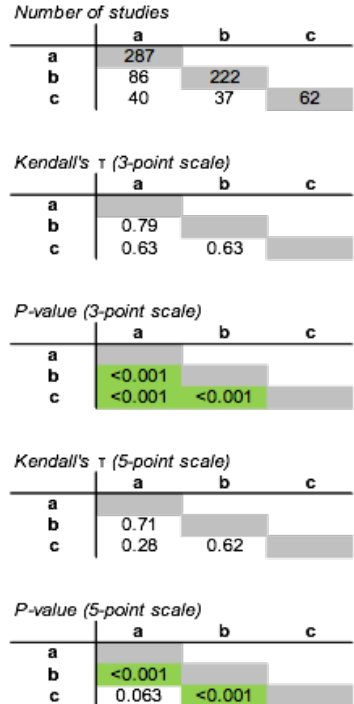
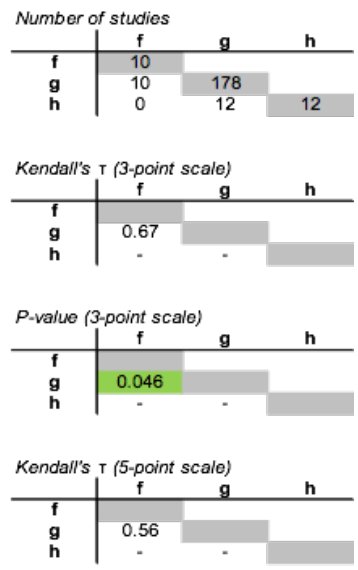

\begin{tabular}{c|ccl}
\multicolumn{2}{c}{$P$-value (5-point scale) } & & \\
& $\mathbf{f}$ & $\mathbf{g}$ & $\mathbf{h}$ \\
\hline $\mathbf{f}$ & & & \\
$\mathbf{g}$ & 0.074 & & \\
$\mathbf{h}$ & - & -
\end{tabular} 
Supplemental Table 3. Correlation analysis of comparative studies per class scored on 3-point or 5-point scale. (A) Comparison of studies in same papers (same mouse strain). (B) Comparison of studies with loss-of-function modification of same gene. Panels represent numbers of compared studies or genes, Kendall's Tau correlation coefficients, and $P$ values (3- or 5-point scores).

A

\begin{tabular}{c|ccc}
\multicolumn{4}{c}{ Number of studies } \\
& I & II & III \\
\hline I & 424 & & \\
II & 0 & 81 & \\
III & 87 & 12 & 178
\end{tabular}

B

\begin{tabular}{c|ccc}
\multicolumn{4}{c}{ Number of genes } \\
& I & II & III \\
\hline I & 194 & & \\
II & 40 & 64 & \\
III & 73 & 24 & 97
\end{tabular}

\begin{tabular}{|c|c|c|c|}
\hline Kendall & T (3-po & t scale) & III \\
\hline$T$ & & & \\
\hline III & 0.75 & 0.85 & \\
\hline
\end{tabular}

\begin{tabular}{|c|c|c|c|}
\hline Kendall's & т (3-poin & t scale) & III \\
\hline T & & & \\
\hline II & 0.83 & & \\
\hline III & 0.74 & 0.78 & \\
\hline
\end{tabular}

\begin{tabular}{|c|c|c|c|}
\hline$P$-value & (3-point sc & & \\
\hline & & II & III \\
\hline III & $<0.001$ & $<0.001$ & \\
\hline
\end{tabular}

\begin{tabular}{|c|c|c|c|}
\hline$P$-value & $(3-p o i n t s c$ & cale) & III \\
\hline$T$ & & & \\
\hline II & $<0.001$ & & \\
\hline III & $<0.001$ & $<0.001$ & \\
\hline
\end{tabular}

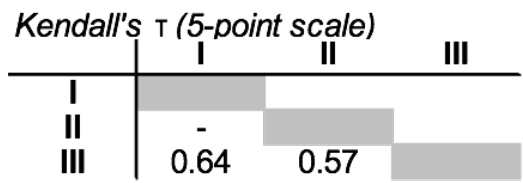

\begin{tabular}{|c|c|c|c|}
\hline Kendall' & т (5-poin & $\underset{\|}{\text { scale) }}$ & III \\
\hline$T$ & & & \\
\hline II & 0.65 & & \\
\hline III & 0.58 & 0.29 & \\
\hline
\end{tabular}

\begin{tabular}{|c|c|c|c|}
\hline$P$-value & 5 -point sc & ale) & \\
\hline & & II & III \\
\hline III & $<0.001$ & 0.012 & \\
\hline
\end{tabular}

\begin{tabular}{|c|c|c|c|}
\hline$P$-value & $\left(\begin{array}{c}5-p o i n t ~ s c e \\
\mid\end{array}\right.$ & le) & III \\
\hline I & & & \\
\hline III & $<0.001$ & 0.12 & \\
\hline
\end{tabular}


Supplemental Table 4. Listing of numbers of genes per class with scores for different groups.

For description of groups, see Table 1.

Class I (number of genes)

\begin{tabular}{|c|c|c|c|c|c|c|c|c|c|c|c|c|c|}
\hline & & 1 & 2 & 3 & 4 & 5 & 6 & 7 & 8 & 9 & 10 & 11 & 12 \\
\hline 1 & Carotis $\mathrm{FeCl}_{3}$ & 112 & & & & & & & & & & & \\
\hline 2 & Carotis photochemical & 11 & 27 & & & & & & & & & & \\
\hline 3 & Carotis ligation & 7 & 2 & 12 & & & & & & & & & \\
\hline 4 & Carotis electrolytical & 3 & 0 & 3 & 4 & & & & & & & & \\
\hline 5 & Aorta compression & 3 & 1 & 0 & 0 & 11 & & & & & & & \\
\hline 6 & Mesentery $\mathrm{FeCl}_{3}$ & 31 & 5 & 6 & 2 & 9 & 67 & & & & & & \\
\hline 7 & Mesentery photochemical & 1 & 1 & 0 & 0 & 0 & 0 & 3 & & & & & \\
\hline 8 & Mesentery laser & 6 & 0 & 1 & 1 & 1 & 5 & 0 & 12 & & & & \\
\hline 9 & Cremaster $\mathrm{FeCl}_{3}$ & 2 & 0 & 1 & 1 & 0 & 4 & 0 & 2 & 4 & & & \\
\hline 10 & Cremaster photochemical & 5 & 0 & 0 & 0 & 0 & 3 & 0 & 0 & 0 & 11 & & \\
\hline 11 & Cremaster laser & 15 & 1 & 4 & 3 & 2 & 14 & 0 & 4 & 4 & 2 & 35 & \\
\hline 12 & Other & 1 & 0 & 2 & 1 & 1 & 3 & 0 & 1 & 1 & 0 & 1 & 7 \\
\hline
\end{tabular}

Class /I (number of genes)

\begin{tabular}{l|l|cccc}
\multicolumn{2}{l|}{} & $\mathbf{2 0}$ & $\mathbf{2 1}$ & $\mathbf{2 2}$ & $\mathbf{2 3}$ \\
\hline $\mathbf{2 0}$ & Injection collagen-epinephrine & 8 & & & \\
$\mathbf{2 1}$ & Injection tissue factor & 2 & 4 & & \\
$\mathbf{2 3}$ & Injection other & 1 & 0 & 1 & \\
& Systemic other & 0 & 1 & 0 & 3
\end{tabular}

Class III (number of genes)

\begin{tabular}{|c|c|c|c|c|c|c|}
\hline & & 30 & 31 & 32 & 33 & 34 \\
\hline 30 & Collagen I & 38 & & & & \\
\hline 31 & Collagen III & 4 & 4 & & & \\
\hline 32 & von Willebrand factor & 1 & 0 & 1 & & \\
\hline 33 & Fibrinogen & 2 & 1 & 0 & 2 & \\
\hline 34 & Other & 0 & 0 & 0 & 0 & 0 \\
\hline
\end{tabular}

Class $N$ (number of genes)

\begin{tabular}{l|l|c}
\multicolumn{2}{l|}{} & $\mathbf{4 0}$ \\
\hline $\mathbf{4 0}$ & Tail bleeding & 266
\end{tabular}


Supplemental Table 5. Overview of scored parameters of thrombus formation(classes I-III) and tail bleeding (class IV) per mouse gene. Also indicated are numbers of studies evaluated $(\mathrm{N})$ and relevant phenotypic effects of modified mice. Abbreviations for tissues affected: A/m, all (mutation); B, blood; $E$, endothelial cells; $H$, hematopoietic cells; L, liver; M, monocytes; P, platelets + megakaryocytes; R, red blood cells; S, smooth muscle cells. For phenotyping: activ., activation; adh., adhesion; aggr., aggregation; CR, clot retraction; GP, glycoprotein; F, factor; MGK, megakaryocyte; RBC, red blood cell; secr., secretion; TG, thrombin generation; WBC, white blood cell. Green and red cells indicated antithrombotic and prothrombotic effects respectively (3- and 5-point scale), yellow is no effect, grey indicates the number of studixss

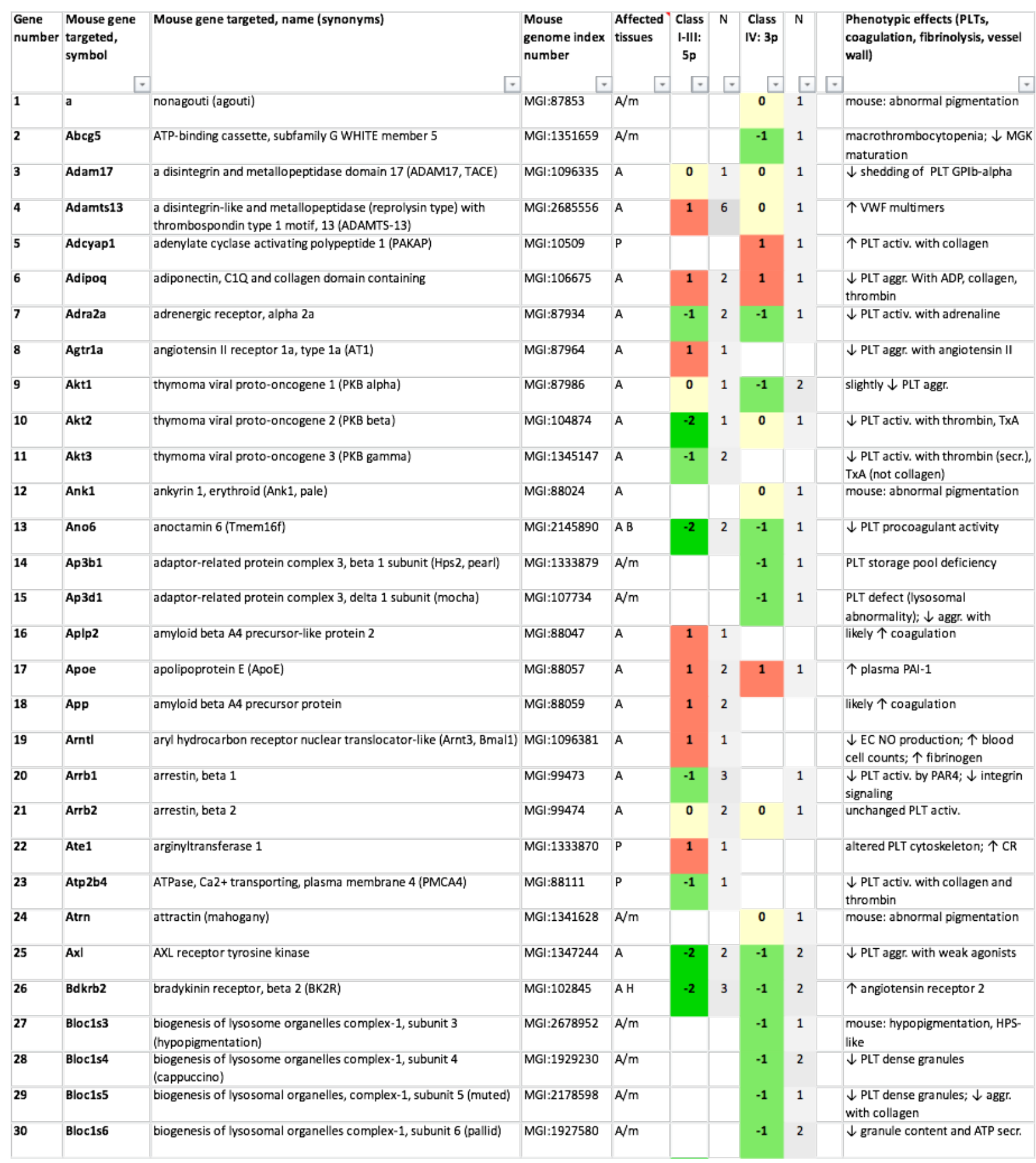




\begin{tabular}{|c|c|c|c|c|c|c|c|c|c|}
\hline 31 & Btk & $\begin{array}{l}\text { Bruton agammaglobulinemia tyrosine kinase (Bruton's tyrosine } \\
\text { kinase) }\end{array}$ & MGI:88216 & A & -2 & 1 & & & $\downarrow$ PLT activ. via GPIb \\
\hline 32 & C1gatt1c1 & C1GALT1-specific chaperone 1 & MGI:1913493 & EH & -2 & 1 & -1 & 1 & $\begin{array}{l}\text { altered 0-glycosylation, } \\
\text { macrothrombocytopenia }\end{array}$ \\
\hline 33 & $\mathrm{C2}(\mathrm{Cfb})$ & complement component $2+$ complement factor $\mathrm{B}$ & MGI:88226 & A & 0 & 1 & & & $\downarrow$ complement activ. \\
\hline 34 & C3 & complement component 3 & MGI:88227 & A & -2 & 1 & -1 & 2 & $\downarrow$ PLT activ. with PAR peptide \\
\hline 35 & C6 & complement component 6 & MGI:88233 & $\mathrm{A} / \mathrm{m}$ & & & -1 & 1 & $\downarrow$ PLT aggr. with ADP \\
\hline 36 & Capn1 & calpain 1 & MG1:88263 & A & -1 & 1 & 0 & 1 & $\begin{array}{l}\downarrow P L T \text { activ. with thrombin, TXA; } \\
\downarrow C R\end{array}$ \\
\hline 37 & cblb & Casitas B-lineage lymphoma $\mathrm{b}|\mathrm{Cb}|-\mathrm{b})$ & MGI:2146430 & A & -2 & 1 & & & $\begin{array}{l}\downarrow \text { PLT activ. with collagen [via } \\
\text { Syk] }\end{array}$ \\
\hline 38 & Cbs & cystathionine beta-synthase & MG1:88285 & A & 0 & 1 & & & $\uparrow$ plasma homocysteine \\
\hline 39 & Cd151 & CD151 antigen [Tspan24] & MGI:1096360 & A & -2 & 3 & -1 & 1 & $\begin{array}{l}\downarrow \text { PLT binding, activ. with laminin, } \\
\text { fibrinogen }\end{array}$ \\
\hline 40 & Cd18 & CD18 antigen (integrin beta 2 , Itgb 2 ) & MGI:96611 & A & -1 & 1 & & & $\downarrow$ WBC-EC adh. \\
\hline 41 & Cd36 & CD36 antigen & MGI:107899 & A & -1 & 7 & 0 & 3 & $\begin{array}{l}\downarrow \text { PLT responses with ox-LDL, } \\
\text { thrombospondin }\end{array}$ \\
\hline 42 & Cd38 & CD38 antigen (CADP-ribose hydrolase) & MGI:107474 & A & -2 & 1 & -1 & 1 & $\begin{array}{l}\downarrow \text { CADP n bose and NAMDP } \\
\text { formation in PLTs }\end{array}$ \\
\hline 43 & Cd 40 & CD 40 antigen & MG1:88336 & A & -1 & 3 & -1 & 1 & $\begin{array}{l}\downarrow \text { signaling in WBC; } \downarrow \text { PLT } \\
\text { binding to WBC }\end{array}$ \\
\hline 44 & Cd40lg & CD40 ligand (CD154) & MG1:88337 & A & -1 & 5 & -1 & 2 & $\begin{array}{l}\downarrow \text { PLT integrin activ.; } \downarrow \text { WBC } \\
\text { function }\end{array}$ \\
\hline 45 & Cdc42 & cell division cycle 42 (Cdc 42$)$ & MGI:106211 & AP & 1 & 2 & -1 & 1 & $\uparrow / \downarrow$ PLT activ. with collagen \\
\hline 46 & Cdh23 & cadherin 23 (waltzer) & MSI:1890219 & $\mathrm{A} / \mathrm{m}$ & & & 0 & 1 & mouse: deafness, altered skin \\
\hline 47 & Ceacam1 & $\begin{array}{l}\text { carcinoembryonic antigen-related cell adhesion molecule } \\
\text { [CEACAM-1] }\end{array}$ & MGI:1347245 & A & 1 & 3 & & & $\uparrow P L T$ activ. with collagen \\
\hline 48 & $\mathrm{Cfb}(\mathrm{Cz})$ & complement factor $\mathrm{B}+$ complement component 2 & MGI:105975 & A & 0 & 1 & & & $\downarrow$ complement activ. \\
\hline 49 & Clb1 & $\begin{array}{l}\text { C-type lectin domain family } 1 \text {, member b (cakium and integrin- } \\
\text { binding 1, CIB1) }\end{array}$ & MGI:1344418 & A & -1 & 2 & -1 & 2 & $\begin{array}{l}\text { normal PLT aggr, integrin activ.; } \downarrow \\
\text { PLT spreading on fibrinogen }\end{array}$ \\
\hline 50 & Clec $1 \mathrm{~b}$ & C-type lectin domain family 1, member b [CLEC-2] & MGI:1913287 & BHP & -1 & 7 & 0 & 4 & $\begin{array}{l}\downarrow \text { PLT activ. with podoplanin; } \\
\text { abnormal lymph EC }\end{array}$ \\
\hline 51 & Cpb2 & $\begin{array}{l}\text { carooxypeptidase } \mathrm{B} 2 \text { (thrombin-activatable fibrinolysis inhibitor, } \\
\text { TAFl) }\end{array}$ & MGI:1891837 & A & 0 & 4 & 0 & 5 & unchanged fibrinolysis \\
\hline 52 & Ctsg & cathepsin G & MG1:88563 & A & -1 & 2 & -1 & 2 & $\begin{array}{l}\downarrow \text { formation of neutrophil-PLT } \\
\text { aggregates }\end{array}$ \\
\hline 53 & Cxcr6 & chemokine C-X-C motif receptor 6 [CXCR6] & MGI:1934582 & A & 1 & 1 & & & $\begin{array}{l}\downarrow \text { PLT aggr, integrin activ. and } \\
\text { secr. with CXCL-16 }\end{array}$ \\
\hline 54 & Cybb & cytochrome b-245, beta polypeptide (Nox2) & MG1:88574 & A & -1 & 1 & & & $\downarrow$ PLT activ. with collagen \\
\hline 55 & Dct & dopachrome tautomerase (slaty) & MGI:102563 & $\mathrm{A} / \mathrm{m}$ & & & 0 & 1 & mouse: abnormal pigmentation \\
\hline 56 & Dock7 & dedicator of cytokines is 7 (misty) & MGI:1914549 & $\mathrm{A} / \mathrm{m}$ & & & -1 & 1 & $\begin{array}{l}\text { mouse: abnormal pigmentation; } \\
\downarrow \text { PLT ADP storage }\end{array}$ \\
\hline 57 & Dtnbp1 & dystrobrevin binding protein 1 (sandy) & MGI:2137586 & $\mathrm{A} / \mathrm{m}$ & & & -1 & 1 & $\begin{array}{l}\downarrow \text { PLT aggr. and secr. with } \\
\text { collagen, thrombin }\end{array}$ \\
\hline 58 & Edaradd & $\begin{array}{l}\text { EDAR ectodyspla sin-A receptor-associated death doma in } \\
\text { (crinkled) }\end{array}$ & MGI:1931001 & $\mathrm{A} / \mathrm{m}$ & & & 0 & 1 & mouse: $\downarrow$ viability \\
\hline 59 & Elane (Ctsg) & ela stase + cathepsin G & MGI:2679229 & A & -1 & 1 & -1 & 1 & double $\mathrm{KO}: \downarrow$ proteolysis of TFPI \\
\hline 60 & Entpd1 & ectonucleoside triphosphate diphosphohydrolase 1 (CD39) & MGI:102805 & A & -2 & 1 & -1 & 1 & $\begin{array}{l}\downarrow \text { degradation by EC of ADP and } \\
\text { ATP }\end{array}$ \\
\hline 61 & Esam & endothelial cell-specific achesion molecule (ESAM) & MGI:1916774 & A & 2 & 1 & 1 & 1 & $\uparrow P L T$ aggr., $\downarrow$ disaggr. \\
\hline 62 & F10 & coagulation factor $X$ & MGI:103107 & B & -2 & 2 & -1 & 2 & $\downarrow$ coagulation \\
\hline 63 & F11 & coagulation factor $X I$ & MG1:99481 & A & -1 & 8 & 0 & 4 & $\downarrow$ coagulation \\
\hline 64 & F11r & F11 receptor (IAM-A) & MGI:1321398 & A & 2 & 3 & 1 & 1 & $\uparrow$ PLT activ. (integrin signa ling) \\
\hline 65 & F12 & coagulation factor XII & MGI:1891012 & ABL & -2 & 10 & 0 & 3 & $\downarrow$ coagulation (intrinsic pathway) \\
\hline 66 & F13a1 & coagulation factor XIII, A1 subunit & MGI:1921395 & A & & & -1 & 1 & $\downarrow$ clot stability \\
\hline 67 & F2 & coagulation factor II (prothrombin) & MG1:88380 & B & -2 & 6 & -1 & 1 & $\begin{array}{l}\downarrow \text { coagulation and fibrin } \\
\text { formation }\end{array}$ \\
\hline 68 & F2r & $\begin{array}{l}\text { coagulation factor II thrombin receptor (protease-activated } \\
\text { receptor 1, PAR1) }\end{array}$ & MGI:101802 & A & 0 & 1 & 0 & 1 & $\begin{array}{l}\text { no clear PLT phenotype; } \downarrow \text { EC } \\
\text { activ. with thrombin }\end{array}$ \\
\hline 69 & F2r12 & $\begin{array}{l}\text { coagulation factor Il thrombin receptor-like } 2 \text { (protease-activated } \\
\text { receptor } 3 \text {, PAR3) }\end{array}$ & MGI:1298208 & A & -1 & 3 & -1 & 2 & $\downarrow$ PLT activ. with thrombin \\
\hline 70 & F2ri3 & $\begin{array}{l}\text { coagulation factor II thrombin receptor-like } 3 \text { (protease-activated } \\
\text { receptor } 4 \text {, PAR4) }\end{array}$ & MGI:1298207 & A & -1 & 9 & -1 & 3 & $\downarrow$ PLT activ. with thrombin \\
\hline
\end{tabular}




\begin{tabular}{|c|c|c|c|c|c|c|c|c|c|}
\hline 71 & F3 & coagulation factor III (tissue factor) & MGI:88381 & ABS & -2 & 5 & & & $\downarrow$ extrinsic coagulation \\
\hline 72 & F5 & coagulation factor $\mathrm{V}$ & MGI:88382 & BLP & -1 & 4 & -1 & 2 & $\begin{array}{l}\text { spont. bleeding pre- and peri- } \\
\text { natal }\end{array}$ \\
\hline 73 & F7 & coagulation factor VII & MGI:109325 & 8 & -2 & 1 & & & $\begin{array}{l}\downarrow \text { coagulation (extrinsic } \\
\text { pathway) }\end{array}$ \\
\hline 74 & F8 & coagulation factor VIII & MGI:88383 & A & -2 & 5 & -1 & 18 & $\downarrow$ coagulation \\
\hline 75 & F9 & coagulation factor IX & MGI:88384 & A & -2 & 5 & -1 & 7 & $\downarrow$ coagulation \\
\hline 76 & Fbn1 & fibrillin 1 (tight skin) & MG1:95489 & $\mathrm{A} / \mathrm{m}$ & & & 0 & 1 & mouse: abnormal skin \\
\hline 77 & Fcerlg & $\begin{array}{l}\text { Fc receptor, IgE, high affinity I, gamma polypeptide (FcR gamma } \\
\text { chain) }\end{array}$ & MGI:95496 & A & -2 & 16 & -1 & 4 & $\begin{array}{l}\downarrow \text { PLT adh., activ. with collagen, } \\
\text { VWF }\end{array}$ \\
\hline 78 & Fcgrab & Fc receptor, IgG, low affinity IIb (Fcgr2a) & MG1:95500 & A & -1 & 1 & 0 & 1 & $\uparrow$ PLT activ. on fibrinogen, $\uparrow C R$ \\
\hline 79 & Fermt2 & fermitin family homolog 2 (Kndin-2) & MGI:2385001 & A & & & -1 & 1 & $\begin{array}{l}\uparrow \text { enco-nucleotidase activity on } \\
\text { EC }\end{array}$ \\
\hline 80 & Fermt3 & fermitin family homolog 3 (Kndlin-3) & MGI:2147790 & H & -2 & 1 & -1 & 1 & $\downarrow$ PLT aggr, integrin activ. \\
\hline 81 & $\mathrm{Fgg}$ & fibrinogen gamma chain & MG1:95526 & A & -1 & 4 & -1 & 2 & $\begin{array}{l}\downarrow \text { PLT aggr:; } \uparrow \text { plasma } \\
\text { fibronectin }\end{array}$ \\
\hline 82 & $\mathrm{Fgr}$ & Gardner-Rasheed feline sarcoma viral $\mathrm{Fgr}$ oncogene homolog ( $\mathrm{Fgr}$ ) & MG1:95527 & A & 0 & 1 & 0 & 1 & no PLT phenotype \\
\hline 83 & Fll1 & Friend leukemia integration 1 (SIC-1) & MG1:95554 & A & & & -1 & 1 & $\begin{array}{l}\text { thrombocytopenia; } \downarrow \text { PLT activ., } \\
\text { aggr. }\end{array}$ \\
\hline 84 & FIna & filamin, alpha (fila min $A$ ) & MGl:95556 & $\mathrm{H}$ & -2 & 1 & -1 & 1 & $\begin{array}{l}\text { macrothrombocytopenia; } \downarrow \text { PLT } \\
\text { activ. with collagen, CLEC2 }\end{array}$ \\
\hline 85 & Fn1 & fibronectin 1 & MGI:95566 & A & -1 & 2 & 0 & 2 & $\downarrow P L T$ aggr. \\
\hline 86 & Fyb & FYN binding protein (ADAP) & MGI:1346327 & A & -1 & 3 & 0 & 1 & $\downarrow$ PLT spreading under flow \\
\hline 87 & Fyn & Fyn proto-oncogene & MG1:95602 & A & 0 & 1 & 0 & 2 & no PLT phenotype \\
\hline 88 & Gas6 & growth-arrest spefic 6 [Gas6) & MG1:95660 & A & -1 & 3 & 0 & 1 & $\downarrow$ PLT aggr. with weak agonists \\
\hline 89 & Gata1 & GATA binding protein 1 & MGI: 95661 & $p$ & -2 & 1 & & & $\begin{array}{l}\text { thrombocytoperia; } \downarrow \text { PLT activ. } \\
\text { with collagen }\end{array}$ \\
\hline 90 & Gdf15 & growth differentiation factor 15 & MGI:1346047 & A & 2 & 2 & 1 & 1 & $\uparrow$ PLT integrin activ. \\
\hline 91 & Gla4 & gap junction protein, alpha 4 (connexin 37) & MG1:95715 & A & 1 & 3 & 1 & 2 & $\begin{array}{l}\downarrow P L T \text { aggr:; } \downarrow \text { secr. and gap } \\
\text { junction formation }\end{array}$ \\
\hline 92 & G]a5 & gap junction protein, alpha 5 (connexin 40) & MGI:133807 & A & & & -1 & 1 & $\downarrow$ PLT secr., aggr. with collagen \\
\hline 93 & Gna12 & guanine nucleotide binding protein, alpha 12 (G12 alpha) & MG1:95767 & A & -1 & 2 & 0 & 1 & $\begin{array}{l}\text { no clear PLT phenotype, } \downarrow \text { EC } \\
\text { VWF secr. }\end{array}$ \\
\hline 94 & Gna13 & guanine nucleotide binding protein, alpha 13 (G13 alpha) & MGl:95768 & A & -2 & 3 & -1 & 1 & $\begin{array}{l}\downarrow P L T \text { activ. with thrombin, TXA, } \\
\text { collagen }(\downarrow \text { act } v \text {. of RhoA) }\end{array}$ \\
\hline 95 & Gnal2 & guanine nucleotide binding protein, alpha inhibiting 2 ( $\mathrm{G}$ i2 alpha) & MGI:95772 & $\mathrm{AH}$ & 1 & 3 & & & $\begin{array}{l}\uparrow P L T \text { activ. with } A D P \text {, thrombin, } \\
\text { TXA, collagen }\end{array}$ \\
\hline 96 & Gnaq & guanine nucleotide binding protein, alpha q polypeptide (Gq alpha) & MGI:95776 & AB & -2 & 5 & -1 & 2 & $\begin{array}{l}\downarrow P L T \text { activ. with thrombin, TXA, } \\
\text { ADP }\end{array}$ \\
\hline 97 & Gnaz & guanine nucleotide binding protein, alpha 2 subunit ( $\mathrm{G} z$ alpha) & MGI:95780 & A & -1 & 3 & -1 & 1 & $\downarrow$ PLT activ. with epinephrine \\
\hline 98 & Gp1ba & glycoprotein 1b, alpha polypeptide (GPIb alpha, CD42b) & MGI:1333744 & AB & -2 & 5 & -1 & 1 & $\begin{array}{l}\text { macrothrombocytopenia; } \downarrow \text { PLT } \\
\text { adh., activ. under flow }\end{array}$ \\
\hline 99 & Gp1bb & glycoprotein $1 \mathrm{~b}$, beta polypeptide (GPIb beta, CD42c) & MGI:107852 & A & -1 & 6 & -1 & 2 & large PLTs; $\downarrow$ WWF binding to PLTs \\
\hline 100 & Gp5 & glycoprotein 5 platelet (GPV, CD42d) & MGI:1096363 & A & 0 & 6 & 0 & 2 & $\begin{array}{l}\downarrow \text { PLT adh. and activ. with } \\
\text { collagen; } \uparrow \text { act v. with thrombin }\end{array}$ \\
\hline 101 & Gp6 & glycoprotein 6 platelet (GPVI) & MGI:1889810 & $\mathrm{AB}$ & -2 & 24 & 0 & 5 & $\downarrow$ collagen-induced PLT activ. \\
\hline 102 & Gpr161 & G protein-coupled receptor 161 (vacuolated lens) & MGI:2685054 & $\mathrm{A} / \mathrm{m}$ & & & 0 & 1 & mouse: embryonal lethality \\
\hline 103 & Gpx1 & glutathione peroxidase 1 & MGI:104887 & A & 1 & 1 & & & $\uparrow P L T$ integrin function \\
\hline 104 & Gpx3 & glutathione peroxidase 3 & MGI:105102 & A & 2 & 1 & 1 & 1 & $\uparrow$ PLT activ. with ADP \\
\hline 105 & Gpx4 & glutathione peroxidase 4 & MGI:10476 & $\mathrm{E}$ & 1 & 1 & & & $\begin{array}{l}\text { multiorgan thrombus formation } \\
\text { (vit. E depletion) }\end{array}$ \\
\hline 106 & Grb2 & growth factor receptor bound protein 2 [Grb2] & MGI:95805 & $p$ & -1 & 5 & -1 & 2 & $\begin{array}{l}\downarrow \text { PLT activ. with collagen, } \\
\text { rhodocytin }\end{array}$ \\
\hline 107 & Grial & glutamate receptor, ionotropic, AMPA1 alpha 1 (AMPAA) & MG1:95808 & AB & -2 & 2 & -1 & 1 & $\begin{array}{l}\downarrow N a \text { influx in PLTs with } \\
\text { glutamate }\end{array}$ \\
\hline 108 & Grik2 & glutamate receptor, ionotropic, ka inate 2 beta 2 & MGI:95815 & A & -1 & 1 & & & $\downarrow$ PLT responses with glutamate \\
\hline 109 & Gsk3b & glycogen synthase kinase 3 beta (GSK) & MGI:1861437 & A & 2 & 2 & & & $\uparrow$ PLT activ. with thrombin \\
\hline 110 & Gucy1b3 & guanylate cyclase 1 , soluble, beta 3 & MGI:1860604 & $p$ & -1 & 1 & -1 & 2 & $\begin{array}{l}\downarrow \text { PLT activ. with NO, thrombin, } \\
\text { collagen }\end{array}$ \\
\hline
\end{tabular}




\begin{tabular}{|c|c|c|c|c|c|c|c|c|c|}
\hline 111 & Hels1 & hematopoietic specific Lyn substrate 1 (HS1) & MGl:104568 & A & -1 & 2 & 0 & 2 & unchanged PLT activ. \\
\hline 112 & Hmox1 & heme oxygenase decycling 1 & MGI:96163 & $\mathrm{AH}$ & 1 & 4 & 0 & 1 & $\begin{array}{l}\text { no degradation of heme; } \\
\text { damaged EC; } \uparrow \text { plasma VWF }\end{array}$ \\
\hline 113 & Hmtb11 (QTL) & quantitative trait locus $\triangle \mathrm{A}-2$ & - & A & -2 & 1 & 0 & 1 & $\begin{array}{l}\text { quantitat ve trait locus for } \downarrow \\
\text { arterial thrombosis, bleeding }\end{array}$ \\
\hline 114 & Hpn & hepsin & MGI:1196620 & A & & & 0 & 1 & unchanged coagulation \\
\hline 115 & Hps1 & Hermansky-Pudlak syndrome 1 homolog (pale ear) & MGI:2177763 & $\mathrm{A} / \mathrm{m}$ & & & -1 & 2 & $\downarrow$ granule content and ATP secr. \\
\hline 116 & Hps3 & Hermansky-Pudlak syndrome protein 3 & MGI:2153839 & A & -1 & 1 & -1 & 2 & $\begin{array}{l}\downarrow \text { PLT secr. (dense granules); } \downarrow \\
\text { PLT-WBC interaction }\end{array}$ \\
\hline 117 & Hps4 & Hermansky-Pudlak syndrome 4 homolog (light-ear) & MGI:2177742 & $\mathrm{A} / \mathrm{m}$ & & & -1 & 1 & $\downarrow_{\text {granule content and ATP secr. }}$ \\
\hline 118 & Hps5 & Hermansky-Pudlak syndrome 5 homolog (maroon) & MGI:2180307 & $\mathrm{A} / \mathrm{m}$ & & & -1 & 1 & $\begin{array}{l}\text { PLT dense granule |lysosomal\} } \\
\text { defect; } \uparrow \text { clotting time }\end{array}$ \\
\hline 119 & Hps6 & Hermansky-Pudlak syndrome 6 (ruby-eye) & MGI:2181763 & A & -1 & 1 & -1 & 1 & $\begin{array}{l}\text { no PLT dense granules, } \downarrow \text { PLT } \\
\text { secr. }\end{array}$ \\
\hline 120 & Hrg & histidine-rich glycoprotein & MGI:2146636 & A & & & 1 & 1 & $\uparrow$ coagulation and fibrinolysis \\
\hline 121 & Hsp90b1 & heat shock protein 90, beta, member 1 (GRP94, go96, ERp99) & MGI:98817 & A & & & -1 & 1 & macrothrombocytopenia \\
\hline 122 & Icam1 & intercellular adhesion molecule 1 (Icam1, CD54) & MGI:96392 & A & -1 & 1 & & & $\downarrow$ WBC-EC adh. \\
\hline 123 & Ikbkb & inhibitor of kappa B kinase beta (IKK-beta) & MGI:133807 & P & & & -1 & & altered PLT phosphorylation \\
\hline 124 & llk & integrin linked kinase & MGI:1195267 & A & -1 & 1 & -1 & 1 & $\begin{array}{l}\downarrow \text { PLT secr., aggr. with collagen } \\
\text { thrombin }\end{array}$ \\
\hline 125 & Inpp5d & inositol polyphosphate-5-phosphatase D (SHIP1) & MGI:107357 & A & -2 & 2 & -1 & 1 & $\begin{array}{l}\downarrow \text { PLT aggr. with thrombin } \\
\text { collagen; } \downarrow \text { CR }\end{array}$ \\
\hline 126 & Ip6k1 & inositol hexasphosphate kinase 1 & MGI:1351633 & A & -1 & 1 & -1 & 1 & $\downarrow$ PLT aggr., $\uparrow$ clotting time \\
\hline 127 & Itga2 & integrin alpha 2 (CD49b) & MGI:96600 & $A B$ & -1 & 6 & 0 & 4 & $\downarrow$ PLT adh. to collagen \\
\hline 128 & Itga2b & integrin alpha $2 \mathrm{~b}$ (alphallb, GPIIb, CD 41 ) & MGl:96601 & A & & & -1 & 1 & $\downarrow$ MGK maturation; $\downarrow$ PLT aggr. \\
\hline 129 & Itga6 & integrin alpha 6 & MGI:96605 & P & -2 & 3 & 0 & 1 & $\downarrow$ PLT adh. to laminin \\
\hline 130 & Itgb1 & integrin beta 1 (CD29) & MGI:96610 & H & -1 & 6 & 0 & 2 & $\begin{array}{l}\downarrow \text { PLT adh. to collagen and } \\
\text { laminin }\end{array}$ \\
\hline 131 & Itgb3 & integrin beta3 [GPIIIa, CD61] & MGI:96612 & $A B$ & -2 & 10 & -1 & 8 & general defect in PLT aggr. \\
\hline 132 & Jak2 & Janus kinase 2 (Jak2) & MGI:96629 & H & & & 1 & 1 & unchanged PLT activ. count \\
\hline 133 & Kсnj6 & $\begin{array}{l}\text { potassium irwardly-rectifying channel, subfamily J, member } 6 \\
\text { (weaver) }\end{array}$ & MGI:104781 & $\mathrm{A} / \mathrm{m}$ & & & 0 & 1 & mouse: $\downarrow$ weight \\
\hline 134 & Kit & kit oncogene (viable dominant spotting) & MGI:96677 & $\mathrm{A} / \mathrm{m}$ & & & 0 & 1 & mouse: abnormal hematopoiesis \\
\hline 135 & Klk4 & kallikrein related-peptidase 4 (kallikrein) & MGI:1861379 & $\mathrm{AL}$ & -2 & 2 & -1 & 1 & $\begin{array}{l}\uparrow \text { plasma factor XII, } \uparrow \text { clotting } \\
\text { time }\end{array}$ \\
\hline 136 & Kng1 & kininogen 1 & MGI:1097705 & A & -1 & 1 & 0 & 1 & $\begin{array}{l}\text { no low-molecular-weight } \\
\text { kininogen; } \downarrow \text { coagulation }\end{array}$ \\
\hline 137 & Lat & linker for activation of $T$ cells (LAT) & MGI:1342293 & A & -2 & 3 & & & $\begin{array}{l}\downarrow \text { act } v \text {. with collagen, other } \\
\text { agonists }\end{array}$ \\
\hline 138 & Lcp2 & Iymphocyte cytosolic protein 2 (SLP-76) & MGI:1321402 & A & -2 & 6 & & & $\downarrow$ PLT activ. with collagen \\
\hline 139 & Ldilr & low density lipoprotein receptor (LDL receptor) & MGI:96765 & A & 1 & 2 & & & $\uparrow$ plasma cholesterol \\
\hline 140 & Lep & leptin & MGl:104663 & $A B$ & -1 & 2 & & & 个PA -1 , TF in plasma \\
\hline 141 & Lepr & leptin receptor & MGI:104993 & B & -1 & 1 & 0 & 1 & $\begin{array}{l}\text { high plasma leptin; unchanged } \\
\text { PLT activ. }\end{array}$ \\
\hline 142 & Lgals1 & lectin, galactose binding, soluble 1 (galectin-1) & $M G I: 96777$ & A & & & -1 & 1 & $\begin{array}{l}\downarrow \text { PLT spread., secr.; unchanged } \\
\text { PLT aggr. }\end{array}$ \\
\hline 143 & Limk1 & LIM-domain containing, protein kinase (LIM kinase 1) & MGI:104572 & A & -1 & 1 & 0 & 1 & $\downarrow$ PLT activ. with GPIb-VWF \\
\hline 144 & Lipa & Iysosomal acid lipase A & MGI:96789 & A & -2 & 1 & -1 & 1 & likely $\downarrow$ PLT activ. \\
\hline 145 & Lnpep & $\begin{array}{l}\text { leucy//cystinyl aminopeptidase (insulin-regulated aminopeptidase, } \\
\text { IRAP) }\end{array}$ & MGI:2387123 & A & -1 & 1 & & & $\uparrow$ fibrinolysis with angotensins \\
\hline 146 & Lrp8 & low density lipoprotein receptor-related protein 8 , apoli poprote in & MGI:1340044 & A & -1 & 2 & 0 & 1 & likely $\downarrow$ PLT activ. \\
\hline 147 & Lyn & Yamaguch sarcoma viral oncogene homolog (LYn) & MGI:96892 & A & -2 & 2 & 0 & 1 & $\uparrow$ PLT spreading and aggr. \\
\hline 148 & Lyst & Iysosomal trafficking regulator (beige) & MGI:107448 & $\mathrm{A} / \mathrm{m}$ & & & -1 & 4 & lysosomal dysfunction \\
\hline 149 & Mapk14 & mitogen-activated protein kinase 14 (p38 alpha MAPK) & MGI:1346865 & A & -1 & 1 & 0 & 1 & $\begin{array}{l}\downarrow \text { PLT activ. by TXA; } \downarrow \text { TF in } \\
\text { vessel wall }\end{array}$ \\
\hline 150 & Mapk8 & mitogen-activated protein kinase 8 (Mapk8, JNK1) & MGI:1346861 & AB & -2 & 4 & -1 & 1 & $\begin{array}{l}\downarrow \text { PLT (integrin) actv. with } \\
\text { various agonists }\end{array}$ \\
\hline
\end{tabular}




\begin{tabular}{|c|c|c|c|c|c|c|c|c|c|}
\hline 151 & Mas1 & MAS1 oncogene & MG1:96918 & A & & & 1 & 1 & $\downarrow$ angiotensin binding to PLTs \\
\hline 152 & Masp1 & $\begin{array}{l}\text { mannan-binding lectin serine peptidase } 1 \text { (MBL-associated serine } \\
\text { protease) }\end{array}$ & MG1:88492 & A & -2 & 1 & & & $\downarrow$ complement activ:; $\downarrow$ TS \\
\hline 153 & Mbl1 (Mblz) & mannose-binding lectin protein $A+C$ & MG1:96923 & A & -2 & 1 & & & $\begin{array}{l}\downarrow \text { complement activ., } \downarrow \text { PLT } \\
\text { aggr., } \downarrow \text { TS }\end{array}$ \\
\hline 154 & Mbl2 (Mbl1) & mannose-binding lectin protein $\mathrm{C}+\mathrm{A}$ & MG1:96924 & A & -2 & 1 & & & $\begin{array}{l}\downarrow \text { complement activ., } \downarrow \text { PLT } \\
\text { aggr., } \downarrow \text { TG }\end{array}$ \\
\hline 155 & Mclif & melanocortin 1 receptor (sombre) & MGI:99456 & $\mathrm{A} / \mathrm{m}$ & & & 0 & 1 & mouse: abnormal pigmentation \\
\hline 156 & Mertk & c-mer proto-oncogene tyrosine kinase (Mer) & MG1:96965 & A & -1 & 4 & -1 & 3 & $\downarrow$ PLT aggr. with weak agonists \\
\hline 157 & Mfap2 & microfibrillar-associated protein 2 (MAGP1) & MGI:99559 & $\bar{A}$ & -1 & 2 & -1 & 1 & $\begin{array}{l}\text { altered ECM; } \downarrow \text { PLT count; } \\
\text { unchanged PLT activ. }\end{array}$ \\
\hline 158 & Mgrn1 & mahogunin, nng finger 1 (mahoganoid) & MGI:2447670 & $\mathrm{A} / \mathrm{m}$ & & & 0 & 1 & mouse: abnormal pigmentation \\
\hline 159 & Mitf & microphtha Imia-associated transcription factor (white) & MGI:104554 & $\mathrm{A} / \mathrm{m}$ & & & 1 & 1 & mouse: hypopigmentation \\
\hline 160 & Mkl1 (Mkl2) & megakaryobla stic leukemia, myocardir-like 1 (Mk|1) & MGI:2384495 & $\overline{A P}$ & & & 0 & 2 & $\begin{array}{l}\downarrow \text { PLT act } v \text {. with ADP; altered } \\
\text { grotein expression }\end{array}$ \\
\hline 161 & Mlk2 & megakaryoblastic leukemia, myocardin-like 2 (Mkl2) & MGI:3050795 & $\mathbf{p}$ & & & 0 & 1 & no PLT phenotype \\
\hline 162 & Mlph & melanophilin (leaden, fuzzy) & MGI:2176380 & $\mathrm{A} / \mathrm{m}$ & & & 1 & 1 & mouse: abnormal hematopoiesis \\
\hline 163 & Mmp10 & matrix metallopeptidase 10 (stromelysin-2) & MG1:97007 & A & 2 & 1 & 1 & 1 & $\downarrow$ filonnolysis \\
\hline 164 & Mmp2 & matrix metallopeptidase 2 & MGI:97009 & A & -1 & 3 & -1 & 1 & $\begin{array}{l}\downarrow \text { PLT actv. with collagen, } \\
\text { thrombin, ADP }\end{array}$ \\
\hline 165 & Mmp3 & matrix metallopeptidase 3 & MG1:97010 & A & 0 & 1 & & & no clear phenotype in PLTs \\
\hline 166 & Mmp9 & matrix metallopeptidase 9 & MG1:97011 & A & 1 & 1 & & & $\uparrow$ PLT actv. with collagen \\
\hline 167 & Mrvil & MRV integration site 1 (IRAG) & MGI:1338023 & A & 0 & 1 & 0 & 2 & $\begin{array}{l}\downarrow \text { PLT NO inhibition; lower BP; } \downarrow \\
\text { heart contraction }\end{array}$ \\
\hline 168 & Myh9 & myosin, heavy polypeptide 9, non-muscle (myosin heavy cha in 9) & $M G 1: 107717$ & $\mathbf{p}$ & -1 & 2 & -1 & 1 & $\begin{array}{l}\text { thrombocytopenia; } \downarrow \text { fibrinogen } \\
\text { activ.; } \downarrow \text { CR }\end{array}$ \\
\hline 169 & Myo5a & myosin VA (dilute) & MGl:105976 & $\mathrm{N} / \mathrm{m}$ & & & 0 & 1 & mouse: abnormal pigmentation \\
\hline 170 & Nbeal2 & neurobeachin-like 2 (Nbea|2) & MGI:2448554 & $\bar{A}$ & -2 & 4 & -1 & 2 & $\begin{array}{l}\text { no PLT alpha-granules, } \downarrow \text { PLT } \\
\text { adh., aggr., coagulant activity }\end{array}$ \\
\hline 171 & Nfez & nuclear factor, erythroid-denved 2 (NFED-2) & MGI:97308 & A & -1 & 1 & -1 & 1 & thrombocytopenia \\
\hline 172 & Nos2 & nitric oxide synthase 2 , inducible (iNOS) & MGI:97361 & A & 1 & 2 & -1 & 1 & $\begin{array}{l}\uparrow \text { urinary nitrite (female): } \\
\text { unchanged PLT activ. }\end{array}$ \\
\hline 173 & Nos3 & nitric oxide synthase 3, endothelial cell (eNOS) & MGI:97362 & A & 0 & 3 & 1 & 2 & $\begin{array}{l}\uparrow \text { plasma } t P A \text { and WWF: } \uparrow \\
\text { fibrinolysis }\end{array}$ \\
\hline 174 & Nr1h2-ps & $\begin{array}{l}\text { nuclear receptor subfamily } 1 \text {, group } H \text {, member } 2 \text {, pseudogene } \\
\text { (liver } X \text { receptor beta, LXR-beta) }\end{array}$ & MGI:1354955 & B & -2 & 1 & -1 & 1 & $\downarrow$ PLT act v. with collagen \\
\hline 175 & Nt5e & $S^{\prime}$ nuc leotidase, ecto $[\mathrm{CD} 73)$ & MG1:99782 & A & 1 & 1 & 0 & 1 & $\begin{array}{l}\text { lower PLT CAMP level; unchanged } \\
\text { activ. PLTs }\end{array}$ \\
\hline 176 & Ocaz & oculocutaneous albinism II (pink-eyed) & MG1:97454 & $\mathrm{A} / \mathrm{m}$ & & & 0 & 1 & mouse: abnormal pigmentation \\
\hline 177 & Orai1 & ORAl calcium release-activated calcium modulator 1 & MGI:1925542 & $\mathrm{H}$ & -1 & 7 & -1 & 1 & $\begin{array}{l}\downarrow \text { Ca entry and PLT activ. with } \\
\text { multiple agonists }\end{array}$ \\
\hline 178 & Ostm1 & osteopetrosis associated transmembrane prote in 1 (gray) & MGI:2655574 & $\mathrm{A} / \mathrm{m}$ & & & 0 & 1 & mouse: abnormal pigmentation \\
\hline 179 & P2rx1 & $\begin{array}{l}\text { purinergic receptor P2X, ligand-gated ion channel, } 1 \text { (P2X1 } \\
\text { receptor) }\end{array}$ & MGI:1098235 & A & -2 & 5 & 0 & 1 & $\downarrow$ PLT actv. with ATP \\
\hline 180 & P2rx7 & $\begin{array}{l}\text { purinergic receptor P2X, ligand-gated ion channel, } 7 \text { (P2X7 } \\
\text { receptor) }\end{array}$ & MGI:1339957 & $\mathrm{AH}$ & -1 & 4 & & & $\begin{array}{l}\downarrow \text { procoagulant activity of } \\
\text { myeloid cells }\end{array}$ \\
\hline 181 & P2ry1 & purinergic receptor P2Y, G-protein coupled 1 (P2Y1 receptor) & $M G 1: 105049$ & A & -1 & 7 & -1 & 3 & $\downarrow$ PLT act v. with ADP \\
\hline 182 & P2yr12 & purinergic receptor P2Y, G-protein coupled 12 (P2Y12 receptor) & MGI:19180Bg & $A B L$ & -2 & 10 & -1 & 3 & $\downarrow$ PLT actv. with ADP \\
\hline 183 & Pde3a & phosphodiesterase 3A, CGMP inhibited & MGI:1860764 & A & -1 & 1 & & & elevated CAMP in PLTs \\
\hline 184 & Pde3b & phosphodiesterase 3B, cGMP-inhibited & MGI:1333863 & $\bar{A}$ & 0 & 1 & & & $\begin{array}{l}\text { no clear PLT phenotype (normal } \\
\text { CAMP) }\end{array}$ \\
\hline 185 & Pdia3 & protein disulfide isomera se associated 3 (ERp57) & MGI:95834 & BP & -2 & 4 & -1 & 2 & $\downarrow$ PLT integrin actvv. and aggr. \\
\hline 186 & Pdilim1 & PDZ and LIM domain 1 (CLP-36) & MGI:1860611 & $\bar{A}$ & 1 & 3 & 0 & 1 & 个 PLT GPVI signaling \\
\hline 187 & Pdllim7 & PDZ and LIM domain 7 & MGI:1914649 & A & 1 & 1 & 1 & 1 & $\begin{array}{l}\text { cardiac dysfunction; spont. } \\
\text { arterial/venous thrombosis }\end{array}$ \\
\hline 188 & Pdpk1 & 3-phosphoinositide dependent protein kinase 1 [POK1) & MGI:1338058 & $\mathbf{p}$ & -1 & 1 & & & $\begin{array}{l}\downarrow \text { PLT agrgegation with thrombin, } \\
\text { ADP, TXA }\end{array}$ \\
\hline 189 & Pecam1 & platelet/endothelial cell adhesion molecule 1 (Pecam1, CD31) & MG1:97537 & $\widehat{\mathrm{AH}}$ & 1 & 5 & 0 & 1 & $\uparrow$ PLT act $v$. with collagen \\
\hline 190 & Per2 & period circadian clock 2 (Per2) & MGI:1195265 & A & & & -1 & 1 & $\begin{array}{l}\text { thrombocytopenia; } \downarrow \text { PLT aggr. } \\
\text { and secr. }\end{array}$ \\
\hline
\end{tabular}




\begin{tabular}{|c|c|c|c|c|c|c|c|c|c|}
\hline 191 & $\mathrm{Pf} 4 \mathrm{~A}$ & platelet factor 4 & MGI:1888711 & A & -2 & 1 & 0 & 1 & $\begin{array}{l}\text { no PLT phenotype; anticoagulant } \\
\text { effect unclear (heparin) }\end{array}$ \\
\hline 192 & Plk3ca & $\begin{array}{l}\text { phosphatidylinositol 3-kinase, catalytic, alpha polypeptide [PI3K } \\
\text { alpha) }\end{array}$ & MGI:1206581 & $\mathrm{p}$ & -1 & 2 & & & $\downarrow$ PLT activ. with collagen \\
\hline 193 & Plk3cb & $\begin{array}{l}\text { phosphatidylinositol 3-kinase, catalytic, beta polypeptide [P] } 3 \mathrm{~K} \\
\text { beta) }\end{array}$ & MGI:1922019 & $A B P$ & -2 & 7 & -1 & 2 & $\begin{array}{l}\downarrow \text { PLT activ. with ADP, collagen; } \\
\downarrow \text { CR }\end{array}$ \\
\hline 194 & Plk3cg & $\begin{array}{l}\text { phosphatidylinositol 3-kinase, catalytic, beta polypeptide [PI3K } \\
\text { gamma) }\end{array}$ & MGI:1353576 & A & -1 & 4 & 0 & 2 & $\downarrow$ PLT integrin act v. with ADP \\
\hline 195 & Plk3r1 & $\begin{array}{l}\text { phosphatidylinositol 3-kinase, regulatory subunit, polypeptide } 1 \\
\text { (p85 alpha) }\end{array}$ & MG1:97583 & A & -2 & 1 & & & $\downarrow$ PLT activ. with collagen \\
\hline 196 & Plp5k1b & $\begin{array}{l}\text { phosphatidylinositol-4-phosphate 5-kinase, type } 1 \text { beta [PIP5KI } \\
\text { beta) }\end{array}$ & MGI:107930 & A & -1 & 1 & & & $\begin{array}{l}\downarrow \text { PLT activ. with thrombin, TxA; } \\
\uparrow \text { disaggr. }\end{array}$ \\
\hline 197 & Plp5k1c & $\begin{array}{l}\text { phosphatidylinositol-4-phosphate 5-kinase, type } 1 \text { gamma (PIPSK1 } \\
\text { gamma) }\end{array}$ & MGI:1298224 & P & -1 & 1 & & & $\downarrow$ PLT integrin function \\
\hline 198 & Pla2g4a & $\begin{array}{l}\text { phospholipase A2, group IVA, cytosolic, calcium-dependent } \\
\text { (CPLA2) }\end{array}$ & MGI:1195256 & A & -1 & 1 & -1 & 1 & $\downarrow$ PLT activ. with collagen \\
\hline 199 & Plat & $\begin{array}{l}\text { plasminogen activator, tissue [tissue-type plasminogen activator, } \\
\text { tPA] }\end{array}$ & MGl:97610 & A & 0 & 3 & 0 & 3 & $\downarrow$ fibrinolysis \\
\hline 200 & Plaur & plasminogen activator, urokinase receptor (urokinase) & MGI:97612 & A & 0 & 3 & 0 & 3 & $\downarrow$ fibrinolysis \\
\hline 201 & Plcb2 (Plcb3) & phospholipase C, beta $2+$ beta 3 (PLC beta $2+3$ ) & MGI:107465 & A & -2 & 1 & & & $\begin{array}{l}\downarrow \text { PLT activ. with thrombin, ADP, } \\
\text { TXA }\end{array}$ \\
\hline 202 & Plcb3 (Plcb2) & phospholipase $\mathrm{C}$, beta $3+$ beta 2 (PLC beta $2+3$ ) & MGI:96600 & A & -2 & 1 & & & $\begin{array}{l}\downarrow \text { PLT activ. with thrombin, ADP, } \\
\text { TXA }\end{array}$ \\
\hline 203 & Plcg2 & phospholipase C, gamma 2 (PLC gamma 2) & MG1:97616 & A & -2 & 4 & -1 & 1 & $\begin{array}{l}\downarrow \text { PLT activ. with collagen, } \\
\text { fibrinogen, VWF, CLEC-2 }\end{array}$ \\
\hline 204 & Pld1 & phospholipase D1 & MGI:109585 & A & -1 & 6 & 0 & 2 & $\begin{array}{l}\downarrow \text { PLT integrin act } v \text {., } \downarrow \text { GPIb- } \\
\text { dependent activ. under shear }\end{array}$ \\
\hline 205 & Pld 2 & phospholipase D2 & MGI:892877 & A & 0 & 3 & 0 & 1 & no PLT phenotype \\
\hline 206 & $\mathrm{Plg}$ & plasminogen & MG1:97620 & A & 1 & 2 & 0 & 2 & $\downarrow$ fibrinolysis \\
\hline 207 & Plser1 & phospholipid scramblase 1 & MGI:893575 & A & & & 0 & 1 & normal PLT PS exposure \\
\hline 208 & PItp & phospholipid transfer protein & MGI:103151 & A & & & -1 & 1 & hyperlipidemia; EC dysfunction \\
\hline 209 & Pmel & premela no some protein (silver) & MGI:98301 & $\mathrm{A} / \mathrm{m}$ & & & 0 & 1 & mouse: abnormal pigmentation \\
\hline 210 & Podxl & podocalyxin & MGI:1351317 & P & -1 & 3 & -1 & 1 & unchanged PLT activ. \\
\hline 211 & Ppara & peroxisome proliferator-activated receptor al pha & MGI:104740 & A & & & -1 & 1 & no PLT phenotype \\
\hline 212 & Ppia & peptidyiprolyl isomerase A (cyclophilin A CypA] & MGI:97749 & A & -2 & 2 & 0 & 1 & $\downarrow$ PLT Ca fluxes \\
\hline 213 & Ppiff & $\begin{array}{l}\text { peptidyiprolyl isomerase } \mathrm{F} \text { Icyclophilin } \mathrm{F} \text {, mitochondrial cyclophilin } \\
\text { D) }\end{array}$ & MGI:214581 & A & 1 & 3 & 0 & 2 & $\begin{array}{l}\downarrow \text { PTL MPTP formation, } \downarrow \\
\text { procoagulant activity, } C R\end{array}$ \\
\hline 214 & Ppplce & $\begin{array}{l}\text { protein phosphatase 1, catalytic subunit, gamma isoform [Pp } 1 \mathrm{c} \\
\text { gamma) }\end{array}$ & MGI:104872 & A & -1 & 1 & 0 & 1 & $\downarrow$ PLT activ. with only thrombin \\
\hline 215 & Ppplrgb & protein phosphatase 1, regulatory subunit 98 (neurabin 2) & MGI:2387581 & A & -1 & 1 & 0 & 1 & $\begin{array}{l}\text { growth defect; } \downarrow \text { PLT aggr., Ca } \\
\text { with PAR peptide, TXA }\end{array}$ \\
\hline 216 & Prep & prolyicarboxypeptidase (angiotensinase C, PRCP) & MGI:1919711 & A & 2 & 2 & & & $\begin{array}{l}\text { antioxidant responsive } \\
\text { hypertension; } \uparrow \text { TG }\end{array}$ \\
\hline 217 & Prex1 & $\begin{array}{l}\text { phosphatidylinositol-3,4,5-trisphosphate-dependent Rac exchange } \\
\text { factor } 1 \text { (Prex1) }\end{array}$ & MGI:3040696 & A & & & -1 & 1 & $\downarrow$ PLT secr. and aggr. \\
\hline 218 & Prkaaz & $\begin{array}{l}\text { protein kinase, AMP-activated, alpha } 2 \text { catalytic subunit (AMPK } \\
\text { alpha2) }\end{array}$ & MGI:1336173 & A & -1 & 1 & 0 & 1 & $\downarrow \mathrm{CR}$ \\
\hline 219 & Prkca & protein kinase C, alpha (PKC alpha) & MGI:97601 & A & -2 & 3 & 0 & 1 & $\begin{array}{l}\downarrow \text { PLT activ. and secr. with } \\
\text { collagen }\end{array}$ \\
\hline 220 & Prkcb & protein kinase $\mathrm{C}$, beta (PKC beta) & MG1:97596 & A & -1 & 1 & & & $\begin{array}{l}\downarrow \text { PLT activ. with collagen, } \\
\text { fibrinogen }\end{array}$ \\
\hline 221 & Prked & protein kinase C, delta (PKC delta) & MG1:97598 & A & 1 & 2 & & & $\begin{array}{l}\downarrow \text { PLT activ. with thrombin; } \uparrow \\
\text { with collagen }\end{array}$ \\
\hline 222 & Prkce & protein kinase C, epsilon [PKC epsilon] & MG1:97599 & A & 1 & 2 & 1 & 2 & $\begin{array}{l}\uparrow \text { PLT activ. with ADP; no PLT } \\
\text { phenotype }\end{array}$ \\
\hline 223 & Prkca & protein kinase $\mathrm{C}$, theta (PKC theta) & MGI:97601 & A & 0 & 4 & 0 & 2 & $\begin{array}{l}\downarrow \text { PLT activ. with thrombin; } \uparrow \\
\text { with collagen (variable) }\end{array}$ \\
\hline 224 & Prkd2 & protein kinase D2 & MGI:2141917 & A & -1 & 1 & 0 & 1 & $\downarrow$ PLT dense granule secr. \\
\hline 225 & Prkg1 & protein kinase, cGMP-dependent, type I & MGI:108174 & A & & & -1 & 1 & $\begin{array}{l}\downarrow \text { PLT responses with VWF and } \\
\text { thrombin }\end{array}$ \\
\hline 226 & Proc & protein C & MGl:97771 & A & 2 & 2 & 1 & 2 & $\begin{array}{l}\text { spontaneous bleeding and } \\
\text { thrombosis }\end{array}$ \\
\hline 227 & Procr & protein C receptor, endothe lial (EPCR) & MGI:104595 & A & 1 & 1 & & & $\begin{array}{l}\text { embryonal fibrin formation, } \\
\text { lethality }\end{array}$ \\
\hline 228 & Pros1 & protein S, alpha & MGI:1095733 & $\overline{A E L}$ & 1 & 4 & & & $\begin{array}{l}\downarrow \text { Plasma protein S; thrombosis; } \\
\text { intercranial bleeding }\end{array}$ \\
\hline 229 & Proz & protein Z, vitamin K-dependent plasma glyco protein & MGI:1860498 & A & 2 & 3 & 0 & 1 & $\downarrow$ inhibition of $\mathrm{FXa}, \mathrm{FXla}$ \\
\hline 230 & Pten & phosphatase and tensin homolog (PTEN) & MGI:109583 & A & & & 1 & 1 & $\begin{array}{l}\uparrow \text { PLT count; } \uparrow \text { PLT act } v \text {. with } \\
\text { collagen }\end{array}$ \\
\hline
\end{tabular}




\begin{tabular}{|c|c|c|c|c|c|c|c|c|c|}
\hline 231 & Ptger3 & prostaglandin E receptor 3, subtype EP3 (EP3 receptor) & MGI:97795 & A & -1 & 2 & -1 & 1 & $\downarrow$ PLT act $v$. with PGE2 \\
\hline 232 & Ptgir & prostaglandin I receptor (IP receptor) & MG1:99535 & A & 2 & 1 & & & $\downarrow$ PLT responses with PGI2 \\
\hline 233 & Ptgs1 & prostaglandin-endoperoxide synthase 1 (cyclooxygenase 1, coxi) & MGI:97797 & A & 0 & 4 & -1 & 2 & $\begin{array}{l}\downarrow \text { EC PGI2 production; } \downarrow \text { PAl-1 in } \\
\text { SMC; } \downarrow \text { fibrinolysis }\end{array}$ \\
\hline 234 & Ptgs2 & prostaglandin-endoperoxide synthase 2 (cyclooxygenase $2, \operatorname{cox} 2$ ) & MG1:97798 & A & 0 & 3 & -1 & 1 & $\begin{array}{l}\downarrow \text { production PLT TXA, EC PGI2; } \\
\uparrow \text { TF expression }\end{array}$ \\
\hline 235 & Ptk2b & PTK2 prote in tyrosine kinase 2 beta (PYK2) & MGI:104908 & A & -1 & 3 & -1 & 1 & $\begin{array}{l}\downarrow \text { PLT integrin signaling; } \downarrow \text { activ. } \\
\text { with PAR4 peptide, TXA }\end{array}$ \\
\hline 236 & Ptpn1 & protein tyrosine phosphatase, non-receptor type 1(PTP1B) & MGI:97805 & A & -1 & 2 & & & no PLT phenotype \\
\hline 237 & Ptpn11 & protein tyrosine phosphatase, non-receptor type 11 (SHP2) & MGI:99511 & P & 0 & 2 & 0 & 1 & $\begin{array}{l}\text { macrothrombocytopenia, } \uparrow \text { PLT } \\
\text { activ. with fibrinogen }\end{array}$ \\
\hline 238 & Ptpn6 & protein tyrosine phosphatase, non-receptor type 6 (SHP1) & MGI:96055 & P & -1 & 2 & 0 & 1 & $\downarrow$ PLT act v. by GPVI \\
\hline 239 & Ptpr] & protein tyrosine phosphatase, receptor type, J (CO148) & MGI:104574 & A & -1 & 4 & -1 & 1 & $\begin{array}{l}\downarrow \text { PLT act } v \text {. with collagen, } \\
\text { fibrinogen ( } \downarrow \text { Src act } v \text {.) }\end{array}$ \\
\hline 240 & Rab27a & RAB27A, member RAS oncogene family (ashen) & MGI:1861441 & $\mathrm{N} / \mathrm{m}$ & & & -1 & 2 & PLT dense granule defect \\
\hline 241 & Rab27b & RAB27b, member RAS oncogene family & MGI:1931295 & A & & & -1 & 2 & $\begin{array}{l}\downarrow \text { PLT act } v \text {. with collagen, TXA, } \downarrow \\
\text { PLT granules }\end{array}$ \\
\hline 242 & Rabggta & Rab geranylgeranyl transferase, a subunit (gunmetal) & MGI:1860443 & $\mathrm{N} / \mathrm{m}$ & & & -1 & 1 & $\begin{array}{l}\text { macrothrombocytopenia; } \\
\text { combined PLT granule defect }\end{array}$ \\
\hline 243 & Rac1 & RAS-related C3 botulinum substrate 1 (Rac1) & MGI:97845 & A & -2 & 4 & -1 & 2 & $\begin{array}{l}\downarrow \text { PLT act } v \text {. with collagen } \\
\text { modocytin; } \downarrow \text { PLT spreading }\end{array}$ \\
\hline 244 & Rac2 & RAS-related C3 botulinum substrate $2(\operatorname{Rac} 2)$ & MGI:97846 & A & 0 & 1 & & & no PLT phenotype \\
\hline 245 & Ranbp10 & RAN binding prote in 10 (RanBP10) & MGI:1921584 & A & -1 & 2 & -1 & 2 & $\begin{array}{l}\downarrow \text { PLT shape change, altered } \\
\text { tubulin structure }\end{array}$ \\
\hline 246 & Rap1b & RAS related protein 16 (Rap $1 b$ ) & MGI:894315 & A & -2 & 1 & -1 & 1 & $\begin{array}{l}\downarrow \text { PLT aggr., integrin activ. (all } \\
\text { agonists) }\end{array}$ \\
\hline 247 & Rasgrp2 & RAS, guaryl releasing protein 2 (CalDAGGEF-1) & MGI:1333849 & A & -2 & 8 & -1 & 1 & $\downarrow$ PLT integrin activ. (all ago nists) \\
\hline 248 & Rgs2 & regulator of G-protein signaling 2 (RGS2) & MGI:1098271 & A & 0 & 2 & & & $\begin{array}{l}\text { unchanged PLT agg. (protein not } \\
\text { expressed in mouse PLTs) }\end{array}$ \\
\hline 249 & Rhoa & ras homo log gene family, member $A$ (AhoA) & MGI:1096342 & P & -2 & 2 & -1 & 1 & $\begin{array}{l}\text { macrothrombocytopenia, } \downarrow \text { PLT } \\
\text { activ. with ADP, thrombin }\end{array}$ \\
\hline 250 & Rhog & ras homolog gene family, member $\mathrm{G}$ (RhoG) & MGI:1928370 & A & -1 & 3 & 0 & 1 & $\begin{array}{l}\downarrow \text { PLT secr. and integrin activ. } \\
\text { with collagen }\end{array}$ \\
\hline 251 & Robol 1 & roundabout homolog 1 (Robol) & MGI:1274781 & B & -2 & 2 & -1 & 1 & $\begin{array}{l}\text { agonist impairs PLT adh., } \\
\text { spreading }\end{array}$ \\
\hline 252 & Scarb1 & scavenger receptor class B, member 1 [SRBI] & MGI:893578 & A & 0 & 2 & & & $\begin{array}{l}\downarrow \text { PLT act } v \text {. with ADP, thrombin, } \\
\text { collagen; } \downarrow \text { PLT cholesterol }\end{array}$ \\
\hline 253 & Selp & selectin, platelet (P-selectin, CD62P) & MGI:98280 & $A B$ & -1 & 5 & 0 & 3 & $\begin{array}{l}\downarrow P L T \text { (MP) interactions with } \\
\text { WBC }\end{array}$ \\
\hline 254 & Selplg & selectin, platelet p-selectin ligand (PSGL-1) & MGI:106689 & A & -2 & 2 & & & $\downarrow P L T$ (MP) interaction with WBC \\
\hline 255 & Sema4d & $\begin{array}{l}\text { sema domain, immunoglobulin domain, transmembrane doma in } \\
\text { and short cytoplasmic domain (semaphorin-40, CD100) }\end{array}$ & MGI:109244 & A & -2 & 3 & & & $\downarrow$ PLT act $v$. with collagen \\
\hline 256 & Serpina10 & protein Z dependent protease inhibitor (ZPI) & MG1:2667725 & A & 2 & 2 & & & $\downarrow$ inhibition of FXa, FXla \\
\hline 257 & Serpinc1 & $\begin{array}{l}\text { serine or cyste ine peptidase inhibitor, clade } \mathrm{C} \text {, member } 1 \\
\text { (antithrombin) }\end{array}$ & MGI:88095 & A & 1 & 1 & 0 & 1 & $\downarrow$ heparin effect on coagulation \\
\hline 258 & Serpind1 & $\begin{array}{l}\text { serine or cyste ine peptidase inhibitor, clade D, member } 1 \text { (heparin } \\
\text { cofactor II) }\end{array}$ & MG1:96051 & A & 1 & 2 & & & $\begin{array}{l}\downarrow \text { thrombin inhibition by } \\
\text { dermatan sulfate }\end{array}$ \\
\hline 259 & Serpine1 & $\begin{array}{l}\text { serine or cyste ine peptidase inhibitor, clade } \mathrm{E} \text {, member } 1 \\
\text { [plasminogen activator inhibitor } 1, \text { PAI-1] }\end{array}$ & MG1:97608 & A & -1 & 8 & 0 & 6 & $\uparrow$ fibrinolysis \\
\hline 260 & Serpine2 & $\begin{array}{l}\text { serine or cyste ine peptidase inhibitor, clade E, member } 2 \\
\text { [protease nexin 1, PN1] }\end{array}$ & MGI:101780 & A & 1 & 3 & & & $\begin{array}{l}\text { no PLT alpha granules; } \uparrow \text { tPA and } \\
\text { uPA; } \uparrow \text { fibrinolysis }\end{array}$ \\
\hline 261 & Serpinf2 & $\begin{array}{l}\text { serine or cysteine peptidase inhibitor, clade } F \text {, member } 2 \text { [aloha } 2 \\
\text { antiplasmin] }\end{array}$ & MGI:107173 & A & -1 & 2 & 0 & 4 & $\uparrow$ fibrinolysis \\
\hline 262 & 5gk1 & serum/glucocorticoid regulated kinase 1 (SGK1) & MGI:1340052 & A & -2 & 1 & 0 & 1 & PLTs with $\downarrow$ Orail expression \\
\hline 263 & 5 hab3 & SH2B adaptor protein 3 (Lnk) & MGI:893598 & $\mathrm{AH}$ & -1 & 3 & -1 & 1 & $\begin{array}{l}\downarrow \text { PLT spread., integrin act v., } \downarrow \\
\text { CR }\end{array}$ \\
\hline 264 & 5h2d1a & SH2 domain protein $1 \mathrm{~A}$ (SAP) & MGI:1328352 & A & -1 & 1 & 0 & 1 & no clear PLT phenotype \\
\hline 265 & Slamf1 & $\begin{array}{l}\text { signaling lymphocytic activation molecule family member } 1 \text { (SLAM, } \\
\text { CD150) }\end{array}$ & MGI:1351314 & A & -1 & 1 & 0 & 1 & $\begin{array}{l}\downarrow \text { PLT act v. with thrombin, } \\
\text { collagen }\end{array}$ \\
\hline 266 & 5lc30a4 & solute carrer family 30, member 4 (lethal milk) & $M G 1: 1345282$ & $\mathrm{~N} / \mathrm{m}$ & & & 0 & 1 & mouse: abnormal pigmentation \\
\hline 267 & 5lc45a2 & solute carrier family 45, member 2 (underwhite) & MGI:2153040 & $\mathrm{N} / \mathrm{m}$ & & & 0 & 1 & mouse: abnormal pigmentation \\
\hline 268 & $51 c 4 a 1$ & $\begin{array}{l}\text { solute carrier family } 4 \text { anion exchanger, member } 1 \text { (band } 3 \\
\text { protein) }\end{array}$ & MGI:109393 & A & 1 & 1 & & & $\begin{array}{l}\text { spherocytc aBCs; } \uparrow \mathrm{MP} \\
\text { shedding, PS exposure }\end{array}$ \\
\hline 269 & 5lc7a11 & solute carrier family 7 member-a11 (subtle gray) & MGI:1347355 & $\mathrm{N} / \mathrm{m}$ & & & -1 & 1 & mouse: abnormal pignentation \\
\hline 270 & Smpd1 1 & $\begin{array}{l}\text { sphingomyelin phosphodiesterase 1, acid lysosomal (acid } \\
\text { sphingomyelinase) }\end{array}$ & MGI:98325 & A & -1 & 1 & 0 & 1 & $\begin{array}{l}\downarrow \text { PLT act } v \text {. with thrombin, } \\
\text { collagen }\end{array}$ \\
\hline
\end{tabular}




\begin{tabular}{|c|c|c|c|c|c|c|c|c|c|}
\hline 271 & Sic & Rous sarcoma oncogene (Src kinase) & MGI:98397 & A & 0 & 1 & 0 & 1 & $\downarrow$ PLT spreading on fibrinogen \\
\hline 272 & Sif & serum response factor & MGI:106658 & $\mathrm{p}$ & & & -1 & 1 & $\begin{array}{l}\downarrow \text { MGK maturat on; altered PLT } \\
\text { cytoskeleton }\end{array}$ \\
\hline 273 & Sign & sergycin & MG1:97756 & A & -1 & 1 & & & $\downarrow$ PLT secr. and aggr. \\
\hline 274 & 5tat3 & signal transducer and activator of transcription 3 (Stat3) & MGI:103038 & P & -1 & 1 & & & $\begin{array}{l}\uparrow \text { PLT activ. with fibrinogen, } \uparrow \\
\mathrm{CR}\end{array}$ \\
\hline 275 & $\begin{array}{l}\text { Stat5a } \\
\text { (5tat5b) }\end{array}$ & $\begin{array}{l}\text { signal transducer and activator of transcription } 5 A+58 \text { [Stat5a + } \\
\text { Stat5b] }\end{array}$ & MGI:103036 & A & 1 & 1 & & & $\begin{array}{l}\text { double KO: } \downarrow \text { growth hormone } \\
\text { signaling: } \uparrow \text { fibrin }\end{array}$ \\
\hline 276 & $\begin{array}{l}\text { Stat5b } \\
\text { (5tat5a) }\end{array}$ & $\begin{array}{l}\text { signal transducer and activator of transcription 5B + 5A [Stat5b + } \\
\text { Stat5a) }\end{array}$ & MGI:103035 & A & 1 & 1 & & & $\begin{array}{l}\text { do uble KO: } \downarrow \text { growth hormone } \\
\text { signaling: } \uparrow \text { fibrin }\end{array}$ \\
\hline 277 & Stim1 & stromal interaction molecule 1 (STIM1) & MGI:107476 & $\mathrm{HP}^{\mathrm{P}}$ & -1 & 7 & -1 & 1 & $\begin{array}{l}\downarrow \text { PLT activ. with multiple } \\
\text { agonists }\end{array}$ \\
\hline 278 & Stim2 & stromal interaction molecule 2 (STIM2) & MGI:2151156 & A & 0 & 1 & 0 & 1 & no PLT phenotype \\
\hline 279 & 5vil & supervillin & MGI:2147319 & A & 1 & 1 & & & $\begin{array}{l}\text { langer PLTs, shorter closure time } \\
\text { (PFA-100) }\end{array}$ \\
\hline 280 & $5 y k$ & spleen tyrosine kinase (Syk) & MGI:99515 & $\mathrm{H}$ & & & 0 & 2 & $\downarrow$ PLT activ. with collagen, CLEC2 \\
\hline 281 & Tac1 & tachykinin 1 & MGI:98474 & A & -2 & 1 & & & not indicated \\
\hline 282 & Tacr1 & tachykinin (neurokinin) receptor-1 & MG1:98475 & A & -2 & 1 & -1 & 1 & $\downarrow$ PLT response to tachykinins \\
\hline 283 & Tbxa2r & thromboxane $A 2$ receptor (TP receptor) & MGI:98496 & $A B$ & -1 & 2 & -1 & 2 & $\downarrow$ PLT activ. with TXA \\
\hline 284 & Tbxas1 & thromboxane A synthase 1, platelet & MGI:98497 & A & & & -1 & 1 & $\downarrow$ PLT activ. with arachidonate \\
\hline 285 & Tfpi & tissue factor pathway inhibitor (Tfpi) & MGI:1095418 & AM & 1 & 2 & 0 & 2 & $\downarrow$ plasma TFPI; $\downarrow$ TF activ. \\
\hline 286 & Tgfb1 & transforming growth factor, beta 1 & MG1:98725 & A & & & -1 & 1 & $\downarrow$ PLT fibrinogen binding \\
\hline 287 & Tgfb1l1 & transforming growth factor beta 1 induced transcript 1 (Hic-5) & $M G 1: 102784$ & A & -1 & 1 & -1 & 1 & $\begin{array}{l}\downarrow \text { PLT integrin act v., } \downarrow \text { aggr. with } \\
\text { thrombin }\end{array}$ \\
\hline 288 & $\mathrm{Tgfbl}$ & transforming growth factor, beta induced (TGFBI) & MGI:99959 & A & -1 & 1 & & & mutation: $\uparrow$ expression in PLTs \\
\hline 289 & Thbod & thrombomodulin & MGI:98736 & $\overline{A E}$ & 2 & 2 & 0 & 1 & $\begin{array}{l}\text { mutation: } \uparrow \text { coagulat on } \backslash \downarrow \\
\text { protein } C \text { activ.) }\end{array}$ \\
\hline 290 & Thbs1 & thrombospondin 1 & MG1:98737 & A & 0 & 3 & -1 & 2 & $\begin{array}{l}\downarrow \text { TGF-beta activ.; } \uparrow \text { VWF } \\
\text { degradation }\end{array}$ \\
\hline 291 & Thbs2 & thrombospondin 2 & MG1:98738 & A & & & -1 & 1 & vesel wall dysfunctionality \\
\hline 292 & Tin1 & talin 1 & MGI:1099832 & AP & -2 & 4 & -1 & 2 & $\downarrow$ PLT aggr. and integrin activ. \\
\hline 293 & $\begin{array}{l}\text { Tnfrsf1a } \\
\text { (Tnfrsf1b) }\end{array}$ & $\begin{array}{l}\text { tumor necrosis factor receptor superfamily, member } 1 \mathrm{a}+1 \mathrm{~b} \text { (TNF- } \\
\text { R1) }\end{array}$ & $M G 1: 13148 B 4$ & A & 0 & 1 & & & $\downarrow$ PLT response with TNF-alpha \\
\hline 294 & $\begin{array}{l}\text { Tnfrsfib } \\
\text { (Tnfrsf1a) }\end{array}$ & $\begin{array}{l}\text { tumor necrosis factor receptor superfamily, member } 1 b+1 a \text { (TNF- } \\
\text { R2) }\end{array}$ & MGI:13148B3 & A & 0 & 1 & & & $\downarrow$ PLT response with TNF-alpha \\
\hline 295 & Tph1 & tryptophan hydroxylase 1 & MGl:98796 & A & -2 & 2 & -1 & 1 & $\downarrow$ PLT adh., secr. \\
\hline 296 & Treml1 & triggering receptor expressed on myeloid cells-like 1 (TLT1) & MGI:1918576 & A & & & -1 & 1 & $\downarrow$ fibrinogen binding, PLT aggr. \\
\hline 297 & Trp53 & transformation related protein 53 & MGI:98834 & A & & & -1 & 1 & $\downarrow$ fibrinogen binding, PLT secr. \\
\hline 298 & Trpe1 & $\begin{array}{l}\text { transient receptor potential cation channel, subfamily } \mathrm{C} \text {, member } \\
1(\mathrm{Trpc} 1)\end{array}$ & MGI:109528 & A & 0 & 2 & 0 & 1 & no PLT phenotype \\
\hline 299 & Trpe6 & $\begin{array}{l}\text { transient receptor potential cation channel, subfamily C, member } \\
6 \text { (Trpc6) }\end{array}$ & MGI:109523 & A & 0 & 4 & 0 & 2 & PLT function not described \\
\hline 300 & Tspan32 & tetraspanin 32 (TSSC6) & MGI:1350360 & A & -2 & 1 & -1 & 1 & $\begin{array}{l}\downarrow \text { PLT activ. with thrombin, } \\
\text { collagen; } \downarrow \mathrm{CR}\end{array}$ \\
\hline 301 & Tubb1 & tubulin, beta 1 class VI & MGI:107814 & A & & & -1 & 1 & $\begin{array}{l}\text { non-discoid PLTs, } \\
\text { thrombocytopenia }\end{array}$ \\
\hline 302 & Tyr & tyrosinase (acromelanic) & MG1:98880 & $\mathrm{A} / \mathrm{m}$ & & & 0 & 1 & $\begin{array}{l}\text { mouse: hypopigmentation, } \\
\text { albinism }\end{array}$ \\
\hline 303 & Tyro3 & TYRO3 protein tyrosine kinase 3 (Tyro3, Sky) & $M G 1: 104294$ & A & -2 & 2 & -1 & 2 & $\downarrow$ PLT aggr. with weak agonists \\
\hline 304 & Tyrp1 & tyrosinase-related prote in 1 (brown) & MG1:98881 & $\mathrm{A} / \mathrm{m}$ & & & 1 & 1 & mouse: hypopigmentation \\
\hline 305 & $\begin{array}{l}\text { Ubash3a } \\
\text { (Ubash 3b) }\end{array}$ & ubiquitin associated and $\mathrm{SH} 3$ do main containing, $\mathrm{A}+\mathrm{B}(\mathrm{TULA}+2)$ & MGI:1926074 & A & 2 & 1 & & & $\begin{array}{l}\text { do uble KO: } \uparrow \text { PLT actv. with } \\
\text { collagen (TULA not in PLTS) }\end{array}$ \\
\hline 306 & $\begin{array}{l}\text { Ubash3b } \\
\text { (Ubash 3a) }\end{array}$ & ubiquitin associated and $\mathrm{SH} 3$ do main conta ining, $\mathrm{B}+\mathrm{A}(\mathrm{TU} \cup \mathrm{LA} 2+1\rangle$ & MGI:1920078 & A & 2 & 1 & & & $\begin{array}{l}\text { do uble KO: } \uparrow \text { PLT actv. with } \\
\text { collagen (TULA not in PLTs) }\end{array}$ \\
\hline 307 & Unc13d & unc-13 homolog 0 (Munc13-4) & MGI:1917700 & A & -2 & 2 & -1 & 1 & † PLT dense granule secr. \\
\hline 308 & Vamp3 & vesicle-associated membrane protein 3 (Vamp3, cellubrevin) & MGI:1321389 & A & & & 0 & 1 & no PLT phenotype \\
\hline 309 & Vamp8 & vesicle-associated membrane protein 8 (endobrevin) & MGI:13368B2 & A & -1 & 1 & & & $\begin{array}{l}\downarrow \text { PLT dense and alpha granule } \\
\text { secr. }\end{array}$ \\
\hline 310 & Vav1 (Vav3) & vav1 +3 oncogene & MGI:98923 & A & -2 & 1 & & & $\begin{array}{l}\text { double KO: } \downarrow \text { PLT actv. and } \\
\text { spreading with collagen }\end{array}$ \\
\hline
\end{tabular}


Supplemental data Supplemental tables and figures chapter 8

\begin{tabular}{|c|c|c|c|c|c|c|c|c|c|}
\hline 311 & Vav3 (Vav1) & vav $3+1$ oncogene & MGI:1888518 & A & -2 & 1 & & & $\begin{array}{l}\text { double KO: } \downarrow \text { PLT activ. and } \\
\text { spreading with collagen }\end{array}$ \\
\hline 312 & Vcl & vinculin & MGI:98927 & P & 0 & 2 & -1 & 1 & $\begin{array}{l}\text { unchanged PLT activ. with } \\
\text { fibrinogen, collagen }\end{array}$ \\
\hline 313 & Vps33a & vacuolar protein sorting 33A (buff) & MGI:1924823 & $\mathrm{A} / \mathrm{m}$ & & & 0 & 1 & $\begin{array}{l}\text { mouse: hypopigmentation; no } \\
\text { PLT secr. defect }\end{array}$ \\
\hline 314 & Vtn & vitronectin & MGI:98940 & A & -1 & 6 & 0 & 1 & $\begin{array}{l}\text { 个 PLT activ. with thrombin; } \uparrow \\
\text { TG; stabilized PAI-1 }\end{array}$ \\
\hline 315 & Vwf & von Willebrand factor (Vwf) & MGI:98941 & $\mathrm{AL}$ & -2 & 14 & -1 & 7 & $\begin{array}{l}\downarrow \text { PLT adh. at high shear rate; } \downarrow \\
\text { TG }\end{array}$ \\
\hline
\end{tabular}


A

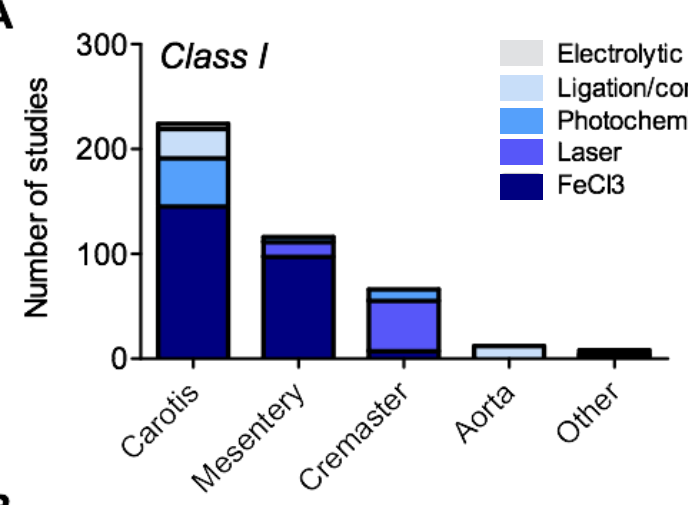

B

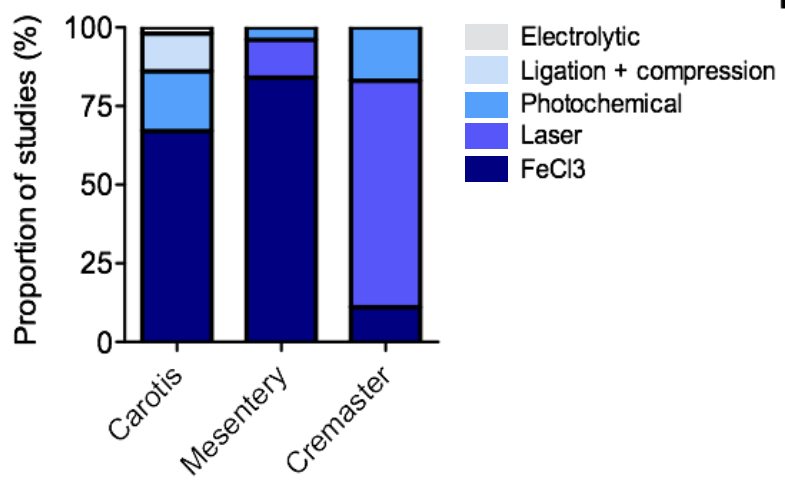

C
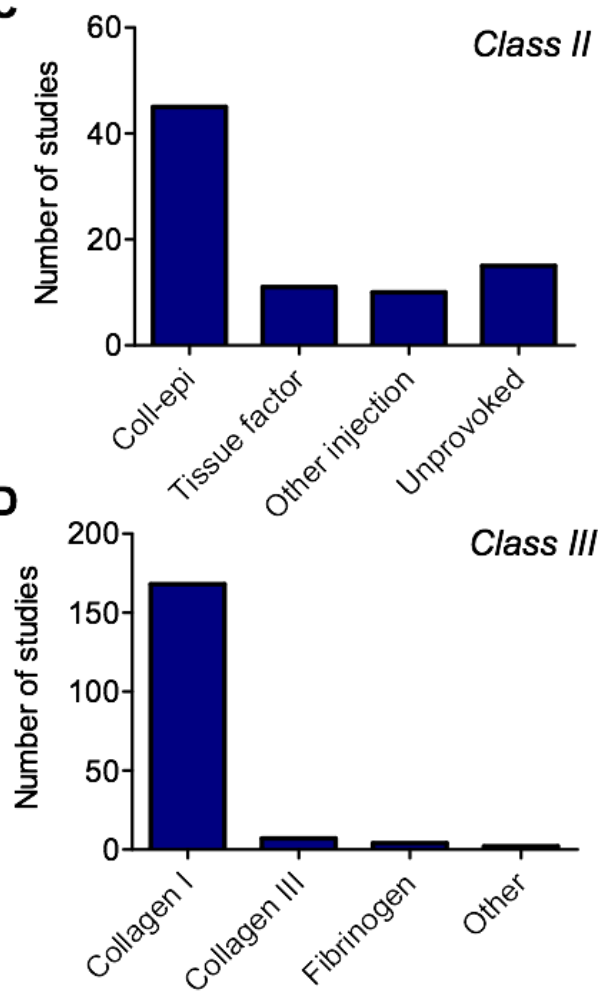

Supplemental Figure 1. Numbers of analyzed and scored studies in classes I-III with main experimental variables. (A, B) Class I studies with separation according to vascular bed or type of injury. (C) Class II studies with separation for type of systemic intervention. (D) Class III studies with separation for thrombogenic surface.

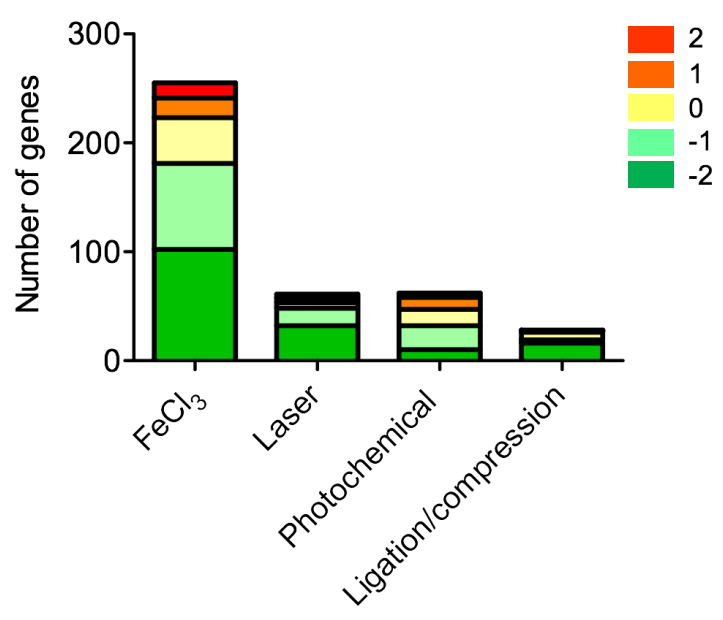

Supplemental Figure 2. Score distribution (5-point) of genes in class I, separated according to injury type $\left(\mathrm{FeCl}_{3^{\prime}}\right.$ laser, photochemical, ligation/compression). 


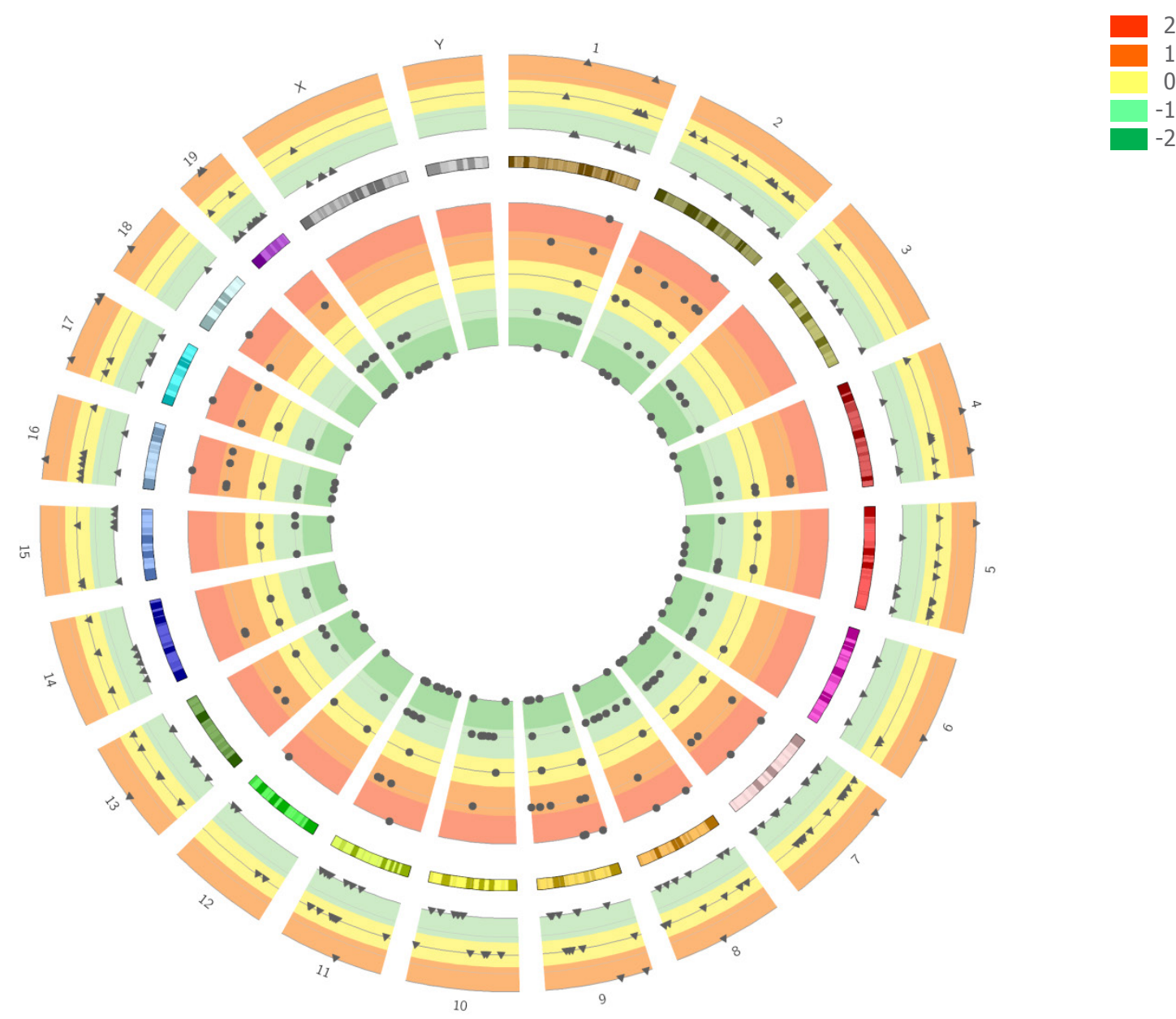

Supplemental Figure 3. Chromosomal location of mouse genes contributing to arterial thrombus formation and thromboembolism (classes I-III, inner ring, 5-point scores) or tail bleeding (class IV, outer ring, 3-point scores). 
Supplemental Figure 4. Over-representation analysis in Reactome for classes and study scores.

\begin{tabular}{|c|c|c|c|c|c|c|c|c|}
\hline \multirow{3}{*}{ No. } & \multirow{3}{*}{ Pathway name } & \multicolumn{3}{|c|}{ CLASS I-III,IV } & CLASS I-III & CLASS I-III & CLASS IV & CLASS IV \\
\hline & & Entities & Entities & Entities FDR & \multicolumn{4}{|c|}{ Entities FDR } \\
\hline & & found & total & score $-2,-1$ & score $-2,-1$ & score 0,1 & score $-2,-1$ & score 0,1 \\
\hline 1 & Formation of fibrin clot (Clotting cascade) & 16 & 34 & $1.94 \mathrm{E}-14$ & $1.91 \mathrm{E}-14$ & $6.97 \mathrm{E}-06$ & $4.24 \mathrm{E}-10$ & $1.26 \mathrm{E}-07$ \\
\hline 2 & GPVI-mediated activation cascade & 20 & 41 & $1.94 E-14$ & $1.91 \mathrm{E}-14$ & & $8.61 E-05$ & $6.85 \mathrm{E}-05$ \\
\hline 3 & Hemostasis & 86 & 536 & $1.94 E-14$ & $1.91 \mathrm{E}-14$ & $5.82 \mathrm{E}-14$ & $3.32 \mathrm{E}-14$ & 3.57E-14 \\
\hline 4 & Platelet activation, signaling and aggregation & 60 & 232 & $1.94 E-14$ & $1.91 E-14$ & $1.26 \mathrm{E}-10$ & 3.32E-14 & 3.57E-14 \\
\hline 5 & Response to elevated platelet cytosolic $\mathrm{Ca} 2+$ & 21 & 95 & $3.11 E-14$ & $4.81 E-12$ & & 7.52E-07 & $8.28 \mathrm{E}-08$ \\
\hline 6 & Cell surface interactions at the vascular wall & 21 & 108 & $2.47 \mathrm{E}-13$ & $5.67 \mathrm{E}-13$ & $6.55 \mathrm{E}-05$ & 4.27E-03 & $1.50 \mathrm{E}-12$ \\
\hline 7 & Platelet degranulation & 19 & 88 & $7.88 \mathrm{E}-13$ & $1.62 \mathrm{E}-10$ & 4.96E-03 & 3.65E-07 & 2.74E- 07 \\
\hline 8 & Platelet aggregation (Plug formation) & 15 & 49 & $3.82 \mathrm{E}-12$ & $1.23 \mathrm{E}-11$ & & $6.52 \mathrm{E}-11$ & \\
\hline 9 & Intrinsic coagulation pathway & 11 & 18 & $6.02 \mathrm{E}-12$ & 1.66E-11 & & 2.95E-08 & $2.89 \mathrm{E}-03$ \\
\hline 10 & CD28 co-stimulation & 11 & 37 & $9.16 \mathrm{E}-09$ & 1.66E-06 & & $3.00 \mathrm{E}-02$ & \\
\hline 11 & Integrin alphallb beta 3 signaling & 11 & 37 & $9.16 \mathrm{E}-09$ & $3.28 \mathrm{E}-08$ & & $2.88 \mathrm{E}-07$ & \\
\hline 12 & Platelet adhesion to exposed collagen & 8 & 16 & $5.28 \mathrm{E}-08$ & $1.02 \mathrm{E}-07$ & & 2.71E-03 & $6.30 \mathrm{E}-06$ \\
\hline 13 & Cell-cell communication & 15 & 143 & 4.30E-06 & $1.55 \mathrm{E}-05$ & & $5.21 E-03$ & $3.21 \mathrm{E}-03$ \\
\hline 14 & Syndecan interactions & 8 & 29 & 4.30E-06 & 7.14E-05 & & & $1.26 \mathrm{E}-04$ \\
\hline 15 & Grb2:Sos provides linkage to MAPK signaling for integrins & 7 & 20 & 5.07E-06 & 2.16E-06 & & $9.26 \mathrm{E}-07$ & \\
\hline 16 & Costimulation by the $\mathrm{CD} 28$ family & 12 & 94 & $1.00 \mathrm{E}-05$ & & & & \\
\hline 17 & Signaling by SCF-Kit & 15 & 155 & $1.00 \mathrm{E}-05$ & $7.72 \mathrm{E}-05$ & $3.42 \mathrm{E}-03$ & & $1.47 \mathrm{E}-03$ \\
\hline 18 & G-protein beta:gamma signaling & 8 & 34 & $1.07 \mathrm{E}-05$ & $7.72 E-05$ & & & \\
\hline 19 & DAP12 signaling & 16 & 181 & $1.11 E-05$ & $7.14 E-05$ & & & \\
\hline 20 & G alpha $(12 / 13)$ signaling events & 11 & 80 & $1.20 \mathrm{E}-05$ & 1.33E-04 & & & \\
\hline 21 & Transport of gamma-carboxylated protein precursors & 5 & 8 & $1.50 E-05$ & 3.84E-05 & $2.77 \mathrm{E}-03$ & 5.37E-03 & 4.19E-03 \\
\hline 22 & Extracellular matrix organization & 20 & 291 & 1.67E-05 & 1.27E-04 & & $1.58 \mathrm{E}-02$ & $1.14 \mathrm{E}-03$ \\
\hline 23 & Integrin cell surface interactions & 11 & 86 & 2.17E-05 & 2.64E-04 & & & 8.53E-04 \\
\hline 24 & Removal of propeptides from gamma-carboxylated proteins & 5 & 9 & $2.40 E-05$ & $5.71 E-05$ & $3.42 \mathrm{E}-03$ & $6.99 \mathrm{E}-03$ & $5.66 \mathrm{E}-03$ \\
\hline 25 & DAP12 interactions & 16 & 198 & $2.81 E-05$ & $1.57 \mathrm{E}-04$ & & & \\
\hline 26 & Sema4D induced cell migration and growth-cone collapse & 7 & 28 & $2.88 \mathrm{E}-05$ & 2.53E-04 & & 1.49E-02 & \\
\hline 27 & Semaphorin interactions & 10 & 72 & 2.90E-05 & 2.53E-04 & & 4.85E-02 & \\
\hline 28 & GPIb-IX-V activation signaling & 5 & 10 & 3.41E-05 & $7.72 E-05$ & & 4.94E-04 & \\
\hline 29 & Immune system & 52 & 1,507 & 7.19E-05 & & & & \\
\hline 30 & Sema $4 D$ in semaphorin signaling & 7 & 33 & $7.19 \mathrm{E}-05$ & 4.11E-04 & & $2.23 E-02$ & \\
\hline 31 & p130Cas linkage to MAPK signaling for integrins & 6 & 22 & $8.36 \mathrm{E}-05$ & 3.84E-05 & & 3.62E-05 & \\
\hline 32 & Platelet homeostasis & 11 & 103 & 8.63E-05 & 1.70E-04 & $5.44 E-05$ & 4.67E-02 & $6.59 E-05$ \\
\hline 33 & Thrombin signaling proteinase-activated receptors (PARs) & 7 & 35 & $9.36 \mathrm{E}-05$ & $1.40 \mathrm{E}-04$ & & 4.27E-03 & \\
\hline 34 & Extrinsic coagulation pathway & 4 & 6 & $1.02 \mathrm{E}-04$ & 1.12E-04 & & 4.67E-02 & \\
\hline 35 & Gastrin-CREB signaling pathway via PKC and MAPK & 17 & 256 & $1.20 \mathrm{E}-04$ & $8.05 E-05$ & & 4.67E-02 & \\
\hline 36 & Regulation of signaling by $\mathrm{Cbl}$ & 6 & 24 & $1.20 \mathrm{E}-04$ & $1.70 \mathrm{E}-04$ & & & \\
\hline 37 & Signal amplification & 7 & 37 & $1.20 \mathrm{E}-04$ & $1.40 \mathrm{E}-04$ & & $5.26 \mathrm{E}-04$ & \\
\hline 38 & Gamma-carboxylation of protein precursors & 5 & 14 & $1.28 \mathrm{E}-04$ & $5.71 E-05$ & & $1.98 \mathrm{E}-02$ & \\
\hline 39 & CD28 dependent PI3K/Akt signaling & 6 & 25 & $1.38 \mathrm{E}-04$ & & & & \\
\hline 40 & Interleukin-3, 5 and GM-CSF signaling & 8 & 55 & $1.55 \mathrm{E}-04$ & 2.64E-04 & & & $2.89 \mathrm{E}-03$ \\
\hline 41 & Common pathway & 5 & 16 & $2.13 E-04$ & $2.53 E-04$ & 9.14E-04 & 1.37E-04 & 2.10E-03 \\
\hline 42 & Netrin-1 signaling & 7 & 50 & $2.47 \mathrm{E}-04$ & & 5.44E-05 & & $1.90 \mathrm{E}-03$ \\
\hline 43 & Gamma-carboxylation, transport, and cleavage of proteins & 5 & 17 & $2.76 \mathrm{E}-04$ & $1.12 \mathrm{E}-04$ & & $3.00 \mathrm{E}-02$ & \\
\hline 44 & Non-integrin membrane-ECMinteractions & 8 & 61 & 3.03E-04 & & & & \\
\hline 45 & Regulation of Kit signaling & 5 & 18 & $3.48 \mathrm{E}-04$ & 4.11E-04 & & & 1.13E-05 \\
\hline 46 & Adaptive immune system & 35 & 919 & $3.94 \mathrm{E}-04$ & & & & \\
\hline 47 & Fc epsilon receptor (FCERI) signaling & 19 & 352 & 4.33E-04 & & & & \\
\hline 48 & Synthesis of PIPs at the plasma membrane & 7 & 48 & 4.41E-04 & $1.61 E-04$ & & & \\
\hline 49 & G alpha $(q)$ signaling events & 15 & 235 & 4.47E-04 & $2.53 \mathrm{E}-04$ & & & \\
\hline 50 & Constitutive PI3K/AKT signaling in cancer & 10 & 113 & $6.88 \mathrm{E}-04$ & & & & \\
\hline 51 & PI3K/AKT activation & 10 & 117 & $8.38 \mathrm{E}-04$ & & & & \\
\hline 52 & CTLA4 inhibitory signaling & 5 & 26 & $1.39 \mathrm{E}-03$ & & $3.42 \mathrm{E}-03$ & & $1.08 \mathrm{E}-03$ \\
\hline 53 & GPCR downstream signaling & 37 & 1,069 & $1.39 \mathrm{E}-03$ & & & & \\
\hline 54 & Signal transduction & 62 & 2,175 & $1.39 \mathrm{E}-03$ & & & & \\
\hline 55 & PECAM1 interactions & 4 & 14 & $1.49 \mathrm{E}-03$ & $1.72 \mathrm{E}-03$ & $6.06 \mathrm{E}-04$ & & $3.25 \mathrm{E}-06$ \\
\hline 56 & AKT-mediated inactivation of FOXO1A & 3 & 6 & $2.08 E-03$ & & & & \\
\hline 57 & CD28 dependent Vav1 pathway & 4 & 16 & $2.18 \mathrm{E}-03$ & & & 2.59E-02 & \\
\hline 58 & Signaling by GPCR & 39 & 1,201 & $2.18 \mathrm{E}-03$ & & & & \\
\hline 59 & Downstream signal transduction & 11 & 178 & 3.79E-03 & & & & \\
\hline 60 & Signaling by PDGF & 12 & 209 & $4.06 \mathrm{E}-03$ & & $5.44 E-05$ & & \\
\hline 61 & Dissolution of fibrin clot & (4) & (11) & & & $2.64 E-04$ & & $9.09 \mathrm{E}-07$ \\
\hline
\end{tabular}




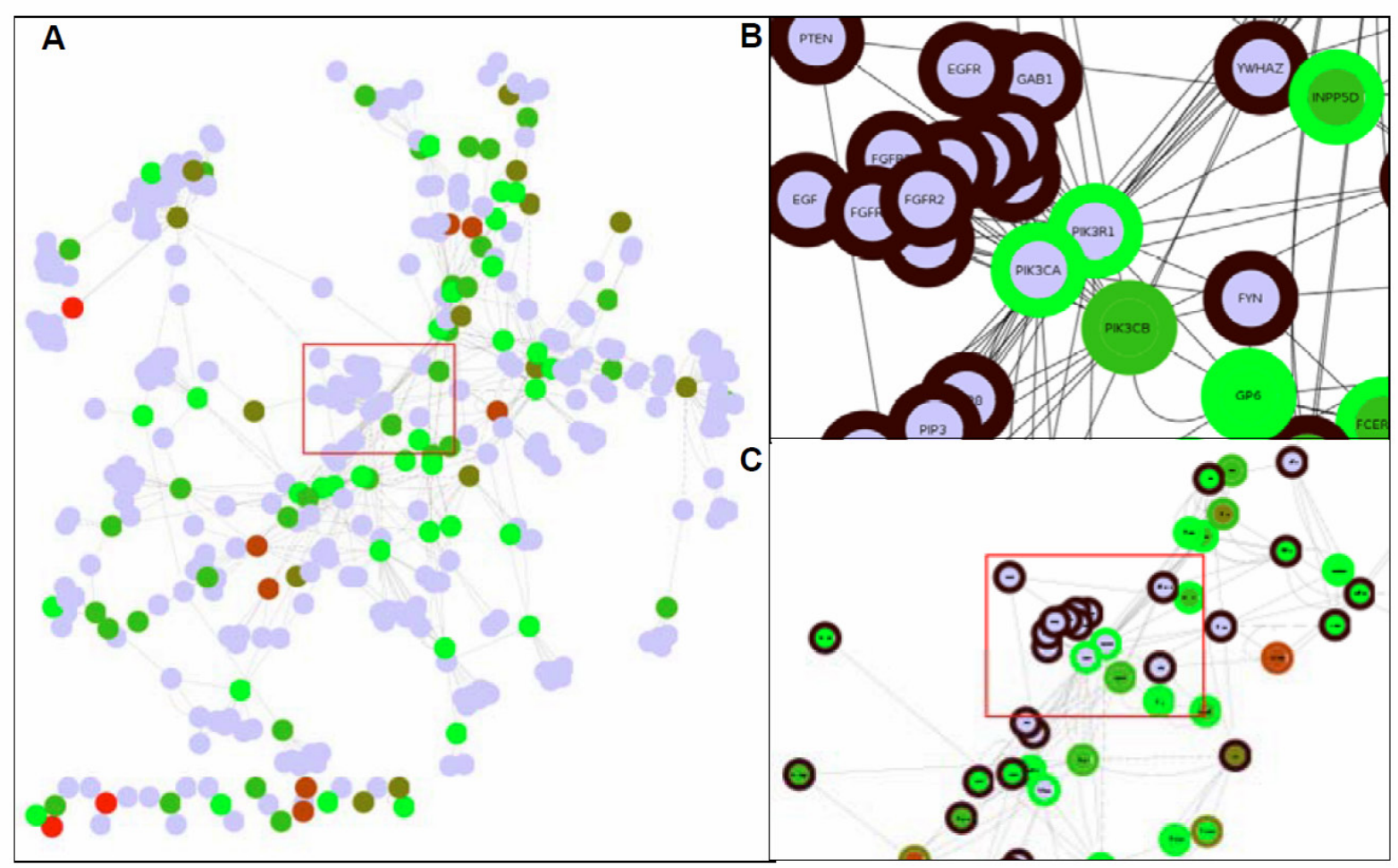

Supplemental Figure 5. Network visualization and analysis of genes in thrombus formation. Protein-protein interactions from the Reactome database were used to build a network of interacting proteins in Cytoscape. (A) Network with visualized five-point scores (green is negative score, red is positive score). (B, C) Magnification of indicated parts. Gray nodes display novel genes (proteins) not in database. Note that the gene FYN has multiple edges to genes with known influences on thrombus formation (GP6, PIK3CA, FCGER). It thus may have a high probability of involvement in thrombus formation. 